\title{
Myeloid corticoid receptors in CNS autoimmunity: Old targets for novel therapies
}

\author{
Dissertation \\ In partial fulfillment of the requirements for the degree \\ "Doctor rerum naturalium (Dr. rer. nat.)" \\ of the Georg-August University Göttingen \\ within the "Molecular Medicine" Study Program \\ at the Georg-August University School of Science (GAUSS) \\ submitted by \\ Elena Victoria Montes Cobos \\ born in \\ Badajoz, Spain
}

Göttingen, April 2016 


\section{THESIS COMMITTEE}

Prof. Dr. Holger M. Reichardt

(First referee)

Institute for Cellular and Molecular Immunology

University Medical Center, Göttingen

Prof. Dr. Lutz Walter

(Second referee)

Dep. of Primate Genetics

German Primate Center, Göttingen

PD Dr. Fred Lühder

Dep. of Neuroimmunology

Institute for Multiple Sclerosis Research and the Hertie Fondation

University Medical Center, Göttingen

\section{ADDITIONAL MEMBERS OF THE EXAMINATION BOARD}

\section{Prof. Dr. Christine Stadelmann-Nessler}

Institute for Neuropathology

University Medical Center, Göttingen

Prof. Dr. mult. Thomas Meyer

Lab. for Molecular Psychocardiology

University Medical Center, Göttingen

\section{Prof. Dr. Hubertus Jarry}

Dep. of Clinical and Experimental Endocrinology

University Medical Center, Göttingen

Date of the oral examination: $15^{\text {th }}$ June 2016 


\section{AFFIDAVIT}

I hereby declare that I have written this PhD thesis entitled "Myeloid corticoid receptors in CNS autoimmunity: Old targets for novel therapies" independently and with no other sources and aids than quoted. This thesis has not been submitted elsewhere for any academic degree.

Elena Victoria Montes Cobos

April 2016

Göttingen, Germany 


\section{TABLE OF CONTENTS}

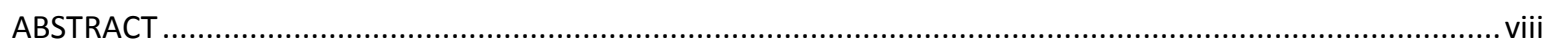

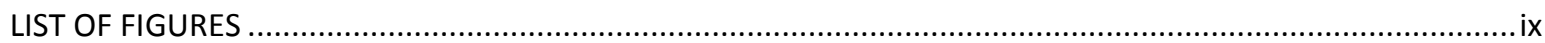

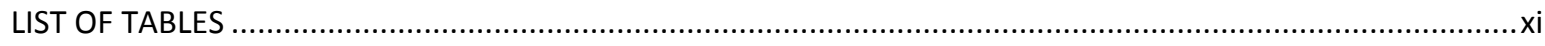

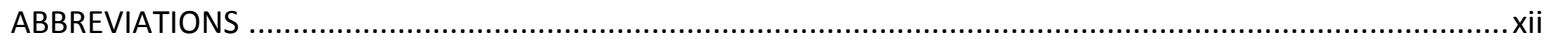

$1 \quad$ INTRODUCTION $\ldots \ldots \ldots \ldots \ldots \ldots \ldots \ldots \ldots$

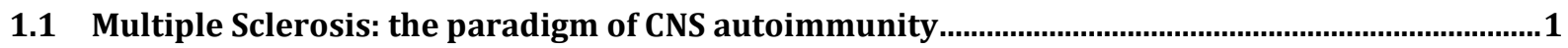

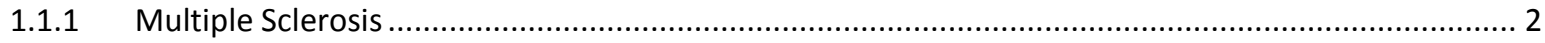

1.1.2 Animal models of MS - Experimental autoimmune encephalomyelitis ...................................... 3

1.2 The cellular players of CNS autoimmune inflammation ...............................................................

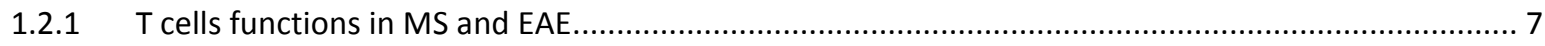

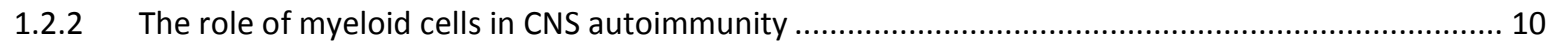

1.3 Immunomodulatory therapies for MS: glucocorticoids .................................................................. 14

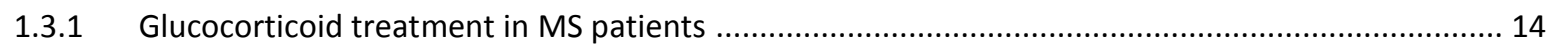

1.3.2 Molecular and cellular basis of GCs immunosuppression ................................................... 16

1.3.3 Mineralocorticoid receptor-mediated activities of GCs.......................................................... 17

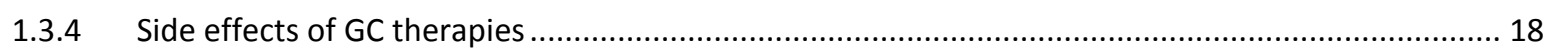

1.3.5 Emerging GC therapies: from dissociating ligands to nanoparticles....................................... 20

1.4 Objectives.

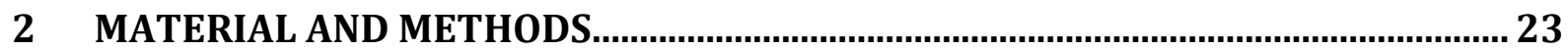

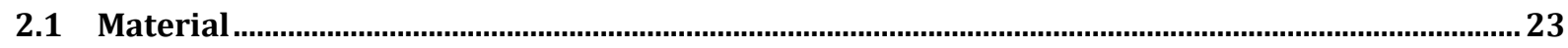

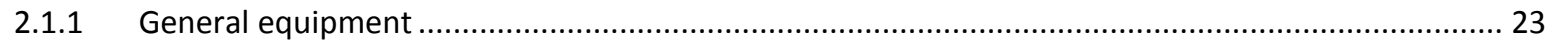

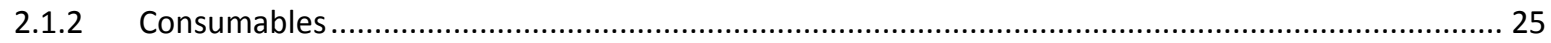

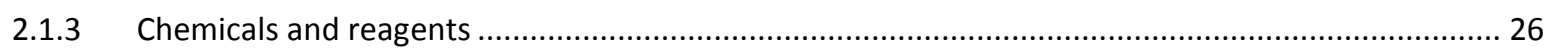

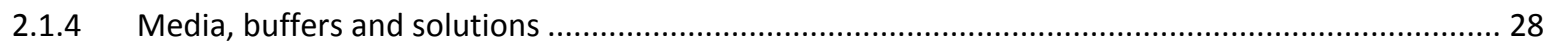

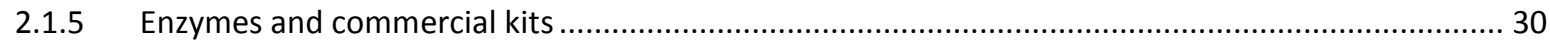

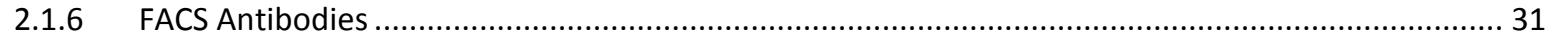

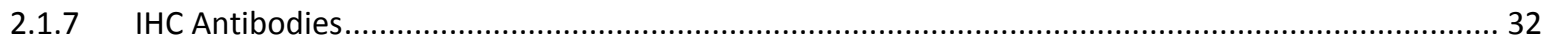




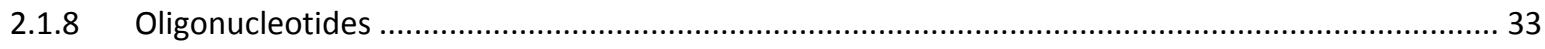

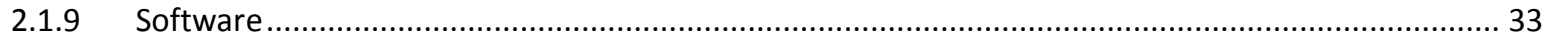

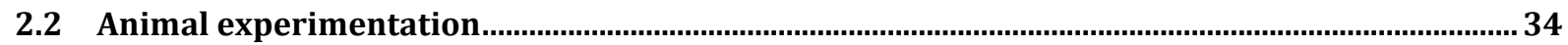

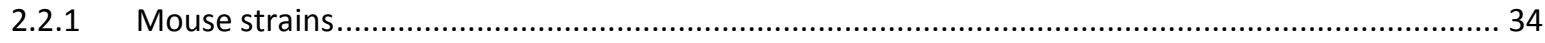

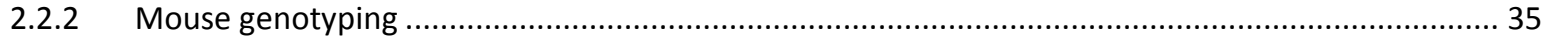

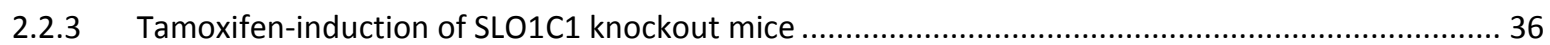

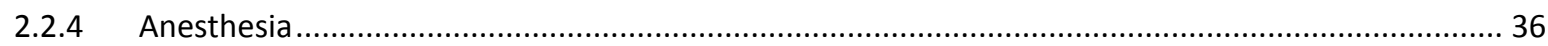

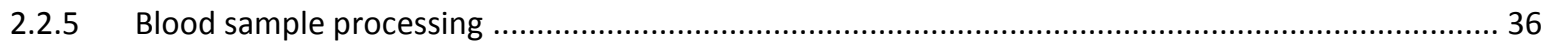

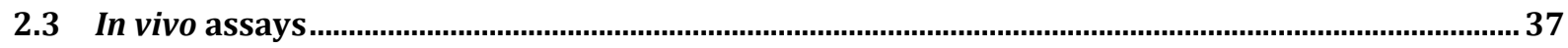

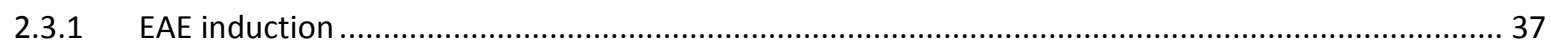

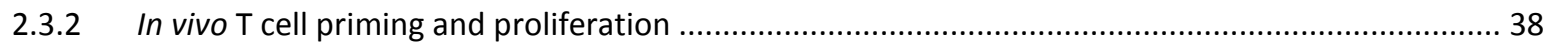

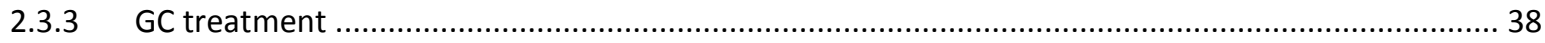

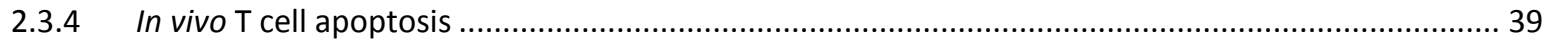

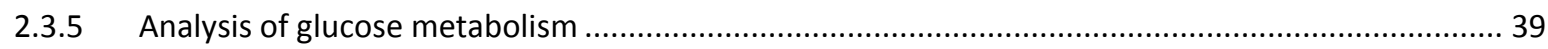

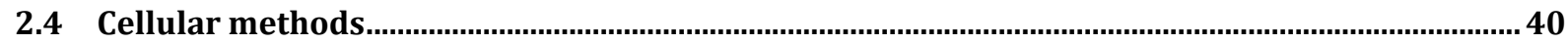

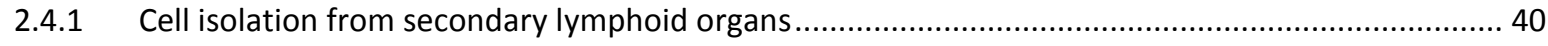

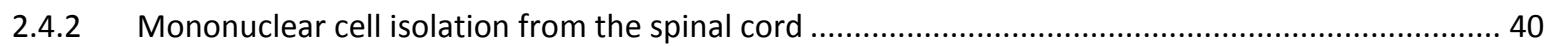

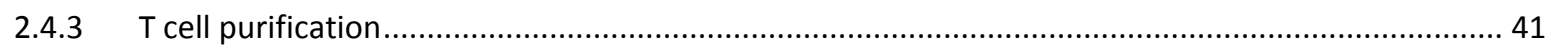

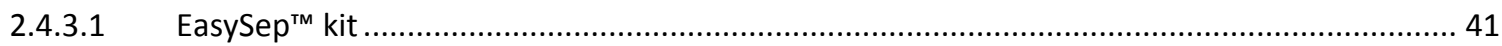

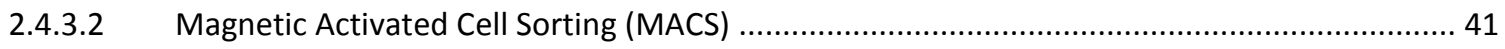

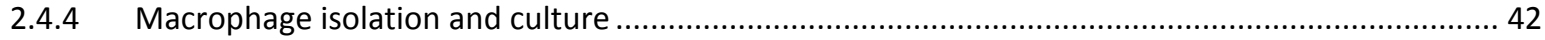

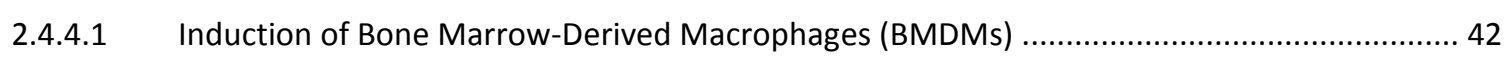

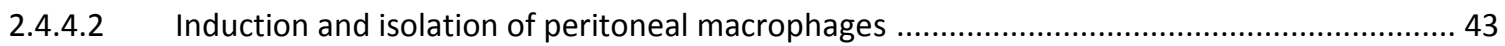

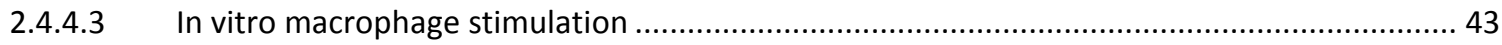

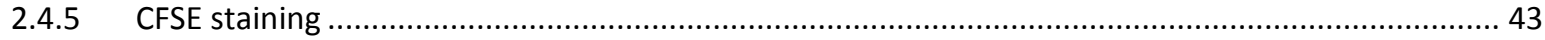

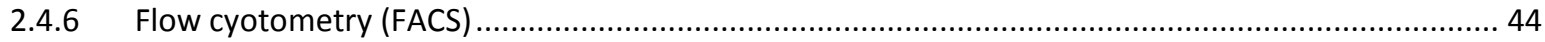

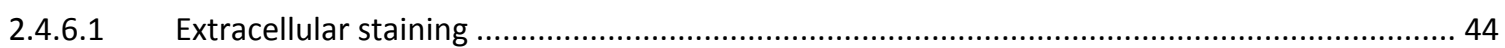

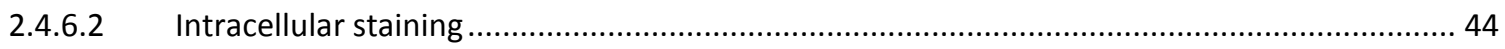

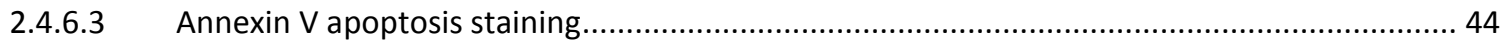

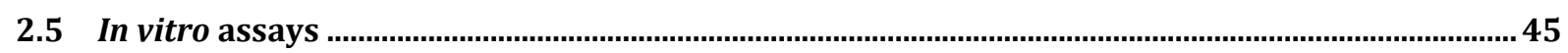

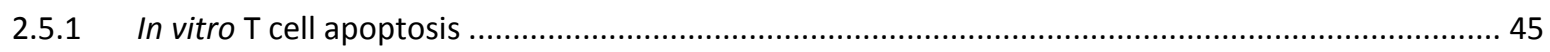

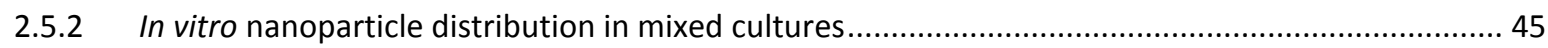

2.5.3 Ex vivo re-stimulation of MOG-specific effector T cells .......................................................45

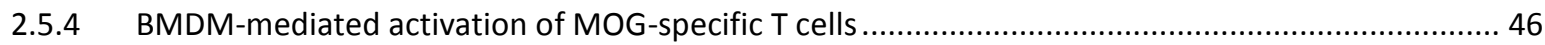




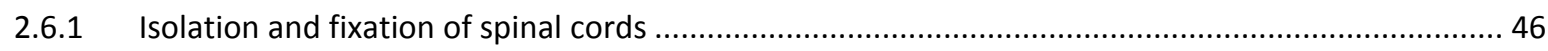

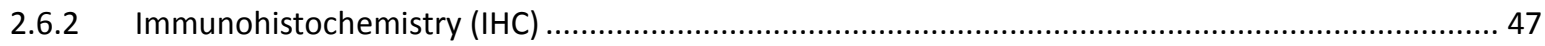

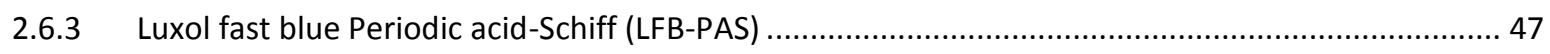

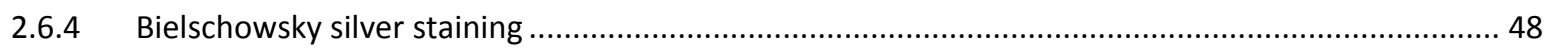

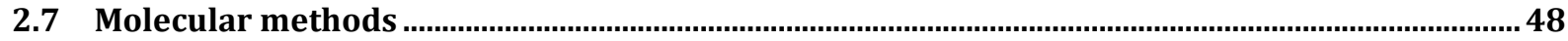

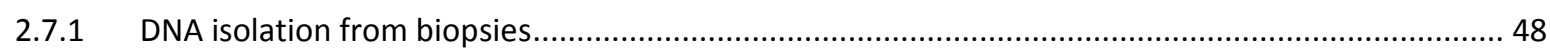

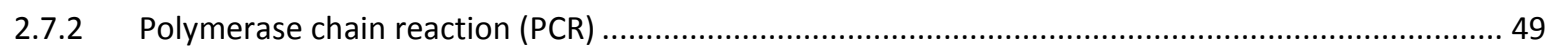

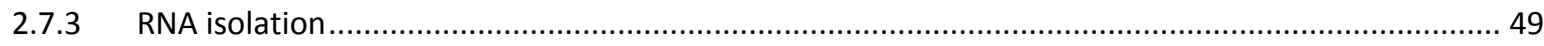

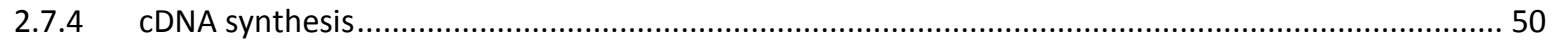

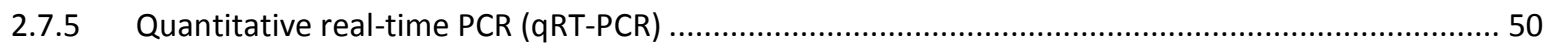

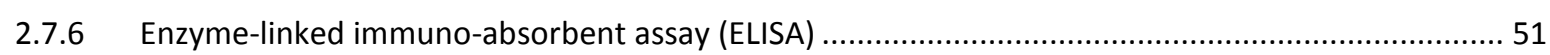

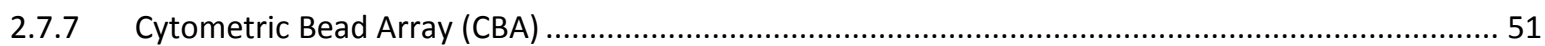

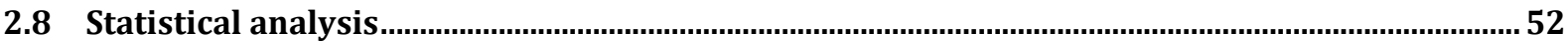

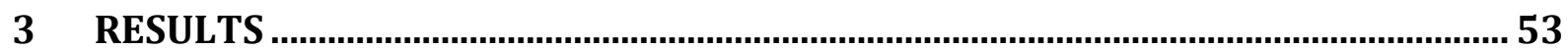

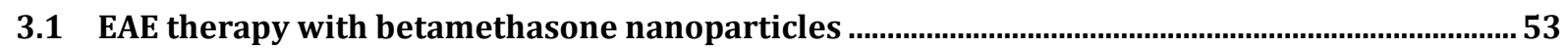

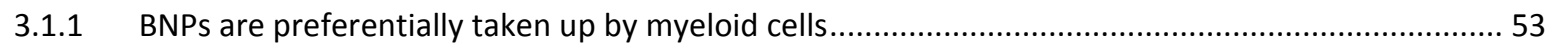

3.1.2 BNPs are more potent in modulating macrophages than T cell activity in vivo ..............................55

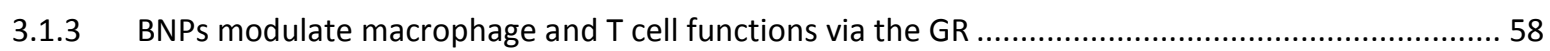

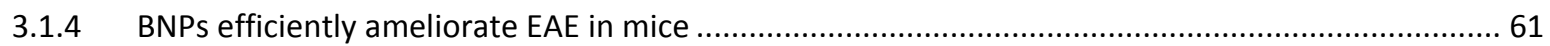

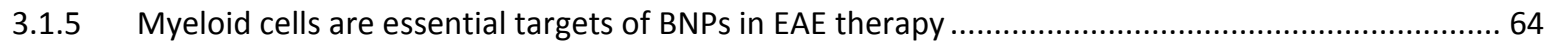

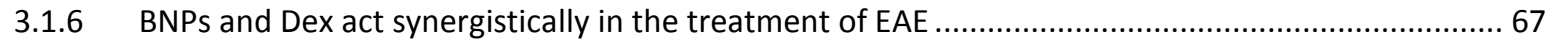

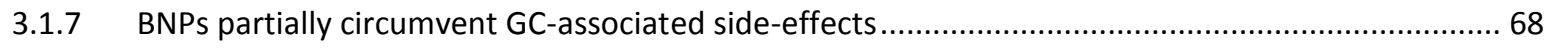

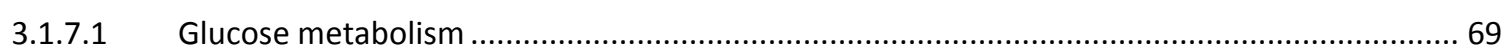

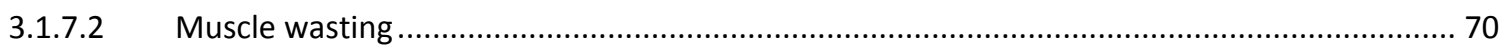

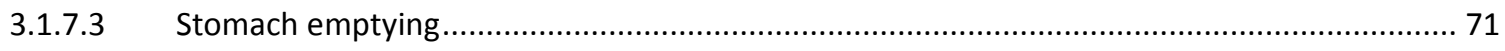

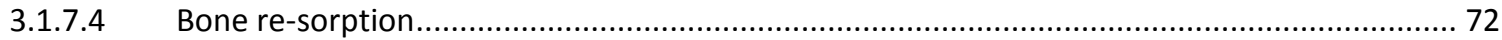

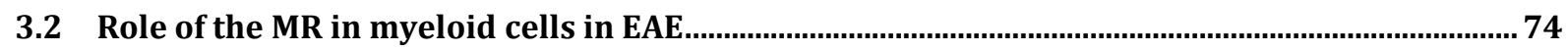

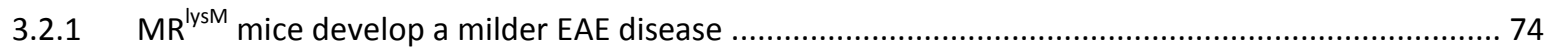

3.2.2 MR deletion alters the activation state of circulating and CNS-infiltrating monocytes................... 79

3.2.3 Monocytes and neutrophils in secondary lymphoid organs are not affected by the MR deletion ... 81

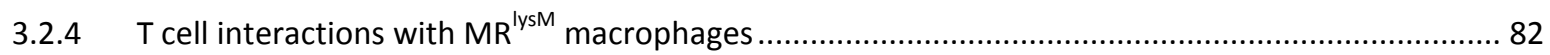

3.2.4.1 The cytokine secretion profile is altered in MR-deficient BMDMs.................................... 83

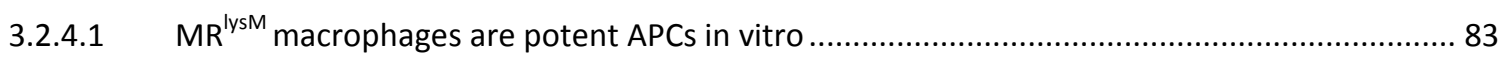

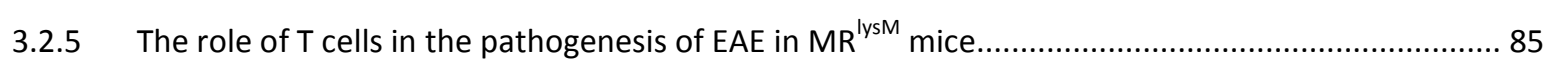

3.2.5.1 MR-deficiency does not impair MOG-specific T cell priming in vivo .................................... 85 
3.2.5.2 Secretion of Th1 and Th17 cytokines during EAE is impaired in MR ${ }^{\mathrm{lysM}}$ mice .......................86

3.2.5.3 MR deficiency in myeloid cells leads to increased numbers of peripheral Treg cells .............. 87

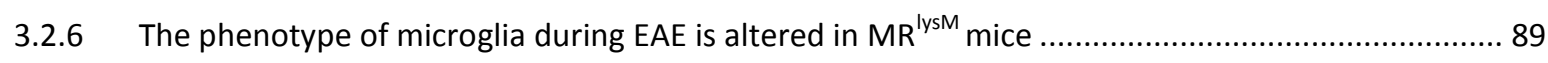

$4 \quad$ DISCUSSION

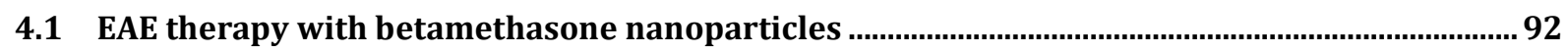

4.1.1 BNPs are anti-inflammatory in different immune cell types, albeit with different efficacy ............. 93

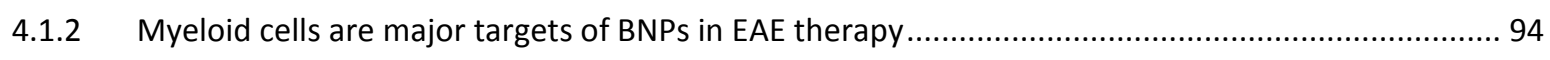

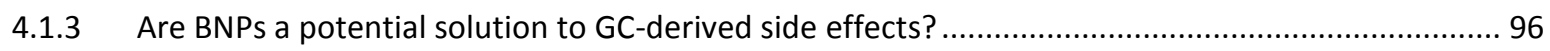

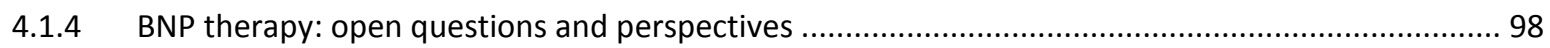

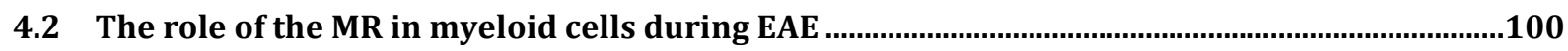

4.2.1 MR deficiency in myeloid cells promotes M2-like polarization and causes a milder EAE

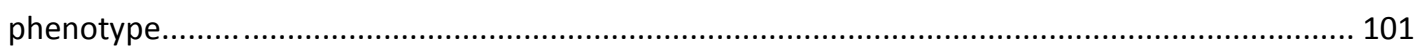

4.2.2 MR deletion in myeloid cells alters T cell responses during EAE ........................................... 102

4.2.3 MR deletion in myeloid cells partially restores peripheral tolerance ......................................... 105

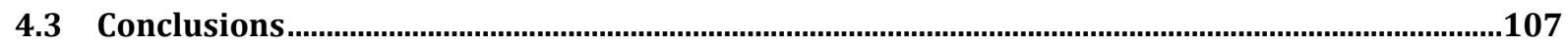

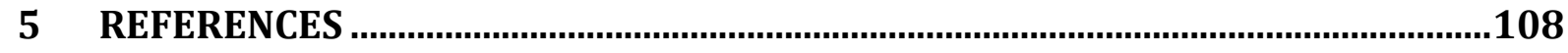

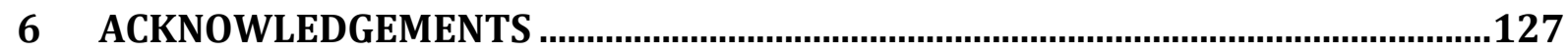




\section{ABSTRACT}

Since the 1950s, glucocorticoids (GCs) have been the most widely employed drugs in the treatment of inflammatory and autoimmune disorders, such as multiple sclerosis (MS). Short-time application of high-dose GCs is the first line therapy for acute relapses of MS. Several clinical studies even suggest that prolonged GC pulsed therapy may slow down MS disease progression as well. However, a plethora of GC-associated side effects derived from the ubiquitous expression of the glucocorticoid receptor (GR) restricts the use of these drugs. Therefore, to assess the full potential of GCs new pharmacological formulations and more insights about their mechanisms of action are needed.

For many years, T cells were proposed to be major targets of GCs in the treatment of neuroinflammation. In contrast, the relevance of myeloid cells for GC therapy is highlighted in this doctoral thesis. Using experimental autoimmune encephalomyelitis (EAE) as a mouse model of CNS autoimmunity, we directed GC therapy to the myeloid compartment by means of state-of-the-art inorganic-organic hybrid nanoparticles (IOH-NPs). Although IOH-NPs loaded with betamethasone (BNPs) modulated both T cell survival and macrophage activation in vitro, in vivo BNPs selectively targeted myeloid cells. Moreover, BNPs achieved a therapeutic efficacy comparable to free GCs in the treatment of EAE. In this study BNPs were also proposed as an alternative to circumvent GCderived side effects, however our data did not provide conclusive information in this respect yet. Additionally, the roles of the two different GC-responsive nuclear receptors, the GR and the mineralocorticoid receptor (MR), were investigated in myeloid cells. In this cell compartment, GCs can act either via the GR in a deactivating manner, or via the MR promoting a pro-inflammatory state. Hence, we hypothesized that altering the balance between the GR and the MR might influence the course of EAE. Indeed, mice selectively devoid of MR in myeloid cells developed a milder EAE disease course compared to their littermate controls, and presented with a lower degree of demyelination. Consistent with these results, monocytes/macrophages exhibited a M2 phenotype in both the CNS and the periphery, and the proportion of reactive microglia in the spinal cord was reduced as well. Furthermore, our experiments revealed that, in consequence of the general myeloid anti-inflammatory state, Treg cell numbers increased in secondary lymphoid organs, where cytokine release by effector T cells was consequently impaired.

Taken together, this study provides evidence of the important implications of myeloid cells in the response to GCs during autoimmune inflammation, and supports the targeted delivery to the myeloid compartment as an alternative to improve the therapeutic features of synthetic GCs. 


\section{LIST OF FIGURES}

Figure 1.1 | Immune cells participating in MS and EAE pathology. ............................................. 6

Figure 1.2 | Macrophage polarization induced by different stimuli. …............................................. 12

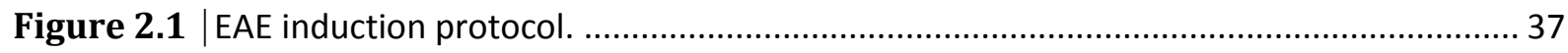

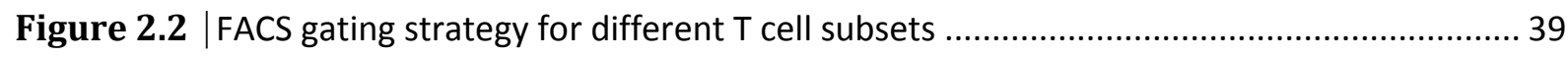

Figure 3.1 | BNPs are efficiently but differentially taken up by immune cells in vitro........................ 54

Figure 3.2 | BNPs exert potent immunosuppressive activity on peritoneal macrophages in vivo..... 56

Figure 3.3 | BNPs do not efficiently induce T cell apoptosis in vivo................................................. 58

Figure 3.4 | BNPs do not reduce MHC class II expression on $\mathrm{GR}^{\mathrm{lysM}}$ macrophages in vivo. ................59

Figure 3.5 |BNPs depend on the presence of the GR for the induction of T cell apoptosis...............60

Figure 3.6 | The therapeutic efficacy of BNPs in the treatment of EAE is similar to free GCs........... 62

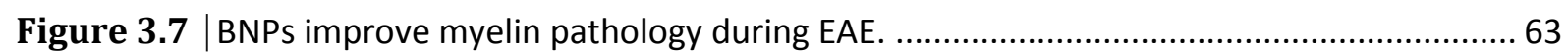

Figure 3.8 | BNPs reduce macrophage infiltration during EAE therapy.......................................... 65

Figure 3.9 | Myeloid cells are essential targets of BNPs in the treatment of EAE. ............................ 67

Figure 3.10 | Combined treatment with BNPs and Dex synergistically improves EAE.....................6 68

Figure 3.11 | Short treatment with BNPs affects glucose metabolism similarly to Dex.................... 70

Figure 3.12 | Short treatment with BNP hardly affects muscle wasting.......................................... 71

Figure 3.13 | Short treatment with BNPs and Dex has a mild effect on gastric emptying ................ 72

Figure 3.14 | Short treatment with BNPs does not affect the osteoporosis marker osteocalcin....... 73

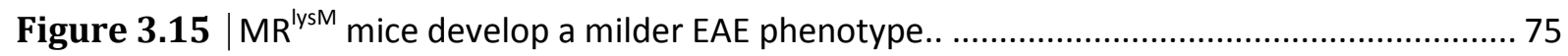

Figure 3.16 | Macrophage infiltration into the spinal cord is reduced in $M R^{l y s M}$ mice during EAE..... 77

Figure 3.17 | Demyelination and axonal damage in $M R^{l y s M}$ mice during EAE are not as severe as in $M R^{f l}$ mice. 78 
Figure 3.18 | The percentage of inflammatory monocytes in blood and spinal cord of $\mathrm{MR}^{\mathrm{lysM}}$ mice is reduced during EAE.

Figure 3.19 | Inflammatory monocytes and neutrophils are unaffected in secondary lymphoid organs of $M R^{l y s M}$ mice during EAE..

Figure 3.20 | MR-deficient BMDMs secrete less M1 cytokines, whereas M2 cytokine release is increased.

Figure 3.21 $\mid \mathrm{MR}^{\mathrm{lysM}} \mathrm{BMDMs}$ stimulate $\mathrm{MOG}_{35--55}$-specific stimulation of $2 \mathrm{D} 2 \mathrm{~T}$ cell proliferation in vitro.

Figure 3.22 $\mid T$ cell priming in $M R^{l y s M}$ mice in the early phases of EAE is unaffected. 86

Figure 3.23 | Secretion of pro-inflammatory cytokines by effector T cells is reduced in the secondary lymphoid organs of $M R^{1 y s M}$ mice before the onset of EAE.

Figure 3.24 | The frequency of Treg cells in peripheral lymphoid organs is increased in $\mathrm{MR}^{\mathrm{lysM}}$ mice..

Figure 3.25 | The frequency of reactive microglia in the spinal cord of $M R^{l y s M}$ mice is reduced. 91 


\section{LIST OF TABLES}

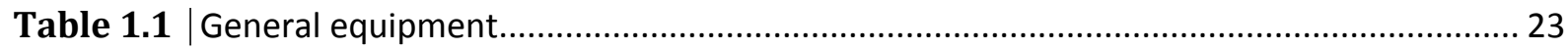

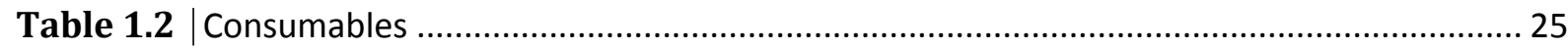

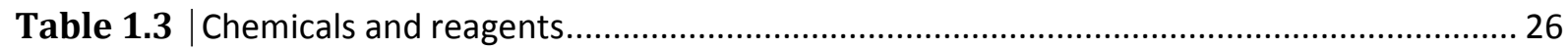

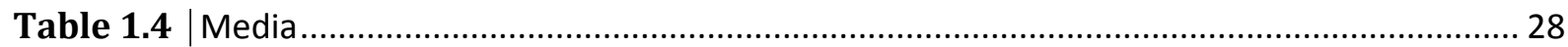

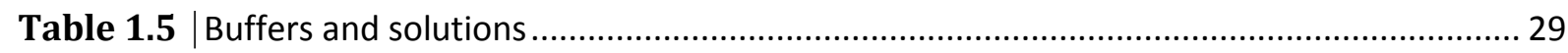

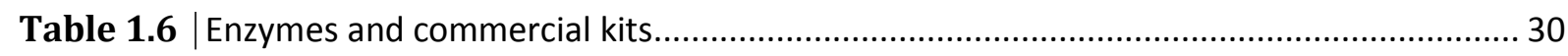

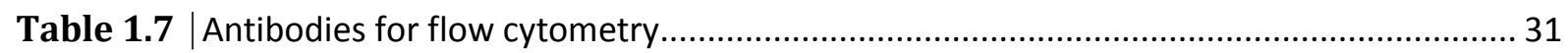

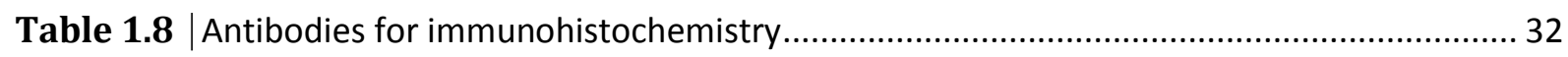

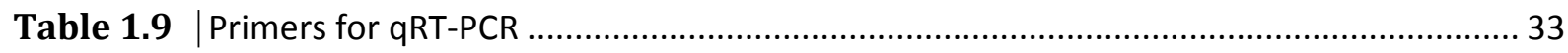

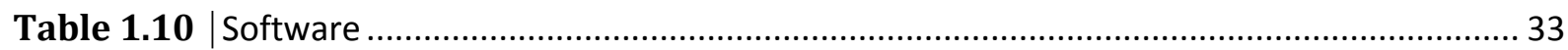

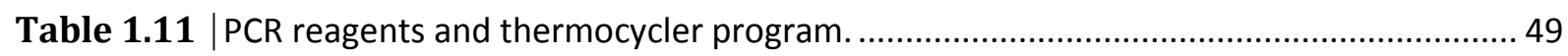

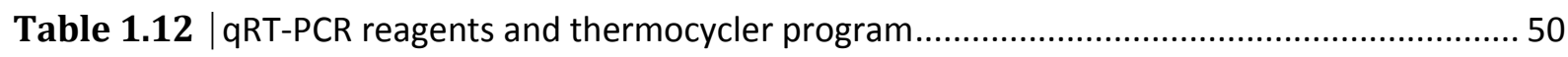




\section{ABBREVIATIONS}

\begin{tabular}{|c|c|}
\hline $11 \beta-H S D \|$ & 11-Beta hydroxisteroid dehydrogenase type II \\
\hline 2-PM & 2-photon microscopy \\
\hline Abs & Antibodies \\
\hline AIA & Adjuvant-induced experimental arthritis \\
\hline AP-1 & Activator protein 1 \\
\hline APC & Antigen presenting cell \\
\hline APC & Allophycocyanine \\
\hline Arg-1 & Arginase 1 \\
\hline BBB & Blood Brain Barrier \\
\hline $\mathrm{Bcl}-2$ & B cell lymphoma 2 \\
\hline BMDM & Bone marrow-derived macrophage \\
\hline BMZ & Betamethasone \\
\hline BNP & Betamethasone nanoparticle \\
\hline CBA & Cytometric bead assay \\
\hline $\mathrm{CCL}$ & CC chemokine ligand \\
\hline$C D$ & Cluster of differentiation \\
\hline CCR & CC chemokine receptor \\
\hline cDNA & Complementary DNA \\
\hline CFA & Complete Freund's adjuvant \\
\hline CFSE & Carboxyfluorescein succinimidyl ester \\
\hline CNS & Central nervous system \\
\hline CSF & Cerebrospinal fluid \\
\hline CXCL & CXC chemokine ligand \\
\hline CXCR & CXC chemokine receptor \\
\hline DC & Dendritic cell \\
\hline $\mathrm{ddH}_{2} \mathrm{O}$ & Double-distilled water \\
\hline Dex & Dexamethasone \\
\hline DMEM & Dulbecco modified Eagle's medium \\
\hline DNA & Deoxyribonucleic acid \\
\hline
\end{tabular}


EAE

ELISA

EMA

ENP

FACS

FDA

FITC

FMN

FoxP3

g

GA

GFP

GC

GILZ

GM-CSF

GR

GRE

h

HRP

ICAM-1

IFNy

Ig

IHC

IL-

iNOS

IOH-NPS

i.p.

i.v.

IVC

IVMP

LCCM

LFB-PAS

LN

LPS

M
Experimental autoimmune encephalomyelitis

Enzyme linked immunoadsorbent assay

European Medicines Agency

Empty phosphate nanoparticles

Fluorescence-activated cell sorting

Food and drug administration

Fluorescein isothiocyanate

Flavin mononucleotide

Forkhead box P3

Gram

Glatiramer acetate

Green fluorescent protein

Glucocorticoid

Glucocorticoid-induced leucine zipper

Granulocyte-macrophage colony stimulating factor

Glucocorticoid receptor

Glucocorticoid responsive element

Hour

Horseradish peroxidase

Intercellular adhesion molecule 1

Interferon gamma

Immunoglobuline

Immunohistochemistry

Interleukine

Inducible nitric oxide synthase

Inorganic-organic hybrid nanoparticles

Intraperitoneal

Intravenously

Individually ventilated cages

Intravenous methylprednisolone

L-929 conditioned medium

Luxol fast-blue periodic acid-Schiff

Lymph node

Lipopolysaccharide

Molar 


\begin{tabular}{|c|c|}
\hline MACS & Magnetic-activated cell sorting \\
\hline MBP & Myelin basic protein \\
\hline MCP-1 & Monocyte chemoattractant protein 1 \\
\hline MFI & Mean fluorescence intensity \\
\hline $\mathrm{MHC}$ & Major histocompatibility complex \\
\hline $\min$ & Minute \\
\hline $\mathrm{ml}$ & Milliliter \\
\hline MM & Meningeal macrophage \\
\hline MMP & Matrix metalloproteinase \\
\hline MR & Mineralocorticoid receptor \\
\hline MRI & Magnetic resonance imaging \\
\hline mRNA & Messenger ribonucleic acid \\
\hline M-CSF & Macrophage colony-stimulating factor \\
\hline MOG & Myelin oligodendrocyte glycoprotein \\
\hline MS & Multiple sclerosis \\
\hline NFKB & Nuclear factor $\mathrm{kB}$ \\
\hline NO & Nitric oxide \\
\hline $\mathrm{O} / \mathrm{N}$ & Overnight \\
\hline PBS & Phosphate buffered saline \\
\hline PCR & Polymerase chain reaction \\
\hline $\mathrm{PE}$ & Phycoerythrin \\
\hline PEG & Polyethylen glycol \\
\hline PFA & Paraformaldehyde acid \\
\hline PL & Liposomal prednisolone \\
\hline PPMS & Primary progressive MS \\
\hline PRMS & Progressive relapsing MS \\
\hline PRR & Pattern-recognition receptor \\
\hline PVM & Perivascular macrophage \\
\hline qRT-PCR & Quantitative real time PCR \\
\hline ReMed & Re-stimulation medium \\
\hline RFP & Red fluorescent protein \\
\hline ROS & Reactive oxygen species \\
\hline RRMS & Relapsing-remitting MS \\
\hline RT & Room Temperature \\
\hline SEM & Standard error of the mean \\
\hline
\end{tabular}


s.c.

SFP

SPMS

Th cell

TLR

TNF $\alpha$

Treg cell

VCAM-1

wt

$\mathrm{ZrO}_{2}$
Subcutaneously

Specific pathogen-free

Secondary progressive MS

T helper cell

Toll-like receptor

Tumor necrosis factor alpha

Regulatory T cell

Vascular adhesion molecule 1

Wild type

Zirconium oxide 


\section{INTRODUCTION}

\subsection{Multiple Sclerosis: the paradigm of CNS autoimmunity}

In the beginning of the 20th century, Paul Ehrlich (awarded in 1908 with the Nobel Prize for his work in immunology) described for the first time the responses of the immune system to its own organism, what he defined as 'horror autotoxicus'. His observations set the basis of the concept of autoimmunity (Ehrlich P. and Morgenroth J. 1957).

Autoimmune responses arise as a consequence of the breakdown of self-tolerance, a process tightly regulated in the lymphoid organs. Once self-reactive lymphocytes escape central tolerance in the thymus or bone marrow, the mechanisms of peripheral tolerance, like regulatory immune cells, become crucial to prevent exacerbated immune responses against self antigens. Failure of these regulatory mechanisms due to genetic factors or external triggers alters innate immunity and results in the development of autoimmune diseases.

Certain organs, defined as immune privileged, lack conventional lymphatic vasculature and are delimited by functional and anatomical barriers that restrict the access of immune cells. One example of immune privileged organ is the central nervous system (CNS), which is surrounded by the blood-brain barrier (BBB). Although nowadays the view of the CNS as immune privileged is in question (Carson et al. 2006; lliff et al. 2015), for many years this organ was believed to be exempt from immune responses (Medawar 1948; Barker and Billingham 1972). However, in some cases the brain also becomes target of auto-reactive immune cells. CNS antigens are constantly released to induce peripheral tolerance (Harris et al. 2014), but if antigen-specific lymphocytes recognize them as non-self and get activated, these antigens are able to induce changes in the BBB permeability to enter the CNS, thereby starting an inflammatory process that may result in autoimmunity. This situation is clearly reflected by the autoimmune pathology in multiple sclerosis (MS). 


\subsubsection{Multiple Sclerosis}

MS is the most common inflammatory disease of the CNS and is characterized by focal myelin loss and axonal damage within the white matter. This neurodegenerative process leads to severe motor, sensory and cognitive deficits. The disorder affects around 2.5 million people worldwide (Compston and Coles 2002), with a particularly increasing prevalence in North America and Europe, where $>100 / 100.000$ inhabitants suffer from it (Leray et al. 2016). The first symptoms, which include blurred vision and muscle weakness, normally appear between 20 and 40 years of age, and the disease is two times more prevalent in females than in male individuals.

It was Jean Martin Charcot who in 1868 first described the symptomatology associated with sclerotic plaques in patients with neurologic dysfunction, although those neuroanatomic observations had already been depicted years before by Robert Carswell (1838) and Jean Cruveilhier (1842). MS lesions are defined by four histopathological features: inflammation, astrogliosis, demyelination and axonal loss, the latter being the direct cause of the neurological disabilities in MS patients. Nevertheless, depending on the patient and the disease stage the lesions can present with different demyelination patterns, and the contribution of distinct immune infiltrates may vary (Lucchinetti et al. 2000; Lassmann et al. 2007). This complexity is also reflected by the high inter-individual variability of symptoms and disease progression. Based on that, patients can be grouped in four different modalities of MS (Hauser and Goodwin 2008):

- Relapsing-remitting MS (RRMS): this is the most prevalent form of MS (85\% of the cases), in which patients suffer from sporadic episodes of neurological impairment with full or partial recovery. In this phase of the disease, inflammatory infiltrates are the major component of the lesion.

- Secondary progressive MS (SPMS): also characterized by a relapsing fashion but with persistence of disability after each relapse with periods of no remission. It often develops in patients with a previous history of RRMS, and at this stage inflammation gives its leading role up to a more prominent demyelinating pathology and the subsequent axonal damage. 
- Primary progressive MS (PPMS): affects $10 \%$ of MS patients and is the most severe variant due to its unresponsiveness to conventional therapies. These patients accumulate deficits progressively from the beginning of the disease, and no remission phases occur.

- Progressive relapsing MS (PRMS): this rare form of MS shares the progressive course of PPMS with flare-ups that lead to acute worsening of the symptoms.

Despite the efforts made to determine the causes of MS, little is known about the etiology of the disease. As other autoimmune disorders, MS is believed to arise from a combination of genetic and environmental factors. Among all possible environmental triggers, epidemiological studies show that high-salt diet, Vitamin D deficiency and smoking are most relevant (Kleinewietfeld et al. 2013; Hedström et al. 2015; Hucke et al. 2016; Mimura et al. 2016). Some bacterial and viral infections have also been linked to MS, particularly infection with Epstein-Barr virus (Fernández-Menéndez et al. 2016). Apart from the external factors, the higher incidence of MS among relatives suggests a genetic component of the disease (Sadovnick 1993). Different polymorphisms of genes involved in immune responses have been identified as risk factors in genome-wide association studies. The strongest association with a higher MS susceptibility was found in the DRB1*15:01 allele of the human leukocyte antigen (HLA) complex (Sawcer et al. 2011), but also specific allelic variants of genes coding for cytokines receptors (IL2RA and IL7R) or co-stimulatory molecules (CD58, CD80 and CD86) have been reported to increase the predisposition to MS (Hafler et al. 2007; Sawcer et al. 2011; Traboulsee et al. 2014).

\subsubsection{Animal models of MS - Experimental autoimmune encephalomyelitis}

The complexity and the wide heterogeneity of MS make it a difficult matter of study. Nevertheless, throughout the years several rodent and non-human primate models resembling different aspects of MS pathology have provided useful information for the understanding of the disease, and have been essential tools for the development of the current MS therapies. 
Damage of the myelin sheaths of the nerves followed by only partial re-myelination is a hallmark of MS. To study the mechanisms involved in myelin loss and regeneration, there are several animal models available based on toxic or viral agents. Toxic-induced demyelination is achieved with lysolecithin or cuprizone, a copper chelating compound that induces oligodendroglial cell death, affecting almost exclusively this cell type (Matsushima and Morell 2001). As an alternative, neurotropic viral infection with Theiler's murine encephalitis virus (TMEV) has the advantage of including the inflammatory component typical from MS, as CNS infiltrates are found in the TMEV-infected mice (Tsunoda et al. 2006).

Despite not being the model of choice for demyelination studies, experimental autoimmune encephalomyelitis (EAE) is most extensively employed and mimics more aspects of the autoimmune pathology from MS patients. This model was developed by Rivers and colleagues using monkeys that were immunized with rabbit brain extracts, which resulted in paralysis associated with perivascular infiltrates and demyelination (Rivers et al. 1933). Nowadays, the immunogens used for EAE induction in rodents and non-human primates are components of the myelin sheaths, mostly myelin basic protein (MBP), proteolipid protein (PLP), myeloid oligodendrocyte glycoprotein (MOG), or peptides derived from the latter one. The disease course is characterized by progressive paralysis from the tail up to the forelimbs (Berard et al. 2010), and can be induced in different mouse and rat strains either via active immunization or adoptive transfer of encephalitogenic $T$ cells. Similarly to the human disease, the progression pattern is highly variable: depending on the genetic background and the immunizing agent, EAE can follow a relapsing-remitting course (Lublin et al. 1981) or develop in a chronic-progressive way (Tuohy et al. 1989).

Noteworthy, EAE cannot be properly induced without the help of adjuvants. Complete Freund's Adjuvant (CFA), an oily solution containing heat-inactivated M. tuberculosis (Freund et al. 1947), induces Th1 responses thereby providing an immunological environment that favors the initiation of the disease (Smith et al. 2011). Additionally, Pertussis toxin facilitates the permeabilization of the $B B B$, and has other boosting effects like increasing adhesion molecules in lymphocytes, inducing maturation of dendritic cells (DCs) and suppressing regulatory T cells (Treg cells) (Hou et al. 2003; Kerfoot et al. 2004; Chen et al. 2006). 
The EAE model has been a valuable tool to study MS pathomechanisms, nevertheless EAE is an artificially generated entity that differs in many aspects from human MS (Procaccini et al. 2015). First of all, the use of inbred strains does not reflect the complex human heterogeneity. Furthermore, disease initiation is controlled and induced under the effect of strong adjuvants, thus we cannot obtain information about the natural causes of MS. Another limiting factor is that inflammation and demyelination in EAE are restricted to the spinal cord white matter, while MS lesions are mostly found in the brain and cerebellar cortex. And moreover, EAE is mainly driven by $\mathrm{CD}^{+} \mathrm{T}$ cells, and little information about the role of $\mathrm{CD}^{+} \mathrm{T}$ cells or $\mathrm{B}$ cells can be obtained. Some of these issues were addressed in the recent years thanks to alternative EAE models with transgenic animals and different induction protocols (Litzenburger et al. 1998; Ford and Evavold 2005; Bettelli 2007); nevertheless, new approaches are still needed to answer these questions.

\subsection{The cellular players of CNS autoimmune inflammation}

Although the mechanisms that initiate MS in humans remain elusive, many years of study on EAE models have provided a quite accurate picture of how the disease starts in rodents. In rough outlines, myelin peptides derived from the immunizing agents are presented by DCs in the lymph nodes and stimulate auto-reactive T cells that have escaped negative selection in the thymus. Afterwards, the autoantigen-specific activated T cells proliferate quickly and circulate until they encounter their cognate antigen in the CNS. Once there, they promote the open up of the BBB and secrete cytokines and chemokines that attract subsequent waves of immune cells, including more T cells, B cells and myeloid cells. The chronic inflammatory response established in the spinal cord damages the myelin cover of the nerves and, in some EAE models, eventually disrupts axonal structures. All this leads to the described symptomatology in the animals.

In summary, MS and EAE are complex entities where the interplay of different immune cells, belonging to both the innate and the adaptive immune system, determines their progression and final outcome (Figure 1.1). The specific roles of the key cell mediators of these CNS autoimmune reactions will be described below in more detail. 


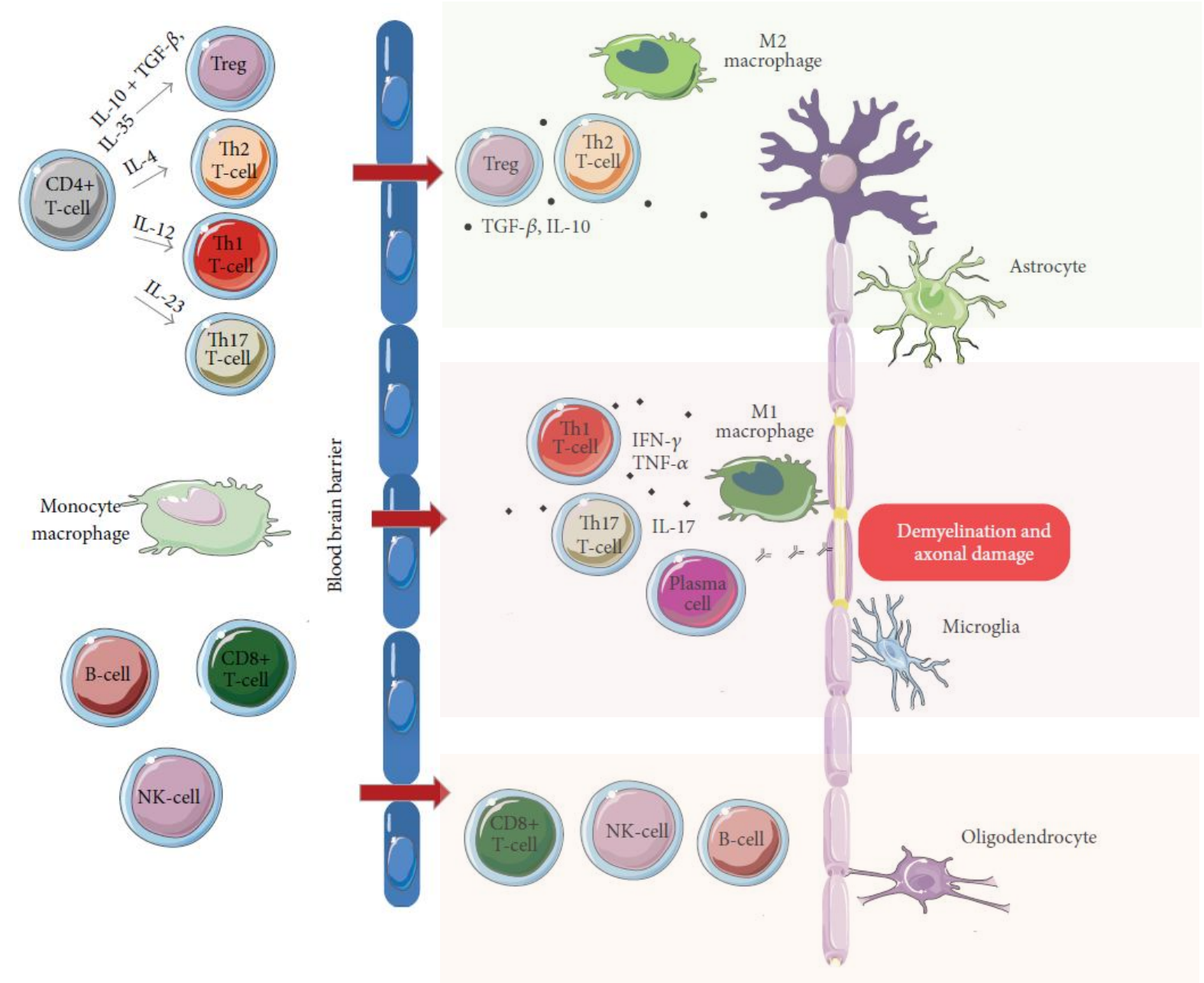

Figure 1.1 | Immune cells participating in MS and EAE pathology. In the initial stages of EAE, soluble mediators secreted by auto-reactive T cells facilitate the opening of breaches in the BBB, allowing the access of different immune cell types to the CNS. These cells can be subdivided into three groups according to their contribution to the disease. The first group consists of effector cells, with the mission of promoting inflammation, and includes Th1, Th17, antibody-secreting plasma cells and M1 macrophages. Another group of cells fulfill regulatory functions, important to control the exacerbation of the immune response; to this section belong Th2 cells, Treg cells, and M2 macrophages. Finally, the third part of this classification refers to cells with both inflammatory and regulatory activities, the roles of which in the context of EAE have not been fully elucidated yet: these are $\mathrm{CD} 8^{+}$cells, NK cells and B cells. Beyond infiltrating leukocytes, also cells residing in the CNS, like microglia, astrocytes and oligodendrocytes, contribute to MS and EAE progression. Adapted from Duffy et al. 2014. 


\subsubsection{T cells functions in MS and EAE}

MS and EAE have always been described as T cell-driven diseases. Evidence for this is that adoptive transfer of encephalitogenic T cells isolated from immunized mice is capable of inducing a strong disease course in healthy animals (Ben-Nun et al. 1981; Zamvil et al. 1985). T cells are, together with B cells, the major effectors of the adaptive immune response. Depending on the class of the MHC molecules encountered during thymic selection, naïve T cells can adopt two major phenotypes. On the one hand, T cells expressing the CD4 coreceptor, denominated T helper (Th) cells, are in charge of modulating immune responses via expression of membrane-bound molecules and secretion of cytokines and chemokines. Upon encounter with their cognate antigen (phenomenon defined as priming), signals provided by the antigen-presenting cell (APC) will determine their further differentiation into the Th1, Th2 or Th17 subsets, each one with specific effector functions (Mosmann et al. 1986; Langrish et al. 2005). On the other hand, $\mathrm{CD}^{+} \mathrm{T}$ cells are specialized in eliminating harmful target cells, e.g. virally-infected cells, via direct cytotoxicity. Although both T cell types can be found in the CNS of animals with EAE, apparently $C D 4^{+} \mathrm{T}$ cells are the main mediators of the disease. In contrast, in human $\mathrm{MS} \mathrm{CD} 8^{+} \mathrm{T}$ cells are generally more abundant in the CSF of the patients (Koh et al. 1992; Babbe et al. 2000).

Since interferon gamma (IFNY)-secreting cells were present in the CNS of mice after immunization with MBP (Ando et al. 1989; Renno et al. 1994), EAE was initially considered a Th1-mediated pathology. In agreement with the Th1/Th2 dichotomy, Th2 cells (characterized by the production of IL-4, IL-5 and IL-13) were then proposed to protect from EAE (Adorini et al. 1996). This notion was confronted by other publications showing that TCR-engineered Th2 cells were also capable of inducing autoimmune CNS responses (Lafaille et al. 1997). Th1 cells secrete a cytokine profile that includes tumor necrosis factor alpha (TNF $\alpha$ ) and IFNy. Despite the active participation of these cytokines during neuroinflammation, their contribution to $E A E$ and $M S$ is under debate. Certain experiments performed with TNF $\alpha$-deficient mice showed a delayed EAE onset and reduced infiltration into the CNS parenchyma (Körner et al. 1997), whereas other investigations pointed to a dispensable role of TNFa for EAE initiation and severity (Kassiotis et al. 1999; Batoulis et al. 2011). The contribution of IFNy to EAE and MS is equally controversial. Blockade of IFNy, but 
not TNF $\alpha$, reduced the number of active lesions in patients with SPMS (Skurkovich et al. 2001). Additionally, MS treatment with IFNy resulted in disease exacerbation (Panitch et al. 1987). However, in the late 90s several studies showed that IFNy deficiency aggravated the course EAE, suggesting a protective effect of the molecule (Ferber et al. 1996; Krakowski and Owens 1996; Chu et al. 2000). This hypothesis was further investigated with similar outcome in mice devoid of IL-12, the cytokine responsible of Th1 differentiation, or its different subunits (Becher et al. 2002; Gran et al. 2002; Zhang et al. 2003). But surprisingly, the deletion of the IL-12p40 subunit rendered the mice resistant to EAE, revealing the essential role of a new cytokine: IL-23 (Oppmann et al. 2000; Becher et al. 2003; Cua et al. 2003). IL-23 shares with IL-12 the p40 subunit and is responsible for the stability of a different Th phenotype, the Th17 subset (Langrish et al. 2005). These findings drastically changed the view of EAE as a Th1-driven disease and established the new lines of EAE research.

Th17 cells are now believed to be the initiators of EAE (Korn et al. 2007a). These cells are defined by the production of cytokines from the IL-17 family, especially IL-17A and IL-17F, although they secrete IL-6, IL-22, TNF $\alpha$ and GM-CSF as well. IL-17 exerts different activities during the early phases of EAE. First, it acts locally on the near tissues inducing the expression of cytokines and chemokines that are important for the mobilization and migration of myeloid cells to the inflamed CNS (e.g. CCL-7 and CCL-2). Moreover, IL-17 has a direct impact on the integrity of the BBB by disorganizing tight-junctions, inducing reactive oxygen species (ROS) and increasing the expression of adhesion molecules, such as ICAM-1, by endothelial cells (Huppert et al. 2010). Deletion of IL-17 or its receptor has been reported to significantly improve disease progression (Komiyama et al. 2006), however, it does not result in resistance to EAE (Haak et al. 2009; Kroenke and Segal 2011).

Noteworthy, Th17 cells have the ability to switch their phenotype to the Th1 subtype once they reach the CNS, and this plasticity appears to be controlled by IL-23 (Kurschus et al. 2010; Hirota et al. 2011). Hence, Th1 and Th17 responses complement each other rather than competing during EAE (Stromnes et al. 2008), and therefore the simultaneous secretion of IFNy and IL-17 can be found in the CNS of animals with EAE (Abromson-Leeman et al. 2009). The common denominator between these two Thelper subsets is GM-CSF, a cytokine produced downstream to IL-23 that has been identified as the only molecule produced by T 
cells strictly necessary for the development of EAE (Codarri et al. 2011; El-Behi et al. 2011). Probably the importance of this cytokine resides in its function as link between $T$ cells and myeloid cells, since it promotes the release of myeloid progenitors from the bone marrow and influences the differentiation and polarization of cells from both immune cell types.

But not all T cell activities are detrimental for the CNS. The balance between effector and regulatory functions is essential to prevent autoimmune disorders, and to maintain this balance the adaptive immune system relies on Treg cells. This additional $\mathrm{T}$ cell subpopulation is originated either by thymic selection (naturally occurring Treg cells, nTreg cells) or in peripheral lymphoid organs during priming of naïve T cells (inducible Treg cells, iTreg cells). Induction of iTreg cells is strongly influenced by the cytokine milieu, and TGF $\beta$ is necessary for this differentiation. Importantly, TGF $\beta$ in combination with IL-6 also participates in the induction of Th17 cells, meaning that presumably the presence or absence of IL- 6 determines the differentiation of naive $\mathrm{CD}^{+}{ }^{+} \mathrm{T}$ cells into either the Th17 or the Treg cell compartment (Xu et al. 2007; Korn et al. 2009). Moreover, the local environment may direct the conversion between both cell types (Xu et al. 2007; Koenen et al. 2008) and, in fact, some publications claimed that part of the Treg cells found in the CNS of mice with EAE originated from encephalitogenic T cells (Liu et al. 2006).

Treg cells are generally characterized by the expression of the phenotypic markers CD4, IL-2 receptor $\alpha$-chain $(C D 25)$ and the transcription factor FoxP3. Their potent suppression of immune effector functions is achieved via different mechanisms: first, indirect suppression via secretion of regulatory cytokines, such as IL-10, IL-35 and TGF $\beta$, and second, cell-to-cell contact with effector T cells resulting in disrupted TCR-induced proliferation and reduced IL2 secretion (Groux et al. 1997; Thornton and Shevach 2000; Sakaguchi et al. 2008).

Given the importance of Treg cells for the maintenance of tolerance, it was not surprising to find dysfunctional Treg cells in MS patients and mice with EAE (Viglietta et al. 2004; Haas et al. 2005). Treg cells have been shown to prevent the development of CNS inflammation and ameliorate EAE disease course (Kohm et al. 2003). Additionally, it has been described that during the remission phase of MS and EAE the number of Treg cells increases (Korn et al. 2007a; O'Connor et al. 2007), which highlights their participation in disease resolution. 


\subsubsection{The role of myeloid cells in CNS autoimmunity}

The inflammatory activities of T cells would not be possible without the help of myeloid cells. Myeloid cells constitute the first line of defense against infections and moreover, establish the connection between innate and adaptive immune responses. These cells are especially relevant in $E A E$, since they recognize components of the adjuvants employed for immunization via pattern recognition receptors (PRRs), e.g. Toll-like receptors (TLRs) and provide a pre-activated cytokine milieu that is necessary for disease induction (Imrich and Harzer 2001).

All myeloid cells derive from a pluripotent common myeloid progenitor in the bone marrow, and differentiate into the different lineages (DCs, monocytes/macrophages and granulocytes) in response to signals from peripheral tissues. In the context of MS and EAE the three subsets of myeloid cells fulfill essential functions in the sequence of events leading to CNS inflammation. DCs, defined by the expression of CD11c and considered professional APCs, are the first ones to present myelin antigens to naïve T cells in the lymph nodes. Thus, fully functional DCs are strictly required for EAE initiation (Greter et al. 2005; Hertzenberg et al. 2013). After activation and migration of Th17 cells, neutrophils (a granulocyte subtype characterized by the surface expression of Ly6G) are the next line of immune mediators. In SJL and Balb/C mice, impaired neutrophil migration to the CNS or treatment with neutrophildepleting antibodies (Abs) resulted in delayed EAE onset and attenuated disease severity

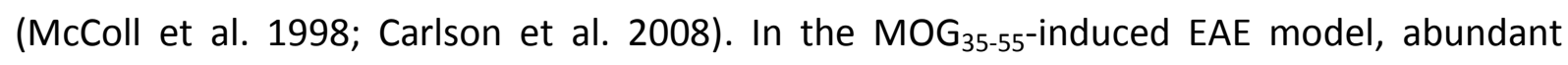
$\mathrm{Ly}_{6 \mathrm{G}}{ }^{+}$infiltrates were found in the perivascular space during the asymptomatic phase of the disease (Soulika et al. 2009). It is known that just before the onset of clinical symptoms, neutrophils are able to permeabilize the BBB via secretion of matrix metalloproteinase (MMPs) (Folgueras et al. 2008; Soulika et al. 2009) and are neurotoxic in a contactdependent and paracrine manner (Dinkel et al. 2004). In addition, because of their production of pro-inflammatory cytokines, neutrophils promote DC and monocyte maturation within the CNS, thereby supporting APC functions (Steinbach et al. 2013).

From all myeloid cells, the monocyte/macrophage compartment (defined by the expression of the phenotypic marker $(D 11 b)$ is particularly interesting for MS and EAE, since it participates in all stages of disease progression either in an inflammatory or regulatory way. 
Furthermore, they are the only immune cells with permanent representation within the CNS, where perivascular macrophages (PVMs), meningeal macrophages (MMs) and microglia are located.

Produced under the effect of GM-CSF in the bone marrow, monocytes circulate in blood and secondary lymphoid organs, and express chemokine receptors and adhesion molecules that mediate their infiltration into the inflamed tissue. There, they can differentiate into macrophages or DCs depending on the cytokine environment and the activation of PRRs.

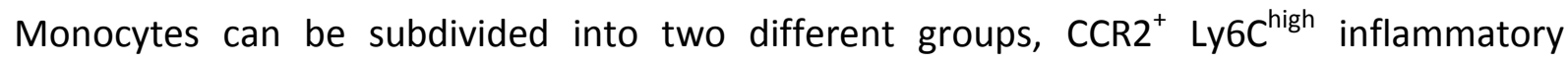
monocytes, and CX3CR1 ${ }^{+}$Ly6C ${ }^{\text {low }}$ resting ones (Geissmann et al. 2003). It has been shown that deletion of CCR2 from these myeloid cells strongly ameliorates EAE clinical symptoms, which reveals a crucial role of inflammatory monocytes in CNS autoimmunity (Mildner et al. 2009).

Macrophages, due to their prominent phagocytic capacity, are essential elements of the host defense against pathogens, but also fulfill important homeostatic functions by clearing cellular debris allowing tissue remodeling (Mosser and Edwards 2008). Hence they function in a dual manner in the context of MS as well, contributing to both tissue injury and repair. Macrophages derive from blood monocytes (van Furth et al. 1972) that migrate to inflammatory sites following chemokine-traced paths. Some monocytes also replenish tissue-resident macrophages, populations that function as immune sentinels in specific niches. The main feature of macrophages is their remarkable plasticity. They have the appropriate receptor machinery to sense a wide diversity of signals and dynamically change their phenotype accordingly. Mirroring the Th1/Th2 classification, initially these phenotypes were divided into classically activated macrophages (M1) and alternatively activated ones (M2). This terminology, however, didn't reflect the real heterogeneity of macrophage activation. Nowadays, a full spectrum of polarization states ranging from $M 1$ to $M 2$ has been proposed, and every intermediate state should be independently defined depending on the specific activating stimuli (Mosser and Edwards 2008; Martinez and Gordon 2014; Hume 2015). In a simplified way (Figure 1.2), M1 polarization is primarily induced by INFY, TNFa and GM-CSF coming from Th1 cells, NK cells or macrophages themselves. Recognition of bacterial elements by PRRs, e.g. LPS through TLR4, can also trigger macrophage classical 
activation. These stimuli promote elimination of pathogens by increasing phagocytosis and antigen presentation, a process that involves up-regulation of $\mathrm{MHC}$ class II and the costimulatory molecules CD80 and CD86. M1 macrophages also enhance their expression of the inducible nitric oxide synthase (iNOS, NOS2) and secrete pro-inflammatory cytokines such as IL-1 $\beta$, IL-6, IL-12, IL-23 and TNF $\alpha$. Conversely, alternative macrophage activation by IL-4 and IL-13 supports clearance of parasites via expression of the mannose receptor (CD206), an important mediator of endocytosis, and secretion of polyamines. Additional markers of this macrophage phenotype are Arginase-1 (Arg-1), Fizz1 and Ym1 (Rőszer 2015). In parallel, IL-10 and glucocorticoids (GCS) have the ability to induce a different alternative phenotype involved in immune regulation and tissue remodeling, defined by Mantovani as M2c or deactivated state (Mantovani et al. 2004; Kleiman et al. 2012; Rőszer 2015). This phenotype is characterized by the expression of IL-10, TGF $\beta$, CD206 and CD163.

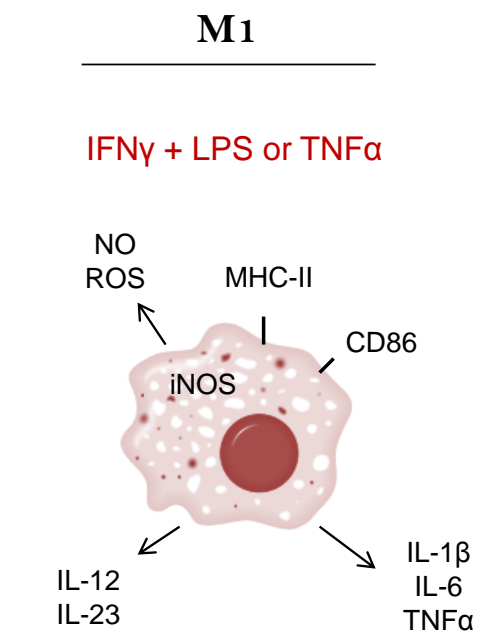

Classical (M1)

Type I Inflammation

Killing of intracellular pathogens

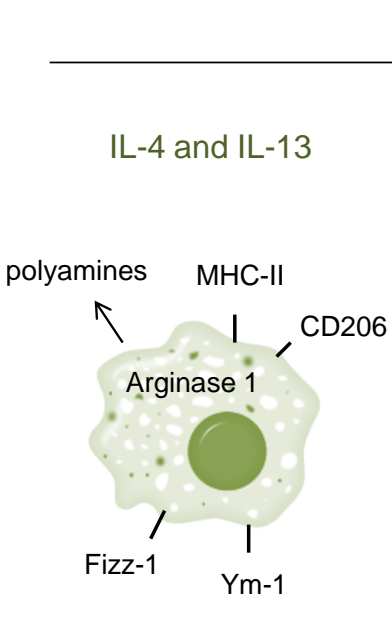

Alternative (M2a)

Type II inflammation

Killing of parasites

M2

IL-10

Glucocorticoids

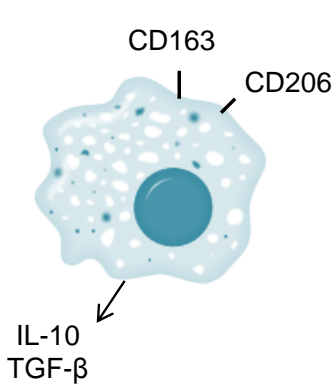

Deactivated (M2c)

Immunoregulation

Tissue remodelling

Figure 1.2 | Macrophage polarization induced by different stimuli. Classification of macrophage activation states according to Mantovani et al. The figure depicts the specific inducing stimuli, typical markers and cytokines, as well as immune functions of each macrophage phenotype. Adapted from Mantovani et al. 2004. 
The participation of different subsets of macrophages during MS and EAE has been extensively reported. It is known that the number of monocyte-derived pro-inflammatory macrophages increases progressively in the CNS until the peak of the disease (Mikita et al. 2011). In this stage of CNS inflammation, however, macrophages are difficult to be distinguished from microglia, their counterparts in the CNS parenchyma. Microglia, in contrast to infiltrating monocyte-derived macrophages, derive from myeloid precursors originating in the yolk sac that populate the CNS during embryonic development (Kierdorf and Prinz 2013). After birth, these populations are self-sustained by local progenitor cells (Ajami et al. 2011). All in all, activated macrophages/microglia play an important detrimental role at disease onset and are a prominent component of active demyelinating MS lesions (Barnett and Prineas 2004). In the first place, microglia are the major source of chemokines for $\mathrm{T}$ cell recruitment. Peripheral macrophages do not seem to be involved in this $\mathrm{T}$ cell chemoattraction, since their depletion with clodronate liposomes does not impair $\mathrm{T}$ cell migration (Tran et al. 1998). However, in mice where macrophages were depleted, T cells could not access the CNS parenchyma and EAE induction was abrogated (Huitinga et al. 1990; Tran et al. 1998), probably due to the lack of MMPs (Toft-Hansen et al. 2004). Additionally, macrophages/microglia up-regulate MHC II molecules and secrete proinflammatory cytokines during neuroinflammation, thereby collaborating in the reactivation of T cells (Murphy et al. 2010). These cytokines, in combination with ROS, NO and glutamate are also neurotoxic, leading to axonal damage and oligodendroglial death (Shijie et al. 2009).

Nevertheless, the alternatively-activated variants of these myeloid cells have a regulatory function during $\mathrm{MS}$ and EAE. The presence of $\mathrm{M} 2$ macrophages/microglia has been associated with disease resolution (Mikita et al. 2011), and these cells have been shown to contribute to neuronal regeneration via secretion of neurotrophic factors (Elkabes et al. 1996; Batchelor et al. 1999). These cells also participate in the removal of myelin debris, which are known to be neurotoxic and to inhibit re-myelination (Kotter 2006). Moreover, several publications correlate the benefits of M2 polarized macrophages with increased Treg cell-mediated immunosuppression (Weber et al. 2007; Keating et al. 2009) 


\subsection{Immunomodulatory therapies for MS: glucocorticoids}

Up to now there is no cure for MS. Nevertheless, in the past 20 years the better understanding of the disease pathomechanisms provided by animal models has led to the development of different therapies approved by the Food and Drug Administration (FDA) and the European Medicines Agency (EMA). Most of these drugs, so called diseasemodifying therapies (DMTs), are immunomodulatory compounds that have proven to successfully reduce MS relapse rate and slow down the development of neuronal damage associated with disease progression, especially in the early phases of RRMS. Classical first line therapies for RRMS patients are IFN $\beta$ (Paty and Li 1993) and glatiramer acetate (GA) (Johnson et al. 1995); in the case of patients not responding to these drugs, the use of the monoclonal antibodies Natalizumab (Polman et al. 2006) and Alemtuzumab (Brown and Coles 2013) is recommended. Despite the efficacy of IFN $\beta$ and GA, their application via subcutaneous (s.c.) or intramuscular (IM) injection often affects patient compliance; to solve this problem, in recent years new oral drugs like dimethyl fumarate (Gold et al. 2012), teriflunomid (Papadopoulou et al. 2012) and fingolimod (Kappos et al. 2010) have been also approved by the FDA and are currently in use, although the latter just as second line treatment. Unfortunately, finding the appropriate therapy for the progressive forms of the disease is still a challenge for the clinicians. In these cases, the immunosuppressive drug Mitoxantrone is often the treatment of choice in spite of its associated side effects (Boster et al. 2008).

\subsubsection{Glucocorticoid treatment in MS patients}

Complementary to the approved DMTs, high-dose GCs are the first line therapy to treat acute exacerbations of MS. Cortisol, the natural GC in humans, is a steroid hormone produced by the adrenal glands that participates in many different physiological processes, such as embryonic development, response to stress or glucose metabolism. It also has effects on the immune system, e.g. controlling the differentiation and survival of leukocytes. Due to their potent immunosuppressive properties, natural and synthetic GCs have been extensively used during decades for the treatment of numerous inflammatory and 
autoimmune disorders (Hench et al. 1950), like rheumatoid arthritis, allergic asthma, inflammatory bowel disease, MS and psoriasis. Still nowadays, GCs are the most commonly prescribed anti-inflammatory drugs.

Administration of $1 \mathrm{~g}$ /day intravenous (i.v.) methylprednisolone (IVMP) for 3-5 days is the standard treatment for acute relapses of RRMS (Goodin 2014). Acute relapses are defined as episodes longer than $24 \mathrm{~h}$ of neurological impairment caused by active inflammatory demyelinating lesions (McDonald et al. 2001). These episodes are mostly followed by resolution of the symptoms, but unfortunately this is not always the case, and an incomplete recovery is often associated with a worse prognosis (Renoux 2011). Hence, on time and efficient management of the relapses is critical for the patients. Different clinical trials have confirmed the benefits of GCs in terms of relapse recovery (Milligan et al. 1987; Filippini et al. 2000; Miller et al. 2000). Moreover, the results of the "Optic Neuritis Treatment Trial" (Beck et al. 1993) showed that optic neuritis patients receiving high-dose IVMP appeared to have a lower risk of developing MS than the placebo controls, suggesting that GCs might have long-lasting immunosuppressive effects. To shed light on this question some studies tested the administration of GCs over longer time periods. In one of these studies, prolonged administration of low-dose oral GCs did not appear to influence relapse rate or progression of disability (Miller et al. 1961). However, a second trial using similar doses indicated improved neuronal deterioration after 18 months daily treatment (Tourtellotte and Haerer 1965). Using an alternative approach, pulsed high-dose IVMP treatment in patients with RRMS significantly prevented the accumulation of disability (Zivadinov et al. 2001). In accordance with these results, another clinical trial showed that a single monthly dose of 500mg IVMP reduced the number of lesions for 12 months (Then Bergh et al. 2006), and also studies on SPMS patients have reported an improvement in the disease progression with pulsed GC therapy (Goodkin et al. 1998). None of these treatments seemed to influence the frequency of relapse, but in another trial in 2009 this positive effect was indeed observed when pulsed IVMP was applied as an add-on to IFN $\beta$ therapy (Sorensen et al. 2009). Collectively, these studies indicate that pulsed GC therapy might also offer an alternative to interfere with the natural history of the disease, but further research is required to confirm this hypothesis. 


\subsubsection{Molecular and cellular basis of GCs immunosuppression}

The immunosuppressive properties of GCs rely on a complex molecular mechanism. GCs diffuse through the plasma membrane and act predominantly by binding to the GR, which is located in the cytosol in association with chaperones and immunophillins that keep it in an inactive state (Hutchison et al. 1996). After GC binding, the GR is released from these proteins and translocates into the nucleus, where it can act both as a monomer or a dimer. GR homodimers recognize DNA palindromic sequences in promotor or enhancer regions called GC-responsive elements (GREs) (Chandler et al. 1983). GC-activation of these GREs modulate the transcription of genes either positively, e.g. IL-10, GILZ and Bcl-X (D'Adamio et al. 1997; Hodge et al. 1999; Gascoyne et al. 2003), or negatively, e.g. IL-1 $\beta$, osteocalcin and prolactin (Sakai et al. 1988; Strömstedt et al. 1991; Zhang et al. 1997). In contrast, in its monomeric form the GR binds to transcription factors, such as AP-1 or NFKB, that participate in inflammatory signaling cascades, resulting in the trans-repression of pro-inflammatory genes via tethering interactions. Studies with $G R^{\text {dim }}$ mice, in which dimerization of the receptor is impaired (Reichardt et al. 1998), revealed that both mechanisms act separately and fulfill different functions depending on the cell type and the physiological context (Kleiman et al. 2012; Reichardt et al. 2012). In addition, GCs have also rapid non-genomic effects mediated by non-specific interactions with cellular membranes, or with membranebound GRs (Bartholome et al. 2004). However, the consequences of these interactions are still not well understood (Strehl et al. 2011).

Regarding the activities of $\mathrm{GCS}$ in the context of $M S$ and $E A E$, five different immunosuppresive mechanisms have been proposed:

1) GCs have a pro-apoptotic effect on immune cells, especially T cells, involving members of the Bcl-2 family and caspases (Leussink et al. 2001; Tuckermann et al. 2005).

2) Furthermore, they alter the overall inflammatory cytokine milieu. GCs are known to reduce the expression of several pro-inflammatory cytokines, such as TNF $\alpha$, IFNy and IL-2 (Almawi et al. 1996). Apart from direct genomic effects, this inhibition can be partially explained by the simultaneous down-regulation of IL-12 and IL- 6 in myeloid cells, hindering Th1 and Th17 differentiation (Blotta et al. 1997). In contrast, higher levels of IL-10, an 
important cytokine for the negative control of autoimmune responses, were found in MS patients after MP treatment (Gayo et al. 1998).

3) In EAE models, GCs have also been reported to influence T cell chemotaxis via modulation of chemokines like CXCL12 and its receptor CXCR4 (Schweingruber et al. 2014).

4) GCs have the ability to partially restore the BBB, preventing the extravasation of leukocytes into the CNS. These benefits derive from the up-regulation of molecules such as occludine and claudine (Kashiwamura et al. 2011), and down-regulation of adhesion molecules both in leukocytes (LFA-1) and endothelial cells (ICAM-1, VCAM-1) (Pitzalis et al. 2002). The reduced expression of MMPs also contributes to the maintenance of the BBB stability (Rosenberg et al. 1996).

5) Finally, GCs diminish the release of NO and ROS by macrophages and microglia (Lim et al. 2007), directly preventing neurotoxicity. Furthermore, additional positive effects resulting from M2 polarization of these cells have been described (Kiefer and Kreutzberg 1991; Varga et al. 2008).

\subsubsection{Mineralocorticoid receptor-mediated activities of GCs}

It is known that, in certain situations, GCs also activate the mineralocorticoid receptor (MR) via similar molecular mechanisms as the GR. The MR and the GR share 57\% homology in their ligand-binding domain, 94\% in the DNA-binding domain (Funder 1997), and have similar affinities for cortisol (Funder 1993). But in contrast to the ubiquitously present GR, MR expression is confined to certain epithelial tissues such as kidney and colon, some nonepithelial tissues like hippocampus and heart, and specific immune cell subsets, mostly myeloid cells. Furthermore, some of these tissues co-express the $11 \beta$-hydroxysteroid dehydrogenase type II (11ß-HSD II), an enzyme that locally inactivates GCs, making the MR mostly sensitive to aldosterone instead of cortisol and costicosterone. Aldosteronemediated effects of the MR are, for instance, the regulation of electrolyte homeostasis in the kidney epithelium (Funder 1997); in the heart, MR signaling leads to hypertrophy and fibrosis. Therefore MR antagonists are commonly used for the treatment of hypertension and other cardiovascular diseases (Pitt et al. 1999). However, since GCs circulate in the organism at a much higher concentration than mineralocorticoids do, in cells devoid of $11 \beta$ - 
HSD II expression, e.g. macrophages and microglia, the MR is preferentially occupied by GCs rather than by aldosterone. This means that in myeloid cells both the GR and the MR are responsible for $\mathrm{GC}$ activities.

Importantly, the two receptors drive macrophage/microglia polarization in opposite directions. While GR-GC binding triggers a deactivating phenotype in macrophages (see Figure 1.2), GC binding to the MR has been associated with M1 macrophage polarization. Various experimental evidences support this hypothesis. Aldosterone treatment of peritoneal macrophages cultured in steroid-depleted medium resulted in increased expression of the pro-inflammatory markers TNF $\alpha$, MCP-1 or IL-12, effect that could be reverted by MR-blockade using eplerenone (Usher et al. 2010). Moreover, in vitro LPS stimulation of MR-deficient macrophages induced lower mRNA levels of M1 genes and higher expression of M2 markers compared to the wild type (wt) controls (Usher et al. 2010). In another study, MR activation by aldosterone in a microglial cell line also potentiated LPS induction of TNF $\alpha$ and IL-6, in line with the previous data. Additionally, it was shown that aldosterone binding to the MR activated NFKB, whereas the GR repressed it (Chantong et al. 2012). All in all, the balance between $M R$ and GR appears to play a relevant role in macrophage polarization. Whether this is also the case for other immune cells, like T cells or B cells, remains unclear and needs to be further investigated (Bene et al. 2014).

\subsubsection{Side effects of GC therapies}

A major drawback of GCs is the concurrence with adverse side effects (Moghadam-Kia and Werth 2010; Weinstein 2012; Ciriaco et al. 2013; Hunter et al. 2014a; Hwang and Weiss 2014). This problem derives from the ubiquitous expression of the glucocorticoid receptor (GR) (Rhen and Cidlowski 2005), that makes GCs important players in numerous homeostatic and metabolic processes. Although GC side effects in MS patients are rare due to the short duration of the treatment, in some clinical trials that tested pulsed long-term IVMP certain symptoms derived from the treatment were reported (Zivadinov et al. 2001). In fact, in other pathologies where regular GC application is the conventional therapy, this is a critical negative aspect that can even force the withdrawal of the treatment. 
One important side effect of GC therapy is hyperglycemia. This complication, defined as an abnormal increase in post-prandial and basal blood glucose, affects almost $50 \%$ of GCtreated patients and can lead to type II diabetes. GCs increase glucose levels via promotion of gluconeogenesis in the liver and reduction of glucose uptake in the skeletal muscle. They also affect pancreatic $\beta$ cells, diminishing insulin secretion, and can act as insulin antagonists leading to insulin resistance (Kuo et al. 2015). Another complication observed after GC therapy is skeletal muscle atrophy. Steroid myopathy is characterized by a reduction in the diameter of type II muscle fibers and lower myofibrillar protein content, due to a deregulation between protein synthesis and proteasomal degradation (Schakman et al. 2013; Braun and Marks 2015). Additionally, the occurrence of gastric ulcers in patients under GC therapy is also frequently reported. Recent studies demonstrated that dexamethasone (Dex) administration to mice leads to gastroparesis. This effect appears to be a result of a decreased production of NO, which is essential for gastric motility (Reichardt et al. 2014). As a consequence, stomach emptying is impaired and the weight of the filled stomach increases. Last but not least, the reduction in bone mineral density leading to osteoporosis is possibly the most severe GC-derived adverse symptom. Just a few weeks after low dose GCtreatment, the risk of bone fractures can increase up to $75 \%$, especially in post-menopausic women (Steinbuch et al. 2004). The GR is expressed in osteoblasts, osteocytes and osteoclasts, and can therefore alter bone production and re-sorption. However, studies using cell type-specific GR knock-out mice revealed that osteoblasts were the major targets of GCs in the context of osteoporosis, whereas the role of osteoclasts was of minor importance (Rauch et al. 2010). Among other effects, GCs diminish the production of new osteoblast progenitors interfering with the Wnt-signaling pathway, and induce premature osteoblast apoptosis, leading to a reduction of bone density.

Because of these and others clinical symptoms, GCs must be prescribed carefully, and the balance between the benefits and the disadvantages of the therapy must always be evaluated. 


\subsubsection{Emerging GC therapies: from dissociating ligands to nanoparticles}

As mentioned above, GCs are a mainstay for the treatment of MS as well as other inflammatory and autoimmune conditions, but their widespread effects are an important limiting factor for their clinical use. In recent years, big efforts have been made to improve the pharmacological features of GCs, and although many of the alternatives have shown good results in animal models, none of these formulations reached the market so far.

The results obtained from $G R^{\text {dim }}$ studies pointed to the trans-activation mechanism of the GR as the responsible mechanism for some of the metabolic adverse effects following GC treatment. Thus, taking advantage of the dual molecular pathway employed by the GR, different dissociating ligands were designed to act predominantly via the GR transrepression mechanism, among them, AL-438 (Coghlan et al. 2003) or Compound A (CpdA) (Louw et al. 1997). CpdA was shown to be effective in the treatment of EAE, however, its neurotoxic effects at high concentrations discarded it as a suitable drug for patients (Wüst et al. 2009).

In the design of drug delivery systems for the treatment of MS, the CNS compartment poses an additional challenge, since the formulation must be able to cross the BBB. This obstacle can be circumvented using biocompatible micro-/nanoparticles such as liposomes. Liposomes do not only cross the BBB due to their lipophilic character, but also have the right size to be taken up by phagocytic immune cells, such as macrophages and DCs, that will carry the drug to the CNS achieving a local therapeutic effect. The safety and tolerability of liposome-encapsulated drugs have been successfully evaluated in several clinical trials (Gordon et al. 2001; Alberts et al. 2004). In fact, liposomal doxorubicin is currently approved for the treatment of certain cancers and seems to overcome the signs of cardiopathy associated with the free drug (Tahover et al. 2015). Regarding GCs, some studies confirmed the therapeutic potential of liposomal corticosteroids in animal models of autoimmune inflammatory disorders, such as arthritis and MS (Metselaar et al. 2003; Linker et al. 2008; Schweingruber et al. 2011). Furthermore, liposomal encapsulation of GCs was shown to significantly increase the potency of the drug in the EAE mouse model (Schweingruber et al. 2011). 
As an alternative delivery system, current research focuses on the development of state of the art biocompatible nanoparticles with sizes ranging from 10 to $100 \mathrm{~nm}$. Due to this feature, nanoparticles are preferentially pinocytosed by inflammatory monocytes and macrophages, allowing the specific targeting of these immune cells. Larger particles, on the contrary, accumulate in the lungs and the liver and are phagocytosed mainly by peripheral DCs (Weissleder et al. 2014). Nanoparticles can be obtained from different organic materials, such as poly(lactic-co-glycolic acid), chytosan, alginate or silica, and can be additionally coated with antibodies (Abs) or other targeting molecules (Tabansky et al. 2015). Recently, a new modality of inorganic-organic hybrid nanoparticles (IOH-NPs) for the delivery of corticosteroids was developed (Heck et al. 2015). From a molecular point of view, IOH-NPs are composed of an inorganic bivalent cation reacting with equimolar amounts of an organic functional anion. In this case, betamethasone (BMZ) phosphate constitutes the organic part of the compound, and zirconium oxide $\left(\mathrm{ZrO}_{2}\right)$ was chosen as cation due to its stability and lack of physiological activity. In solution, this compound crystalizes in particles with a diameter of 40 to $90 \mathrm{~nm}$, and moreover, the combination with flavin mononucleotide (FMN) provides a fluorescent signal that allows their detection via flow cytometry or fluorescence microscopy. Preliminary in vitro experiments with these nanoparticles showed a good cell tolerability and proper delivery of the drug, however their therapeutic potential has not been evaluated in detail yet (Heck et al. 2015; Ring, unpublished data).

\subsection{Objectives}

T cells were for a long time believed to be major targets of the GC-therapy for acute relapses of MS (Wüst et al. 2008). However, myeloid cells like macrophages and microglia are affected by endogenous and therapeutic GCs as well, and their importance for the maintenance of tolerance and the pathogenesis of MS has gained attention over the years. Macrophage/microglia phenotypes are highly dynamic, and changes in their polarization state may determine the course of MS and EAE. In these myeloid cells, GCs can act either via the GR in a deactivating manner, or via the MR promoting a pro-inflammatory state (Usher et al. 2010), and this dual role of GCs makes the myeloid compartment particularly 
interesting in the context of neuroinflammation. In this doctoral thesis I aimed to define the roles of the $G R$ and the $M R$ in myeloid cells for the response to GCs during EAE. Furthermore, I propose that targeting GC therapy specifically to these immune cells might represent a strategy to improve the therapeutic features of the drug. To address these issues, two different approaches were employed:

- The first part of the project aimed to evaluate the in vitro and in vivo effects of BMZphosphate $\mathrm{ZrO}_{2}$ IOH-NPs (BNPs) (Heck et al. 2015). BNPs are expected to preferentially target myeloid cells due to their size, therefore their effects on different cell types and their in vivo cell specificity were analyzed. Furthermore, the therapeutic potential of the BNPs in the $M_{30 G}$-55-induced EAE mouse model and possible GC-derived side effects were studied.

- The second part of the project dealt with the role of the MR in myeloid cells in the context of CNS autoimmunity. Since MR-mediated responses counteract the immunosuppressive effect that GCs induce via the GR, the blockade of the MR might potentiate macrophage polarization towards a deactivated phenotype, thereby reducing CNS inflammation during EAE. To test this hypothesis, mice harboring a myeloid-specific MR deletion were studied after disease induction, and the different cellular events involved in disease initiation and progression were investigated. 


\section{MATERIAL AND METHODS}

\subsection{Material}

\subsubsection{General equipment}

Table 1.1 | General equipment

\begin{tabular}{|c|c|c|}
\hline Instrument & Model & Manufacturer \\
\hline Blood glucose meter & Ascensia CONTOUR & Bayer, Leverkusen \\
\hline \multirow[t]{4}{*}{ Centrifuges } & 5417R for reaction tubes & Eppendorf, Hamburg \\
\hline & 5804 for FACS tubes & Eppendorf, Hamburg \\
\hline & Multifuge $4 \mathrm{KR}$ for Falcon tubes & Heraeus, Hanau \\
\hline & Sigma $2-5$ for 96 -well plates & $\begin{array}{l}\text { Sigma Laborzentrifugen } \mathrm{GmbH} \text {, } \\
\text { Osterode am Harz }\end{array}$ \\
\hline Dehydration system & TP1020 & Leica Microsystems, Nussloch \\
\hline Electrophoresis chamber & & Peqlab Biotechnology, Erlangen \\
\hline $\begin{array}{l}\text { Electrophoresis power } \\
\text { supply }\end{array}$ & EPS 301 & Amersham Biosciences, Freiburg \\
\hline \multirow[t]{2}{*}{ FACS machines } & BD FACS Canto II & $\begin{array}{l}\text { Beckton Dickinson Biosciences, } \\
\text { Heidelberg }\end{array}$ \\
\hline & BD FACS Sorter FACSAria & $\begin{array}{l}\text { Beckton Dickinson Biosciences, } \\
\text { Heidelberg }\end{array}$ \\
\hline Gel imager & Chemostar & Intas $\mathrm{GmbH}$, Goettingen \\
\hline Incubator & HERACell 240 & Heraeus, Hanau \\
\hline Infrared Lamp & Balance 100W & Philips, Amsterdam, Netherlands \\
\hline \multirow[t]{2}{*}{ Laminar airflow cabinet } & HERASafe & Heraeus, Hanau \\
\hline & Interactive Safe Change station & $\begin{array}{l}\text { Tecniplast GmbH, } \\
\text { Hohenpeissenberg }\end{array}$ \\
\hline MACS & AutoMACS & $\begin{array}{l}\text { Miltenyi Biotech, Bergisch } \\
\text { Gladbach }\end{array}$ \\
\hline
\end{tabular}




\begin{tabular}{|c|c|c|}
\hline Micropipettes & $2.5,20,200$ and $1000 \mu \mathrm{L}$ & $\begin{array}{l}\text { Gilson, Middleton, Wisconsin, } \\
\text { USA }\end{array}$ \\
\hline \multirow[t]{3}{*}{ Microscopes } & Primo Star & Zeiss, Jena \\
\hline & Telaval 31 & Zeiss, Jena \\
\hline & Olympus BX51 & Olympus, Tokio, Japan \\
\hline Microtome & Leica SM2000R & Leica Microsystems, Nussloch \\
\hline Microwave oven & $\mathrm{R}-212$ & Sharp, Osaka, Japan \\
\hline $\begin{array}{l}\text { Neubauer improved } \\
\text { haemacytometer }\end{array}$ & & $\begin{array}{l}\text { Henneberg-Sander GmbH, } \\
\text { Giessen }\end{array}$ \\
\hline Ph-meter & 766 Calimatic & $\begin{array}{l}\text { Knick Elektronische Messegeräte } \\
\mathrm{GmbH} \text {, Berlin }\end{array}$ \\
\hline \multirow[t]{2}{*}{ Photometers } & Nanodrop 2000 & ThermoScientific, Erlangen \\
\hline & $\begin{array}{l}\text { BioTek }{ }^{\circledR} \text { Power Wave } 340 \text { plate } \\
\text { reader }\end{array}$ & BioTek, Bad Friedrichshall \\
\hline Pipette controller & Accu-jet ${ }^{\circledR}$ pro & Brand GmbH, Wertheim \\
\hline \multirow[t]{3}{*}{ Refrigerators } & Freezer Hera $-80^{\circ} \mathrm{C}$ & Heraeus, Hanau \\
\hline & Freezer Liebherr Comfort $-20^{\circ} \mathrm{C}$ & $\begin{array}{l}\text { Liebherr International } \mathrm{GmbH} \text {, } \\
\text { Biberach an der Riss }\end{array}$ \\
\hline & Freezer VIP plus $-150^{\circ} \mathrm{C}$ & $\begin{array}{l}\text { Sanyo Electric Co. Moriguchi, } \\
\text { Osaka }\end{array}$ \\
\hline Scale & TE313S & Sartorius AG, Göttingen \\
\hline Shaker & 3006 & $\begin{array}{l}\text { Gesellschaft fuer Labortechnik, } \\
\text { Burgwedel }\end{array}$ \\
\hline $\begin{array}{l}\text { Tuberculin glass/metal } \\
\text { syringes }(1,2 \mathrm{ml})\end{array}$ & & Hartenstein \\
\hline \multirow[t]{3}{*}{ Thermocyclers } & Thermocycler & Heraeus, Hanau \\
\hline & 7500 Real-Time PCR & $\begin{array}{l}\text { AB Applied Biosciences, Applera } \\
\text { GmbH, Darmstadt }\end{array}$ \\
\hline & Thermomixer Comfort & Eppendorf, Hamburg \\
\hline Tissue Embeding System & EG1160 & Leica Microsystems, Nussloch \\
\hline $\begin{array}{l}\text { UV system camera and gel } \\
\text { imager }\end{array}$ & & Intas GmbH, Göttingen \\
\hline Vortex mixer & Vortex Genie2 & Bohemia , NY, USA \\
\hline Water bath & W12 & Labortechnik Medigen, Dresde \\
\hline
\end{tabular}




\section{Water purification system \\ Arium $^{\circledR} 611$ \\ Sartorius AG, Göttingen \\ Millipore purification system \\ Millipore GmbH, Schwalbach}

\subsubsection{Consumables}

Table 1.2 |Consumables

\begin{tabular}{|c|c|c|}
\hline Product description & & Manufacturer \\
\hline 96-well Optical Reaction Plates & & $\begin{array}{l}\text { Applied Biosystems, Foster City, } \\
\text { California, USA }\end{array}$ \\
\hline Gavage cannula & & Cadence Science, Cranston, USA \\
\hline \multirow[t]{3}{*}{ Cell Culture Plates } & $4 \mathrm{~cm}, 10 \mathrm{~cm}$ & Sarstedt, Nümbrecht \\
\hline & $\begin{array}{l}\text { 6-well, } 24 \text {-well, } 48 \text {-well, } \\
\text { 96-well flat bottom }\end{array}$ & $\begin{array}{l}\text { Greiner bio-one } \mathrm{GmbH} \text {, } \\
\text { Frickenhausen }\end{array}$ \\
\hline & 96-well round bottom & $\begin{array}{l}\text { Greiner bio-one } \mathrm{GmbH} \text {, } \\
\text { Frickenhausen }\end{array}$ \\
\hline Cell strainer & $20 \mu \mathrm{m}, 40 \mu \mathrm{m}$ & BD Biosciences, Heidelberg \\
\hline ELISA plates & $\begin{array}{l}\text { Nunc Maxisorb flat } \\
\text { bottom } 96 \text { well plate }\end{array}$ & eBioscience, San Diego, USA \\
\hline FACS tubes & & BD Biosciences, Heidelberg \\
\hline Microscope slides & SuperFrost Plus & Menzel Glaeser, Braunschweig \\
\hline Needles & $\begin{array}{l}24 G 1^{\prime \prime}, 20 G 1 \frac{11}{\prime \prime} \\
27 G 3 / 4^{\prime \prime}, 25 G 1^{\prime \prime}\end{array}$ & B. Braun Melsungen AG, Melsungen \\
\hline Optical adhesive covers & & $\begin{array}{l}\text { Applied Biosystems, Foster City, } \\
\text { California, USA }\end{array}$ \\
\hline Pasteur pipettes & $3 \mathrm{~mL}$ & Th. Geyer GmbH, Renningen \\
\hline Pipettes & $\begin{array}{l}\text { Cellstar }^{\circledR} 5 \mathrm{~mL}, 10 \mathrm{~mL}, \\
25 \mathrm{~mL}\end{array}$ & $\begin{array}{l}\text { Greiner bio-one } \mathrm{GmbH} \text {, } \\
\text { Frickenhausen }\end{array}$ \\
\hline Pipette tips & $10 \mu \mathrm{L}, 200 \mu \mathrm{L}, 1000 \mu \mathrm{L}$ & $\begin{array}{l}\text { Greiner bio-one } \mathrm{GmbH} \text {, } \\
\text { Frickenhausen }\end{array}$ \\
\hline \multirow[t]{3}{*}{ Reaction tubes } & $0.5 \mathrm{~mL}$ & Sarstedt, Nümbrecht \\
\hline & $1.5 \mathrm{~mL}, 2 \mathrm{~mL}$ & $\begin{array}{l}\text { Greiner bio-one } \mathrm{GmbH} \text {, } \\
\text { Frickenhausen }\end{array}$ \\
\hline & $15 \mathrm{~mL}, 50 \mathrm{~mL}$ & $\begin{array}{l}\text { Greiner bio-one } \mathrm{GmbH} \text {, } \\
\text { Frickenhausen }\end{array}$ \\
\hline
\end{tabular}




\begin{tabular}{lll}
\hline Syringes & $1 \mathrm{~mL}$ & Henke Sass Wolf, Tuttlingen \\
& $2 \mathrm{~mL}, 5 \mathrm{~mL}, 60 \mathrm{~mL}$ & BD Biosciences, Heidelberg \\
Serum separation tubes & $\mathrm{STT}^{\mathrm{TM}}$ BD Microtainer ${ }^{\circledR}$ & BD Biosciences, Franklin Lakes, USA \\
Tissue cassettes & MacrOfFlow & Microm International, Waldorf \\
\hline
\end{tabular}

Unless otherwise indicated, all companies are located in Germany

\subsubsection{Chemicals and reagents}

Table 1.3 |Chemicals and reagents

\begin{tabular}{|c|c|}
\hline Chemical & Manufacturer \\
\hline 2- $\beta$-Mercaptoethanol & Invitrogen, Paisley, UK \\
\hline Acetic acid glacial $100 \%$ & Carl Roth, Karlsruhe \\
\hline Agarose UltraPure & Sigma-Aldrich, Taufkirchen \\
\hline Betamethasone phosphate (Celestan ${ }^{\circledR}$ ) & MSD Sharp and Dohme GmbH, Haar \\
\hline Bovine Serum Albumin (BSA) & Carl Roth, Karlsruhe \\
\hline $\mathrm{CaCl}_{2} \times 2 \mathrm{H}_{2} \mathrm{O}$ & Merk, Darmstadt \\
\hline Carboxyfluorescein succynimidyl ester (CFSE) & Life Technologies, Darmstadt \\
\hline Citric acid & Merk, Darmstadt \\
\hline Complete Freund's Adjuvant (CFA) & Difco Laboratories, Detroit, USA \\
\hline Dexa-ratiopharm ${ }^{\circledR} 100 \mathrm{mg}$ Injektionlösung & Ratiopharm GmbH, Ulm \\
\hline D-Glucose & Merk, Darmstadt \\
\hline 3,3'-Diaminobenzidine tetrahydroclhoride (DAB) & Sigma-Aldrich, Taufkirchen \\
\hline Dimethyl sulfoxide (DMSO) & Carl Roth, Karlsruhe \\
\hline DNA ladder $1 \mathrm{~kb}$ & Fermentas GmbH, St. Leon-Rot \\
\hline dNTPs & Genaxxon bioscience, Ulm \\
\hline
\end{tabular}




\begin{tabular}{|c|c|}
\hline Entellan & Merck, Darmstadt \\
\hline Ethanol $\geq 99,8 \%$ & Carl Roth, Karlsruhe \\
\hline Ethylendiaminetetraacetic acid (EDTA) & Sigma-Aldrich, Taufkirchen \\
\hline Ethidium Bromide & Carl Roth, Karlsruhe \\
\hline $10 \%$ stripped Fetal Calf Serum (FCS) & Invitrogen, Paisley, UK \\
\hline $\mathrm{H}_{2} \mathrm{O}_{2} 30 \%$ & Carl Roth, Karlsruhe \\
\hline $\mathrm{H}_{2} \mathrm{SO}_{4}$ & Merk, Darmstadt \\
\hline $\begin{array}{l}\text { 4-(2-hydroxyethyl)-1-piperacinethane-sulfonic acid } \\
\text { (HEPES) }\end{array}$ & Merk, Darmstadt \\
\hline iScript Reaction Mix & BIO-Rad, USA \\
\hline Ketamine $10 \%$ & MediStar, Aschenberg \\
\hline $\mathrm{KCl}$ & Merk, Darmstadt \\
\hline $\mathrm{KH}_{2} \mathrm{PO}_{4}$ & Merk, Darmstadt \\
\hline $\mathrm{Na}_{2} \mathrm{HPO}_{4} \times 12 \mathrm{H}_{2} \mathrm{O}$ & Merk, Darmstadt \\
\hline $\mathrm{Na}_{3} \mathrm{C}_{6} \mathrm{H}_{5} \mathrm{O}_{7}$ & Carl Roth, Karlsruhe \\
\hline $\mathrm{NaCl}$ & Carl Roth, Karlsruhe \\
\hline $\mathrm{Na}_{2} \mathrm{HPO}_{4} \times \mathrm{H}_{2} \mathrm{O}$ & Merk, Darmstadt \\
\hline $\mathrm{NaN}_{3}$ & Carl Roth, Karlsruhe \\
\hline $\begin{array}{l}\text { Myelin Oligodendrocyte Glycoprotein 35-55 } \\
\text { peptide (MOG } 35-55)\end{array}$ & Charité, Berlin \\
\hline Orange G & Sigma-Aldrich, Taufkirchen \\
\hline Optilyse ${ }^{\circledR}$ & Immunotech, Marseille, France \\
\hline Paraformaldehyde Histofix $4 \%$ & Carl Roth, Karlsruhe \\
\hline Penicillin/ Streptomycin & $\mathrm{GIBCO}^{\circledR}$ Invitrogen, Paisley, UK \\
\hline Percoll ${ }^{\circledast}$ & Sigma, St. Louise, USA \\
\hline Sodium dodecyl sulfate (SDS) & SERVA GmbH, Heidelberg \\
\hline 3,3',5,5'-Tetramethylbenzidin (TMB) & Sigma-Aldrich, Taufkirchen \\
\hline
\end{tabular}




\begin{tabular}{ll|}
\hline Thioglycolate & Sigma-Aldrich, Taufkirchen \\
Tris & Carl Roth, Karlsruhe \\
Trypan blue & Sigma Aldrich, Taufkirchen \\
Tween 20 & Carl Roth, Karlsruhe \\
Xylariem & (Xylazine 2\%) \\
Xylol & Riemser, Greifswald-Insel Riems \\
\hline
\end{tabular}

\subsubsection{Media, buffers and solutions}

Table 1.4 | Media

\begin{tabular}{lll} 
Medium & Composition or additives & Manufacturer \\
\hline DMEM+GlutaMAX ${ }^{\mathrm{TM}}$ & $+10 \%$ FCS & GIBCO $^{\circledR}$ Invitrogen, \\
& $+0.01 \%$ Penicillin/Streptomycin & Paisley, UK \\
RPMI Medium 1640 + GlutaMAX ${ }^{\mathrm{TM}}$ & $+10 \%$ FCS & GIBCO $^{\circledR}$ Invitrogen, \\
& $+0.01 \%$ Penicillin/Streptomycin & Paisley, UK \\
Re-stimulation medium (ReMed) & RPMI Medium 1640 & \\
& $+5 \%$ FCS & \\
& $+0.01 \%$ Penicillin/Streptomycin & Paisley, UK \\
& $+1 \%$ Sodium pyruvat & \\
& $+0.2 \% \beta$-mercaptoethanol & \\
\hline
\end{tabular}

Unless otherwise indicated, all companies are located in Germany 
Table 1.5 |Buffers and solutions

Buffer or solution

Alsevers

Annexin binding buffer

\begin{tabular}{|c|c|c|}
\hline Buffer or solution & Composition & \\
\hline \multirow[t]{4}{*}{ Alsevers } & $27 \mathrm{mM} \mathrm{NaCl}$ & \\
\hline & 125 mM D-Glucose & \\
\hline & $3 \mathrm{mM}$ Citric Acid & \\
\hline & $30 \mathrm{mM} \mathrm{Na}_{3} \mathrm{C}_{6} \mathrm{H}_{5} \mathrm{O}_{7}$ & in $d_{d d} \mathrm{H}_{2} \mathrm{O}$ \\
\hline \multirow[t]{3}{*}{ Annexin binding buffer } & $10 \mathrm{mM}$ HEPES/NaOH pH7.4 & \\
\hline & $140 \mathrm{mM} \mathrm{NaCl}$ & \\
\hline & $2.5 \mathrm{mM} \mathrm{CaCl}_{2}$ & in $\mathrm{ddH}_{2} \mathrm{O}$ \\
\hline Citrate buffer pH 6 & 109 mM Citric Acid & in $\mathrm{dd} \mathrm{H}_{2} \mathrm{O}$ \\
\hline ELISA Assay diluent & $10 \%$ FCS & in PBS \\
\hline ELISA Carbonate coating buffer pH 9.5 & $0.1 \mathrm{M} \mathrm{Na}_{2} \mathrm{CO}_{3}$ & in $\mathrm{ddH}_{2} \mathrm{O}$ \\
\hline ELISA Phosphate coating buffer pH 6.5 & $0.1 \mathrm{M} \mathrm{Na}_{2} \mathrm{HPO}_{4}$ & in $d_{d d H_{2}} \mathrm{O}$ \\
\hline \multirow[t]{2}{*}{ ELISA Substrate buffer } & 0.1M Citric Acid & \\
\hline & $0.2 \mathrm{M} \mathrm{Na}{ }_{2} \mathrm{HPO}_{4}$ & in $\mathrm{dd} \mathrm{H}_{2} \mathrm{O}$ \\
\hline \multirow[t]{3}{*}{ ELISA Developing solution } & Substrate buffer & \\
\hline & $1 \%$ TMB in DMSO & \\
\hline & $0.2 \% \mathrm{H}_{2} \mathrm{O}_{2}$ & \\
\hline \multirow[t]{2}{*}{ FACS buffer } & $0.1 \% \mathrm{BSA}$ & \\
\hline & $0.01 \% \mathrm{NaN}_{3}$ & in $\mathrm{PBS}$ \\
\hline \multirow[t]{2}{*}{ MACS buffer } & $0.5 \%$ BSA & \\
\hline & $2 \mathrm{mM}$ EDTA & in PBS \\
\hline \multirow[t]{4}{*}{ Phosphate Saline Buffer pH 7.4 (PBS) } & $137 \mathrm{mM} \mathrm{NaCl}$ & \\
\hline & $2.7 \mathrm{mM} \mathrm{KCl}$ & \\
\hline & $10 \mu \mathrm{M} \mathrm{Na}{ }_{2} \mathrm{HPO}_{4}$ & \\
\hline & $2.0 \mathrm{mM} \mathrm{KH}_{2} \mathrm{PO}_{4}$ & in $\mathrm{dd}_{2} \mathrm{O}$ \\
\hline
\end{tabular}

\section{Composition}

$27 \mathrm{mM} \mathrm{NaCl}$

125 mM D-Glucose

3 mM Citric Acid

$30 \mathrm{mM} \mathrm{Na}_{3} \mathrm{C}_{6} \mathrm{H}_{5} \mathrm{O}_{7}$

in $\mathrm{ddH}_{2} \mathrm{O}$

10 mM HEPES/NaOH pH7.4

$140 \mathrm{mM} \mathrm{NaC}$ 


\begin{tabular}{|c|c|c|}
\hline PBS/BSA & $0.1 \% \mathrm{BSA}$ & in PBS \\
\hline PBS/Tween & $0.1 \%$ Tween 20 & in PBS \\
\hline \multirow[t]{2}{*}{ Percoll diluent } & $0.1 \% \mathrm{BSA}$ & \\
\hline & $1 \%$ Glucose & in PBS \\
\hline \multirow[t]{3}{*}{ Spinal cord suspension buffer } & $0.1 \% \mathrm{BSA}$ & \\
\hline & $1 \%$ Glucose & \\
\hline & $100 \mu \mathrm{g} / \mathrm{mL}$ DNase & in PBS \\
\hline \multirow[t]{2}{*}{ Sulfite wash } & $1 \% \mathrm{HCl}$ & \\
\hline & $0.4 \% \mathrm{~K}_{2} \mathrm{~S}_{2} \mathrm{O}_{5}$ & in $\mathrm{dd}_{2} \mathrm{O}$ \\
\hline \multirow[t]{2}{*}{ TAC buffer pH 7.2} & $155 \mathrm{mM} \mathrm{NH}_{4} \mathrm{Cl}$ & \\
\hline & $20 \mathrm{mM}$ Tris & in $\mathrm{ddH}_{2} \mathrm{O}$ \\
\hline \multirow[t]{4}{*}{ Tail buffer } & $1 \%$ SDS & \\
\hline & $100 \mathrm{mM} \mathrm{NaCl}$ & \\
\hline & 100 mM EDTA & \\
\hline & $5 \mathrm{mM}$ Tris & in $\mathrm{ddH}_{2} \mathrm{O}$ \\
\hline
\end{tabular}

\subsubsection{Enzymes and commercial kits}

Table 1.6 |Enzymes and commercial kits

\begin{tabular}{ll|} 
Product & Manufacturer \\
\hline Cytometric Bead Array (CBA) & BD Bioscience \\
Mouse Th1, Th2, Th17 cytokine kit & \\
Easy Sep ${ }^{\mathrm{TM}}$ Mouse T cell Isolation Kit & StemCell ${ }^{\mathrm{TM}}$ Technologies \\
Intracellular FACS Staining Kit & eBioscience, San Diego USA \\
iScript cDNA Synthesis Kit & Bio-Rad Laboratories, Munich \\
Mouse IFNy ELISA MAX & BioLegend, San Diego, USA \\
\hline
\end{tabular}


Mouse TNF $\alpha$ ELISA MAX ${ }^{\text {TM }}$ Standard Set

Mouse IL-17A ELISA MAX'M Standard Set

$\begin{array}{ll}\text { Mouse Insuline ELISA } & \text { RayBiotech Inc., Heidelberg } \\ \text { Osteocalcin ELISA } & \text { R\&D Systems, USA } \\ \text { Mouse Renin ELISA } & \text { RayBiotech Inc., Heidelberg } \\ \text { Mouse GM-CSF ELISA reagents } & \text { R\&D Systems, USA } \\ \text { Mouse IL-17A ELISA reagents } & \text { Miltenyi Biotech, Bergisch Gladbach } \\ \text { Pan T cell isolation Kit II, Mouse } & \text { Thermo Scientific, Waltham, USA } \\ \text { Phusion }{ }^{\circledR} \text { DNA Polymerase and 5x Reaction } & \\ \text { Buffer HF } & \text { Applied Biosystems, Foster City, USA } \\ \text { Power SYBR }{ }^{\circledR} \text { Green PCR Master Mix } & \text { Qiagen, Hilden } \\ \text { RNeasy Mini Kit } & \text { AppliChem GmbH, Darmstadt } \\ \text { Proteinase K } & \text { Zymo Research, Irvine, USA } \\ \text { Quick-RNA MiniPrep } & \end{array}$

\subsubsection{FACS Antibodies}

Table 1.7 |Antibodies for flow cytometry

\begin{tabular}{|c|c|c|c|c|}
\hline Specificity & Clone & Fluorochrome & Isotype & Manufacturer \\
\hline $\mathrm{CD} 3 \varepsilon$ & $17 A 2$ & $\begin{array}{l}\text { PerCP } \\
\text { APC }\end{array}$ & Rat IgG2b, $\mathrm{k}$ & BioLegend \\
\hline CD4 & RM4-5 & $\begin{array}{l}\text { PerCP } \\
\text { APC-Cy7 }\end{array}$ & Rat IgG2a, к & BD Biosciences \\
\hline $\operatorname{CD} 8 \alpha$ & $53-6.7$ & $\mathrm{PE}$ & Rat IgG2a, к & BioLegend \\
\hline CD11b & $\mathrm{M} 1 / 70$ & PE-Cy7 & Rat IgG2b, $k$ & BioLegend \\
\hline
\end{tabular}




\begin{tabular}{|c|c|c|c|c|}
\hline CD11c & N418 & bio & $\begin{array}{l}\text { Armenian } \\
\text { Hamster IgG }\end{array}$ & BD Biosciences \\
\hline CD25 & PC61 & APC-Cy7 & Rat $\lg G 1, \lambda$ & BioLegend \\
\hline CD45.2 & 104 & APC & Mouse IgG2a, k & BD Biosciences \\
\hline CD45R/B220 & RA3-6B2 & PE & Rat IgG2a, k & BD Biosciences \\
\hline CD86 & GL-1 & PE & Rat IgG2a, k & BioLegend \\
\hline CD206 & $\mathrm{C} 068 \mathrm{C} 2$ & APC & Rat IgG2a, k & BioLegend \\
\hline Ly6C & HK1.4 & FITC & Rat $\lg G 2 \mathrm{c}, \mathrm{K}$ & BD Biosciences \\
\hline Ly6G & $1 \mathrm{~A} 8$ & PE & Rat IgG2a, $\mathrm{k}$ & BD Biosciences \\
\hline I-A $(M H C-I I)$ & AF6-120.1 & PE & $\begin{array}{l}\text { Mouse (BALB/c) } \\
\operatorname{lgG} 2 \mathrm{a}, \mathrm{k}\end{array}$ & BioLegend \\
\hline FoxP3 & FJK-16s & APC & FJK-16s & eBioscience \\
\hline Annexin V & & Cy5 & & BD Biosciences \\
\hline CD16/CD32 (Fc-block) & $2.4 \mathrm{G} 2$ & & Rat IgG2a, $\lambda$ & BioLegend \\
\hline
\end{tabular}

\subsubsection{IHC Antibodies}

Table 1.8 |Antibodies for immunohistochemistry

\begin{tabular}{llll} 
Specificity & Clone & Isotype & Manufacturer \\
\hline Human CD3 & CD3-12 & Rat IgG1 & AbD Serotec $^{\circledR}$ \\
Mouse MAC3 & M3/84 & Rat IgG1 & BD Pharmigen ${ }^{\circledR}$ \\
Rat IgG (biotinilated) & & Rabbit & Vector Laboratories \\
\hline
\end{tabular}




\subsubsection{Oligonucleotides}

Table 1.9 | Primers for qRT-PCR

\begin{tabular}{lll} 
Target gene & & Sequence (5' - 3') \\
\hline \multirow{2}{*}{ mHP-1 } & fwd & GCC CAA GAT GGA CGC AAT C \\
& rev & CCG AGG CGC CAG TCT TC \\
mFKBP51 & fwd & GAA CCT GGC CAT GTG CTA CCT \\
& rev & GTC CAG TCC AAG GGC CTT GT \\
mPEPCK & fwd & AAA GCA TTC AAC GCC AGG TT \\
& rev & TGC TGA ATG GGA TGA CAT ACA TG \\
mTAT & $f w d$ & CCT CTG GAA GCT AAG GAT GTC ATT \\
& rev & AAC ACG GCT AGA CAC AGC TCA A \\
\hline
\end{tabular}

\subsubsection{Software}

Table 1.10 |Software

\begin{tabular}{ll}
\hline Software & Developer \\
\hline analySIS $^{\text {B }}$ & Olympus, Tokio, Japan \\
\hline BD FACSDiva Software v6.1.2 & BD Biosciences, Heidelberg \\
\hline FCAP Array Software v3.0.1 & BD Biosciences, Heidelberg \\
\hline FlowJo Software v 7.6 & Tree Star, Inc., Ashland, Oregon, USA \\
\hline Gen5 v1.09.8 & BioTek Instruments, Bad Friedrichshall \\
GraphPad Prism for Windows v5.04 & GraphPad Software, La Jolla, CA, USA \\
\hline ImageJ 1.46r & Wayne Rasband Nat. Inst. Of Health, USA \\
Intas GDS & Intas, Göttingen \\
\hline
\end{tabular}

Unless otherwise indicated, all companies are located in Germany 


\subsection{Animal experimentation}

All the mice used during this project had a C57BL/6 background and were either purchased from Charles River Laboratories (Sulzfeld, Germany) or bred in the breeding facilities of the University Medical Center in Göttingen. The animals were housed in individually ventilated cages (IVC) under specific pathogen-free conditions (SFP) with 12 hours day/night cycle, and water and food were given ad libitum. The mice used for experimentation were at least 10 weeks old. All experiments were approved by the responsible authorities of Lower Saxony and conducted in accordance to the ethical standards of humane animal care.

\subsubsection{Mouse strains}

○ C57BL/6: wt, background strain (Charles River Laboratories, Wilmington, USA).

$\circ$ GR $^{\mathrm{fl}}: \mathrm{Nr3c1^{ \text {tm2Gsc } }}$ (Tronche et al. 1999). This mouse strain contains loxP sites flanking exon 3 of the nuclear receptor subfamily 3 , group $\mathrm{C}$, member 1 (Nr3c1) gene, coding for the GR.

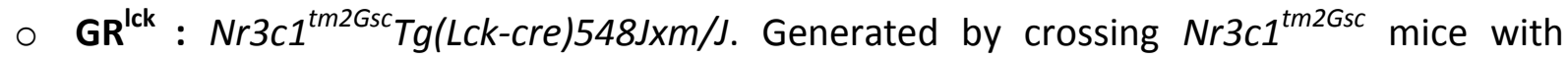
$T g($ Lck-cre)548Jxm/J mice (Baumann et al. 2005). These mice express the Cre recombinase under the control of the lymphocyte protein tyrosine kinase (Lck) promoter, which mediates T cell-specific excision of the GR sequence.

○ $\mathbf{G R}^{\text {LysM }}: N r 3 c 1^{\text {tm2Gsc }}$ Lyz2tm1(cre)lfo/J. Generated by crossing $N r 3 c 1^{\text {tm2Gsc }}$ mice with Lyz2tm1(cre)lfo/J mice (Clausen et al. 1999). In these mice, the Cre recombinase is expressed under the control of the lysozyme 2 gene promoter (lyz2), mediating the targeted deletion of the GR in monocytes, mature macrophages and granulocytes. 
○ $\mathbf{G R}^{\text {Lck/LysM }}: N r 3 c 1^{\text {tm2Gsc }} \mathrm{Tg}($ Lck-cre)548Jxm/J Lyz2tm1(cre)lfo/J. This mouse strain expresses the Cre recombinase under both the Lck and the Lyz2 promoters, mediating the excision of the GR in T cells and cells from the myeloid lineage.

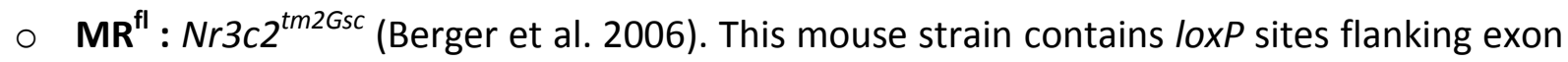
3 of the nuclear receptor subfamily 3 , group $\mathrm{C}$, member $2(\mathrm{Nr} 3 \mathrm{c} 2)$ gene, coding for the MR.

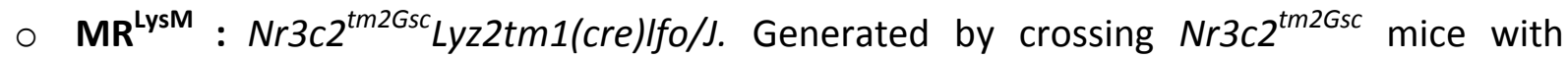
Lyz2tm1(cre)lfo/J mice (Rickard et al. 2009). In these mice, the Cre recombinase is expressed under the control of the lysozyme 2 gene promoter (lyz2), mediating the targeted deletion of the MR in monocytes, mature macrophages and granulocytes.

○ GR $^{\text {SLCO1C1 }}: N r 3 c^{1 t m 2 G s c} T g(S / c o 1 c 1-i c r e / E R T 2) 1 M r k s$. Generated by crossing Nr3c1 ${ }^{\text {tm2Gsc }}$ mice with Tg(S/co1c1-icre/ERT2)1Mrks mice (Ridder et al. 2011). These mice carry a tamoxifeninducible gene to express the Cre recombinase in brain endothelial cells, but not in other endothelial cells, mediating the deletion of the GR in this cell type.

- 2D2 RFP: (Tcra2D2,Tcrb2D2)1Kuch/J (Bettelli et al. 2003). Transgenic mouse strain expressing a fully competent, $\mathrm{MOG}_{35-55}$ specific TCR. This strain was back-crossed with RFP mice (Gt(ROSA)26Sor ${ }^{\text {tm1 } 1 \mathrm{Hif}}$, Luche et al. 2007), expressing the red-fluorescent protein to confer red fluorescence to the carrying cells.

\subsubsection{Mouse genotyping}

In order to assess the genotype of the newborns from our inbred transgenic strains, tail biopsies were collected four weeks after birth and DNA was isolated and used for PCR with the correspondent primers (see 2.7.1). Generally, Cre negative animals were used as a control to their respective knock-out for our experiments. 


\subsubsection{Tamoxifen-induction of SLO1C1 knockout mice}

The deletion of the GR from endothelial cells of the BBB was achieved via tamoxifen treatment 4 weeks prior to starting the experiment. Tamoxifen was dissolved in sunflower oil and left overnight $(\mathrm{O} / \mathrm{N})$ at $37^{\circ} \mathrm{C}$. Three consecutive doses of $3 \mathrm{mg}$ of tamoxifen were administered every other day via oral gavage with a bulb-tipped gastric gavage needle.

\subsubsection{Anesthesia}

Mice subjected to EAE experiments were anesthetized before subcutaneous injection of the immunizing agent. A preparation of $1 \%$ Ketamine and $0.01 \%$ Xylazine $\left(X\right.$ ylariem $\left.{ }^{\circledR}\right)$ diluted in $0.9 \% \mathrm{NaCl}$ solution was injected i.p. at a dose of $10 \mu \mathrm{l}$ per gram of body weight. Mice under anesthesia were kept warm to prevent hypothermia and a moistening balm was applied over the eyes to maintain hydration.

\subsubsection{Blood sample processing}

Blood was obtained either from the tail of living mice, or via heart puncture on $\mathrm{CO}_{2^{-}}$ sacrificed animals when higher blood volumes were needed.

Blood samples analyzed by FACS were collected directly on Alsevers, centrifuged 5 min at $350 \times \mathrm{g}$, and stained for surface markers with the correspondent fluorescent-labeled antibodies (Abs). After washing with FACS buffer (350 x g, $5 \mathrm{~min}$ ), cells were incubated with

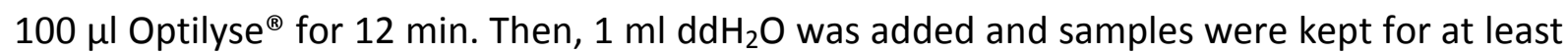
$2 \mathrm{~h}$ in the dark before they could be analyzed in the FACS device.

For serum extraction, at least $200 \mu \mathrm{l}$ blood obtained by heart puncture were left to clot in a Microtainer ${ }^{\mathrm{TM}}$ serum collection tube for $20 \mathrm{~min}$. Then tubes were centrifuged at $350 \mathrm{xg}$ for 2 min, and serum was removed with the help of a pipette. Serum samples were stored at $20^{\circ} \mathrm{C}$. 


\subsection{In vivo assays}

\subsubsection{EAE induction}

For all the experiments described here, EAE was induced by active immunization with $50 \mu \mathrm{g}$ $\mathrm{MOG}_{35-55}$ peptide. First, an emulsion of $1 \mathrm{mg} / \mathrm{ml} \mathrm{MOG}_{35-55}$ in $1 \mathrm{mg} / \mathrm{ml}$ CFA at a 1:1 ratio was prepared and left for $1 \mathrm{~h}$ at $4^{\circ} \mathrm{C}$. Once the mice were anesthetized, $50 \mu \mathrm{l}$ of the antigencontaining emulsion were injected s.c. in each flank at the beginning of the tail. Additionally, $200 \mathrm{ng}$ of Pertussis toxin diluted in $200 \mu \mathrm{l}$ of a $0,9 \% \mathrm{NaCl}$ solution were i.p. injected to help disrupting the BBB and boost immunization. Two days later, a second dose of Pertussis toxin was applied (Figure 2.1).

From day 9 on following immunization, mice were weighted daily and disease progression was monitored. EAE symptoms were evaluated according to the following 0 to 10 scoring scale: $0=$ Healthy; $1=$ Reduced tone of the tail; $2=$ Total paralysis of the tail; $3=$ Gait disturbance; 4= Gait ataxia in hind limbs; $5=$ Mild paresis of the hind limbs; $6=$ Moderate paraparesis; $7=$ Severe paraparesis or paraplegia; $8=$ Tetraparesis; $9=$ Moribund; $10=$ Death (Linker et al. 2008). Due to ethical reasons, mice with score 6 or higher were provided with special bedding and wet food, and mice reaching a score over 7 were sacrificed.

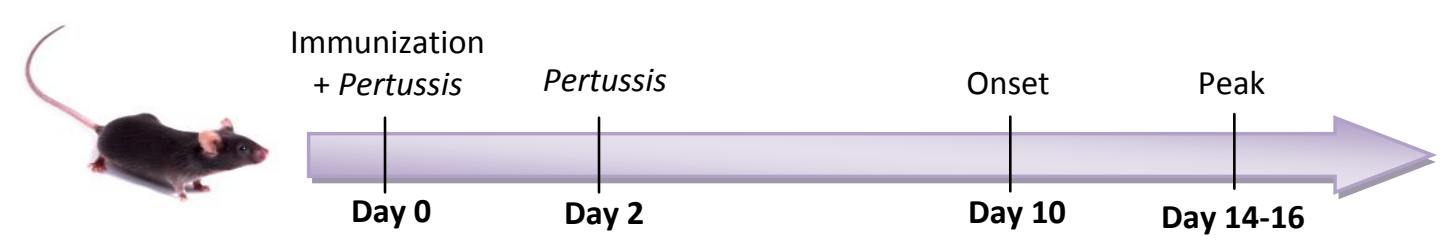

Figure 2.1 | EAE induction protocol. The timeline for EAE induction in C57BL/6 mice considers the day of immunization as day 0. Pertussis toxin is given as an adjuvant on days 0 and 2 after immunization, and the first symptoms of the disease typically appear from day 9 on, reaching the peak of disease around day 14-16. 


\subsubsection{In vivo $\mathrm{T}$ cell priming and proliferation}

To test the ability of MR ${ }^{\mathrm{lysM}} \mathrm{APCs}$ to prime $\mathrm{T}$ cells and promote their proliferation during the early phases of EAE the following protocol was carried out. T cells were purified from lymphoid organs of RFP 2D2 mice and labeled with CFSE as described below (see 2.4.5). $1 \cdot 10^{6}$ CFSE-labeled RFP 2D2 T cells were then i.v. injected into either $\mathrm{MR}^{\mathrm{fl}}$ (control) or MR $\mathrm{R}^{\mathrm{lysM}}$ mice. Two days later, mice were immunized according to our standard protocol (see 2.3.1). At day 3 and 5 after immunization the mice were sacrificed and lymphoid organs were isolated to analyze the proliferation cycles of the previously injected $T$ cells via flow cytometric analysis of the $\mathrm{RFP}^{+} \mathrm{CSFE}^{+}$cells.

\subsubsection{GC treatment}

Mice were injected i.p. daily on three consecutive days with $10 \mathrm{mg} / \mathrm{kg}$ of Dex, a corticosteroid 25 more potent than cortisol with minimal mineralocorticoid activity. In exceptional cases, BMZ was used at the same dose parallel to Dex. The equivalent volume of PBS was injected as a vehicle control.

One of the aims of this thesis work was to evaluate a novel corticoid drug where the corticosteroid, in this case $\mathrm{BMZ}$, is delivered by an inorganic compound $\left(\mathrm{ZrO}_{2}\right)$ in the form of nanoparticles (BNPs). To test the efficacy of BNPs, $100 \mu$ of the nanoparticle suspension, equivalent to $10 \mathrm{mg} / \mathrm{kg}$ of $\mathrm{BMZ}$, were injected i.p. during either one or three consecutive days. The same volume of nanoparticles without betamethasone (ENP) was used as a control.

When applied therapeutically on animals with EAE, the treatment was started once the animals showed the first symptoms (score $\geq 1$ ). 


\subsubsection{In vivo $\mathrm{T}$ cell apoptosis}

The potential of the BNPs to induce $\mathrm{T}$ cell apoptosis in vivo was evaluated treating wt B57BL/6 mice for one and three days with PBS, Dex, ENPs and BNPs. One day after the last injection, spleens were removed and total splenocyte numbers were determined under the microscope. Additionally, cells were stained with fluorophore-coupled Abs against CD3, CD4 and CD8 and analyzed via FACS (Figure 2.2).
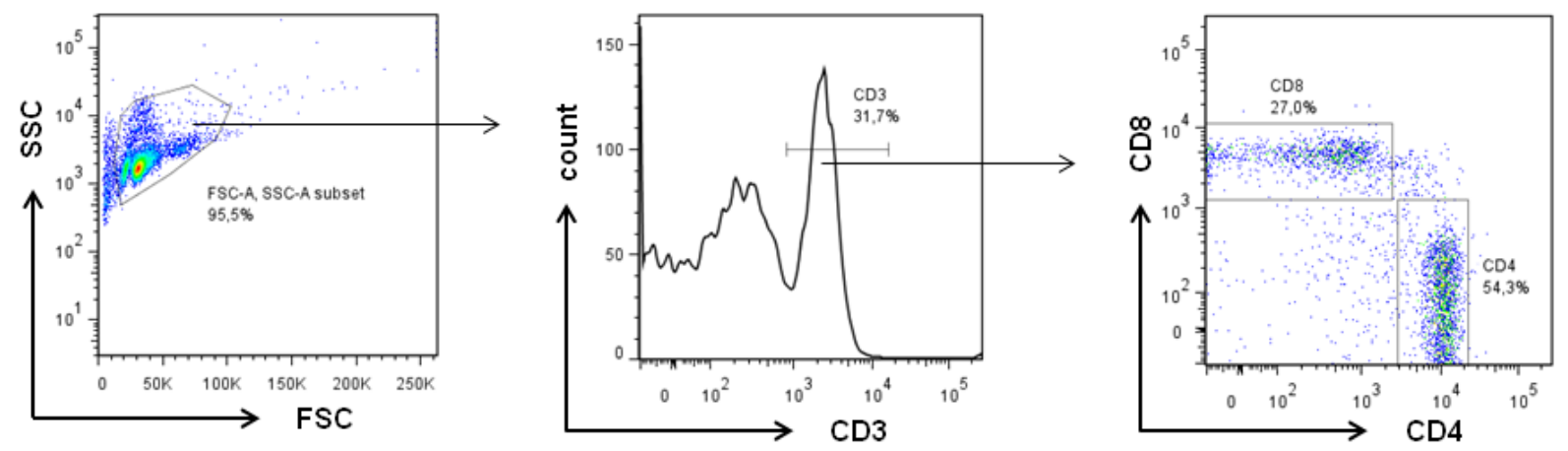

Figure 2.2 | FACS gating strategy for different T cell subsets. First, living cells were identified in the FSC/SSC panel. T cells were defined as $\mathrm{CD}^{+}$cells, and within this population Th cells could be distinguished from cytotoxic $T$ cells by the expression of the co-receptors $C D 4$ and $C D 8$, respectively.

\subsubsection{Analysis of glucose metabolism}

To analyze the effects of Dex and BNPs in glucose release and metabolism, mice were treated with PBS, $10 \mathrm{mg} / \mathrm{kg}$ Dex, ENPs and $10 \mathrm{mg} / \mathrm{kg}$ BNPs i.p. on four consecutive days. Following the last treatment, food pellets were removed O/N. Fasting blood sugar levels were determined in the morning with an Ascensia CONTOUR glucose-meter in blood droplets obtained via tail puncture.

Following glucose measurement, mice received an additional dose of the correspondent drug; $2.5 \mathrm{~h}$ later mice were sacrificed and plasma, serum and liver samples were obtained 
and snap frozen. Circulating insulin levels were analyzed via ELISA, and expression of gluconeogenetic enzymes in the liver via qRT-PCR

\subsection{Cellular methods}

\subsubsection{Cell isolation from secondary lymphoid organs}

Mice were sacrificed via $\mathrm{CO}_{2}$ inhalation. Afterward, spleens and cervical, axillary, mesenteric, inguinal and lumbar lymph nodes were removed and placed on a Petri dish with PBS/BSA. Single cell suspensions were obtained by homogenizing the tissue with the help of a syringe plunger and passing it through a $40 \mu \mathrm{m}$ cell strainer to avoid cell clumps. Cells were washed in PBS/BSA and centrifuged at $300 \times \mathrm{g}, 4^{\circ} \mathrm{C}$ for 7 minutes. In the case of splenocytes, erythrocytes were additionally lysed by incubation with $6 \mathrm{ml} \mathrm{TAC}$ buffer/ml of cell suspension for $12 \mathrm{~min}$, followed by centrifugation at $300 \times \mathrm{g}, 4^{\circ} \mathrm{C}$ for $7 \mathrm{~min}$. Pellets were resuspended in 1-2 ml PBS/BSA or the corresponding buffer. Subsequently, $10 \mu \mathrm{L}$ of the cell suspension were diluted in a Trypan-blue solution and living cell numbers were determined under the microscope using a Neubauer haemocytometer.

\subsubsection{Mononuclear cell isolation from the spinal cord}

Mice were euthanized with $\mathrm{CO}_{2}$ and perfused with $20 \mathrm{ml}$ of saline solution to clean vessels from blood. Then, the spine of the mice was removed and vertebrae were cut open in order to obtain the spinal cords, which were homogenized with a syringe plunger in spinal cord suspension buffer. The suspension was washed and centrifuged at $350 \times \mathrm{g}, 4^{\circ} \mathrm{C}$ for $10 \mathrm{~min}$, and the pellet was re-suspended in $6 \mathrm{ml}$ of $30 \%$ Percoll. Mononuclear cells were separated on a three-phase Percoll gradient $\left(70 \%, 40 \%, 30 \%\right.$ Percoll) by centrifugation at $350 \times \mathrm{g}, 4^{\circ} \mathrm{C}$ for 20 min without stopping break, and collected with the help of a syringe. Cells were washed with PBS ( $350 \times \mathrm{g}, 4^{\circ} \mathrm{C}, 10 \mathrm{~min}$ ) and re-suspended in $1 \mathrm{ml}$ PBS or the corresponding buffer. 


\subsubsection{T cell purification}

\subsubsection{EasySep $^{T M}$ kit}

$\mathrm{CD}^{+} \mathrm{T}$ cells were purified by negative selection using the Easy Sep ${ }^{\mathrm{TM}}$ Mouse $\mathrm{T}$ cell Isolation Kit according to the manufacturer's instructions. In brief, the volume of the single cell suspension was adjusted to a concentration of $1 \cdot 10^{8}$ cells $/ \mathrm{ml}$ with $\mathrm{PBS} / 2 \% \mathrm{FCS}$ supplemented with $2 \%$ normal rat serum and placed in a $5 \mathrm{~mL}$ polystyrene tube. Per $\mathrm{ml}$ of cell suspension, $50 \mu \mathrm{l} \mathrm{T}$ cell enrichment cocktail were added and incubated for $15 \mathrm{~min}$ at $4^{\circ} \mathrm{C}$. Then, cells were incubated for $15 \mathrm{~min}$ with $100 \mu \mathrm{l}$ of the biotin selection cocktail at $4^{\circ} \mathrm{C}$, followed by a last incubation step of 5 min with $75 \mu \mathrm{l}$ of magnetic beads. The cell suspension was brought to a volume of $2.5 \mathrm{ml}$ with $\mathrm{PBS} / 2 \% \mathrm{FCS}$, and the tube was placed into an Easy Sep ${ }^{\mathrm{TM}}$ magnet. After $5 \mathrm{~min}$, the liquid content of the tube was decanted without removing it from the magnet. The collected suspension, corresponding to the negative fraction containing the purified T cells, was washed with PBS $\left(300 \times g, 4^{\circ} \mathrm{C}, 7 \mathrm{~min}\right)$ and the pellets were re-suspended in the corresponding buffer to determine cell numbers.

\subsubsection{Magnetic Activated Cell Sorting (MACS)}

For MACS separation, single cell suspensions were washed with MACS buffer and the Pan T cell isolation Kit II was employed according to the manufacturer's instructions. For $1 \cdot 10^{7}$ total cells, suspensions were incubated for $10 \mathrm{~min}$ at $4^{\circ} \mathrm{C}$ with $10 \mu \mathrm{l}$ of Biotin-Antibody Cocktail in $40 \mu \mathrm{l}$ MACS buffer. Without washing, $30 \mu \mathrm{l}$ of MACS buffer and $20 \mu \mathrm{l}$ of anti-biotin magnetic beads were added and incubated for 15 more min. After incubation, cells were washed in $2 \mathrm{ml}$ MACS buffer $\left(300 \times \mathrm{g}, 4^{\circ} \mathrm{C}, 7 \mathrm{~min}\right)$, re-suspended in $600 \mu \mathrm{l}$ MACS buffer and filtered to avoid cell clusters. The negative cell fraction, corresponding to T cells, was collected with the AutoMACS device, and the purified T cells were subsequently washed $\left(300 \times \mathrm{g}, 4^{\circ} \mathrm{C}, 7 \mathrm{~min}\right.$ ) and re-suspended in PBS. 


\subsubsection{Macrophage isolation and culture}

\subsubsection{Induction of Bone Marrow-Derived Macrophages (BMDMs)}

BMDMs were obtained by culturing bone marrow extracted from mouse tibiae and femur in the presence of macrophage colony-stimulating factor (M-SCF), a lineage-specific growth factor responsible for differentiation of committed myeloid progenitors into cells of the monocyte/macrophage lineage. M-SCF is secreted by 2929 cells, and therefore L929conditioned medium (LCCM) can be used to achieve macrophage maturity. In order to produce LCCM for our experiments, 2929 cells were cultured in DMEM in a $5 \% \mathrm{CO}_{2}$ atmosphere at $37^{\circ} \mathrm{C}$. Cells were split once they reached confluence, and the culture medium was collected, filtered and stored at $-20^{\circ} \mathrm{C}$.

Bone Marrow isolation. Mice were sacrificed with $\mathrm{CO}_{2}$ and the muscle tissue from the hind limbs was removed using a scalpel to obtain clean femur and tibiae. Both ends of the bone were cut and the bone marrow was flushed with ice-cold PBS/BSA using a $5 \mathrm{ml}$ syringe and collected in a Petri dish. Bone marrow cells were homogenized with the help of the syringe in order to obtain a single-cell suspension, which was filtered through a $40 \mu \mathrm{m}$ nylon strainer.

Macrophage culture and maturation. The cell suspensions were washed with PBS/BSA (300 x $\left.\mathrm{g}, 4^{\circ} \mathrm{C}, 7 \mathrm{~min}\right)$. The pellet was re-suspended in pre-warmed DMEM at a density of $1 \cdot 10^{6}$ cells $/ \mathrm{ml}$ and the bone marrow hematopoietic precursors were incubated $\mathrm{O} / \mathrm{N}$ on cell culture dishes to allow fibroblasts and stromal cells to adhere. Non-adherent cells were collected in the morning, washed and plated in LCCM at a concentration of $2 \cdot 10^{6}$ cells $/ \mathrm{ml}$ in $10 \mathrm{~cm}$ Petri dishes. The myeloid progenitors were incubated at $37^{\circ} \mathrm{C}$ and $5 \% \mathrm{CO}_{2}$ for $7-9$ days, and $4 \mathrm{ml}$ of fresh LCCM was added at day 4 to boost maturation. When macrophage differentiation was complete (mature macrophages can be recognized under the light microscope by their spindle shape), the cells were harvested by incubating them with $2 \mathrm{ml}$ PBS/BSA + 2mM EDTA for $20 \mathrm{~min}$ at $4^{\circ} \mathrm{C}$. Afterwards, macrophages were washed and re-suspended in DMEM for further culturing or FACS analysis. 


\subsubsection{Induction and isolation of peritoneal macrophages}

Another source of macrophages for FACS analysis and cell culture is the peritoneum. Peritoneal macrophages were elicited by i.p. injection of $1 \mathrm{ml}$ of thioglycolate, a monosaccharide frequently used to induce neutrophil and macrophage responses in vivo. 4 days after injection, a peritoneal lavage with PBS/BSA was performed with the help of a Pasteur pipette. The collected cell suspension was washed in PBS via centrifugation $(300 \times \mathrm{g}$, $4^{\circ} \mathrm{C}$ for $7 \mathrm{~min}$ ) and pellets were re-suspended in $8 \mathrm{ml} \mathrm{DMEM}$ and plated in $10 \mathrm{~cm}$ plates to let macrophages adhere. After $1 \mathrm{~h}$ incubation at $37^{\circ} \mathrm{C}$ in a $5 \% \mathrm{CO}_{2}$ atmosphere, the plates were washed twice with PBS in order to remove non-adherent cells and debris, and $2 \mathrm{ml}$ of PBS/EDTA were added to promote macrophage detachment. Plates were incubated for 20 min and macrophages were collected and washed with PBS ( $300 \times \mathrm{g}, 4^{\circ} \mathrm{C}$ for $\left.7 \mathrm{~min}\right)$. Pellets were re-suspended in $1 \mathrm{ml}$ PBS/BSA or the corresponding buffer, and cell numbers were determined as previously described.

\subsubsection{In vitro macrophage stimulation}

When a pre-activated state of macrophages was needed, M1 polarization was induced on naive BMDMs incubating them for $24 \mathrm{~h}$ in DMEM supplemented with $20 \mathrm{ng} / \mathrm{ml}$ LPS and 50 ng/ml IFNy. Afterwards, cells were washed once with PBS/BSA and prepared for further use.

\subsubsection{CFSE staining}

CFSE is a fluorescent dye that covalently binds to lysine residues and other amines, crosslinking to intracellular proteins. Due to its high stability, it serves to track cell proliferation by FACS, since every cell division halves the fluorescence in the daughter cells. For the T cell proliferation experiments described in this thesis, purified T cells were diluted in PBS at a concentration of $1.10^{6}$ cells $/ \mathrm{ml}$ and incubated in a water bath at $37^{\circ} \mathrm{C}$ with $0.25 \mu \mathrm{M}$ of CSFE for 10 min. Every minute, cells were gently mixed by inverting the tubes. The reaction was stopped by adding FCS till a final concentration of $2 \%$. Subsequently, cells were washed twice $\left(300 \times \mathrm{g}, 4^{\circ} \mathrm{C}\right.$ for $7 \mathrm{~min}$ ) and re-suspended in PBS or the corresponding medium. 


\subsubsection{Flow cyotometry (FACS)}

\subsubsection{Extracellular staining}

Single cell suspensions subjected to FACS analysis were prepared at an approximate concentration of $5 \cdot 10^{5}$ cells $/ \mathrm{ml}$ in FACS buffer. Cells were firstly incubated with Fc Block ${ }^{\mathrm{TM}}$ (anti-mouse CD16/CD32) for 20 min to prevent unspecific binding. Subsequently, cells were incubated with the respective fluorescence-labeled Abs for $20 \mathrm{~min}$ in the dark. After incubation, cells were washed with $3 \mathrm{ml} \mathrm{FACS}$ buffer ( $350 \times \mathrm{g}, 5 \mathrm{~min})$ and analyzed in the FACS machine.

\subsubsection{Intracellular staining}

For nuclear staining of FoxP3 the Intracellular Staining Kit from eBioscience was employed. First, $1 \cdot 10^{6}$ cells were stained with the surface Abs (CD3, CD4 and CD25) following the abovementioned protocol. Afterwards, cells were incubated for 30 min at $4{ }^{\circ} \mathrm{C}$ with $100 \mu \mathrm{l}$ of cold Fix/Perm buffer and washed with $2 \mathrm{ml}$ PBS (7 min, $350 \times \mathrm{g}$ ). Two more washing steps with 2 $\mathrm{ml}$ of freshly diluted Perm Wash buffer were done before incubation with the anti-mouse FoxP3 Abs (30 min, $4{ }^{\circ} \mathrm{C}$ in the dark). Finally, cells were washed once more with Perm Wash buffer and analyzed in the FACS machine.

\subsubsection{Annexin V apoptosis staining}

Apoptotic cells were detected by an additional incubation step after normal surface antigen staining. Cells were washed in FACS buffer ( 350 x g, $5 \mathrm{~min}$ ) and incubated for 15 min with $100 \mu$ of $1 x$ Annexin-buffer containing $1 \mu$ of fluorescent-labeled Annexin $V$, a protein which binds to phosphatidylserine residues that are exposed on the surface of early apoptotic cells. Cells were then analyzed in the FACS device without any additional washing. 


\subsection{In vitro assays}

\subsubsection{In vitro $\mathrm{T}$ cell apoptosis}

$1 \cdot 10^{5}$ splenocytes from wt C57BL/6 mice were cultured in 96 well plates in the presence of PBS, $1 \cdot 10^{-6} \mathrm{M}$ and $1 \cdot 10^{-7} \mathrm{M}$ Dex, ENPs or $1 \cdot 10^{-6} \mathrm{M}$ and $1 \cdot 10^{-7} \mathrm{M}$ BNPs. Cells were collected $5 \mathrm{~h}$, $10 \mathrm{~h}$ and $20 \mathrm{~h}$ post-treatment and the percentage of apoptotic T cells was determined via FACS analysis gating on $\mathrm{CD}^{+} / \mathrm{AnnexinV}^{+-}$population.

\subsubsection{In vitro nanoparticle distribution in mixed cultures}

The cell distribution of the BNPs was analyzed in vitro in cultures of splenocytes and lymph node cells. $1 \cdot 10^{5}$ cells were plated in 96 well plates and treated with $1 \cdot 10^{-6} \mathrm{M}$ BNPs. $6 \mathrm{~h}, 24 \mathrm{~h}$ and $48 \mathrm{~h}$ later, cells were collected and nanoparticle uptake was determined in different cell subsets $\left(\mathrm{CD}^{+}, \mathrm{B} 220^{+}\right.$and $\mathrm{CD} 11 \mathrm{~b}^{+}$cells) by FACS analysis via quantification of the FITC fluorescence emitted by each population.

\subsubsection{Ex vivo re-stimulation of MOG-specific effector T cells}

$M R^{f l}$ (control) and $M R^{l y s M}$ mice were immunized and at day 10 after immunization the mice were sacrificed to obtain single cell suspensions from spleens and lymph nodes. $6 \cdot 10^{5}$ splenocytes and $3 \cdot 10^{5}$ lymph node cells were incubated in ReMed in the presence of $20 \mu \mathrm{M}$ $\mathrm{MOG}_{35-55}$ for $72 \mathrm{~h}\left(37^{\circ} \mathrm{C}, 5 \% \mathrm{CO}_{2} ; 96\right.$-well U-bottom plates in a total volume of $\left.110 \mu \mathrm{l}\right)$. After that, the supernatants were collected, centrifuged to remove cell debris $\left(300 \times \mathrm{g}, 4^{\circ} \mathrm{C}\right.$ for 7 $\min$ ) and stored at $-20^{\circ} \mathrm{C}$ to be used for ELISA. 


\subsubsection{BMDM-mediated activation of MOG-specific T cells}

The antigen presenting capacity of $M R^{\mathrm{lySM}}$ macrophages was evaluated with the following experimental setting. $B M D M s$ from $M^{f l}$ (control) and $M R^{l y s M}$ mice at day 7 of their maturation state were plated in 96 well plates at $1 \cdot 10^{5}$ cells/well. Afterwards, $1 \cdot 10^{5} \mathrm{CSFE}$ labeled T cells, freshly isolated from 2D2 RFP mice, were added. Both cell types were cocultured in Re-Med in the presence or absence of $20 \mu \mathrm{M} \mathrm{MOG} 35-55$, and $\mathrm{T}$ cells were harvested after $24 \mathrm{~h}, 48 \mathrm{~h}$ and $72 \mathrm{~h}$ to analyze their proliferation by FACS. Cell culture supernatants were stored at $-20^{\circ} \mathrm{C}$ for cytokine quantification.

With a similar setup regarding BMDMs culture and stimulation, additional experiments using in vitro re-stimulated RFP Th17 MOG-specific cells were also performed in order to analyze the IL-17A secretion potential upon MR ${ }^{\text {lysM }}$ BMDM activation. RFP Th17 MOG-specific cells were kindly provided by Judith Strau $\beta$, from the IMSF in Göttingen.

\subsection{Histology}

\subsubsection{Isolation and fixation of spinal cords}

Mice were euthanized with $\mathrm{CO}_{2}$ and perfused with $20 \mathrm{ml}$ of saline solution to clean vessels from blood. Then, 4\% PFA was circulated throughout the body to fix the tissue. Spinal cords were isolated, kept in $4 \%$ PFA for $48 \mathrm{~h}$ and stored in PBS at $4^{\circ} \mathrm{C}$.

Whole spinal cords were cut in smaller sections, mounted in a grid in PBS and dehydrated $\mathrm{O} / \mathrm{N}$. Then the tissue was embedded in paraffin blocks and $3 \mu \mathrm{m}$ sections of the spinal cord were cut with the microtome. 


\subsubsection{Immunohistochemistry (IHC)}

IHC staining of the sections was performed as follows: first, sections were re-hydrated immersing the slides through a decreasing alcohol gradient (xylol, ethanol 99\%, ethanol 96\%, ethanol $70 \%$ and $\mathrm{ddH}_{2} \mathrm{O}$ ). For antigen retrieval, the slides were then boiled in citrate buffer for 15 min, washed with $\mathrm{ddH}_{2} \mathrm{O}$ and PBS, and endogenous peroxidase activity was blocked with $3 \% \mathrm{H}_{2} \mathrm{O}_{2}$ for $15 \mathrm{~min}$ at $4^{\circ} \mathrm{C}$. Subsequently, another blocking step with PBS/FCS was performed at $\mathrm{RT}$, and the slides were incubated $\mathrm{O} / \mathrm{N}$ at $4^{\circ} \mathrm{C}$ with the primary $\mathrm{Abs}(1: 200 \mathrm{in}$ PBS/FCS). After washing with PBS, the slides were incubated $1 \mathrm{~h}$ at RT with the secondary Abs (anti-rat-lgG-biotin, 1:200 in PBS/FCS) and afterwards with Streptavidin-peroxidase (1:1000 in PBS/FCS) for one more hour. Then, the slides were developed with DAB at RT for 1-2 $\mathrm{min}$, and the reaction was stopped with $\mathrm{ddH}_{2} \mathrm{O}$. Finally, the sections were dehydrated again in an increasing alcohol gradient and the slides were covered with Entellan.

\subsubsection{Luxol fast blue Periodic acid-Schiff (LFB-PAS)}

To determine the de-myelination grade in the spinal cord of mice with ongoing EAE, LFB-PAS staining was performed. Firstly, the spinal cord sections were immersed in decreasing alcohol concentrations and the gradient was stopped at $96 \%$ ethanol. The slides were then incubated $\mathrm{O} / \mathrm{N}$ at $56^{\circ} \mathrm{C}$ in a $0.1 \%$ LFB solution. LFB stains the myelin in blue. After washing the slides with $96 \%$ ethanol and $\mathrm{ddH}_{2} \mathrm{O}$, excess of $\mathrm{LFB}$ was removed immersing them in $0.1 \%$ $\mathrm{Li}_{2} \mathrm{CO}_{3}$ for $30 \mathrm{~s}$, followed by washing in $70 \%$ ethanol and $\mathrm{ddH}_{2} \mathrm{O}$. Then, slides were incubated during 10 min in $0.8 \%$ Periodic Acid, washed in $\mathrm{ddH}_{2} \mathrm{O}$, and 20 more min in Schiff's reagent, the excess of which was washed away with a sulphite wash. Finally, the cuts were washed 10 min in tap water, dehydrated and mounted as previously described. 


\subsubsection{Bielschowsky silver staining}

Silver impregnation was performed to stain axonal fibers using a modified protocol based on Litchfield and colleagues (Litchfield and Nagy 2001). Slides were first de-parafined in a decreasing alcohol gradient and immersed for $15 \mathrm{~min}$ in a $20 \% \mathrm{AgNO}_{3}$ solution at $4{ }^{\circ} \mathrm{C}$. Then, slides were collected in $\mathrm{ddH}_{2} \mathrm{O}$ and $25 \% \mathrm{NH}_{3}$ solution was added drop by drop to the $\mathrm{AgNO}_{3}$ until the brown precipitate disappeared. Slides were then incubated for $20 \mathrm{~min}$ at $4^{\circ} \mathrm{C}$ in the newly formed silver hydroxide solution, and washed in ammonium water for $5 \mathrm{~min}$. The staining was then developed immersing the slides in the previously used ammoniacal silver solution with the developer ( $50 \mu \mathrm{l}$ developer / $25 \mathrm{ml}$ Ag solution). When the nerve fibers got dark, slides were washed with ammonium water and $\mathrm{dd}_{2} \mathrm{O}$. Finally, cuts were incubated 5 min in sodium thiosulfate at $4^{\circ} \mathrm{C}$, dehydrated in an increasing alcohol gradient, and covered with Entellan.

All reagents needed for the various histological staining protocols were kindly provided by the IMSF in Göttingen.

\subsection{Molecular methods}

\subsubsection{DNA isolation from biopsies}

Tail samples were digested $\mathrm{O} / \mathrm{N}$ by incubation at $56^{\circ} \mathrm{C}$ in $750 \mu \mathrm{L}$ of Tail buffer with $20 \mu \mathrm{L}$ proteinase $\mathrm{K}$. After vortexing, $300 \mu \mathrm{L}$ from a saturated $\mathrm{NaCl}$ solution were added, incubated for $5 \mathrm{~min}$ at RT and the samples were centrifuged for $10 \mathrm{~min}$ at $20.800 \times \mathrm{g}$. The upper phase of each sample was removed and mixed with $600 \mu \mathrm{L}$ isopropanol, incubated for $3 \mathrm{~min}$ at RT and again centrifuged for $10 \mathrm{~min}$ at $20.800 \mathrm{xg}$. The supernatant was discarded and $500 \mu \mathrm{L}$ of $70 \% \mathrm{EtOH}$ were added, followed by further centrifugation for $5 \mathrm{~min}$. The pellet was dried and dissolved in $100 \mu \mathrm{L}$ TE buffer. The samples were stored at $4{ }^{\circ} \mathrm{C}$. 


\subsubsection{Polymerase chain reaction (PCR)}

DNA samples from newborn transgenic mice were subjected to PCR amplification with specific primers in order to determine their genotypes.

Table 1.11 |PCR reagents and thermocycler program.

PCR reaction mix PCR program

\begin{tabular}{|c|c|c|c|c|}
\hline $0.5 \mu \mathrm{L}$ DNA & $1 \mathrm{~min}$ & $98.5^{\circ} \mathrm{C}$ & Activation & \multirow{4}{*}{$\times 30$} \\
\hline $4 \mu \mathrm{L}$ High Fidelity buffer & $20 s$ & $98.5^{\circ} \mathrm{C}$ & Denaturation & \\
\hline $1 \mu \mathrm{L}$ dNTPs $5 \mathrm{mM}$ & $15 \mathrm{~s}$ & $64{ }^{\circ} \mathrm{C}$ & Annealing & \\
\hline $1 \mu \mathrm{L}$ Primer mix & $20 \mathrm{~s}$ & $72{ }^{\circ} \mathrm{C}$ & Elongation & \\
\hline $0.3 \mu \mathrm{L}$ PhuS & $2 \mathrm{~min}$ & $72{ }^{\circ} \mathrm{C}$ & & \\
\hline $13.2 \mu \mathrm{L} \mathrm{dH_{2 }} \mathrm{O}$ & & & & \\
\hline
\end{tabular}

Amplification products were run together with $7 \mu \mathrm{L}$ of the DNA loading dye Orange $\mathrm{G}$ on a $1.5 \%$ agarose gel at $120 \mathrm{~V}, 230 \mathrm{~mA}$ for $25 \mathrm{~min}$.

\subsubsection{RNA isolation}

RNA extraction from frozen tissue was performed with the help of the RNeasy plus Universal Kit (Quiagen). The tissue samples were homogenized in $900 \mu \mathrm{L}$ Quiazol using an Ultra-Turrax mixer, then $100 \mu \mathrm{L}$ DNA Eliminator solution and $180 \mu \mathrm{L}$ chloroform were added before centrifugation at $20.800 \times \mathrm{g}, 4^{\circ} \mathrm{C}$ for $15 \mathrm{~min}$. The upper phase was mixed with $600 \mu \mathrm{L} 70 \%$ EtOH by pipetting and poured into a RNA-separation column which was centrifuged for $15 \mathrm{~s}$. Subsequently, $700 \mu \mathrm{L}$ RWT buffer were added to the column, which was centrifuged for $20 \mathrm{~s}$ at $20.800 \times \mathrm{g}$. The process was repeated with $500 \mu \mathrm{L}$ RPE buffer for $2 \mathrm{~min}$, plus 1 additional

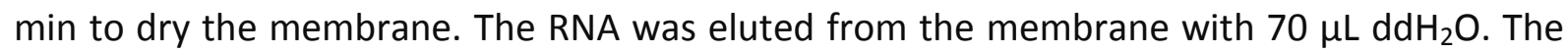
RNA concentration was quantified with the Nanodrop. Additionally, $1 \mu \mathrm{g}$ RNA was run on a $1 \%$ agarose gel to check its purity on the basis of the $18 \mathrm{~s}$ and $28 \mathrm{~s}$ rRNA bands. 
In the case of RNA purification from cell cultures, cells were directly lysed on the wells with a lysing solution and the Quick-RNA MiniPrep kit (Zymo Research), with comparable steps as the above described, was used according to the manufacturer's instructions.

\subsection{4 cDNA synthesis}

In order to quantify gene expression $1 \mu \mathrm{g}$ RNA was reversely transcribed with the iScript Reaction Mix kit. $4 \mu \mathrm{l}$ of iScript buffer and $0.25 \mu$ l Reverse Transcriptase were added per reaction tube, and volume was adjusted to $20 \mu l$. The cDNA synthesis was performed in a thermocycler with a three-step incubation program: $5 \mathrm{~min}$ at $25^{\circ} \mathrm{C}, 30 \mathrm{~min}$ at $42^{\circ} \mathrm{C}$ and $5 \mathrm{~min}$ at $85^{\circ} \mathrm{C}$.

\subsubsection{Quantitative real-time PCR (qRT-PCR)}

qRT-PCR was performed to determine the gene expression levels of certain genes (primers in Table 1.9) with the Power SYBR Green polymerase according to manufacturer's instructions.

Table 1.12 |qRT-PCR reagents and thermocycler program

qRT-PCR reaction mix

$1 \mu \mathrm{L}$ CDNA

$12.5 \mu \mathrm{L}$ SYBR Green

$11 \mu \mathrm{LddH} \mathrm{H}_{2} \mathrm{O}$

$0.5 \mu \mathrm{L}$ Primer mix
$2 \min$

$10 \min$

$15 \mathrm{~s}$

$1 \mathrm{~min}$

$15 \mathrm{~s}$

$1 \mathrm{~min}$

$15 \mathrm{~s}$

qRT-PCR program

$50{ }^{\circ} \mathrm{C}$

$95{ }^{\circ} \mathrm{C}$

$95{ }^{\circ} \mathrm{C}$

$60{ }^{\circ} \mathrm{C}$

$95{ }^{\circ} \mathrm{C}$

$60{ }^{\circ} \mathrm{C}$

$95{ }^{\circ} \mathrm{C}$
Activation

Denaturation

Denaturation

Annealing + Elongation

$x 40$ $\times 40$

.




\subsubsection{Enzyme-linked immuno-absorbent assay (ELISA)}

ELISA was performed on appropriately diluted supernatants from re-stimulated $\mathrm{T}$ cell, macrophage or mixed cell cultures according to the manufacturer's instructions. In brief, 96well optic plates were coated $\mathrm{O} / \mathrm{N}$ with $100 \mu$ l capture antibody (1:200 dilution in coatingbuffer). Between incubation steps, wells were washed 4 times with $200 \mu \mathrm{l} \mathrm{PBS/Tween.}$

The following morning, plates were blocked with Assay Diluent for $1 \mathrm{~h}$. Then, serial dilutions of the standard provided by the kit and the corresponding samples were loaded on the wells and incubated for $2 \mathrm{~h}(100 \mu \mathrm{l} /$ well, all samples were run in triplicates). Afterwards, wells were incubated $1 \mathrm{~h}$ with the detection Abs (1:200 in Assay Diluent) and after subsequent washing steps, additionally 30 min with Horse Radish Peroxidase (HRP, 1:1000 in Assay Diluent). Finally, $100 \mu \mathrm{l} /$ well of substrate solution were added and a blue color with an intensity proportional to the protein concentration developed along $20 \mathrm{~min}$ in the dark. To stop the reaction, $100 \mu \mathrm{l} /$ well of $2 \mathrm{~N} \mathrm{H}_{2} \mathrm{SO}_{4}$ were added and the plate was measured in the photometer. Final concentration of the protein in the samples was calculated via extrapolation of the absorbance values from the standard dilutions.

\subsubsection{Cytometric Bead Array (CBA)}

Th1, Th2 and Th17 cytokines were quantified in undiluted cell culture supernatants with a CBA. This method allows the simultaneous detection of different cytokines thanks to bead populations with different PerCP-intensities coated with specific capture antibodies for each cytokine. All reagents used were provided by the kit. First, the capture beads for each cytokine were mixed in equal proportions, and standards were prepared performing 1:2 serial dilutions from the top standard. Then, a 96 well plate was pre-wet with $100 \mu \mathrm{l} /$ well of wash buffer, and $20 \mu \mathrm{l} /$ well of the mixed capture beads, together with $20 \mu \mathrm{l}$ of the samples and $20 \mu \mathrm{l}$ of the PE detection reagent were incubated for $2 \mathrm{~h}$ at RT in the dark. Then the plate was washed, samples were re-suspended in wash buffer and acquired in the FACS device. Data analysis was performed using the FCAP Array software v.3.0.1 (BD Bioscience). 


\subsection{Statistical analysis}

Data was analyzed by two-tailed Student's t test for unpaired groups using GraphPad Prism 5.03 (San Diego, California, USA). The Mann Whitney test with the Wilcoxon correction was used in the case of the EAE scoring curves. Results are depicted as mean values $\pm S E M ; p$ values above 0.05 were considered as non-significant (n.s.); ${ }^{*}, \mathrm{p}<0.05 ; * *, p<0.01$; and ${ }^{* * *}$, $\mathrm{p}<0.001$. 


\section{RESULTS}

\subsection{EAE therapy with betamethasone nanoparticles}

In recent years, the use of new carriers for the selective delivery of drugs to treat cancer and inflammatory diseases, such as MS, has attracted widespread attention. Previous research from our group revealed that liposomal encapsulation altered the mechanism of action of GCs, now targeting macrophages to a larger extent than T cells (Schweingruber et al. 2011). In addition, the potency of the drug in the treatment of EAE was increased. It is against this background that we investigated IOH-NPs (Heck et al. 2015) as an alternative vehicle for the delivery of GCs specifically to myeloid cells. Hence, the first part of this work was aimed to explore the features of BNPs in different cellular contexts, their therapeutic efficacy and mechanism of action in the treatment of EAE, and potential side effects.

\subsubsection{BNPs are preferentially taken up by myeloid cells}

Initial work from our group on this topic showed that the efficacy of the BNPs in macrophages was similar to the one of the free GC Dex in terms of expression of proinflammatory cytokines and molecules involved in antigen presentation (Ring, unpublished data). These experiments confirmed that BNPs were well tolerated in vitro and taken up by myeloid cells. Unexpectedly, however, they also induced T cell apoptosis to the same extent as free Dex, indicating that their effect in vitro was not limited to phagocytic cells. Thus I started to compare the uptake of BNPs by different cell types in vitro using 2-photon microscopy (2-PM) and FACS, taking advantage of the fact that the fluorescent dye FMN has been incorporated into the nanoparticles. 
BMDMs, peritoneal lavage cells, splenocytes and lymph node cells were treated with BNPs for up to $48 \mathrm{~h}$ in vitro, and the uptake of the BNPs was monitored in different cell types. Analysis by 2-PM revealed the intracellular presence of the fluorescent nanoparticles in BMDMs, distributed in a homogeneous fashion (Figure 3.1 A). FACS analysis allowed to further distinguish the uptake efficacy between individual cell types. Although T cells, B cells and macrophages increasingly accumulated the fluorescent dye over time, CD11 $\mathrm{b}^{+}$myeloid cells were the brightest, indicating that they took up the BNPs more efficiently than T and B cells (Figure 3.1 B,C,D). These data collectively indicate that the nanoparticles in vitro are able to target various types of immune cells albeit with different efficacy.

A

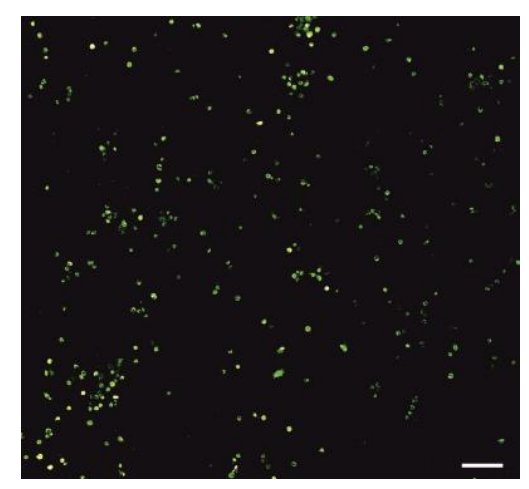

C

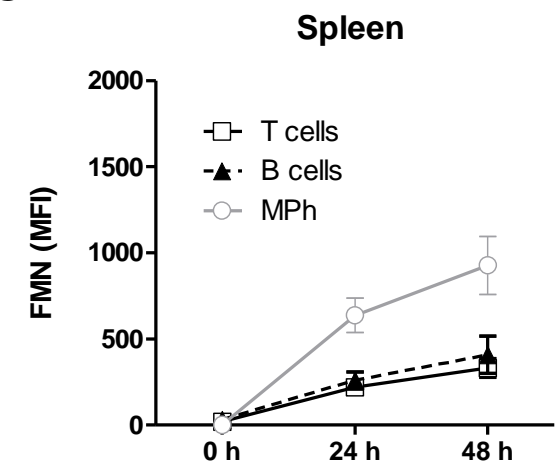

B

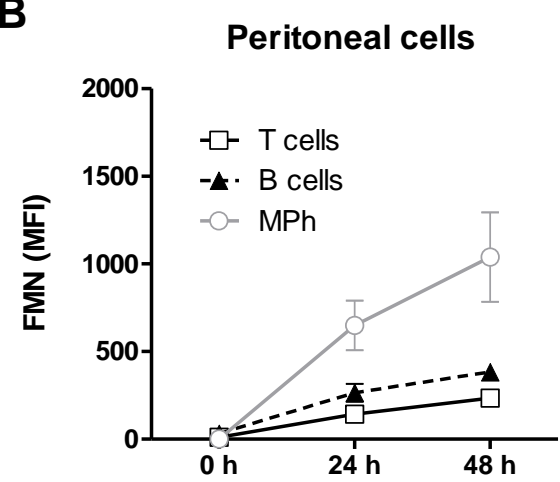

D

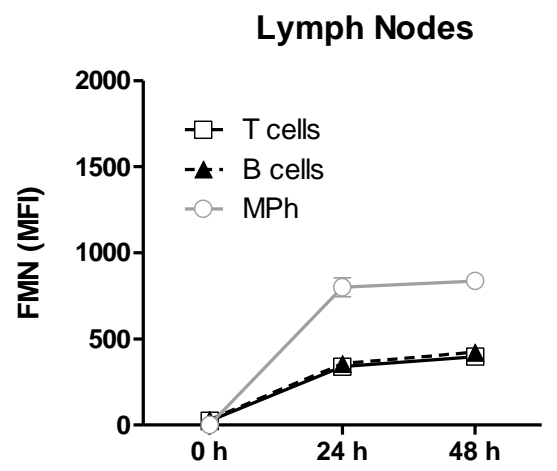

Figure 3.1 | BNPs are efficiently but differentially taken up by immune cells in vitro. (A) Analysis of BMDMs treated O/N with BNPs by 2-PM (in collaboration with Judith StrauB, IMSF Göttingen). (B,C,D) Peritoneal lavage cells, splenocytes and lymph nodes cells were isolated from wt C57BL/6 mice ( $n=6)$ and total cell suspensions were cultured in the presence of $10^{-6} \mathrm{M}$ BNPs. Cells were harvested after $24 \mathrm{~h}$ and $48 \mathrm{~h}$, 
incubated with monoclonal Abs against CD3, B220 and CD11b and analyzed by FACS. After electronic gating on the three subpopulations, nanoparticle uptake was quantified on the basis of the mean fluorescence intensity $(\mathrm{MFI})$ of the FMN dye. Values are depicted as mean \pm SEM.

\subsubsection{BNPs are more potent in modulating macrophages than $\mathrm{T}$ cell activity in vivo}

Previous in vitro analyses suggested that BNPs equally modulated T cell and macrophage function in a similar manner. However, in a competitive situation such as mixed cultures, the nanoparticles were preferentially internalized by myeloid cells. Thus we hypothesized that BNPs might exert a stronger anti-inflammatory effect on macrophages as compared to $T$ cells when applied in vivo.

First, surface expression of molecules involved in antigen-presentation was studied on peritoneal macrophages by FACS (Figure 3.2 A). In all experiments, the same dose of free Dex and BNPs was employed $(10 \mathrm{mg} / \mathrm{kg}$ ) and administered on three consecutive days. PBS or empty nanoparticles without the drug (ENPS) served as controls. Expression of MHC class II and CD86 were both strongly reduced by Dex and BNPs compared to their respective controls. To confirm the efficacy of the BNPs, peritoneal macrophages were isolated from mice treated with Dex, BNPs, PBS or ENPs for three days, and subsequently stimulated in vitro with IFNy and LPS. Secretion of TNFa was then analyzed in the supernatants by ELISA (Figure 3.2 B). Similar to the results of the FACS analysis, in vivo applied Dex and BNPS resulted in a suppression of TNF $\alpha$ release compared to the controls. Taken together, BNPs and free Dex have a comparable immunomodulatory efficacy in macrophages in vivo. 
A

MHC- II

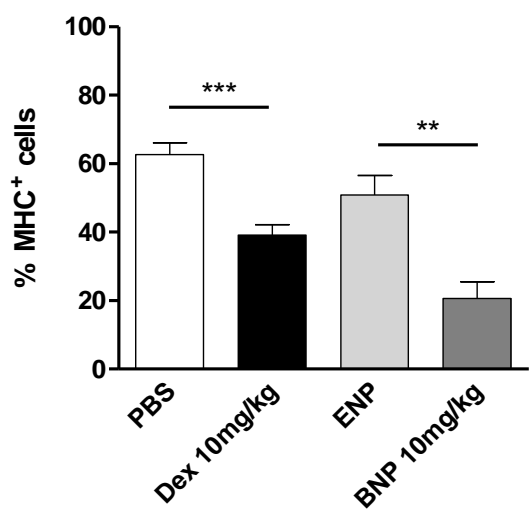

CD86

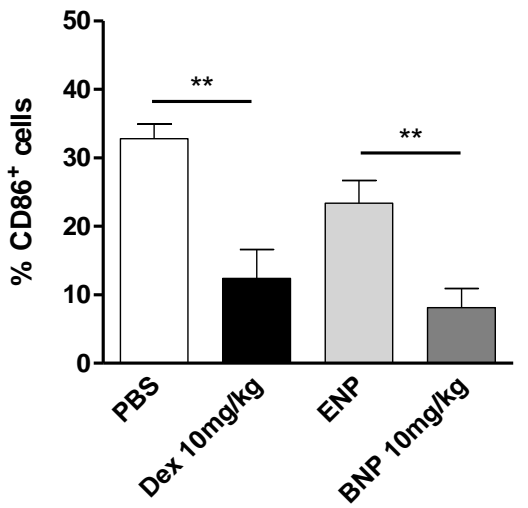

B

TNF $\alpha$

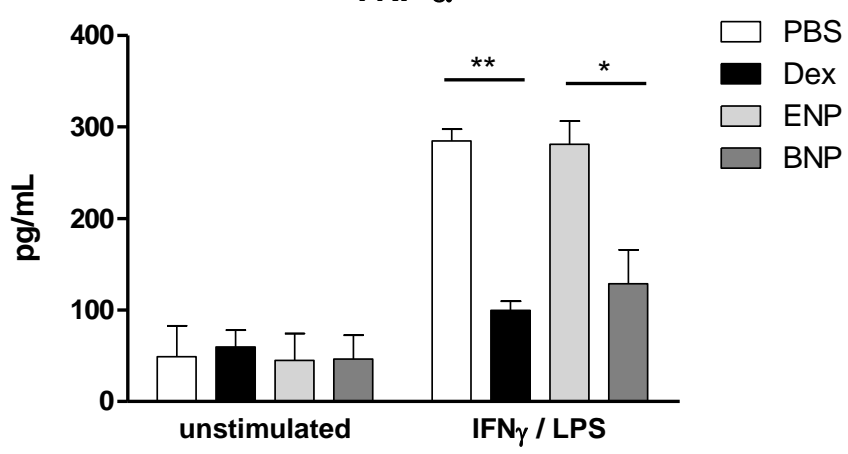

Figure 3.2 | BNPs exert potent immunosuppressive activity on peritoneal macrophages in vivo. Thioglycolate-elicited macrophages were obtained by peritoneal lavage from wt C57BL/6 mice that received a daily injection of PBS $(n=5), 10 \mathrm{mg} / \mathrm{kg}$ Dex $(n=6)$, ENPs $(n=8)$ or $10 \mathrm{mg} / \mathrm{kg}$ BNPs $(n=6)$ for three consecutive days. (A) Cells were incubated with monoclonal Abs against CD11b, IA ${ }^{\mathrm{b}}$ (MHC II) and CD86 and analyzed by FACS. Cells expressing MHC II and CD86 were determined after gating on CD11 ${ }^{+}$cells. (B) $2 \cdot 10^{5}$ peritoneal macrophages $/ \mathrm{ml}$ ( $\mathrm{n}=3$ per group) obtained from mice treated as in panel A were cultured in DMEM medium and half of the wells were stimulated with LPS and IFNY. After $48 \mathrm{~h}$, cell culture supernatants were collected and the concentration of TNF $\alpha$ was determined by ELISA. Statistical significance was calculated using the unpaired Student's t-test and values are depicted as mean \pm SEM; * $p<0.05 ; * *, p<0.01$; and $* * *, p<0.001$.

Having demonstrated the in vivo anti-inflammatory activity of the BNPs on macrophages, we wondered whether BNPs had a comparable effect regarding T cell apoptosis. Following the same protocol as for the analysis of macrophages, mice were injected daily up to three times 
i.p. with the vehicles (PBS/ENPs) or $10 \mathrm{mg} / \mathrm{kg}$ GCs (Dex/BNPs). On day 0, 1 and 3 after treatment, total splenocytes were counted, stained for T cell markers and analyzed by FACS (Figure 3.3). In vitro it was shown that BNPs and Dex exerted a similar effect on $\mathrm{T}$ cell apoptosis as free GCs. In vivo, Dex induced a reduction of total splenocytes as well as CD4 ${ }^{+}$ and $\mathrm{CD}^{+} \mathrm{T}$ cell numbers by almost $100 \%$ the third day of treatment. However, BNPs achieved only a $20 \%$ decrease in splenocyte numbers and a reduction around $30 \%$ in $\mathrm{CD} 4^{+} \mathrm{T}$ cell numbers. The amount of $\mathrm{CD} 8^{+}$cells was even unaffected. Unexpectedly, ENPs appeared to moderately increase total splenocyte numbers, in particular the $\mathrm{CD} 8^{+} \mathrm{T}$ cell subset (Figure 3.3 A). The reason and implications of this observation, however, remain unclear.

A
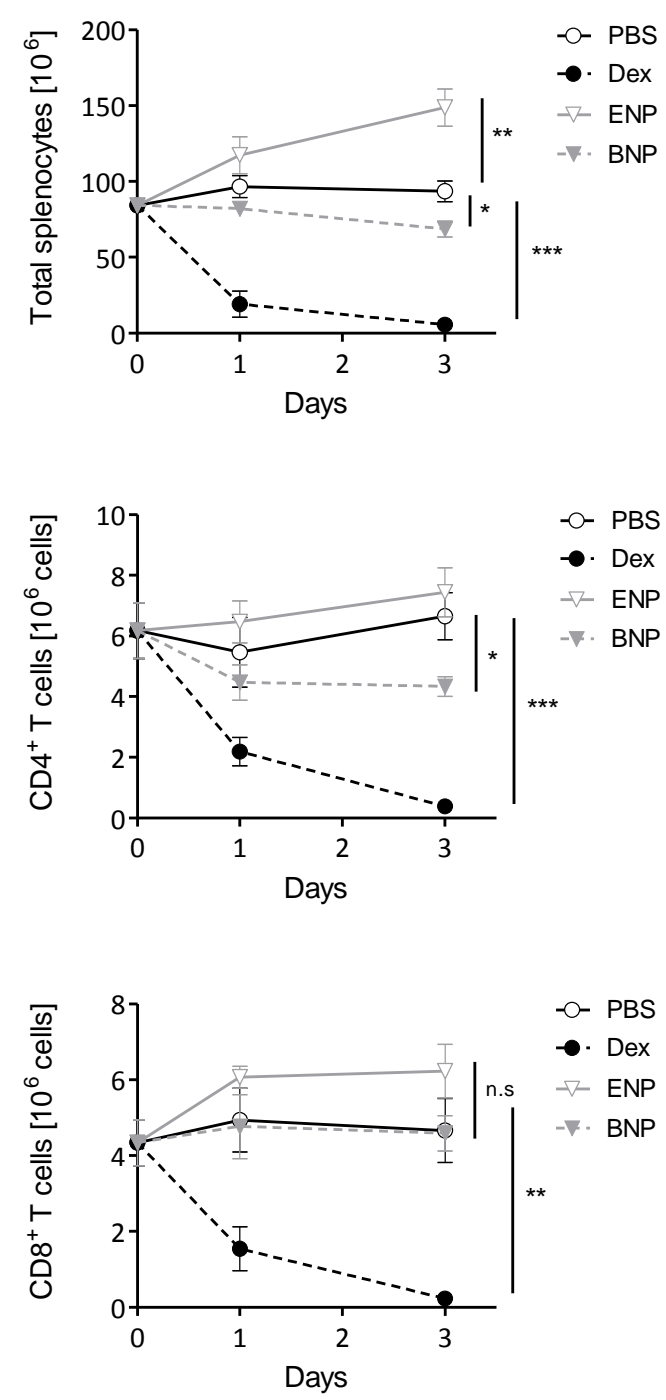

B
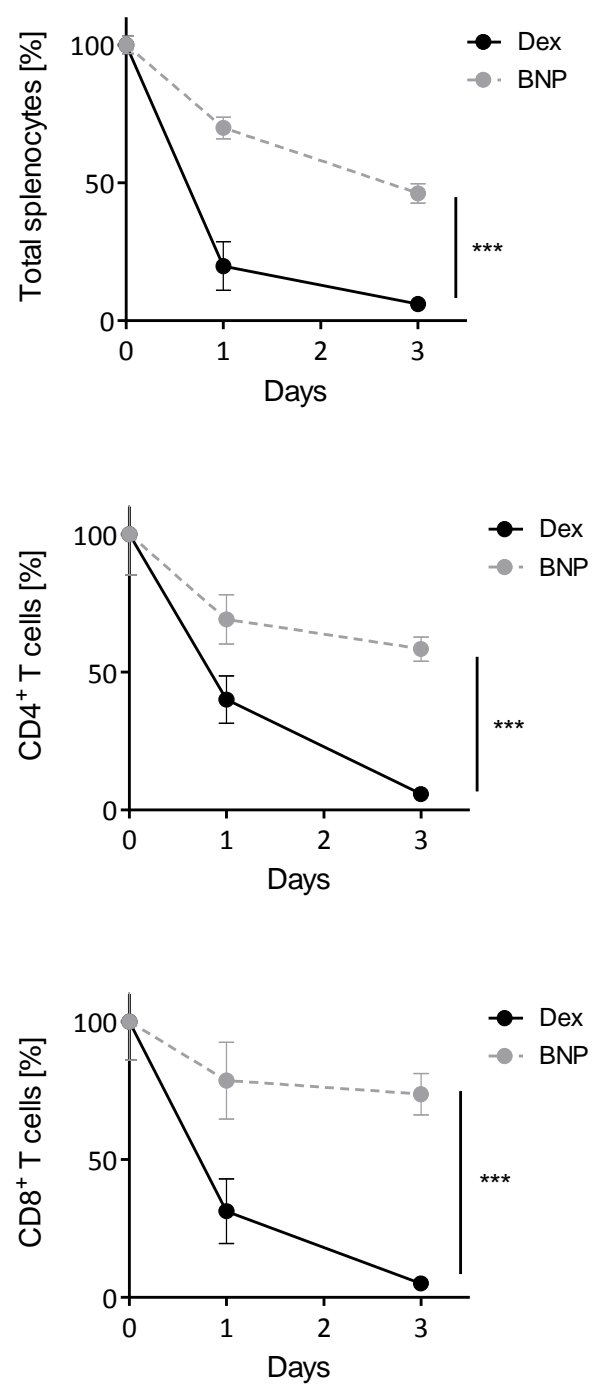
Figure 3.3 |BNPs do not efficiently induce T cell apoptosis in vivo. PBS, $10 \mathrm{mg} / \mathrm{kg}$ Dex, ENPs or $10 \mathrm{mg} / \mathrm{kg}$ BNPs were injected i.p. once per day into wt C57BL/6 mice during a three day period. On day $0(n=5), 1(n=3)$ and $3(n=6)$ after treatment, total splenocytes were isolated, counted and stained with monoclonal Abs against $\mathrm{CD} 3, \mathrm{CD} 4$ and $\mathrm{CD} 8 . \mathrm{CD}^{+}$and $\mathrm{CD} 8^{+}$populations were gated on total $\mathrm{CD}^{+} \mathrm{T}$ cells. Total cell numbers were multiplied with the percentages of each cell population, and are presented either as absolute cell numbers (A) or referred to the corresponding vehicle controls (B). Statistical significance was calculated using the unpaired Student's t-test and values are depicted as mean \pm SEM; (n.s.), p $\geq 0.05 ; *, p<0.05 ; * *, p<0.01 ;$ and $* * *, p$ $<0.001$.

\subsubsection{BNPs modulate macrophage and $T$ cell functions via the GR}

Although the findings up to this point indicated that BNPs were in principle able to modulate macrophage and $T$ cell function, the mechanism was still unknown. It is generally accepted that free GCs exert their anti-inflammatory effects mostly through binding to the GR, but it is known that selective GC activities might also be mediated by some other receptors (Funder 1997). To corroborate that the activity of BNPs was GR-dependent, the GC-nanoparticles were tested in mice with specific deletions of the GR either in macrophages or T cells.

First, GR-deficient peritoneal macrophages were analyzed for MHC class II expression after in vivo treatment using a similar protocol as in Figure 3.2. Application of Dex or BNPs to $G R^{\text {lysM }}$ mice had no effect on the surface levels of MHC class II, whereas the same treatments induced a reduction by $50 \%$ of this surface molecule in $\mathrm{GR}^{\mathrm{fl}}$ control animals, similarly to the previously shown experiments performed in mice with a wt phenotype (Figure 3.4). This indicates that the presence of the GR in macrophages is required for the function of BNPs. 
A

$\mathrm{GR}^{\mathrm{t1}}$

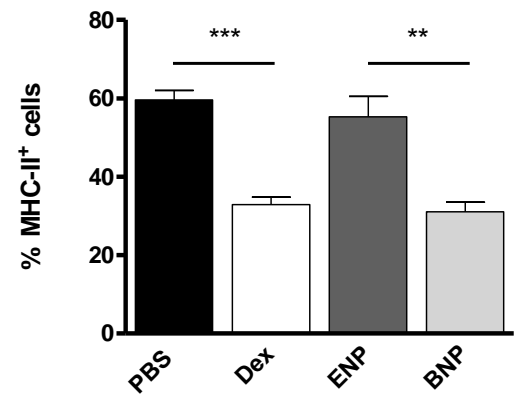

B

GR $^{\text {LysM }}$

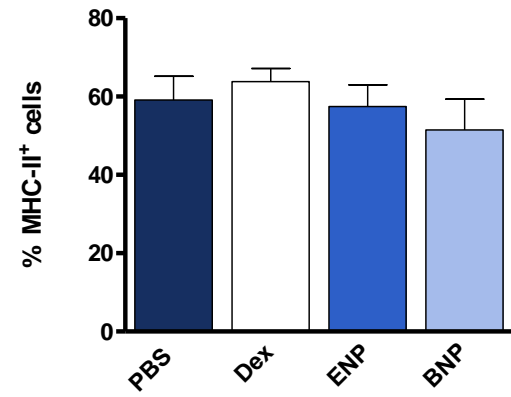

Figure 3.4 | BNPs do not reduce MHC class II expression on GRlysM macrophages in vivo. Thioglycolateelicited macrophages were obtained by peritoneal lavage from $G R^{f l}(A)$ and $G R^{l y s M}(B)$ mice that had received a daily injection of PBS ( $n=3 / 3), 10 \mathrm{mg} / \mathrm{kg}$ Dex $(n=3 / 3)$, ENPs $(n=3 / 4)$ or $10 \mathrm{mg} / \mathrm{kg}$ BNPs $(\mathrm{n}=6 / 4)$ during three consecutive days. The cells were incubated with monoclonal Abs against CD11b and IA (MHC class II). Cells expressing $\mathrm{MHC}$ class II were gated within the $\mathrm{CD}_{11} \mathrm{~b}^{+}$subpopulation. Statistical significance was calculated using the unpaired Student's t-test and values are depicted as mean $\pm \mathrm{SEM}$; ${ }^{* *}, \mathrm{p}<0.01$; and ${ }^{* * *}, \mathrm{p}<0.001$.

Furthermore, induction of $\mathrm{T}$ cell apoptosis was analyzed in vitro and in vivo. $\mathrm{GR}^{\mathrm{fl}}$ control splenocytes treated in vitro with $10^{-7} \mathrm{M}$ BNPs underwent apoptosis to the same levels as Dextreated ones, whereas the pro-apoptotic effect was abolished on splenocytes from the $G R^{\text {lck }}$ mice, with GR-deficient T cells, when they were treated with either Dex or BNPs (Figure 3.5 A,B). A similar effect was observed when Dex and BNPs were applied in vivo using a similar protocol as in Figure 1.3. The reduction in splenic T cell numbers by Dex and BNPs was more pronounced in $G R^{\text {lck }}$ mice compared to $G R^{f l}$ mice (Figure 3.5 C,D), indicating that their proapoptotic effect is partially abolished. The slight drop in T cells in the mutant mice after treatment might be due to the effect of GCs in other cell types in the spleen, such as B cells, thus causing a collateral decrease in $\mathrm{T}$ cell numbers.

Taken together, our data confirmed that not only free GCs but also BNPs require the GR in order to exert their immunomodulatory effects in T cells and macrophages. 
A

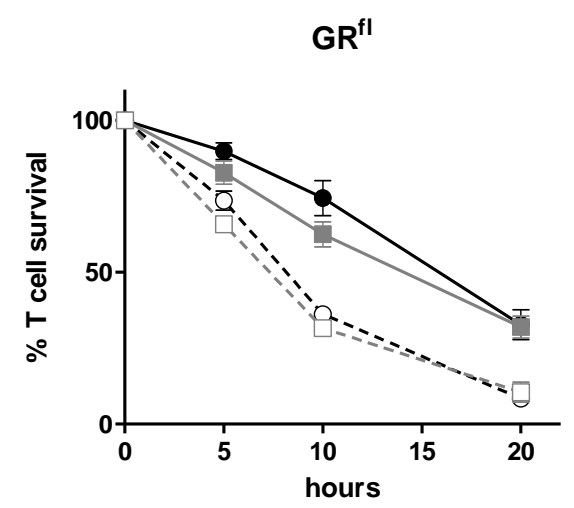

C

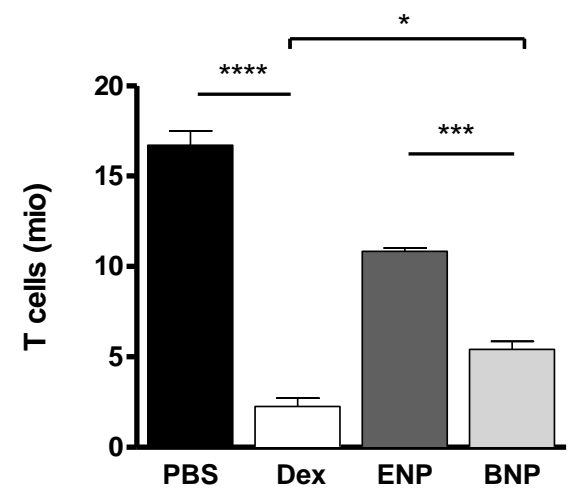

B

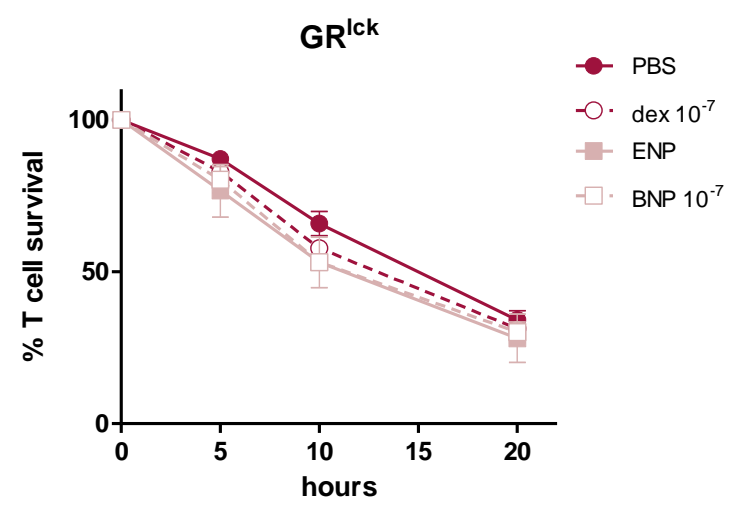

D

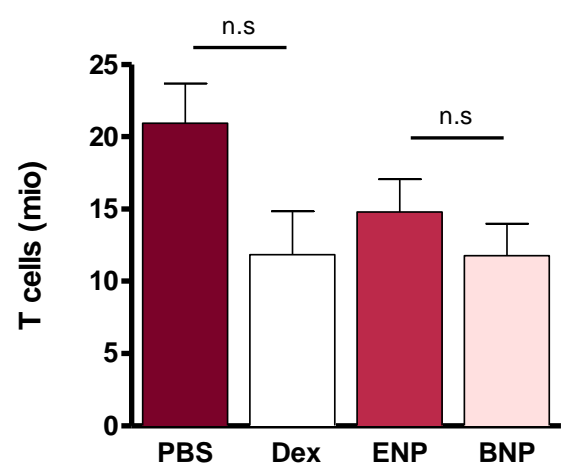

Figure 3.5 | BNPs depend on the presence of the GR for the induction of $T$ cell apoptosis. The activity of BNPs in GR-deficient T cells was evaluated in vitro and in vivo. (A, B) Total splenocytes isolated from GR ${ }^{\mathrm{fl}}$ $(n=6, A)$ and $G^{\text {lck }}(n=6, B)$ mice were cultured in vitro with PBS, $10^{-7} \mathrm{M}$ Dex, ENPs or $10^{-7} \mathrm{M} B N P$, and harvested $5 \mathrm{~h}, 10 \mathrm{~h}$ and $20 \mathrm{~h}$ after treatment. Cells were stained with an anti-CD3 Ab and Annexin V, and analyzed by FACS. The percentage of surviving T cells was referred to the values obtained for freshly isolated splenocytes, which were set to $100 \%$. (C, D) Splenocytes were isolated from $\mathrm{GR}^{\mathrm{fl}}(\mathrm{n}=6, C)$ and $G R^{\mathrm{lck}}(n=6, D)$ mice that had been treated with PBS, $10 \mathrm{mg} / \mathrm{kg}$ Dex, ENPs and $10 \mathrm{mg} / \mathrm{kg}$ BNPs once per day during a three day period. To determine total T cell numbers, the splenocytes were counted and the T cells were identified by FACS analysis of the $\mathrm{CD}^{+}$subpopulation. Statistical significance was calculated using the unpaired Student's t-test and values are depicted as mean \pm SEM; (n.s.), $p \geq 0.05 ; *^{*}, p<0.05$; and ${ }^{* * *}, p<0.001 ; * * *, p<0.0001$. 


\subsubsection{BNPs efficiently ameliorate EAE in mice}

Having proven the anti-inflammatory properties of BNPs both in vitro and in vivo, we investigated their therapeutic potential in the context of EAE. The disease was induced in $\mathrm{C} 57 \mathrm{BL} / 6$ wt mice by immunization with $50 \mu \mathrm{g}$ of $\mathrm{MOG}_{35-55}$. This form of EAE is characterized by a chronic progressive disease course that peaks at around day 14-16 post-immunization with hind limb paralysis (score 6-7), followed by partial resolution of the symptoms. C57BL/6 mice received daily doses of PBS, $10 \mathrm{mg} / \mathrm{kg}$ Dex, ENPs or $10 \mathrm{mg} / \mathrm{kg}$ BNPs on three consecutive days, starting on the day when the first clinical signs appeared (score 2-3). In order to exclude a potential difference in therapeutic efficacy between Dex and BMZ, which is contained in the nanoparticles, an additional group was treated with free BMZ. The disease curves representing the progression of clinical symptoms of EAE showed that all three GC treatment protocols (Dex, BNPs and BMZ) were equally potent in ameliorating EAE (Figure 3.6 A). Noteworthy, ENPs did not influence EAE, confirming that the vehicle had no effect in the observed phenotypes.

In order to determine the range of clinical efficacy, the amount of BNPs was titrated in the treatment of EAE. While $5 \mathrm{mg} / \mathrm{kg}$ BNPs were still able to ameliorate clinical symptoms, this was no longer the case for $2 \mathrm{mg} / \mathrm{kg}$. (Figure $3.6 \mathrm{~B}$ ). Thus, these data show the potent therapeutic effect of BNPs at $10 \mathrm{mg} / \mathrm{kg}$, and confirm a comparable efficacy to free GCs when they are applied at similar doses.

A

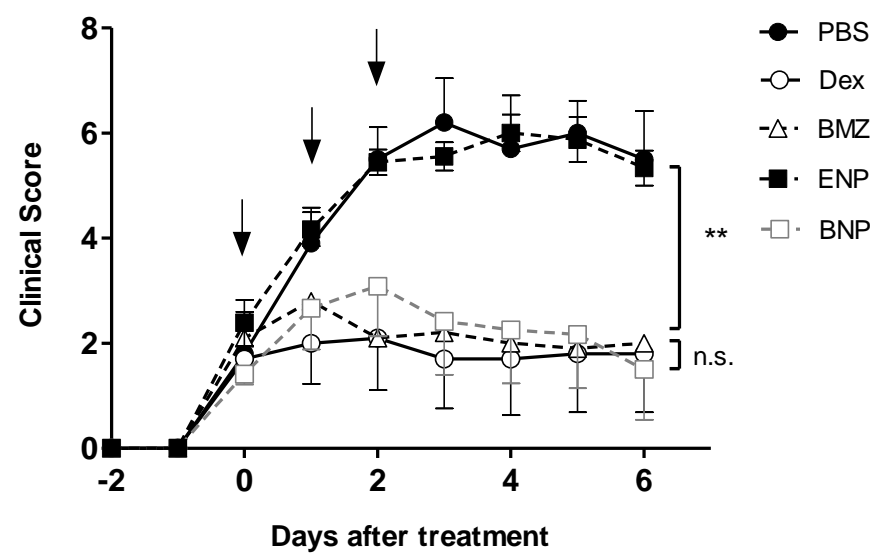




\section{B}

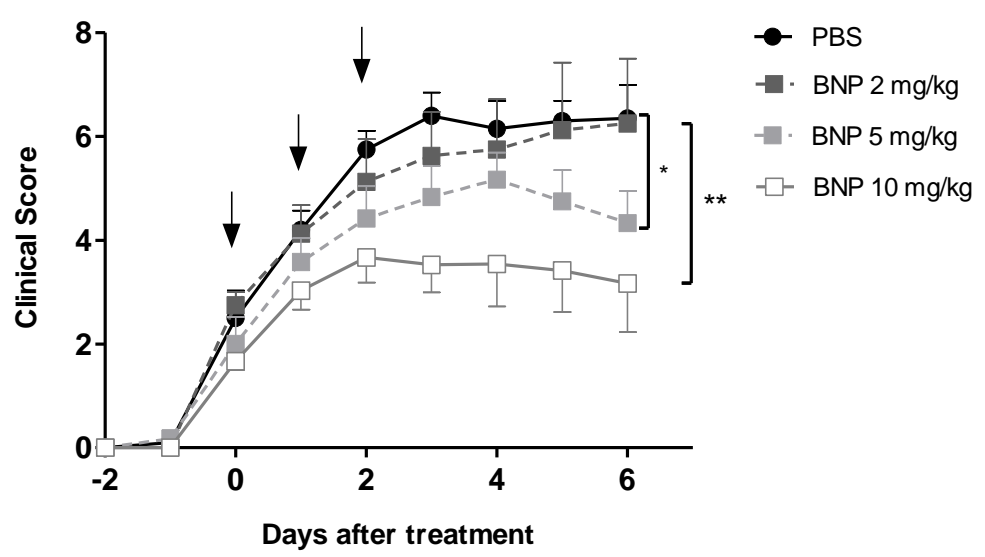

Figure 3.6 The therapeutic efficacy of BNPs in the treatment of EAE is similar to free GCs. EAE was induced by active immunization on C57BL/6 wt mice with $50 \mu \mathrm{g} \mathrm{MOG} \mathrm{MS}_{35}$ in CFA according to our standard protocol (see 2.3.1). (A) Having reached a disability score of 2-3, the mice received three consecutive daily i.p. injections of PBS ( $n=5), 10 \mathrm{mg} / \mathrm{kg}$ Dex $(n=5), 10 \mathrm{mg} / \mathrm{kg} \mathrm{BMZ} \mathrm{(n=5),} \mathrm{ENPs}(n=9)$ or $10 \mathrm{mg} / \mathrm{kg}$ BNPs (n=6) (black arrows). The severity of the disease was scored daily and is depicted on a scale from 0 to 10 . Day 0 represents the day of the first treatment in each individual mouse. (B) To titrate the amount of BNPs, different doses were administered i.p.: $2 \mathrm{mg} / \mathrm{kg}(\mathrm{n}=4), 5 \mathrm{mg} / \mathrm{kg}(\mathrm{n}=6)$ and $10 \mathrm{mg} / \mathrm{kg}(\mathrm{n}=18)$. Treatment with PBS served as a control $(n=10)$. Statistical analysis of the scoring curve was performed using the Mann-Whitney test. Values are depicted as mean \pm SEM; (n.s.), $p \geq 0.05 ;{ }^{*}, p<0.05 ; * *, p<0.01$.

The EAE model not only mimics the clinical symptoms of ascending paralysis, but also the demyelinating pathology typical for MS at the spinal cord level. This feature can be used as an independent readout of the efficacy of GC therapy. Hence, the extent of demyelination was determined in the spinal cord of mice treated with BNPs or ENPs (as control) three days after starting the therapy (Figure 3.7). Although no statistically significant difference was observed, there was a tendency towards reduced demyelination in mice treated with BNPs based on the LFB-PAS staining of the spinal cord sections. This supports our previous conclusions that BNPs are suitable for the treatment of EAE. 


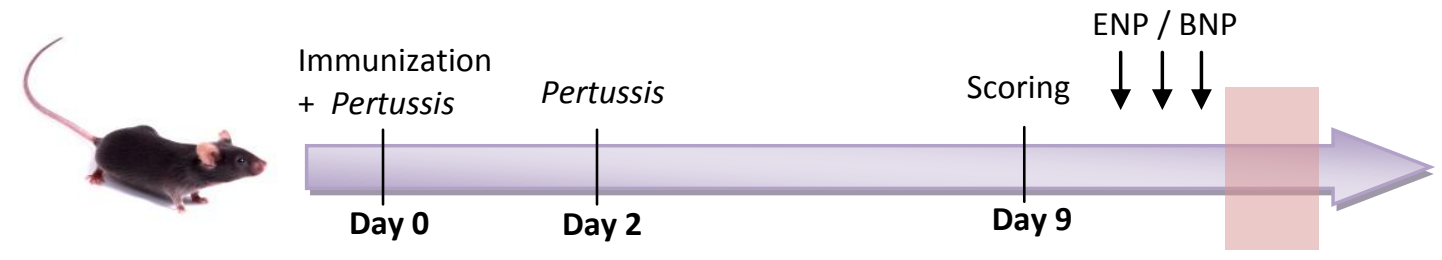

A

ENP

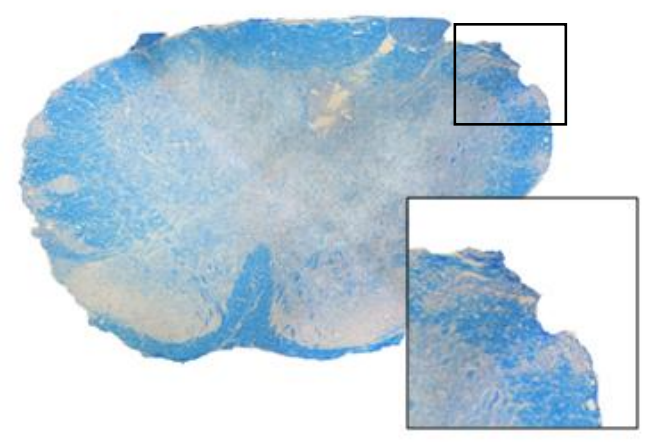

B

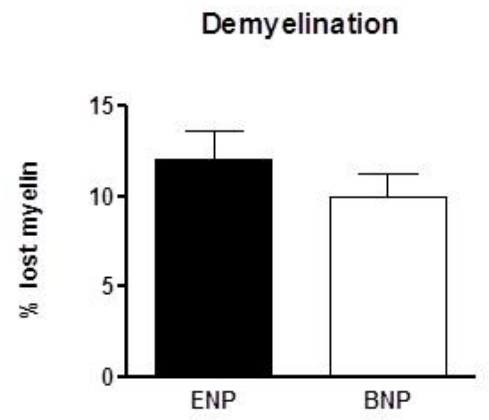

BNP
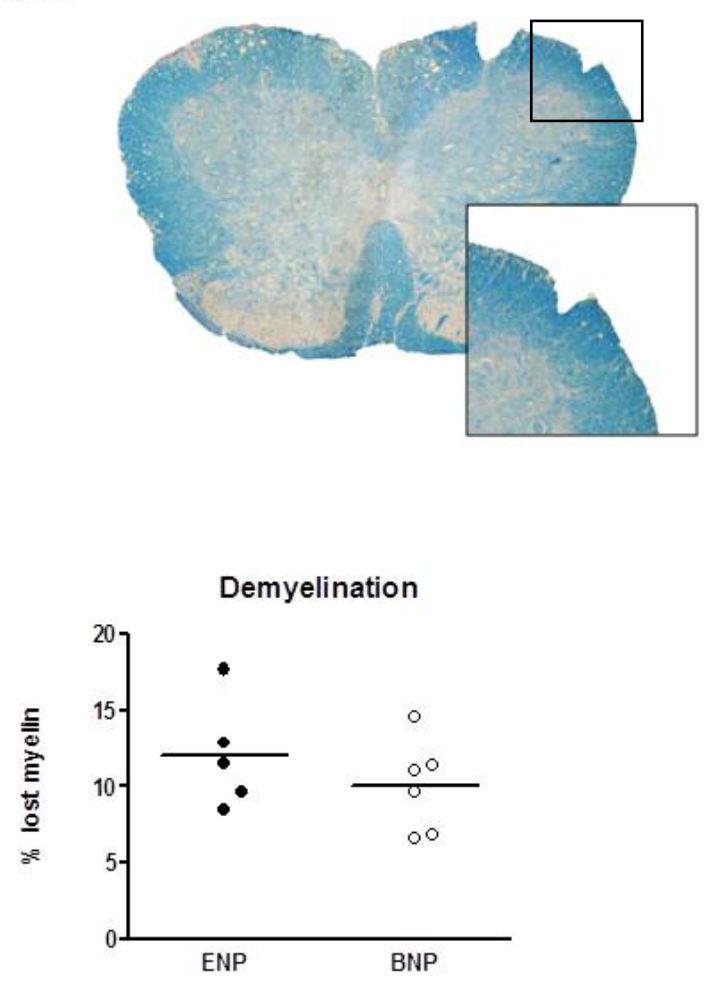

Figure 3.7 | BNPs improve myelin pathology during EAE. C57BL/6 wt mice suffering from EAE were treated three times with BNPs on consecutive days once they had reached a clinical score of 2-3 $(n=6)$. Control mice received the same volume of ENPs $(n=5)$. One day after the third injection, the mice were sacrificed and perfused with $4 \%$ PFA. Spinal cords were isolated, embedded in paraffin, $3 \mu \mathrm{m}$ sections prepared, and subjected to LFB-PAS staining (A). Quantitative analysis of the demyelinated areas was made using the ImageJ 1.46r Software (B). Values are depicted as mean \pm SEM. 


\subsubsection{Myeloid cells are essential targets of BNPs in EAE therapy}

In the past years, several publications showed that T cells are major targets of GCs in the treatment of EAE (Wüst et al. 2008; Schweingruber et al. 2014). Nevertheless, alternative formulations might alter the mechanism of action of a drug, and previous data from our group revealed that liposomal encapsulation conferred an alternative cell-type specificity to GCs (Schweingruber et al. 2011). So far BNPs appeared to have a preference for myeloid cells over T cells. Therefore, we wanted to find out which cell type was the target of these nanoparticles in the context of EAE therapy.

First, we performed immunohistochemical characterization of the CNS infiltrates in immunized mice treated with ENPs or BNPs. The histological study did not reveal changes in T cell infiltration into the spinal cord after BNP treatment, however, the area occupied by macrophages was significantly reduced in the group of mice treated with BNPs as compared to the control mice receiving ENPs (Figure 3.8). These results provide first evidence that nanoparticles act via myeloid cells in the therapy of EAE, as hypothesized.

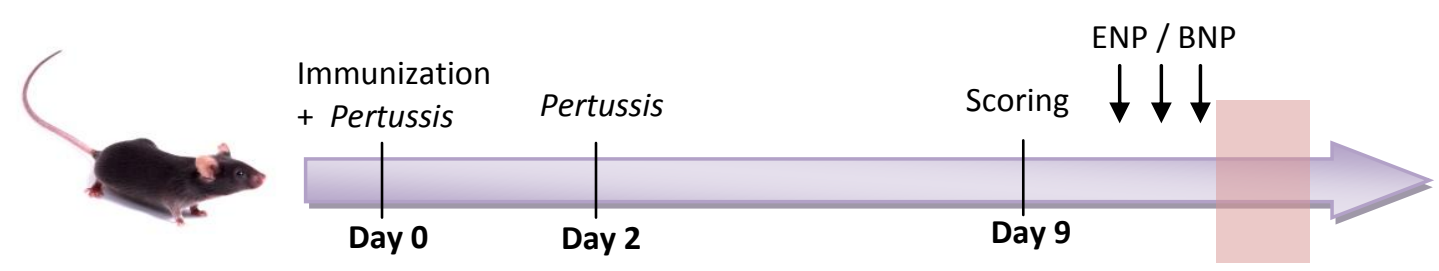

A CD3
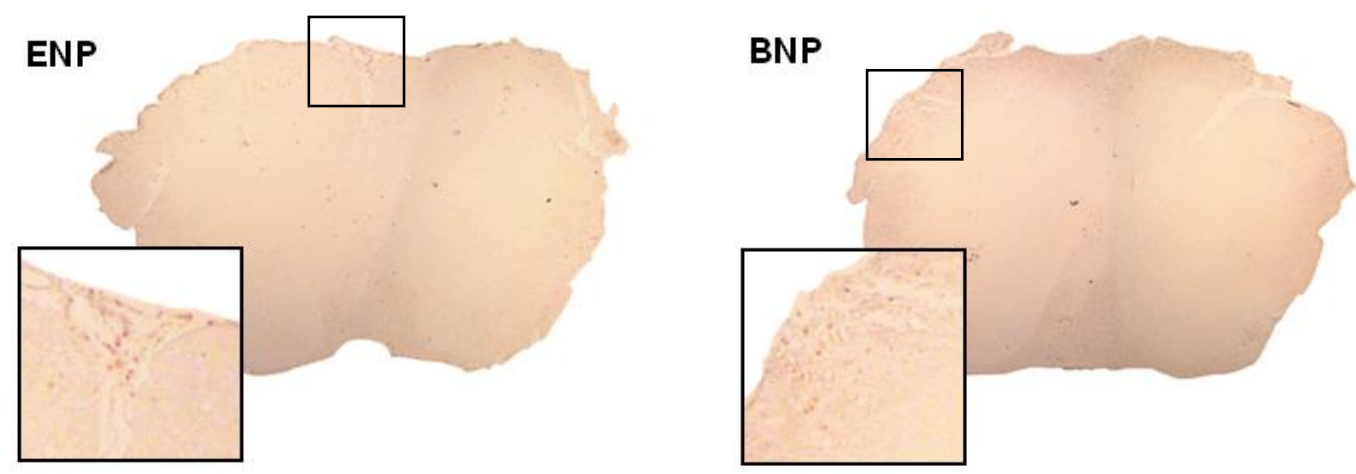

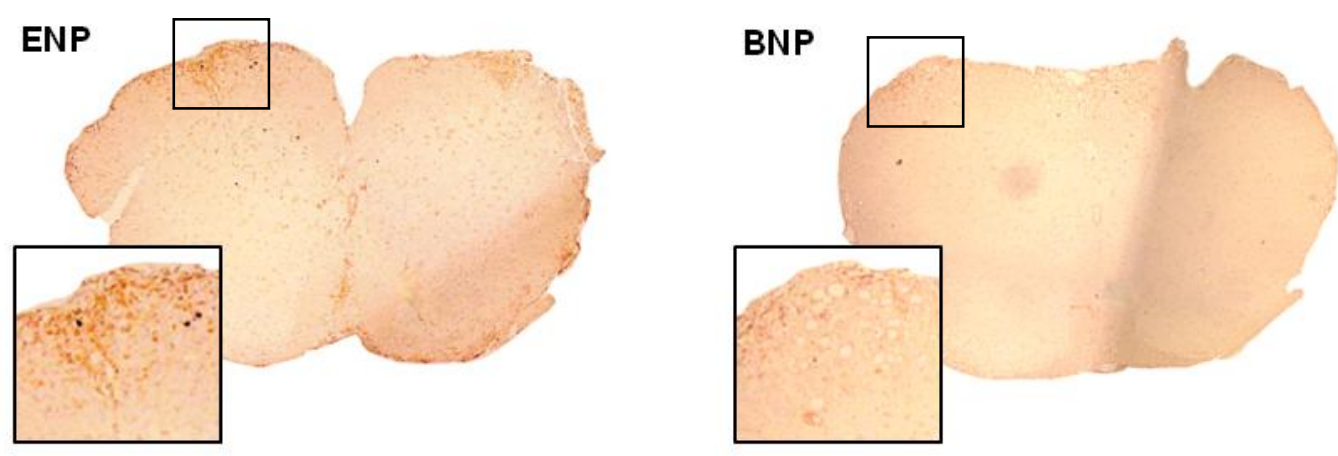

C
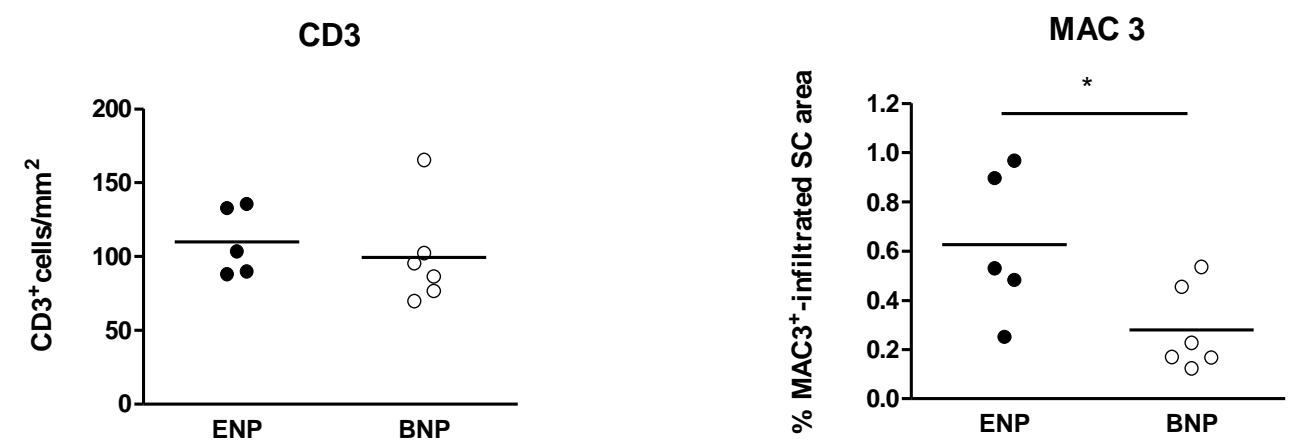

Figure 3.8 | BNPs reduce macrophage infiltration during EAE therapy. C57BL/6 wt mice suffering from EAE were treated three times on consecutive days with BNPs once they reached a clinical score of 2-3 $(n=6)$. Control mice received the same amount of ENPs $(n=5)$. One day after the last injection, the mice were sacrificed and perfused with 4\% PFA. Spinal cords were isolated, embedded in paraffin, and $3 \mu \mathrm{m}$ sections were stained with monoclonal Abs against $\mathrm{CD} 3$ (A) or MAC3 (B). Counting of $\mathrm{CD}^{+}$cells and quantification of macrophage infiltration were performed with the ImageJ $1.46 \mathrm{r}$ software (C). Statistical significance was calculated using the unpaired Student's t-test and values are depicted as mean $\pm \mathrm{SEM} ;{ }^{*}, \mathrm{p}<0.05$.

In the previous in vitro and in vivo experiments it had been found that the presence of the GR was necessary for the immunomodulatory effects of the BNPs, regardless of the cell type (see Figure 3.4, Figure 3.5). Based on this observation we hypothesized that the therapeutic effect of the BNPs should be abrogated when the GR is deleted from any cell type being essential for the therapeutic efficacy of this treatment. 
To address this issue, EAE was induced in several cell type-specific GR knock-out mice. GR ${ }^{\text {lck }}$ mice lack the GR specifically in T cells; GR ${ }^{l y s M}$ are GR-deficient in myeloid cells; $G R^{\text {lck/lysM }}$ have a GR deletion simultaneously in T cells and macrophages; and GR ${ }^{\mathrm{SLCO1C1}}$ carry an inducible GR knock-out construct in endothelial cells of the BBB. GR ${ }^{\mathrm{fl}}$ littermates serve as controls in each case. Once the mice reached a clinical score of 2-3, they were treated with $10 \mathrm{mg} / \mathrm{kg}$ BNPs or the same amount of the empty vehicle according to our standard protocol (see Figure 3.6). The deletion of the GR in T cells did not affect the capacity of the BNPs to improve EAE, suggesting that they were not essential targets in this context (Figure 3.9 A). In contrast, $\mathrm{GR}^{\mathrm{lysM}}$ mice not only presented with a more severe disease course, but were also completely refractory to the treatment with BNPs (Figure 3.9 B). In line with these findings, the double knock-out mice neither responded to BNP treatment (Figure 3.9 C). Finally, GR ${ }^{\text {SLCO1C1 }}$ mice responded to the therapy with BNPs, confirming that modulation of brain endothelial cells was dispensable for the beneficial effect of the GC-nanoparticles (Figure 3.9 D).

A

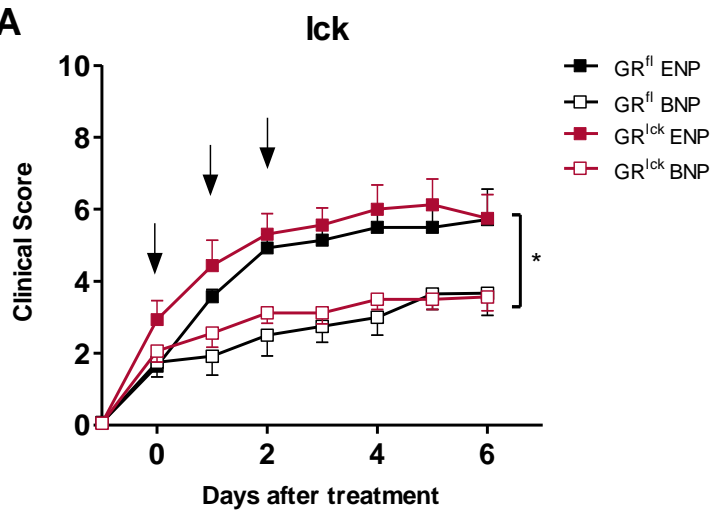

C

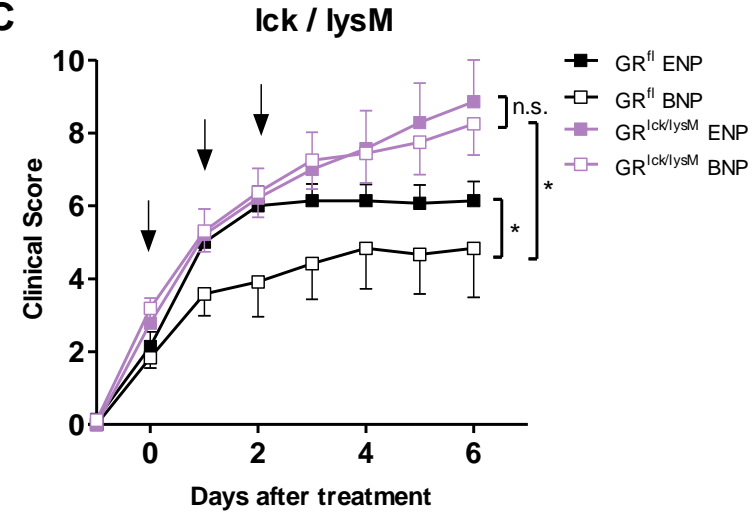

B

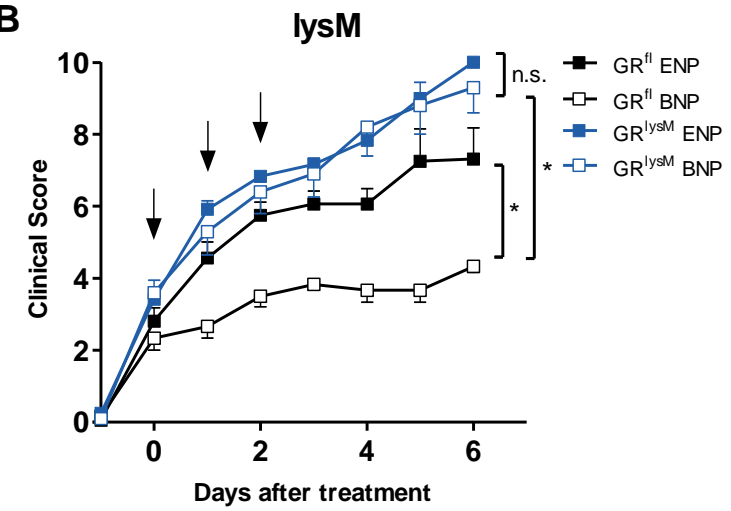

D

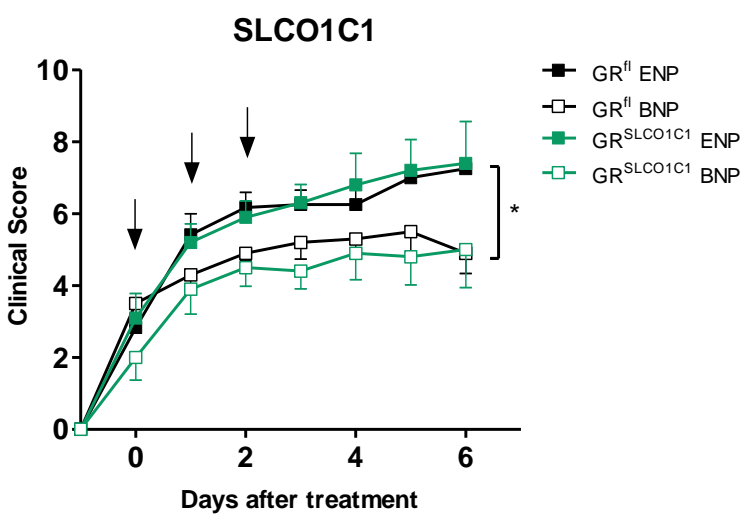


Figure 3.9 | Myeloid cells are essential targets of BNPs in the treatment of EAE. EAE was induced by active immunization with $M_{35-55}$ in four different $G R$ knock out mouse strains: $(A) G R^{\text {Ick }}(n=7,6,8,8),(B)$ $G R^{\text {lysM }}(n=8,3,6,5),(C) G R^{\text {Ick/lysM }}(n=7,6,7,8)$ and (D) $G R^{\text {SLO1C1 }}(n=6,5,5,5) . G R^{f 1}$ littermates were used as controls in each experiment. $10 \mathrm{mg} / \mathrm{kg}$ BNPs and a similar amount of ENPs were applied i.p. once per day for three consecutive days starting once individual mice reached a clinical score of 2-3. The disease severity was evaluated daily for 6 days after the beginning of the treatment. Statistical analysis of the scoring curves was performed using the Mann-Whitney test; values are depicted as mean \pm SEM; (n.s.), p $\geq 0.05 ;{ }^{*}, p<0.05$.

These data clearly demonstrate that BNPs act mainly via modulation of myeloid cells in the treatment of EAE, whereas T cells and brain endothelial cells play only a minor role for their therapeutic efficacy.

\subsubsection{BNPs and Dex act synergistically in the treatment of EAE}

This study so far demonstrated that the use of the $\mathrm{ZrO}_{2}$ nanoparticles directed GCs to the myeloid compartment, while conventional therapies using free GCs mainly target $\mathrm{T}$ cells. Since both cell types participate in EAE progression, a combination of both delivery methods might cause a synergy via targeting distinct cell types, thereby potentiating the antiinflammatory properties of GCs even at lower drug concentrations.

In order to explore this possibility, C57BL/6 wt mice suffering from EAE were treated with suboptimal doses of Dex or BNPs, and an additional group received a combination of both with the total amount of GCs being equal to the individual treatments. As observed previously (see Figure 3.6 B), a suboptimal dose of BNPs did not achieve therapeutic efficacy. However, mice injected with suboptimal doses of both BNPs and Dex presented with a slightly, but significantly, milder EAE than the mice treated with Dex alone. This supports our hypothesis regarding a synergy between BNPs and free Dex. 


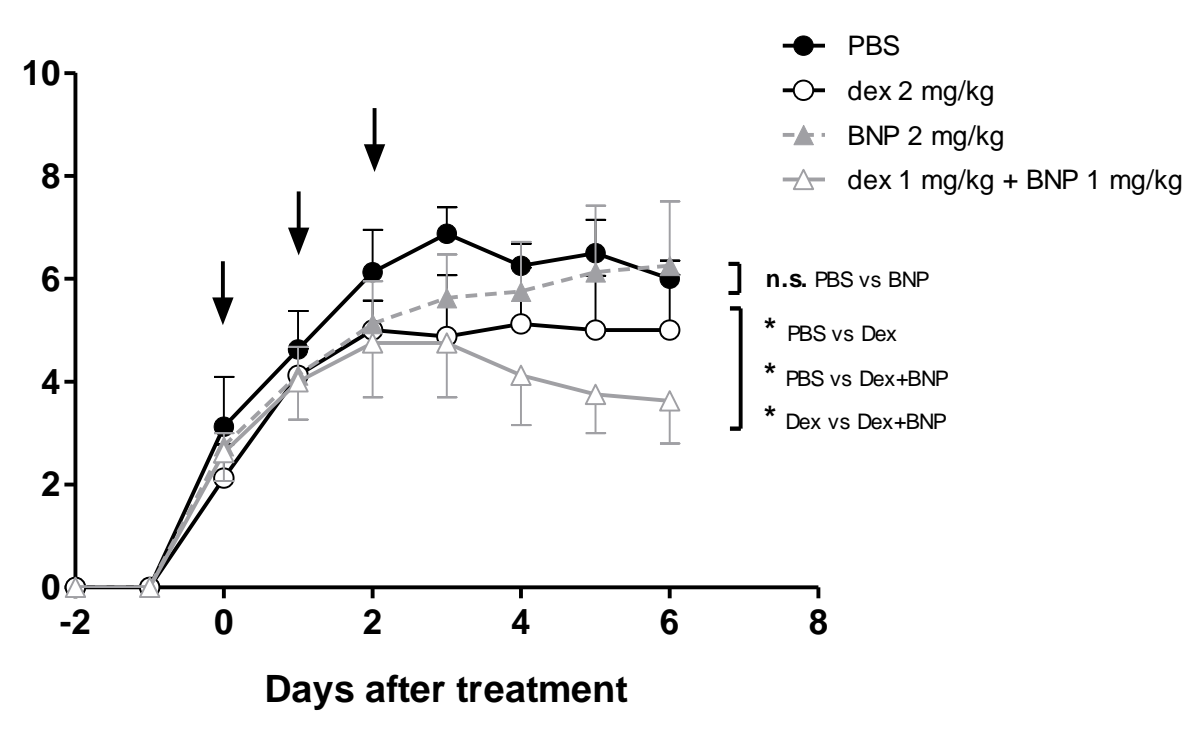

Figure 3.10 | Combined treatment with BNPs and Dex synergistically improves EAE. EAE was induced by active immunization on $\mathrm{C} 57 \mathrm{BL} / 6$ wt mice with $50 \mu \mathrm{g} \mathrm{MOG}_{35-55}$. When mice reached a disability score $>1$, three consecutive daily injections of PBS $(n=4), 2 \mathrm{mg} / \mathrm{kg}$ Dex $(\mathrm{n}=4), 2 \mathrm{mg} / \mathrm{kg}$ BNPs $(\mathrm{n}=4)$ or $1 \mathrm{mg} / \mathrm{kg}$ Dex +1 $\mathrm{mg} / \mathrm{kg}$ BNPs $(n=4)$ were applied i.p. (black arrows). Statistical analysis of the scoring curves was performed using the Mann-Whitney test. Values are depicted as mean \pm SEM; (n.s.),p $\geq 0.05 ;{ }^{*}, p<0.05$.

\subsubsection{BNPs partially circumvent GC-associated side-effects}

A negative aspect of $G C$ therapies is the frequent occurrence of adverse side effects. (Moghadam-Kia and Werth 2010; Weinstein 2012; Ciriaco et al. 2013; Hunter et al. 2014; Hwang and Weiss 2014). This problem derives from the ubiquitous expression of the GR (Rhen and Cidlowski 2005), that makes GCs important players in numerous homeostatic and metabolic processes. By targeting GCs to the myeloid compartment, we aimed to overcome, at least partially, these side effects. Therefore experiments were set up to determine the effect of BNPs in the context of undesired GC activities. 


\subsubsection{Glucose metabolism}

To find out whether the use BNPs circumvented GC-associated hyperglycemia we treated healthy C57BL/6 wt mice on four consecutive days with $10 \mathrm{mg} / \mathrm{kg}$ Dex, $10 \mathrm{mg} / \mathrm{kg} \mathrm{BNPs}$, or their respective vehicles. One day after the last injection, glucose and insulin levels, as well as the expression of typical gluconeogenetic enzymes in the liver were measured after $\mathrm{O} / \mathrm{N}$ fasting. The increase in blood glucose levels was minimal, presumably due to the moderate dose of the drug (Figure 3.11 A). Consistent with the little increase in glucose, insulin concentrations in the serum were unaltered (Figure 3.11 B). Surprisingly, the nanoparticles appeared to lower insulin levels, regardless of whether they contained BMZ or not. The reason and relevance of this effect is not known. Regarding gluconeogenesis, the expression levels of the three analyzed hepatic enzymes were significantly increased by Dex and BNPS (Figure $3.11 \mathrm{C}$ ).

A

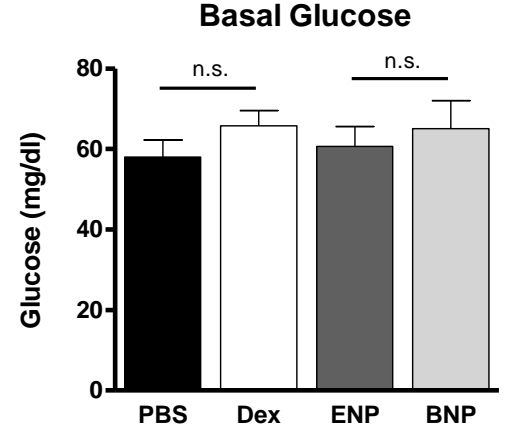

B

Insulin

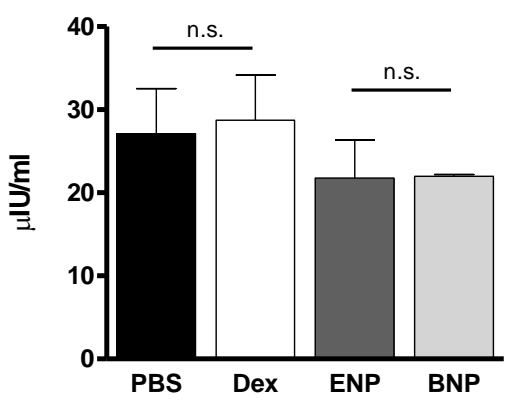

C
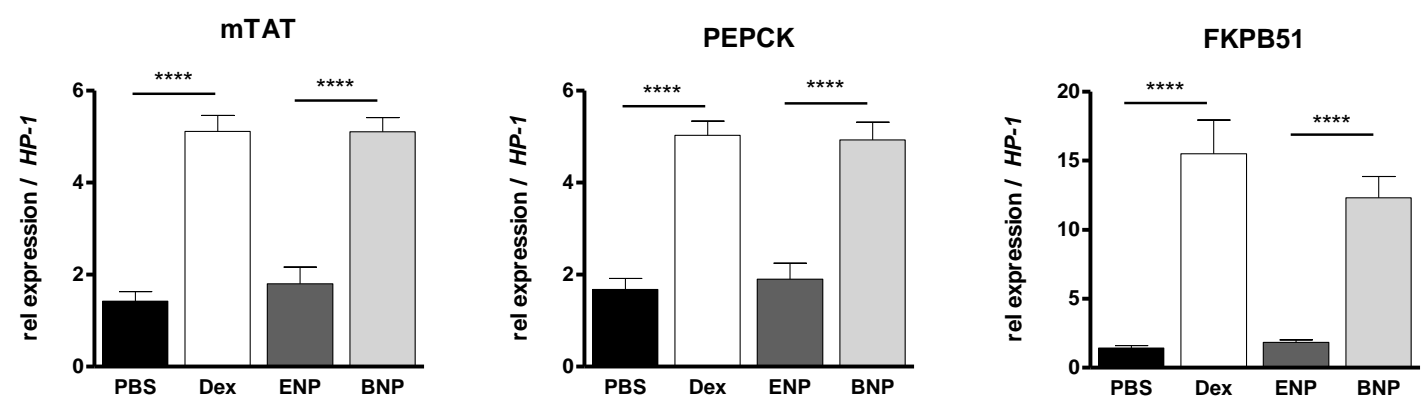
Figure 3.11 | Short treatment with BNPs affects glucose metabolism similarly to Dex. C57BL/6 wt mice were treated i.p. with PBS $(n=12), 10 \mathrm{mg} / \mathrm{kg}$ Dex $(n=13)$, ENPs $(n=10)$ or $10 \mathrm{mg} / \mathrm{kg}$ BNPs $(n=10)$ once per day for four consecutive days. Subsequently, the food pellets were removed O/N. In the morning (9 AM), glucose levels were measured in blood obtained via tail puncture (A). Afterwards, the mice received an additional injection of the corresponding drug, and $2.5 \mathrm{~h}$ later blood serum and liver samples were collected. Insulin levels were determined in serum samples by ELISA (B). The liver samples were used to extract RNA which, after reverse transcription, was used to analyze the expression levels of gluconeogenetic enzymes by qRT-PCR. Expression of the housekeeping gene HP-1 was used as reference (C). Values were analyzed using the unpaired Student's t-test and are depicted as mean \pm SEM; $* * * *, p<0.0001$.

Since hyperglycemia could hardly be observed, even after application of free GCs, no definite conclusions about the properties of BNPs in this respect could be drawn at this point. To address this, further experiments with modified experimental protocols will be performed in the future.

\subsubsection{Muscle wasting}

Another complication observed after GC therapy is skeletal muscle atrophy. To investigate this effect of BNPs, changes in muscle mass were measured in mice treated with Dex or BNPs for four days. On the day after the last injection, the hind limb muscles of the mice were removed, weighted and the values were normalized to the total body weight. Despite the short duration of the corticoid therapy, a slight but non-significant decrease in tibialis anterior and gastrocnemius mass was found in the Dex-treated mice, and to a minor extent also in BNP-treated animals (Figure 3.12). As for glucose and insulin levels, the GC dose used in this experimental setup appears to be too low to induce meaningful metabolic side-effects in mice. 
Tibialis anterior

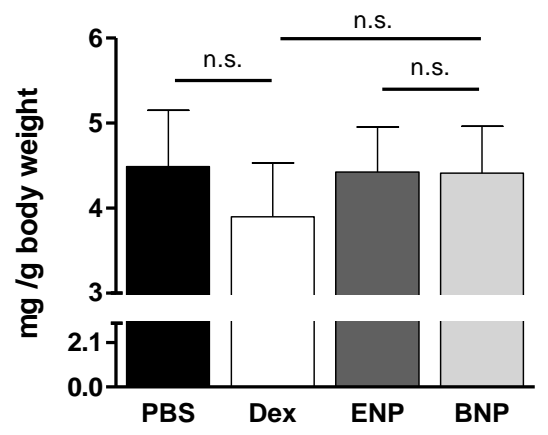

Gastrocnemius

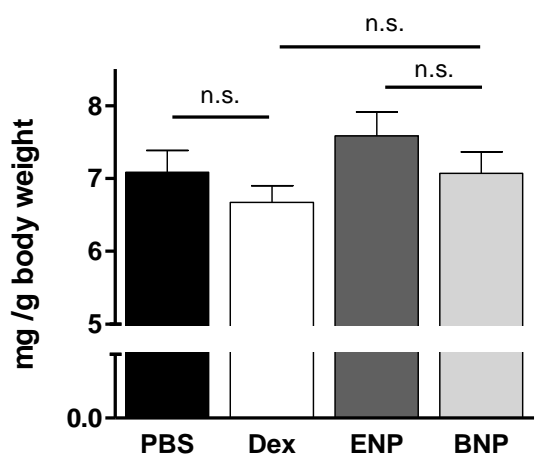

Figure 3.12 | Short treatment with BNP hardly affects muscle wasting. C57BL/6 wt mice were treated i.p. with PBS $(n=10), 10 \mathrm{mg} / \mathrm{kg}$ Dex $(n=10)$, ENPs $(n=10)$ and $10 \mathrm{mg} / \mathrm{kg}$ BNPs $(n=10)$ once per day for four consecutive days. The mice were weighted and sacrificed on the day after the last treatment. Skin was removed from the hind limbs to expose the muscle, and tibialis anterior and gastrocnemius were dissected. The weight of the muscles was determined and referred to the total body weight in each individual mouse. Values were analyzed using the unpaired Student's t-test and are depicted as mean \pm SEM; (n.s.), $p \geq 0.05$.

\subsubsection{Stomach emptying}

It has been demonstrated that GC treatment alters NO metabolism in the stomach, slowing down gastric motility (Reichardt et al. 2014). As a consequence, stomach emptying is impaired resulting in an increased weight of the filled stomach. To address this issue, mice were treated with PBS, $10 \mathrm{mg} / \mathrm{kg}$ Dex, ENPs or $10 \mathrm{mg} / \mathrm{kg}$ BNPs and the weight of the stomach was determined. In both groups receiving GCs, a small but non-significant increase in stomach weight was observed (Figure 3.13). This preliminary data indicates that BNP therapy might impair stomach emptying in a similar manner as free Dex and therefore the use of the nanoparticles might not circumvent this GC-associated side-effect. 


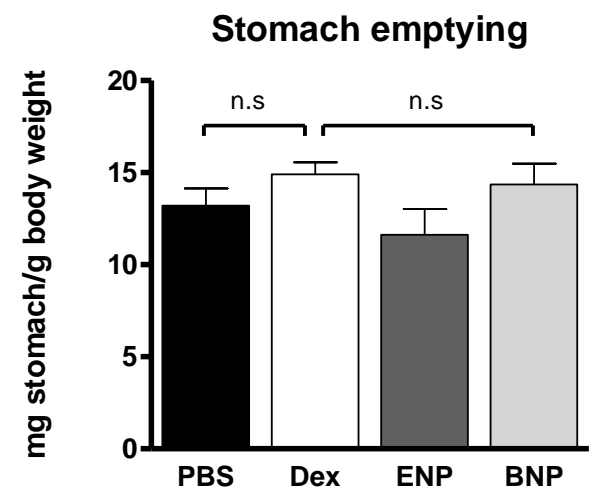

Figure 3.13 | Short treatment with BNPs and Dex has a mild effect on gastric emptying. C57BL/6 wt mice were treated i.p. with PBS $(n=5), 10 \mathrm{mg} / \mathrm{kg}$ Dex $(n=5)$, ENPs $(n=5)$ and $10 \mathrm{mg} / \mathrm{kg}$ BNPs $(n=5)$ once per day for four consecutive days. One day after the last treatment the mice were weighted and sacrificed. The full stomach was dissected, weighted and the obtained values were normalized to the total body weight in each individual mouse. Values were analyzed using the unpaired Student's t-test and are depicted as mean \pm SEM; (n.s.), $p \geq 0.05$.

\subsubsection{Bone re-sorption}

An important side effect of GC therapy is the decrease in bone mineral density. To evaluate whether BNPs improved this symptom or not, osteocalcin was used as a surrogate marker of GC-induced osteoporosis, since it is quickly reduced after GC application (Leclerc et al. 2005). The concentrations of osteocalcin in the serum of mice treated with PBS, $10 \mathrm{mg} / \mathrm{kg}$ Dex, ENPs or $10 \mathrm{mg} / \mathrm{kg}$ BNPs for four consecutive days were determined by ELISA. With this treatment protocol, no significant changes in osteocalcin levels could be detected between any of the experimental groups. 


\section{Osteocalcin}

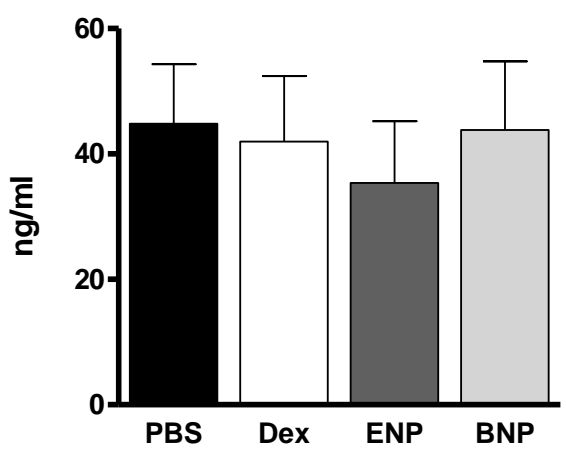

Figure 3.14 | Short treatment with BNPs does not affect the osteoporosis marker osteocalcin. Blood was obtained via heart puncture from C57BL/6 wt mice receiving a 4-day treatment with PBS ( $n=10), 10 \mathrm{mg} / \mathrm{kg}$ Dex $(n=10)$, ENPs or $10 \mathrm{mg} / \mathrm{kg}$ BNPs $(n=10)$. Serum was separated in a STT Microtainer ${ }^{\mathrm{TM}}$ tubes by centrifugation. The levels of osteocalcin were determined on the serum samples by ELISA. Values were analyzed using the unpaired Student's t-test and are depicted as mean \pm SEM; (n.s.), $p \geq 0.05$.

Collectively, our data indicates that the experimental protocol used in the analysis of GCassociated side symptoms needs to be optimized in order to obtain reliable information about the potential of the BNPs to avoid the appearance of unwanted effects. 


\subsection{Role of the MR in myeloid cells in EAE}

The previous results of this thesis confirmed the crucial role of myeloid cells for the response to GCs in EAE, and showed that this modulation is mediated by the GR. However, the GR is not the only receptor sensitive to GCs in this cell compartment. The MR is also expressed in myeloid cells and is known to promote a pro-inflammatory state in mouse peritoneal macrophages after GC binding (Usher et al. 2010). Hence, we assumed that the deletion of the MR in myeloid cells might potentiate the anti-inflammatory activities of GCs via the GR. Therefore, the aim of the second part of this thesis was to characterize the CNS autoimmune responses in $\mathrm{MR}^{\mathrm{lys} M}$ mice, a knock-out mouse strain harboring a MR deficiency specifically in the myeloid compartment.

\subsubsection{MR ${ }^{\mathrm{lys} M}$ mice develop a milder EAE disease}

This project is based on the previous work of $\mathrm{Li}$ and Schweingruber, two former colleagues of the Reichardt's group, who performed extensive in vitro analyses of macrophages derived from $M R^{l y s M}$ mice. MR-deficient macrophages expressed lower levels of pro-inflammatory genes compared to cells from $\mathrm{MR}^{\mathrm{fl}}$ controls (Li 2013). In line with these results, their capacity to secrete TNF $\alpha$ and NO was diminished as well. Analysis of EAE in MR ${ }^{\mathrm{lysM}}$ mice showed a milder disease course (Figure 3.15), which was reproducible in our current experiments. Furthermore, both peripheral and CNS-infiltrating macrophages from the affected mice adopted a M2 phenotype, confirming a correlation between EAE severity and myeloid cell phenotype (Li 2013). 


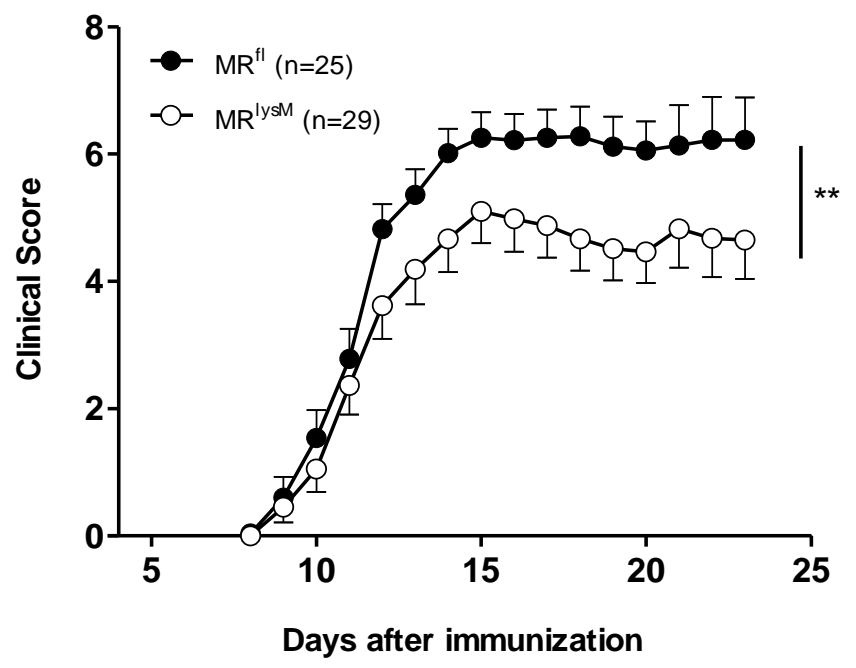

Figure 3.15 | $\mid R^{l y s M}$ mice develop a milder EAE phenotype. EAE was induced in $M R^{f l}$ and $M R^{l y s M}$ mice by active immunization with $50 \mu \mathrm{g}$ of $\mathrm{MOG}_{35-55}$ emulsified in CFA. Mice were weighted and scored daily from day 9 post-immunization on. (A) Cumulative disease curve as determined in experiments performed by Li and Schweingruber (Li 2013). Statistical analysis was performed using the Mann-Whitney test; values are depicted as mean $\pm \mathrm{SEM} ; * *, \mathrm{p}<0.01$.

The aim of this part of my thesis was to continue and complete the characterization of EAE in $M R^{\text {lysM }}$ mice. Since the clinical signs of disability in EAE generally mirror neuronal pathology, we aimed to corroborate the milder disease course observed in the $M R^{\text {lysM }}$ group via histological analysis of the spinal cord in those mice. These experiments were performed in collaboration with Li and Schweingruber, and the preliminary histological data was already included in the thesis of Li (Li 2013).

First, CNS infiltration by immune cells was determined. Once T cells have been primed by antigen-specific APCs in the periphery, they proliferate and acquire effector functions. Several days later, they migrate to the CNS to encounter their cognate antigen again and become re-stimulated, therefore infiltration of antigen-specific T cells is considered a major hallmark of EAE pathogenesis (Schweingruber et al. 2014). The histological analysis of $M R^{\text {lysM }}$ mice revealed that, in spite of the milder disease course in the $M R^{l y s M}$ mice, infiltration by $\mathrm{CD}^{+}$cells into the spinal cord was largely similar in both genotypes (Figure 3.16 A). Besides 
$T$ cell migration, a massive infiltration of monocytes is observed at the onset of EAE and during MS relapses (Huitinga et al. 1990). Once in the CNS, these monocytes differentiate into macrophages that process and present myelin antigens, thereby contributing to the secretion of cytokines and other soluble mediators which cause oligodendroglial death and neuronal damage. Since MR-deletion might affect different features of monocytes/macrophages including migration, the presence of monocytes and macrophages in the CNS was also investigated by IHC using an anti-MAC3 Ab. Indeed, the infiltration of the spinal cord by $\mathrm{MAC3}^{+}$cells in $\mathrm{MR}^{\text {lysM }}$ mice was significantly lower compared to the $\mathrm{MR}^{\mathrm{fl}}$ control animals (Figure 3.16 B).

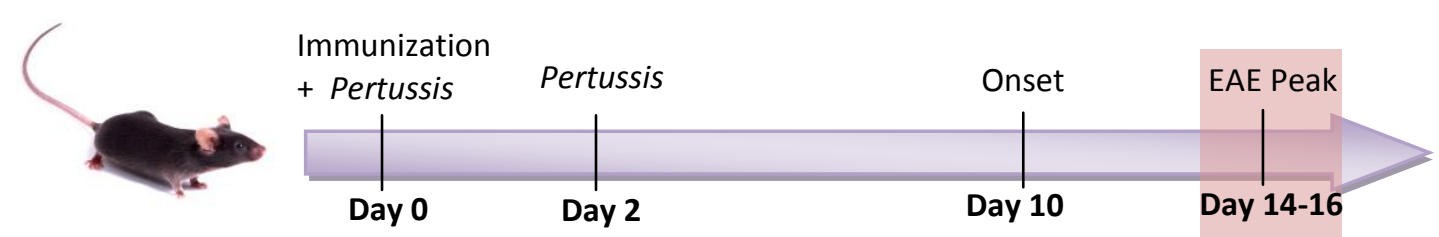

A
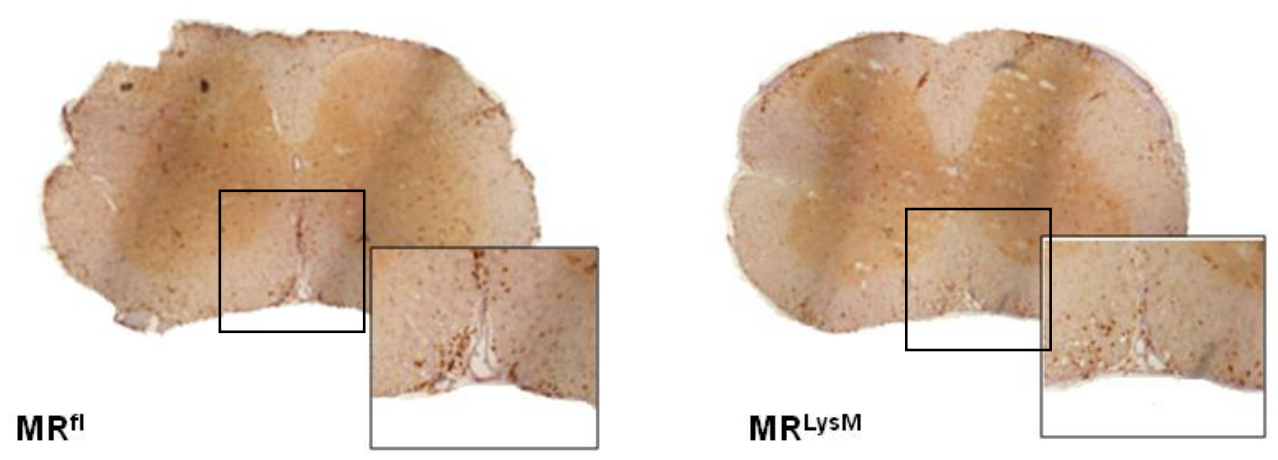

B
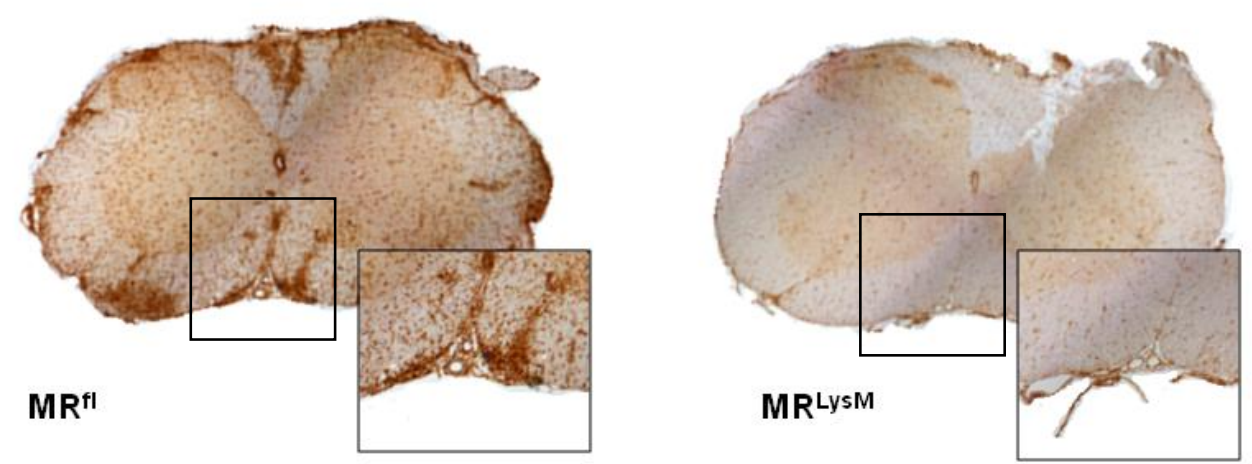
C

$\mathrm{CD3}^{+}$infiltration

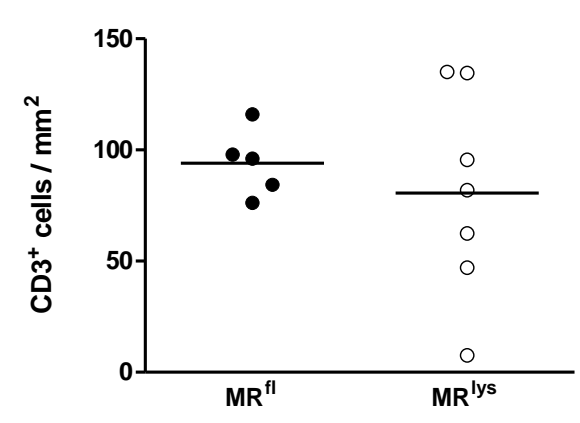

MAC3 ${ }^{+}$infiltration

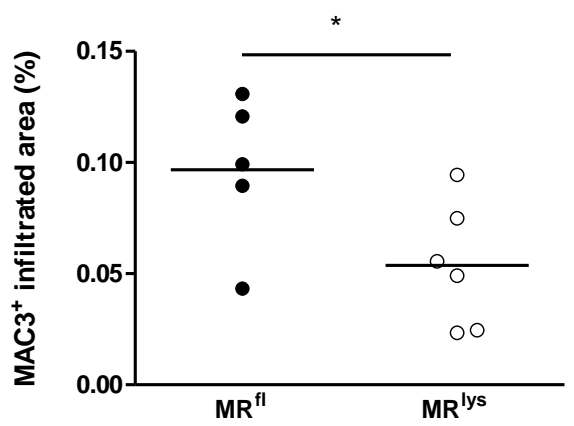

Figure 3.16 | Macrophage infiltration into the spinal cord is reduced in MRlysM mice during EAE. $\mathrm{MR}^{\mathrm{fl}}(\mathrm{n}=5)$ and $M R^{\mathrm{LysM}}(\mathrm{n}=7-6)$ mice were immunized according to our standard protocol (see 2.3.1) and spinal cords were isolated and fixed in PFA at the peak of the disease (in collaboration with Li and Schweingruber). After paraffin embedding, $3 \mu \mathrm{m}$ sections were cut and mounted on microscopic slides. IHC staining was performed with Abs against $\mathrm{CD} 3(\mathbf{A})$ and $\mathrm{MAC} 3(\mathbf{B})$. The number of $\mathrm{CD}^{+}$cells per $\mathrm{mm}^{3}$ of total spinal cord area and the area infiltrated by $\mathrm{MAC3}^{+}$cells in relation to the total white matter area were determined with the help of the ImageJ $1.46 \mathrm{r}$ software (C). Values were analyzed using the unpaired Student's t-test and are depicted as mean \pm SEM; * $\mathrm{p}<0.05$.

Additionally, the extent of neurodegeneration in these mice was evaluated. To this end, histological analysis of demyelination and axonal loss were performed on spinal cord sections from $\mathrm{MR}^{\mathrm{fl}}$ and $\mathrm{MR}^{\mathrm{lysM}}$ mice at the peak of EAE disease. In agreement with the milder EAE score, the average number and size of demyelinated lesions was significantly reduced in the myeloid-specific knock-out mice. Furthermore, axonal density in $\mathrm{MR}^{\mathrm{lysM}}$ mice was partially preserved (Figure 3.17).

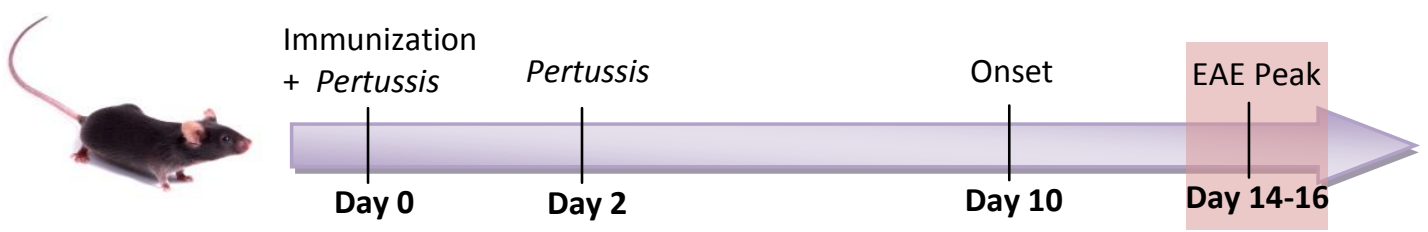


A

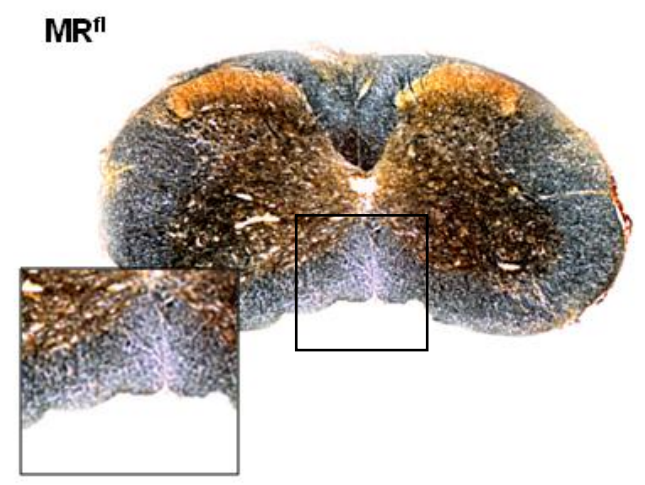

B

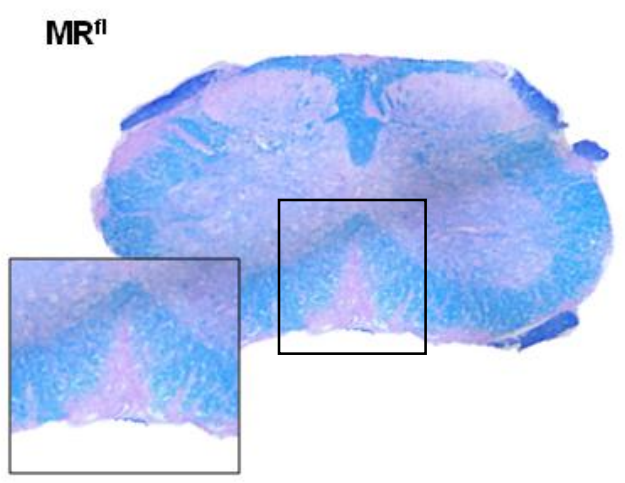

C

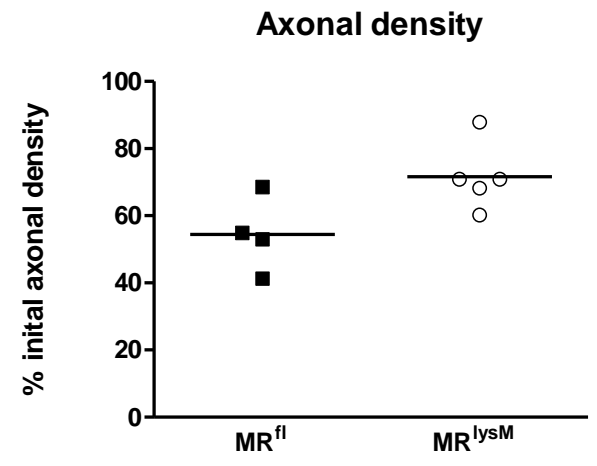

MR

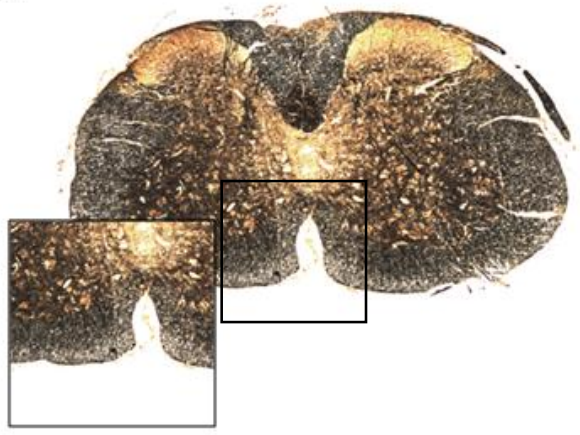

MR ${ }^{\text {lysm }}$

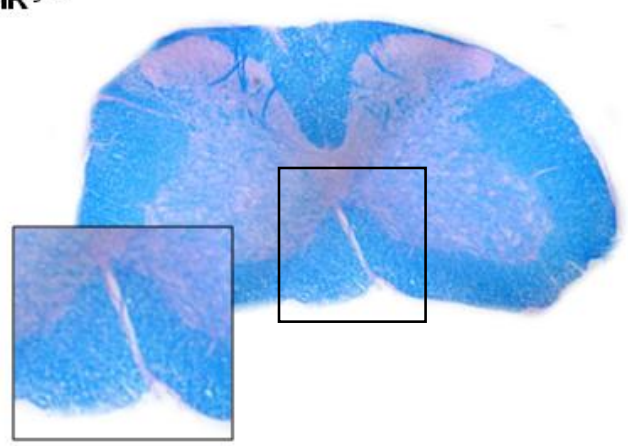

Demyelination

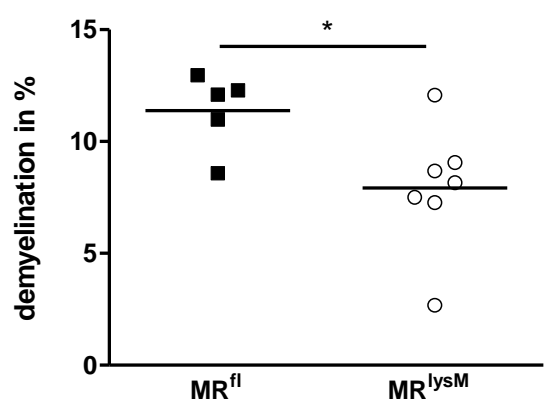

Figure 3.17 | Demyelination and axonal damage in MR ${ }^{\text {lysM }}$ mice during EAE are not as severe as in MR $^{\mathrm{fl}}$ mice. $M R^{\mathrm{fl}}(\mathrm{n}=5-4)$ and $M R^{\mathrm{LySM}}(\mathrm{n}=7-5)$ mice were immunized according to our standard protocol (see 2.3.1) and spinal cords were isolated and fixed in PFA at the peak of the disease (in collaboration with Li and Schweingruber). After paraffin embedding, $3 \mu \mathrm{m}$ sections were cut and mounted on microscopic slides. Bielschowsky silver staining was performed to visualize axonal fibers (A), and myelin was stained according to the LFB/PAS protocol (B). Quantification of the axonal densities and the percentage of myelin lost were carried 
out with the Image $1.46 \mathrm{r}$ software (C). Values were analyzed with the unpaired Student's t-test and are depicted as mean \pm SEM; $*, p<0.05$.

Taken together, deletion of the MR in myeloid cells markedly ameliorates EAE in terms of clinical symptoms, monocyte infiltration into the spinal cord and demyelination.

\subsubsection{MR deletion alters the activation state of circulating and CNS- infiltrating monocytes}

In search of mechanisms that could explain the reduced presence of monocytes and macrophages in the CNS of MR-deficient mice, the activation state of peripheral and CNSinfiltrating monocytes was analyzed at the onset and peak of EAE. Blood circulating monocytes were defined as CD11 ${ }^{+}$Ly6G cells, whereas in the CNS they were distinguished from other resident myeloid cells on the basis of their $\mathrm{CD} 11 \mathrm{~b}^{+} \mathrm{CD} 45^{\text {high }}$ phenotype. The numbers of inflammatory monocytes, which can be distinguished from resting ones due to an increased expression of Ly6C on their surface, were determined by FACS analysis using a monoclonal Ab against Ly6C (Figure 3.18). Based on this systematic we observed that the percentage of inflammatory monocytes in blood and spinal cord was significantly reduced in mutant mice. Thus, a reduction in the number of activated monocytes could be one of factors contributing to the ameliorated EAE observed in $M R^{\mathrm{lysM}}$ mice.

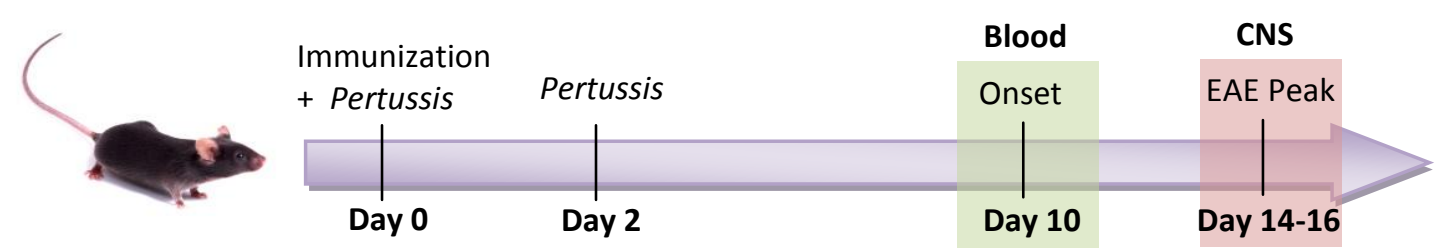


A

Blood circulating monocytes
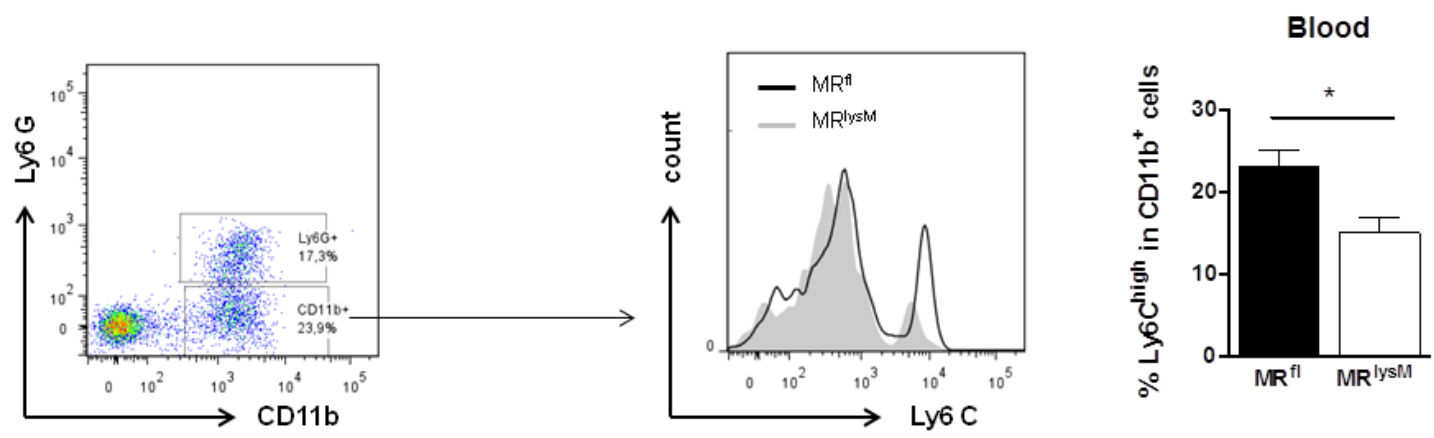

B

\section{CNS infiltrating monocytes}
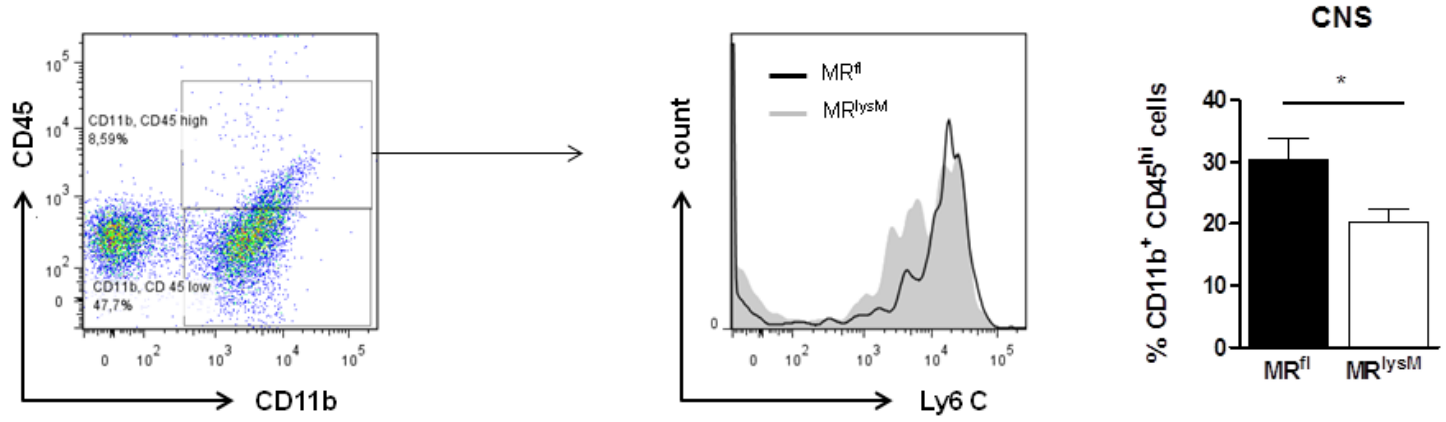

Figure 3.18 | The percentage of inflammatory monocytes in blood and spinal cord of MR ${ }^{l y s M}$ mice is reduced during EAE. EAE was induced according to our standard protocol (see 2.3.1) in $\mathrm{MR}^{\mathrm{fl}}$ and $\mathrm{MR}^{\mathrm{IysM}}$ mice. (A) At day 10 after immunization, the mice were sacrificed and blood was collected in Alsevers solution to avoid clotting $(n=6,4)$. The cells were stained with Abs against CD11b, Ly6C and Ly6G, treated with Optilyse to remove erythrocytes and analyzed $2 \mathrm{~h}$ later by FACS. Inflammatory monocytes were defined according to the systematic of Perlmann and colleagues (Rose et al. 2012). Ly6G $^{+}$cells were excluded from the CD $11 \mathrm{~b}^{+}$ population, and then inflammatory monocytes were distinguished from resting monocytes based on their higher Ly6C expression. (B) Spinal cords from the mice with ongoing EAE were obtained at the peak of the disease (day 14-16), and homogenized to separate mononuclear cells on a Percoll gradient $(n=16,15)$. The cells were stained with Abs against CD11b and CD45.2 to differentiate monocytes/macrophages from microglia (Prinz et al. 2011). Subsequently, the monocytes were subdivided into inflammatory and resting ones on the basis of the Ly6C expression levels. (C) The quantification of the data was performed with GraphPad Prism. Values were analyzed using the unpaired Student's t-test and are depicted as mean \pm SEM; ${ }^{*}, \mathrm{p}<0.05$. 


\subsubsection{Monocytes and neutrophils in secondary lymphoid organs are not affected by the MR deletion}

Up to this point, we have found that the MR deletion affected circulating monocytes and infiltrating monocyte/macrophages during EAE. However we had no information about the activated state of macrophages in peripheral lymphoid organs, where they fulfill an important task shaping the environment for T cell priming and differentiation. Therefore, using the same FACS gating strategy as for blood samples (Figure $\mathbf{3 . 1 8} \mathbf{A}$ ), monocyte activation was analyzed in the spleen and the lymph nodes of $M R^{l y s M}$ mice at disease onset. In these organs we could not find changes between knock-out and control mice in the proportion of Ly6C $C^{\text {high }}$ monocytes (Figure 3.19 A).

It has to be taken into account that in $\mathrm{MR}^{\mathrm{lysM}}$ mice the cre recombinase does not only delete floxed genes in $83-98 \%$ of macrophages, but also in nearly $100 \%$ of neutrophils (Clausen et al. 1999). Neutrophils are a major component of the spinal cord infiltrates in C57BL/6 mice, especially in the early phases of the disease (Soulika et al. 2009; Wu et al. 2010). Hence, to exclude any potential alterations in this cell compartment, the proportion of $\mathrm{CD}_{11} \mathrm{~b}^{+}$cells expressing Ly6G was determined in immunized mice at the onset of the disease. The FACS data revealed that the number of $\mathrm{Ly}_{6 \mathrm{G}}{ }^{+}$cells was similar for both genotypes (Figure 3.19 B). We therefore believe that it is very unlikely that neutrophils contribute to the EAE phenotype observed in $\mathrm{MR}^{\mathrm{lysM}}$ mice.

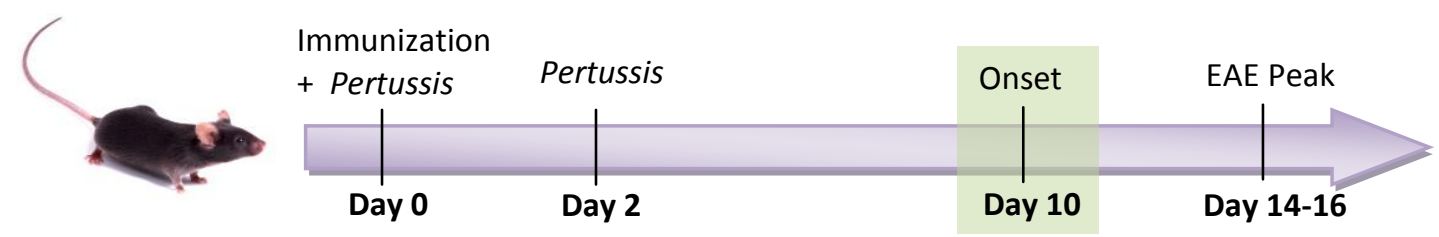


A

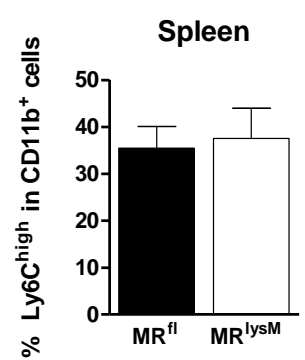

Inflammatory Monocytes

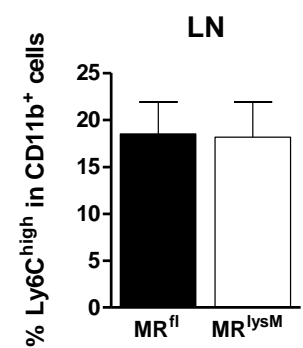

B

Neutrophils

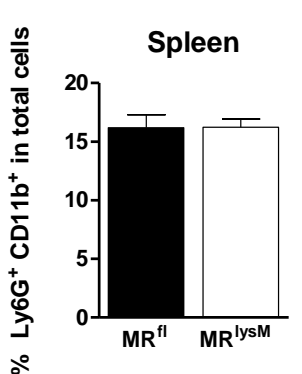

LN

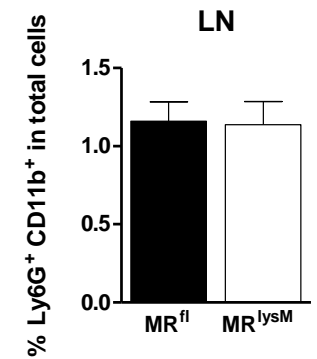

Figure 3.19 | Inflammatory monocytes and neutrophils are unaffected in secondary lymphoid organs of MRlysM mice during EAE. Spleens and lymph nodes from $M R^{f l}(n=9)$ and $M R^{l y s M}(n=7)$ mice were analyzed at day 10 after EAE induction (see 2.3.1). Single-cell suspensions were obtained and stained with monoclonal Abs against CD11b, Ly6G and Ly6C. The percentages of CD11b ${ }^{+}$Ly6C ${ }^{\text {high }}$ cells, corresponding to inflammatory monocytes (A), and $\mathrm{CD} 11 \mathrm{~b}^{+} / \mathrm{Ly}_{6 \mathrm{G}}{ }^{+}$cells, corresponding to neutrophils (B), were determined by FACS. Values were analyzed using the unpaired Student's t-test and are depicted as mean \pm SEM.

\subsubsection{T cell interactions with MRlysM macrophages}

It is generally accepted that MS and EAE are $T$ cell-driven diseases. In the $M R^{1 / 5 \mathrm{MM}}$ mouse model $T$ cells neither express Cre nor the MR, thus there should not be any direct effect of the MR deficiency on T cells. Nevertheless, macrophages present antigens and secrete cytokines that influence T cell differentiation, participating both in the activation of T cells in the periphery and their re-activation in the CNS. Therefore, MR deletion from myeloid cells might impact EAE indirectly via this mechanism as well. To test this hypothesis the interaction between $T$ cells and $M R^{I y s M}$ myeloid cells was investigated. 


\subsubsection{The cytokine secretion profile is altered in MR-deficient BMDMs}

Soluble factors released by macrophages strongly influence T cell differentiation. Hence, we analyzed selected cytokines secreted by BMDMs obtained from $\mathrm{MR}^{\mathrm{fl}}$ and $\mathrm{MR}^{\mathrm{lys} M}$ mice. Following stimulation with IFNy and LPS, the cytokine profile of the activated MR ${ }^{\text {lysM }}$ BMDMs was shifted towards the M2 phenotype (Figure 3.20). More specifically, mutant BMDMs secreted less TNF $\alpha$ and IL-6, considered to be pro-inflammatory, but higher amounts of the regulatory cytokine IL-10. In the absence of IFNy/LPS stimulation, all three cytokines were barely detectable in the supernatant (data not shown).
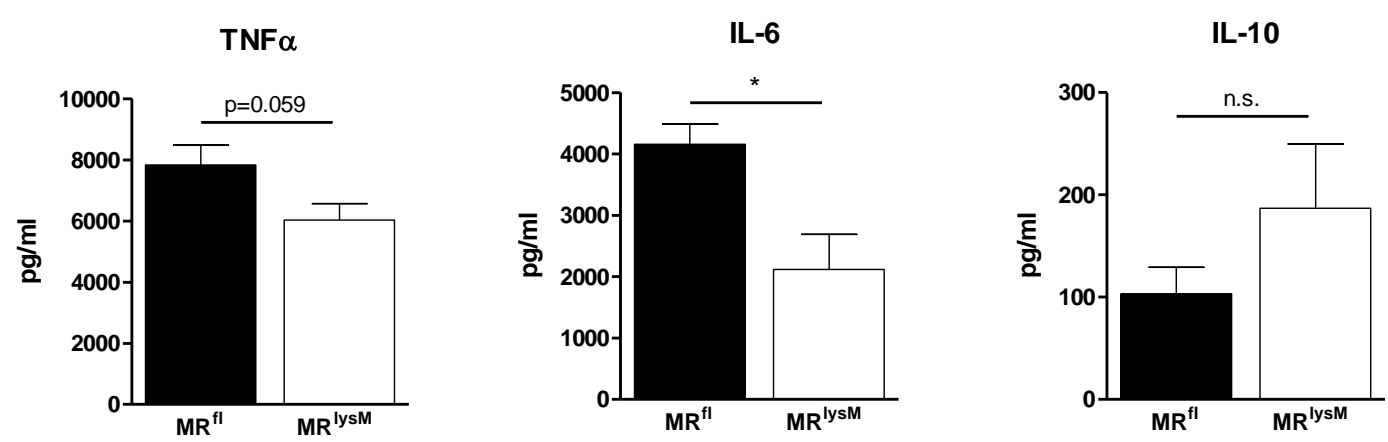

Figure 3.20 | MR-deficient BMDMs secrete less M1 cytokines, whereas M2 cytokine release is increased. $B M D M s$ were generated from $M^{f l}(n=6)$ and $M R^{l y s M}(n=6)$ mice and seeded in 96-well plates at a concentration of $1 \cdot 10^{5}$ cells/well. Then, they were stimulated for $24 \mathrm{~h}$ with $20 \mathrm{ng} / \mathrm{ml} \mathrm{LPS}$ and $50 \mathrm{ng} / \mathrm{ml} \mathrm{IFNy}$. The concentration of TNF $\alpha$, IL- 6 and IL-10 in the supernantant was determined by CBA. Values were analyzed using the unpaired Student's t-test and are depicted as mean $\pm \mathrm{SEM}$; n.s., $p \geq 0.05 ;{ }^{*}, p<0.05$.

\subsubsection{MR ${ }^{\text {lysM }}$ macrophages are potent APCs in vitro}

It is known that macrophages can act as APCs in different situations. Since the phenotypic changes observed in the MR-deficient BMDMs might alter their capacity to present antigens to $T$ cells and to activate them, this feature was evaluated in vitro. $\mathrm{MR}^{\mathrm{fl}}$ and $\mathrm{MR}^{\mathrm{lysM}} \mathrm{BMDMs}$ were co-cultured with 2D2 T cells in the presence of $\mathrm{MOG}_{35-55}$ and the proliferation rate of the $T$ cells was determined by FACS. BMDMs efficiently stimulated T cell proliferation regardless of the genotype (Figure 3.21 B). 
A
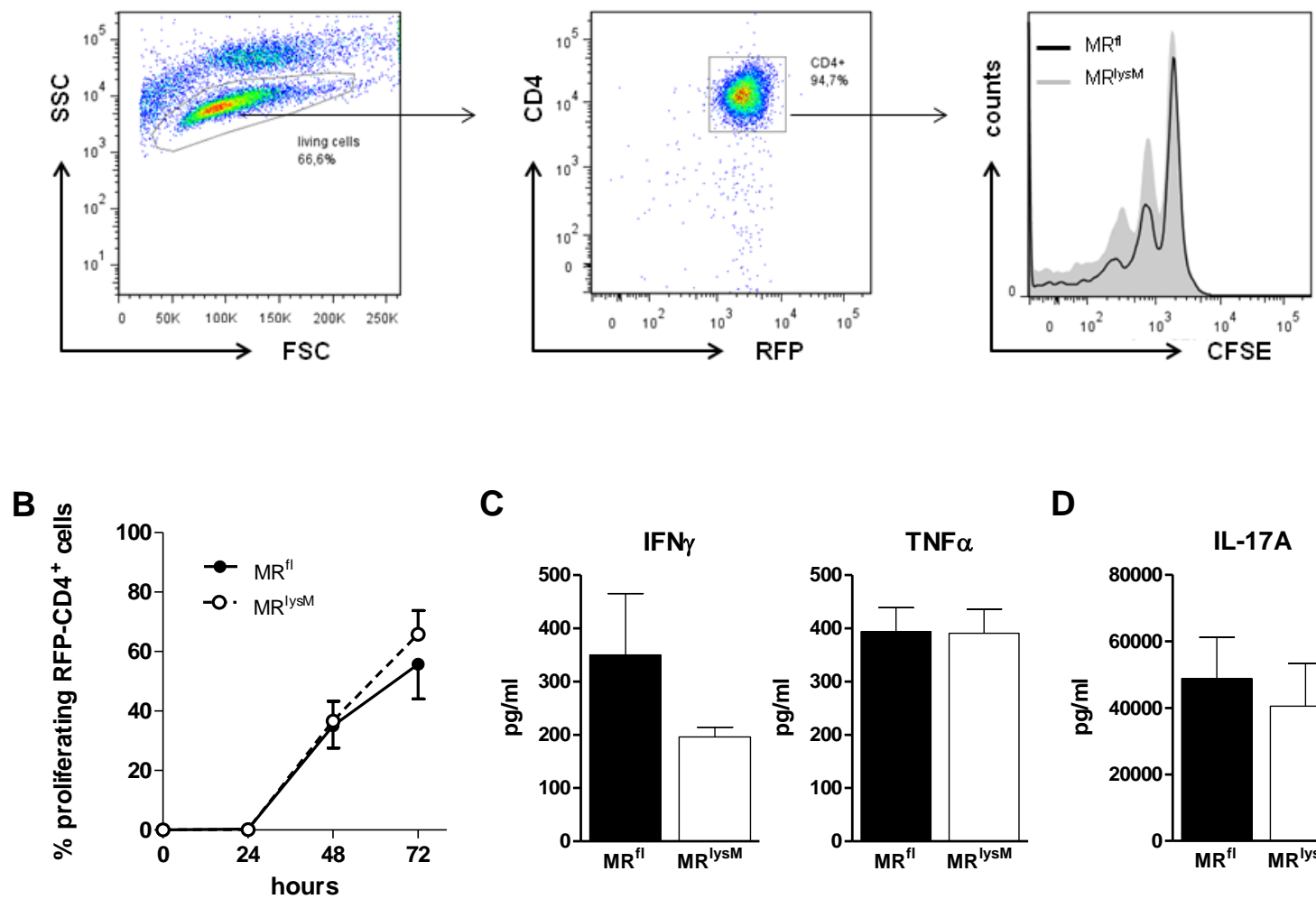

C
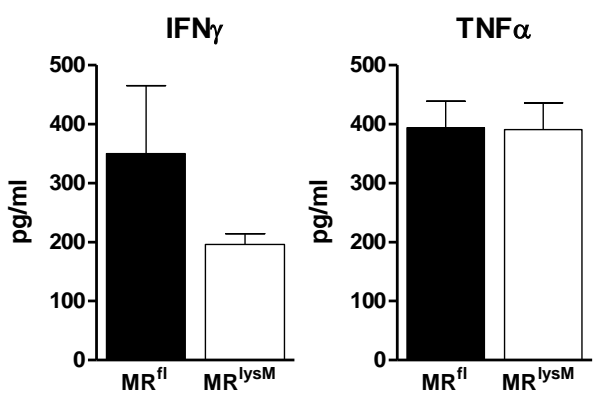

D

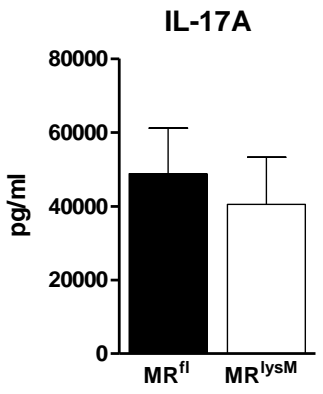

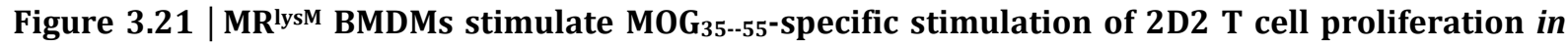
vitro. $B M D M s$ were generated from $\mathrm{MR}^{\mathrm{fl}}(\mathrm{n}=6)$ and $\mathrm{MR}^{\mathrm{lysM}}(\mathrm{n}=6)$ mice. $(A, B) 1 \cdot 10^{5} \mathrm{BMDMs} /$ well were plated in triplicates and the same numbers of CFSE-labeled T cells purified from 2D2 RFP mice were added together with $20 \mu \mathrm{g} / \mathrm{ml} \mathrm{MOG}_{35-55}$. Non-adherent cells were collected after $24 \mathrm{~h}, 48 \mathrm{~h}$ and $72 \mathrm{~h}$, stained with an anti-CD4 Ab and analyzed by FACS. Cell proliferation was determine based on the sequential dilution of the CFSE staining after gating on the $\mathrm{CD}^{+} \mathrm{RFP}^{+}$cell population. (C) After $72 \mathrm{~h}$, the supernatants were collected and analyzed by CBA to determine the levels of selected cytokines. (D) 1.10 $0^{5}$ MDMs generated from the bone marrow of $\mathrm{MR}^{\mathrm{fl}}$ $(n=11)$ and $M R^{l y S M}(n=11)$ mice were plated in 96 well plates and $1 \cdot 10^{5}$ Th17 2D2 cells (kindly provided by Judith Strauß, IMSF Göttingen) were added to the cultures. $72 \mathrm{~h}$ later the supernatants were collected and IL-17A concentrations were determined by ELISA. Values were analyzed using the unpaired Student's t-test and are depicted as mean \pm SEM.

Since the changes in the cytokines produced by the $M R^{\mathrm{lysM}}$ BMDMs might have an effect on $T$ cell activation, the cytokine secretion of different BMDM/2D2 $\mathrm{T}$ cell co-cultures was additionally analyzed by CBA. IFN $\gamma$ release was slightly lower when MR-deficient BMDMs 
were used, whereas TNF $\alpha$ secretion was similar in both genotypes (Figure 3.21 C). IL-17A was hardly detected in the culture medium, therefore we chose another experimental setting to analyze this cytokine. Th17 polarized 2D2 effector T cells were co-cultured with BMDMs from the $M R^{l y s M}$ and $M R^{f l}$ to test whether IL-17 levels were altered after restimulation with $\mathrm{MOG}_{35-55}$. In this setting, IL-17 production induced by MR-deficient BMDMs was comparable to the control situation (Figure 3.21 D).

\subsubsection{The role of $T$ cells in the pathogenesis of EAE in MR ${ }^{\mathrm{IysM}}$ mice}

\subsubsection{MR-deficiency does not impair MOG-specific T cell priming in vivo}

Having tested $T$ cell priming by $\mathrm{MR}^{\mathrm{lysM}}$ macrophages in vitro we took a closer look at the in vivo situation and analyzed the priming of adoptively transferred 2D2 T cells in MR ${ }^{l y s M}$ mice after immunization. In line with the in vitro experiments, no major changes in $T$ cell proliferation were observed in the spleen and the inguinal lymph nodes, although, for unknown reasons, $T$ cells proliferated somewhat faster in the axillary lymph nodes of the mutant mice compared to the $\mathrm{MR}^{\mathrm{fl}}$ littermates (Figure 3.22).

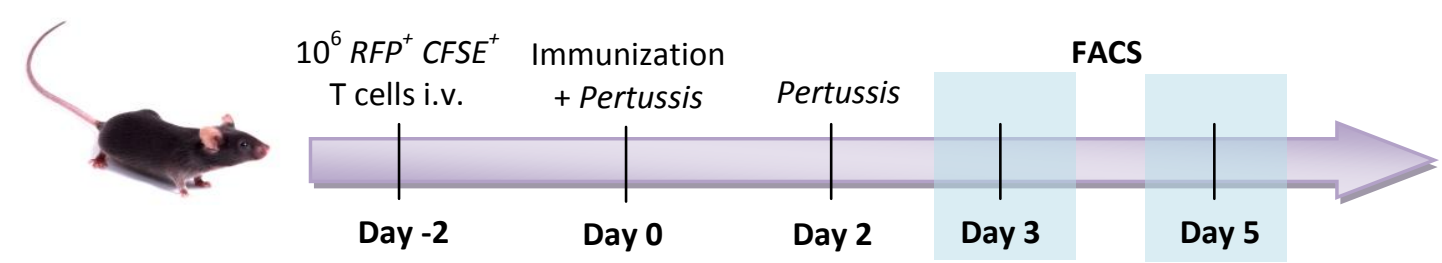

Spleen

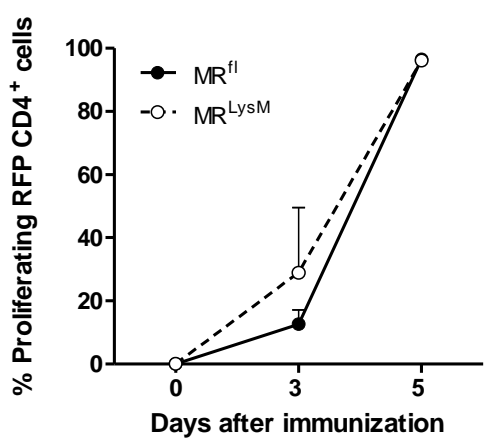

Inguinal LN

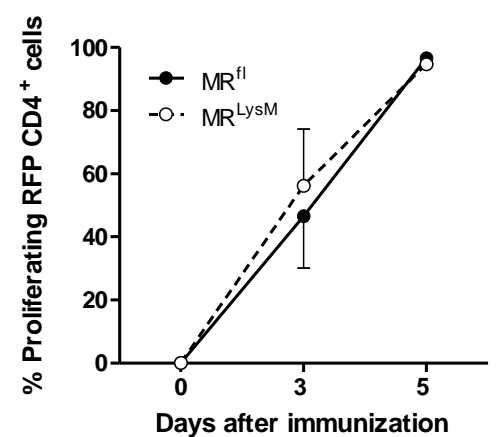

Axillary LN

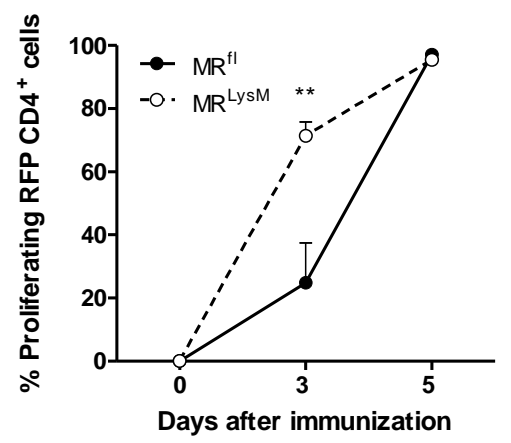


Figure 3.22 | T cell priming in MR ${ }^{\text {lysM }}$ mice in the early phases of EAE is unaffected. MOG-specific T cells were purified from RFP ${ }^{+} 2 \mathrm{D} 2$ mice and subsequently labeled with CFSE. $1 \cdot 10^{6}$ of these T cells were i.v. injected into $M R^{f l}(n=3)$ and $M R^{l y s M}(n=3)$ mice. Two days later $E A E$ was induced by active immunization with $\mathrm{MOG}_{35-55} 3$ and 5 days after immunization, spleens, draining (inguinal) lymph nodes and non-draining (axillary) lymph nodes were analyzed by FACS to determine the proliferation rate of the injected RFP $2 \mathrm{D} 2 \mathrm{~T}$ cells. Values were analyzed using the unpaired Student's t-test and are depicted as mean \pm SEM; **, $\mathrm{p}<0.01$.

\subsubsection{Secretion of Th1 and Th17 cytokines during EAE is impaired in MRlysM mice}

Up to this point it appeared that T cell priming and migration to the CNS were not relevant for the attenuation of EAE in MR ${ }^{l y S M}$ mice. However, MR-deficiency in myeloid cells might alter the phenotype of effector T cells. Therefore, the capacity of antigen-specific peripheral $\mathrm{T}$ cells to produce pro-inflammatory cytokines was studied. Total splenocytes and lymph node cells from immunized mice were isolated briefly before the onset of EAE and restimulated with $\mathrm{MOG}_{35-55}$ in vitro. Subsequently, secretion of Th1 and Th17 cytokines was determined by ELISA as a measure of $\mathrm{T}$ cell effector functions. The results showed that antigen-specific effector $\mathrm{T}$ cells from $\mathrm{MR}^{\mathrm{lysM}}$ did not secrete pro-inflammatory cytokines as efficiently as the ones from $M R^{f l}$ mice. Importantly, secretion was impaired for IL-17A, IFNy, TNF $\alpha$ and GM-CSF, the main pro-inflammatory cytokines involved in the pathogenesis of EAE (Figure 3.23).

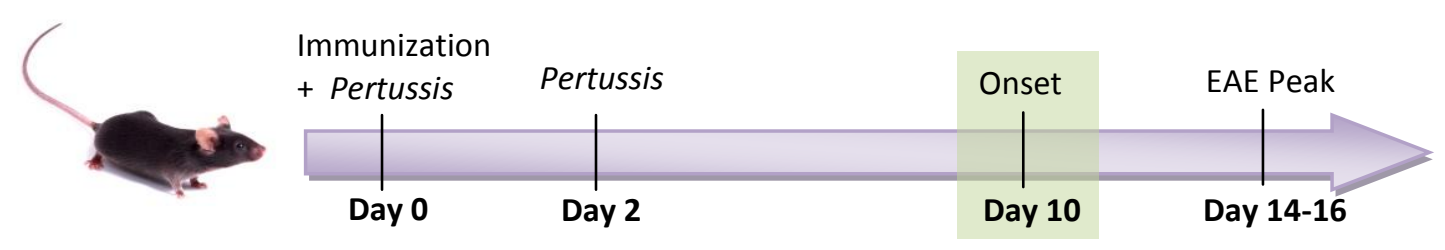


IL-17A

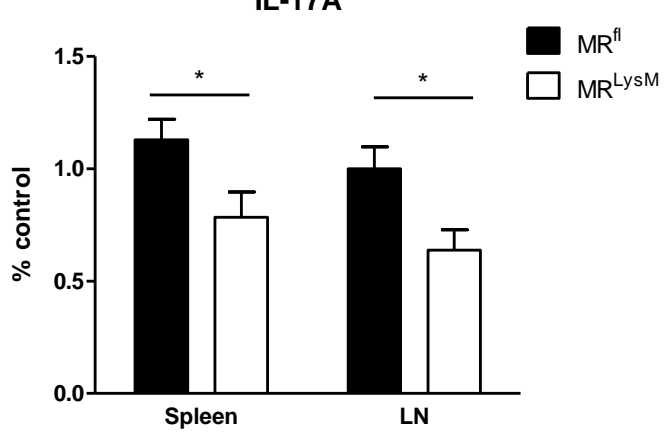

IFN $\gamma$

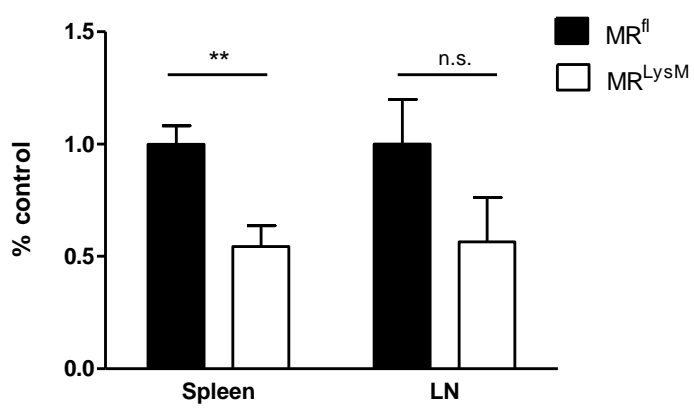

GM-CSF

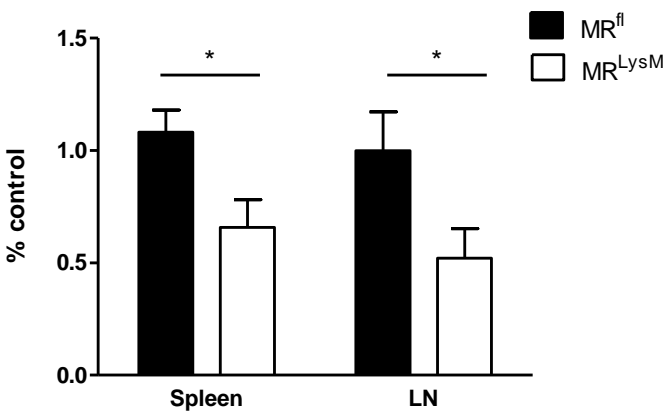

TNF $\alpha$

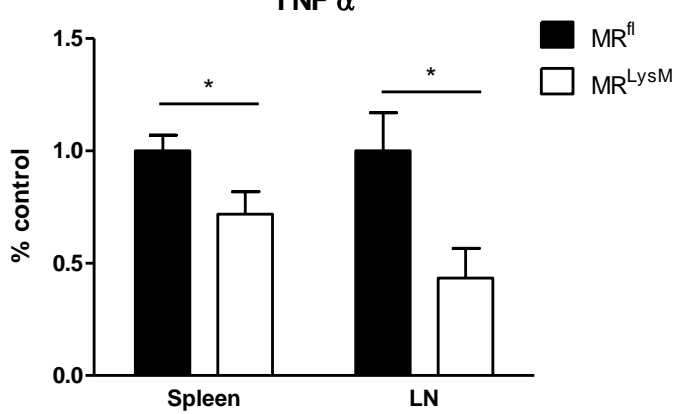

Figure 3.23 | Secretion of pro-inflammatory cytokines by effector $T$ cells is reduced in the secondary lymphoid organs of $M R^{1 y s M}$ mice before the onset of EAE. $M R^{f \mid}$ and $M R^{\mid y s M}$ mice were immunized with $\mathrm{MOG}_{35-55}$ (see 2.3.1) and spleens and lymph nodes were removed the onset of the disease (day 10 after immunization). Single cell suspensions were prepared and $3 \cdot 10^{5}$ lymph node cells or $6 \cdot 10^{5}$ splenocytes were re-stimulated in vitro by adding $20 \mu \mathrm{g} / \mathrm{ml}$ of $\mathrm{MOG}_{35-55}$ for $72 \mathrm{~h}$ (4 independent experiments). The supernatants were analyzed by ELISA to quantify the amounts of IL-17A $(n=8,10)$, GM-CSF $(n=9,9)$, IFNy $(n=10,9)$ and TNF $\alpha(n=8,7)$. The mean value of the $M R^{f l}$ control group in each individual experiment was set to 1 and the values obtained from $\mathrm{MR}^{\mathrm{lys}}$ mice were normalized to the controls. Values were analyzed using the unpaired Student's t test and are depicted as mean \pm SEM; (n.s.), $p \geq 0.05 ;{ }^{*}, p<0.05 ;{ }^{* *}, p<0.01$.

\subsubsection{MR deficiency in myeloid cells leads to increased numbers of peripheral Treg cells}

Treg cells play an important role in the control of autoimmune responses such as EAE (Kohm et al. 2003), although their exact mechanisms have been a matter of debate for many years (Zhang et al. 2004; McGeachy et al. 2005; Tischner et al. 2006; O'Connor et al. 2007; Korn et al. 2007b). Furthermore, a close relationship between M2 macrophages and the increased 
appearance of Treg cells in the context of CNS inflammation has been proposed (Keating et al. 2009; Zhang et al. 2009). Therefore, and in view of the reduced cytokine expression by peripheral encephalitogenic T cells, we decided to analyze the frequency of Treg cells in spleen and lymph nodes briefly before the onset of the disease. In line with the previously observed polarization of macrophages to the M2 phenotype, we also found higher numbers of Treg cells in the spleen and the lymph nodes of $M R^{l y s M}$ mice compared to $M R^{f l}$ controls (Figure 3.24 B). This was true not only for immunized mice, but also for naïve ones, and reached significance in the spleen. In addition, the spinal cord was analyzed at the peak of the disease. However, in this case the frequency of Treg cells among all infiltrating $\mathrm{CD} 4^{+} \mathrm{T}$ cells was similar in $\mathrm{MR}^{\mathrm{fl}}$ and $\mathrm{MR}^{\mathrm{lysM}}$ mice (Figure $3.24 \mathrm{C}$ ).

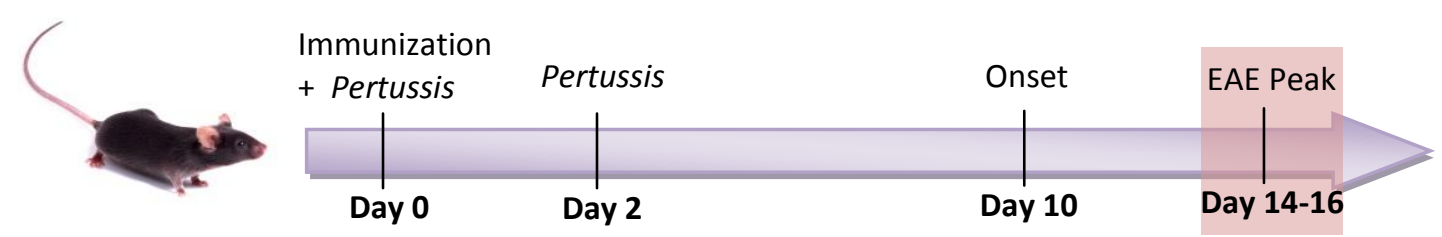

A
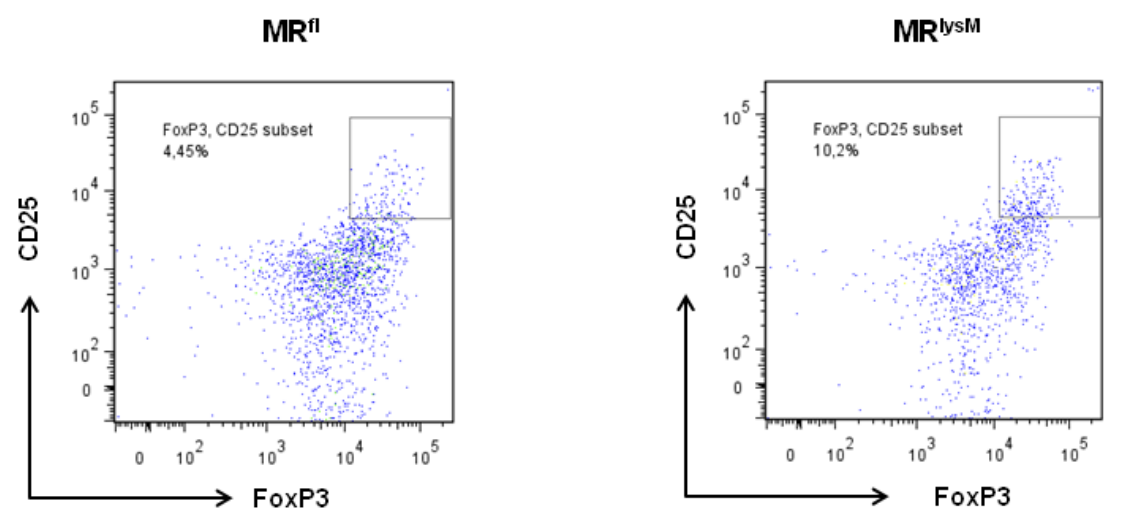

B
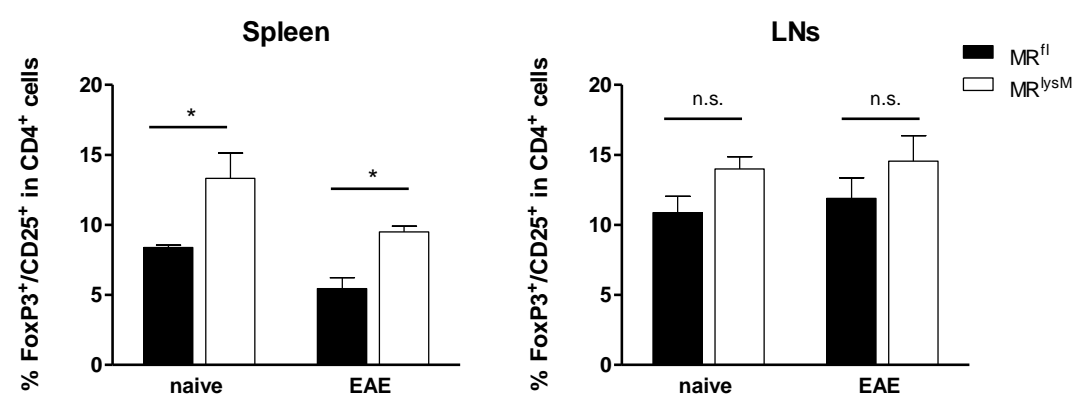

C

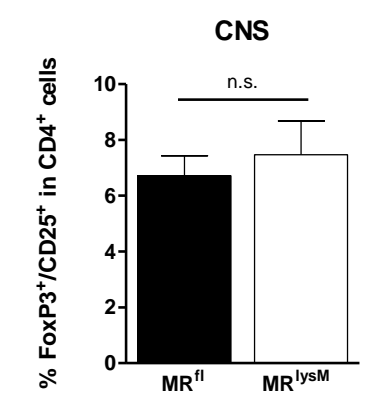


Figure 3.24 | The frequency of Treg cells in peripheral lymphoid organs is increased in MRlysM mice. $(A, B)$ Single-cell suspensions from spleens and lymph nodes of $M R^{f l}$ and $M R^{l y s M}$ mice were obtained either under healthy conditions $(n=4,4)$ or at day 10 after EAE induction $(n=3,3)$. (C) To analyze the percentage of Treg cells in the CNS, spinal cords from $\mathrm{MR}^{\mathrm{fl}}(n=11)$ and $\mathrm{MR}^{\mathrm{IySM}}(\mathrm{n}=12)$ mice at the peak of EAE were homogenized and mononuclear cells were separated via Percoll gradient. The cells were first stained with Abs against CD4 and CD25 and then permeabilized to allow access of the anti-FoxP3 Ab to its target antigen. Total living cells were first gated on the $\mathrm{CD} 4^{+}$cell population, among which the $\mathrm{CD} 25^{+} \mathrm{FoxP}^{+}$cells were defined as Treg cells. Values were analyzed using the unpaired Student's t-test and are depicted as mean \pm SEM; (n.s.), $p \geq$ $0.05 ;{ }^{*}, \mathrm{p}<0.05$.

Taken together, the $M 2$ polarized macrophages in $M R^{\text {lysM }}$ mice appear to foster the generation of Treg cells in peripheral lymphoid organs. These cells act locally rather than migrating to the spinal cord. Furthermore, MR-deficient myeloid cells within the CNS are also not able to induce Treg cells locally.

\subsubsection{The phenotype of microglia during EAE is altered in MR ${ }^{\mathrm{lys} M}$ mice}

The myeloid compartment is composed of both circulating and resident cells, with the latter ones being mostly derived from common myeloid precursors that migrate to the different tissues during the early development. Within the CNS microglia represents the population of myeloid cells that are in charge of responding to inflammatory insults, and of surveillance and homeostasis in the absence of inflammation. Microglial cells share many features with peripheral resident macrophages, including their response to GCs. Several earlier in vitro studies revealed that GCs inhibit microglia proliferation (Ganter et al. 1992), NO production (Jun et al. 1994) and cytokine release (Chao et al. 1992). Moreover, microglia also expresses the GR as well as the MR, and it has been suggested that the two receptors might mediate opposite effects of GCs (Tanaka et al. 1997). It is noteworthy that previous studies had confirmed that LysM $^{\text {Cre }}$ transgenic mice were a suitable tool to disrupt genes in microglial cells (Schweingruber et al. 2011). Therefore, we aimed to investigate whether the deletion of the MR in microglia had any influence on their properties and whether these potential changes might contribute to the ameliorated disease in $\mathrm{MR}^{\mathrm{lys} \mathrm{M}}$ mice. 
Mononuclear cells were eluted from the spinal cord of $M R^{l y s M}$ and $M R^{f l}$ mice at the peak of EAE and analyzed by FACS. The activation state of microglial cells, defined as $\mathrm{CD} 11 \mathrm{~b}^{+} \mathrm{CD} 45.2^{\text {low }}$, can be distinguished by FACS analysis on the basis of Ly6C surface levels. The frequency of reactive microglia, characterized by higher Ly6C surface levels, was reduced in MR-deficient animals as compared to controls (Figure 3.25 B). This indicates that the activation of microglial cells was impaired in the absence of the $M R$, which might contribute to the amelioration of the disease in the mutant mice.

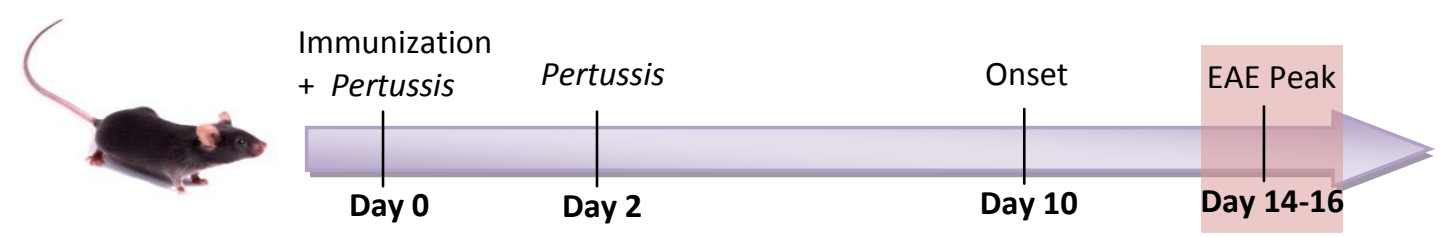

A
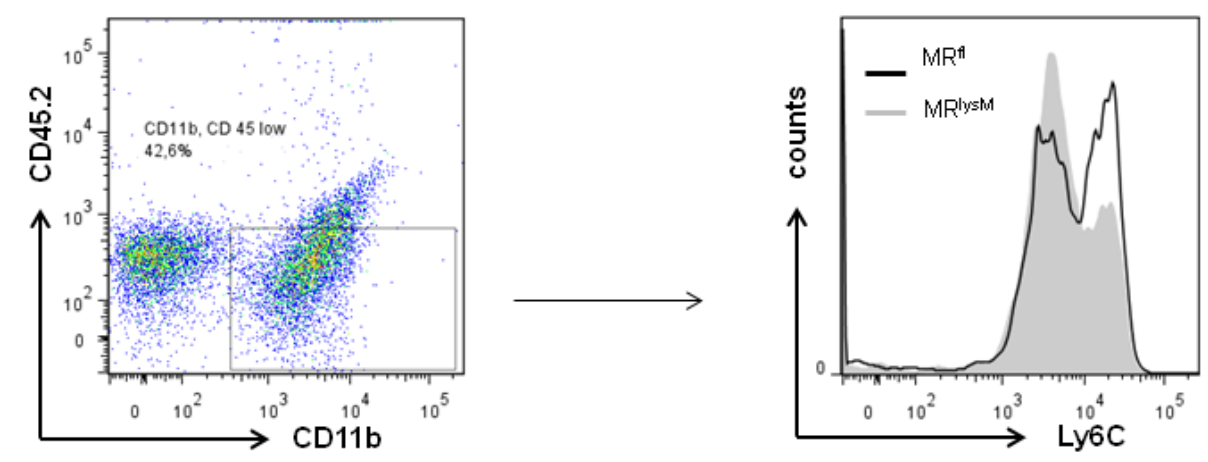

B

Microglia

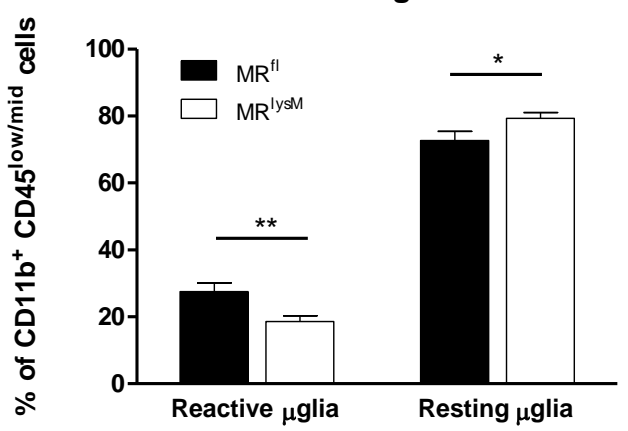


Figure 3.25 | The frequency of reactive microglia in the spinal cord of MR ${ }^{\text {lysM }}$ mice is reduced. Spinal cords from $M^{f l}(n=20)$ and $M R^{l y s M}(n=21)$ mice were removed at the peak of EAE, homogenized and the mononuclear cells were separated using a Percoll gradient. The cells were incubated with Abs against CD11b, CD45.2 and Ly6C and analyzed by FACS. Following the gating strategy published by Prinz et al., microglial cells were distinguished from infiltrating myeloid cells by their lower expression levels of CD45.2, and reactive microglial cells were identified by their high Ly6C expression levels. Values were analyzed using the unpaired Student's t-test and are depicted as mean \pm SEM; *, p <0.05; **, p <0.01. 


\section{DISCUSSION}

\subsection{EAE therapy with betamethasone nanoparticles}

The popularity of nanoparticles for diagnostic and therapeutic applications is gaining ground over the years, and this is reflected by the increasing number of publications dealing with this topic. In MS research, the use of nanoparticles has several advantages compared to conventional methods. Magnetic nanoparticles improve the contrast and the image resolution in MRI studies, and allow the tracking of phagocytic cells in the affected brain areas of rats suffering from EAE (Merodio et al. 2000; Rausch et al. 2004; Mikita et al. 2011; Hunger et al. 2014). Furthermore, the benefits of nanocarriers for the delivery of therapeutic drugs have also been studied. For instance, encapsulation in PEG-liposomes increases the circulation time of the contained drug and promotes accumulation in inflamed target organs. An increased brain bioavailability of the antioxidant tempamine was reported in mice suffering from EAE when they were treated with nanoliposomes (Kizelsztein et al. 2009). Moreover, since phagocytic immune cells engulfing the nanoparticles function as APCs, different nano-carriers have been tested to deliver tolerogenic peptides together with adjuvant molecules to establish T cell tolerance (Büyüktimkin et al. 2012; Yuan et al. 2014; Hunter et al. 2014b). In this project, we exploited the advantages of these delivery systems to improve the properties of GCs. Liposomal formulations of GCs have already been used before in different animal models of autoimmunity with promising results (Metselaar et al. 2003; Schmidt et al. 2003; Linker et al. 2008; Schweingruber et al. 2011). However, this doctoral thesis evaluates for the first time the therapeutic potential of an innovative nanomaterial which combines inorganic carrier molecules with the therapeutic organic compound in its crystallized structure (Heck et al. 2015). The in vitro and in vivo experiments presented here showed that the used IOH-NPs, composed of $\mathrm{ZrO}_{2}$ and $\mathrm{BMZ}$-phosphate, efficiently delivered the GC resulting in the consequent immunomodulation. Moreover, the present study revealed that myeloid cells were specific targets during the treatment of EAE. 


\subsubsection{BNPs are anti-inflammatory in different immune cell types, albeit with different efficacy}

Among the plethora of effects that GCs exert on the immune system (see 1.3.2), induction of T cell apoptosis and alternative polarization of macrophages are well characterized and have been proposed as mechanisms involved in the immunomodulation by GCs (Tuckermann et al. 2005; Varga et al. 2008). Therefore, these two cellular events were analyzed and served as parameters to assess the efficacy of the BNPs. Previous unpublished data of our group indicated that, when used at similar concentrations as Dex in vitro, the effect of BNPs was comparable to the free GC. Both GC formulations led to a similar reduction of proinflammatory molecules in macrophages, and induced T cell apoptosis to the same extent. With the present study I corroborated these results and additionally confirmed that these effects were mediated by the GR, as shown by the abrogation of GC modulation in the absence of the receptor (Figure 3.5). Furthermore, the consequences of nanoparticle administration in vivo were evaluated. $10 \mathrm{mg} / \mathrm{kg}$ of i.p.-injected BNPs decreased the expression of MHC class II and the co-stimulatory molecule CD86 on the surface of macrophages isolated from the peritoneum. Moreover, these macrophages were not able to respond to LPS/INFy stimulation to the same extent as macrophages from mice receiving only vehicle (Figure 3.2). In line with the in vitro experiments, the phenotype of peritoneal macrophages isolated from mice receiving BNPs did not differ from the one of mice treated with free Dex. However, the analysis of T cell apoptosis in vivo revealed differences between both treatments. While Dex efficiently reduced T cell numbers already $24 \mathrm{~h}$ after injection, BNPs barely affected T cell survival $72 \mathrm{~h}$ later (Figure 3.3). This was a first sign of the specificity of the nanoparticles, the efficacy of which appeared to change depending on the cell type in vivo but not in vitro. This is not surprising considering that, due to their size between 40-90 nm, BNPs are expected to be pinocytosed by macrophages and inflammatory monocytes rather than by T cells (Weissleder et al. 2014). Evidence for this is provided by the fact that in cell cultures of splenocytes, lymph node cells or peritoneal lavages, CD $11 \mathrm{~b}^{+}$cells appeared to incorporate higher amounts of BNPs than T cells or B cells (Figure 3.1). In line with this finding, previous data from our group had revealed that the pro-apoptotic effect of the BNPs on T cells in vitro is attenuated at lower BNP concentrations, a tendency that was 
not observed with free GCs. This indicates that at high drug concentrations various immune cells are potentially targeted by the BNPs, but at reduced dosage the different cell types compete for the uptake of the nanoparticles, and in that case myeloid cells are the preferred target of BNPs.

\subsubsection{Myeloid cells are major targets of BNPs in EAE therapy}

Our group and others had already demonstrated that nanoformulations such as liposomes are suitable vehicles to treat autoimmune diseases with GCs (Metselaar et al. 2003; Schmidt et al. 2003; Linker et al. 2008; Schweingruber et al. 2011). Schweingruber and colleagues showed that liposomes loaded with prednisolone $(\mathrm{PL})$ are more potent than free GCs in reducing paralysis symptoms in mice with $\mathrm{EAE}$, even at a reduced dosage and application frequency (Schweingruber et al. 2011). In the present study, BNP i.p. injection on three consecutive days during disease onset had a significant and sustained effect on EAE progression, diminishing the clinical score by at least 3 points in a scale from 1 to 10 (Figure 3.6). Titration of BNPs in subsequent EAE experiments showed that this therapeutic effect was concentration-dependent. Furthermore, when different GCs were applied in parallel, the efficacy of the BNPs was comparable to Dex and BMZ. However, in contrast to PL, BNPs did not appear to have a superior potency. This differential effect between the two nanocompounds is surprising considering that $\mathrm{BMZ}$ is almost six times more potent than prednisolone when tested as a free GC (Van Rensburg 2011). Thus, the mechanisms of action employed by liposomes and $\mathrm{ZrO}_{2}$ nanoparticles might not be the same. Regarding the effect of the inorganic structure of the particles, ENPs neither improved nor worsened the EAE symptoms of the mice, which indicates that BNP therapy is well tolerated, at least in this disease model.

Mirroring MS, demyelination and massive leukocyte infiltration into the spinal cord are major histopathological hallmarks of chronic progressive EAE in C57BL/6 mice (Berard et al. 2010). It is known that GC therapies hamper the entry of immune cells into the CNS via restoration of the BBB (Rosenberg et al. 1996; Pitzalis et al. 2002), re-direction of T cells 
(Fischer et al. 2013) and induction of apoptosis in diverse immune cell subsets (Leussink et al. 2001; Tuckermann et al. 2005). The reduced number of immune infiltrates, in combination with the M2-polarizing effect of GCs on both CNS resident and migrating myeloid cells, helps improving the demyelinating pathology. The present study shows that BNP treatment prevented demyelination to some extent and significantly reduced monocyte/macrophage infiltration into the CNS (Figure 3.7, Figure 3.8). However, T cell numbers within the spinal cord were unaffected. The decrease in T cell infiltrates after Dex treatment has been reported in the past (Schweingruber et al. 2014), hence our histological data indicates once again that BNPs might not act efficiently on T cells when applied in vivo.

It was previously shown in this work that the anti-inflammatory effects of the BNPs required the GR both in vitro and in vivo (Figure 3.4, Figure 3.5). Thus, to elucidate the cellular target of the BNPs in the treatment of EAE, different cell type-specific GR knock-out mice strains were employed. $G R^{l c k}$ mice, devoid of the GR in T cells, responded to the BNP therapy similarly to $\mathrm{GR}^{\mathrm{fl}}$ controls (Figure 3.9 A). In other words, the presence of the GR in T cells was dispensable for the treatment, and therefore this cell type is probably not the major target of the BNPs. The same was the case for $G R^{S L O 1 C 1}$ mice, where the $G R$ is absent in endothelial cells of the BBB (Figure 3.9 D). It is worth to mention that Dex application in those mice led to similar results as BNP treatment (data not shown). This suggests that the well-known protective role of GCs regarding BBB integrity (Paul and Bolton 1995; Engelhardt 2000) might not be essential for their therapeutic effect during $E A E$, but rather an indirect consequence of the therapy (as already postulated in Wüst et al. 2008). The third genotype tested was the $G^{\text {lysM }}$ mouse strain, where the Cre-mediated excision of the GR affects only myeloid cells. In contrast to the other studied genotypes, these mice were completely refractory to the BNP therapy and presented with a disease progression identical to mutant mice receiving only vehicle (Figure 3.9 B). In view of these data, it can be stated that myeloid cells are preferred targets of the BNPs in the treatment of EAE.

Since T cells are known to be major mediators of conventional GCs during EAE (Wüst et al. 2008; Fischer et al. 2013; Schweingruber et al. 2014), we can also conclude from our experiments that BNPs employ a different route of action than free GCs. This change in cell 
specificity had already been observed by Schweingruber and colleagues using PL, the liposomal formulation of GCs (Schweingruber et al. 2011). However, PL treatment in GR ${ }^{\text {lysM }}$ mice led to a mild therapeutic effect, meaning that, in contrast to BNPs, PL had a residual effect in other cell types besides myeloid cells. Considering the undesirable consequences of GCs' off-target effects, the tighter specificity of BNPs might represent an advantage of $1 \mathrm{OH}-$ NPs over liposomes.

\subsubsection{Are BNPs a potential solution to GC-derived side effects?}

Short-time high-dose GCs are a mainstay for the treatment of acute relapses of MS, but whether a sustained GC treatment would decrease the relapse rate or prevent the worsening of brain lesions remains controversial (Zivadinov et al. 2001; Then Bergh et al. 2006; Sorensen et al. 2009; Ravnborg et al. 2010; Ciriaco et al. 2013). The promising therapeutic potential of this approach claims for more clinical studies, but the broad spectrum of GC-associated side effects is an important limiting factor for the use of prolonged high dose pulsed GCs (Moghadam-Kia and Werth 2010; Weinstein 2012; Ciriaco et al. 2013; Hunter et al. 2014; Hwang and Weiss 2014). Responsible for these side effects is the fact that the GR is ubiquitously expressed (Rhen and Cidlowski 2005), and therefore GCs modulate not just immune responses, but also salt-water homeostasis, glucose metabolism, bone re-adsorption and mood variations, among others. In order to circumvent these consequences of GCs several strategies have been tested, for instance dissociating ligands that act only via the trans-repressing mechanism of the GR (Wüst et al. 2009). However, none of these compounds could be translated into a successful therapy with clinical use so far. In this doctoral thesis we propose the targeting of GC therapy to the myeloid cell compartment as a means to solve this issue.

As a first approach, the occurrence of side effects was analyzed in the context of the experimental protocol used for the EAE experiments, consisting in short-time daily i.p. injection of a moderate dose of GCs (10 mg/kg). A small increase in fasting glucose levels was observed in both Dex- and BNP-treated animals compared to their respective controls, 
although this increase was not significant (Figure 3.11 A). Furthermore, a marked induction of hepatic enzymes involved in gluconeogenesis was observed shortly after Dex or BNP injection (Figure $3.11 \mathrm{C}$ ), indicating that both Dex and BNPs act similarly on hepatocytes increasing the production of glucose. However, it could also be expected that Kupfer cells, the phagocytic liver-resident macrophages, incorporated a considerable proportion of the injected nanoparticles. Then, hypothetically, BMZ loaded in the BNPs might polarize Kupfer cells to a M2-like state, reported to prevent insulin resistance (Odegaard et al. 2008; Huang et al. 2010), thereby counteracting the elevated blood glucose levels. This and other possible compensatory mechanism should be evaluated in the future with different experimental conditions. Similarly to the results observed regarding glucose levels, no considerable changes in stomach weight occurred (Figure 3.13). For the analysis of GC-derived osteoporosis, serum levels of the hormone osteocalcin were measured. Osteocalcin is a promising cognate marker, since it is strongly reduced shortly after GC application and has been shown to be a link between GC-induced bone remodeling and energy metabolism (Brennan-Speranza and Conigrave 2015). However, no differences in osteocalcin were found in the serum of mice treated with Dex or BNPs (Figure 3.14). The last side effect analyzed was muscle wasting. We found that mice receiving Dex presented with a slightly reduced muscle weight, whereas in the BNP-treated ones the difference was hardly perceptible (Figure 3.12). In any case, none of those values were statistically significant, and probably a longer duration of the GC treatment will be needed to identify changes in muscle mass.

Collectively, the analysis of short-term BNPs side effects did not provide clear information, since not even the free GC induced significant changes in the studied parameters. Published data from our group and others showed strong metabolic effects derived from prolonged Dex treatment (Waddell et al. 2008; Reichardt et al. 2014). Therefore further experiments will be performed with longer GC exposure periods or higher concentrations. Only applying this alternative protocol we will be able to determine whether BNPs are better tolerated than conventional GC therapies. 


\subsubsection{BNP therapy: open questions and perspectives}

The data presented here show that BNPs, in spite of modulating both macrophage phenotype and T cell responses in vitro, specifically target the myeloid compartment in vivo. This targeted delivery of GCs opens the path to new therapeutic options. One alternative that was tested in this thesis was the combination of free GCs and BNPs in order to obtain an additive effect of both $\mathrm{T}$ cell suppression and macrophage M2 polarization. Indeed, the administration of suboptimal doses of Dex and BNPs together showed a superior potency in the treatment of EAE than the same amounts of the individual drugs alone (Figure 3.10). Hence, combined therapy with BNPs and free GCs might increase the therapeutic efficacy of the drug allowing the use of reduced dosage, with the consequent advantages regarding side effects and patient compliance.

An important factor for the therapeutic success of the nanoparticles is their biodistribution. Generally, particles with a size of $10-100 \mathrm{~nm}$ tend to accumulate in organs where macrophages are present, like spleen, liver, lymph nodes and bone marrow (Weissleder et al. 2014). Moreover, the delivery route via inflammatory monocytes should lead to higher concentrations of the drug at the site of inflammation, meaning that the use of BNPs may achieve not only cell type-specificity, but also direct targeting of the damaged tissue. Evidence of this phenomenon was provided by Metselaar and colleagues, who showed that rats receiving $\mathrm{PL}$ recovered from adjuvant-induced experimental arthritis (AIA) thanks to delivery of the GC in the inflamed joint (Metselaar et al. 2003). Also Merodio and colleagues observed CNS localization of albumin nanoparticles in a rat model of EAE, and suggested that macrophages and microglia were involved in this distribution (Merodio et al. 2000). Unfortunately, we were not able to determine whether BNPs specifically targeted the injured spinal cord in the treatment of EAE. The presence of the fluorescent molecule FMN in the BNPs allowed their identification by FACS in different cell subsets in culture, however the attempts to track the particles in different organs after i.p. injection were unsuccessful. Noteworthy, in vivo analyses by intra-vital 2-PM after either i.v. or stereotactic injection could not trace the BNPs, meaning that at tolerable doses for the mice the FMN signal of the nanoparticles does not reach the limit of detection. Since $\mathrm{ZrO}_{2}$ has already been coupled to other fluorescent compounds for cell imaging in the past (Heck et al. 2015), the 
biodistribution of the BNPs could be elucidated by means of those alternative nanoparticles in the future.

Questions regarding the BNPs' pharmacokinetics and pharmacodynamics are also important for the success of the therapy. Until now it is unknown whether BNPs bind to albumin or other proteins in the blood, or how long they remain in the circulation before they are cleared from the organism. Something else to take into account is whether the nanoparticles are leaky and release the drug before reaching their target. In the present study, the inefficacy of the BNP treatment in the $G R^{l y s M}$ knock-out mice indicates that, if there was some hypothetical circulating free BMZ, the amount was so low that no therapeutic effect derived from it. With regard to their behavior intracellularly, it is known that medium size nanoparticles, like BNPs, are taken up via micro-/macro-pinocytosis (Weissleder et al. 2014). In theory, the drug would become active in the cytoplasm by enzymatic or spontaneous hydrolysis. However, how the remaining $\mathrm{ZrO}_{2}$ is metabolized and/or excreted is still unknown, and potential toxic effects have to be evaluated. According to the results presented here, the apparent promotion of $\mathrm{CD}^{+} \mathrm{T}$ cell proliferation induced by ENPs in splenocytes (Figure 3.3) suggests that the nanoparticles might not be completely innocuous. Several publications report that $\mathrm{ZrO}_{2}$ particles are hemocompatible and well tolerated by HEK-293 cells and the macrophage cell line RAW-264.7. Moreover, they present low genotoxicity in vitro and after oral administration in larvae of Drosophila melanogaster (Demir et al. 2013; Karunakaran et al. 2013; Saxena et al. 2013). Nonetheless, other studies described a certain immunogenic potential, and showed that $\mathrm{ZrO}_{2}$ may increase the expression of TNF $\alpha$, IL- 6 and IL-1 $\beta$ in macrophages (Obando-Pereda et al. 2014). The in vitro experiments on BMDMs and peritoneal macrophages included in this thesis are discrepant in that regard. However, previous data showed that in some cases IL- 6 and IL-1 $\beta$ expression were slightly increased after ENP exposure in BMDMs (Ring, unpublished data). Hence, it is conceivable that the observed net anti-inflammatory potential of BNPs represents in fact a combination of an even stronger anti-inflammatory effect of BMZ and a mild proinflammatory effect of $\mathrm{ZrO}_{2}$. If this premise was true, it would also explain why BNPs are not as potent as PL in the treatment of EAE. After all, ENPs did not appear to affect EAE severity, which speaks in favor of the general biocompatibility of BNPs at therapeutic concentrations. 
Actually, current studies with BNPs and other variants of IOH-NPs are showing promising results for the analysis and therapy of different autoimmune disorders, such as allergic asthma and acute Graft-versus-Host Disease (Markus et al. 2015; our unpublished data). We therefore believe that this delivery system has the potential to be a successful and better tolerated vehicle for drugs in the future.

\subsection{The role of the MR in myeloid cells during EAE}

The here presented results showing the attenuation of EAE by BNPs provide additional evidence that the different macrophage phenotypes play a crucial role in EAE and MS. But beyond treatment with synthetic GCs, there are other ways to bias macrophage polarization into a deactivating state. A distinctive feature of myeloid cells is that they co-express the GR and the MR, but not $11 \beta-H S D ~ I I$, a situation that allows GCs to access both nuclear receptors (Funder 1997). Interestingly, despite the structural similarities between the GR and the MR (Funder 1997), their activation by GCs has been shown to induce opposing polarization states on macrophages and microglia (Usher et al. 2010; Chantong et al. 2012). This is particularly important for the response to endogenous GCs in mice. Unlike cortisol (the human endogenous $\mathrm{GC}$ ), corticosterone binds to the MR with 10-fold higher affinity than to the GR. In vitro studies showed that, due to this property, low concentrations of corticosterone had immunostimulatory effects via MR signaling, whereas increased amounts of this GC strongly reduced pro-inflammatory genes acting via the GR (Tanaka et al. 1997; Lim et al. 2007; Chantong et al. 2012). These observations suggest that at physiological GC concentrations a great part of the response of myeloid cells to GCs is mediated by the MR. Therefore, we wondered whether the absence of the MR would potentiate the antiinflammatory effects of endogenous GCs via the GR. In the second part of this project the phenotype of mice with MR-deficient myeloid cells was studied in the context of CNS autoimmunity using a cell type-specific knock-out mouse strain, the $\mathrm{MR}^{\mathrm{lysM}}$ mice. 


\subsubsection{MR deficiency in myeloid cells promotes M2-like polarization and causes a milder EAE phenotype}

It is well known that classically activated macrophages contribute to EAE pathology by presenting myelin antigens to T cells (Almolda et al. 2011), secreting pro-inflammatory cytokines (Hendriks et al. 2004) and exerting direct neurotoxicity (Reynolds et al. 2007; Shijie et al. 2009). Other groups have also reported that an imbalance of the $M 1 / M 2$ equilibrium has important consequences for EAE disease progression (Mikita et al. 2011). Therefore, we hypothesized that, since the MR promotes M1 polarization, the deletion of the MR from myeloid cells might alleviate clinical symptoms in mice with EAE. Indeed, when MR ${ }^{\text {lysM }}$ mice were immunized with $\mathrm{MOG}_{35-55}$ they developed a milder form of the disease compared to their littermate controls (Figure 3.15). In a rat model of relapsing EAE, Mikita and colleagues found that M2 macrophages accumulated in the CNS before relapse resolution, and that administration of ex vivo polarized M2 monocytes suppressed EAE symptoms (Mikita et al. 2011). Schweingruber and Li had previously shown that, at the peak of EAE, mononuclear cells isolated from the spinal cord of $M R^{\text {lysM }}$ mice express higher levels of typical M2markers, mainly Arg-1, whereas the expression of the M1-gene iNOS is markedly downregulated (Li 2013). In these mice, the constitutively increased proportion of M2-polarized macrophages, which results from the exclusive GR binding of endogenous corticosterone, appears to stop disease progression earlier than in wt mice, although it is not enough to completely abrogate EAE.

In line with the ameliorated EAE clinical course, the histopathological characteristics of $M R^{\text {lysM }}$ mice also differed from the ones of $M R^{f l}$ controls. Similarly to the above described effects of BNP treatment, spinal cord infiltration by $\mathrm{CD}^{+} \mathrm{T}$ cells was comparable in both groups, whereas $\mathrm{MAC3}^{+}$infiltrates were significantly reduced in the knock-out mice (Figure 3.16). It is worth to mention that the nature of those $\mathrm{MAC}^{+}$cells is hard to determine on the histological basis, since this surface marker is shared by infiltrating monocytes/macrophages, PVMs and activated microglia (Giulian and Baker 1986). The FACS analysis of spinal cord mononuclear cells corroborates that, at the peak of EAE, the proportion of reactive microglia is indeed reduced in $\mathrm{MR}^{\mathrm{lysM}}$ mice (Figure 3.25). 
Furthermore, the percentage of inflammatory monocyte/macrophages infiltrating the spinal cord was reduced as well (Figure 3.18). However, with the current data we cannot definitively conclude that there was less macrophage infiltration into the spinal cord. It can only be stated that those myeloid cells within the CNS, which are pro-inflammatory in nature, were less frequent. In accordance with this phenotype, a significantly lower percentage of demyelination and partially preserved axonal density were observed (Figure 3.17). Previous data from Li showing decreased NO release and increased phagocytic capacity in MR ${ }^{\text {lysM }}$ BMDMs support these results (Li 2013). NO has neurotoxic effects (AboulEnein et al. 2006; Zindler and Zipp 2010) and M2 macrophages and microglia are known to promote neuronal regeneration via clearance of myelin debris (Neumann et al. 2009). Therefore, a direct connection between the clinical course of EAE and the altered phenotype in the MR-deficient monocyte/macrophages can be drawn. Moreover, we suspect that the reduced proportion of reactive microglia also contributes to the observed phenotype. Microglia, since they originate from myeloid precursors in the yolk sac, have also been reported to be a target of the LysM $^{\text {Cre }}$ mediated gene deletion in mice (Prinz et al. 2008; Schweingruber et al. 2011). It has been shown that blockade of the MR with the antagonist spironolactone counteracts pro-inflammatory effects induced by aldosterone or corticosterone in a mouse microglia cell line, which confirms a role of the MR in microglia activation as well (Chantong et al. 2012). However, it cannot be assured yet whether the reduction in reactive microglia was due to the MR deficiency or just a secondary effect of the anti-inflammatory CNS milieu. To shed light on this question, in vitro experiments with primary microglia cells from $\mathrm{MR}^{\mathrm{lysM}}$ mice should be performed.

\subsubsection{MR deletion in myeloid cells alters T cell responses during EAE}

The altered polarization state of MR-deficient myeloid cells could partially explain the improved clinical disease course in the $\mathrm{MR}^{\mathrm{lysM}}$ mice, but it was not clear yet whether other cell types contributed to this phenotype. Although the deletion of the $M R$ in $M R^{\text {lysM }}$ mice is restricted to the myeloid compartment, myeloid cells act in close collaboration with T cells at different stages of EAE progression. It is actually known that T cells are the most important 
players in the pathomechanisms leading to $E A E$, therefore potential changes in $T$ cell responses were also investigated.

Some myeloid cells, including macrophages, act as professional APCs, and efficient presentation of myelin epitopes is crucial for EAE. This process is involved in the initial priming of T cells, as well as in the maintenance of the CNS inflammatory response in the chronic phase of the disease. As it was previously shown in this thesis, GCs down-regulate MHC class II and co-stimulatory molecules acting via the GR. Therefore we wondered whether antigen presentation was impaired in MR-deficient macrophages. Co-culture of BMDMs with naïve $2 \mathrm{D} 2 \mathrm{~T}$ cells in the presence of $\mathrm{MOG}_{35-55}$ demonstrated that $\mathrm{MR}^{\mathrm{lys} M}$ BMDMs were able to present antigens and induced $T$ cell proliferation as efficiently as $\mathrm{MR}^{\mathrm{fl}}$ cells (Figure 3.21). Next, T cell priming was studied in vivo by adoptive transfer of CFSElabeled 2D2 T cells in $\mathrm{MOG}_{35-55}$ immunized mice. Three days after immunization part of the adoptively transferred 2D2 T cells were already proliferating in the spleen and the lymph nodes (Figure 3.22) However, surprisingly, there was a tendency towards increased T cell proliferation in the $M R^{l y s M}$ mice, reaching significance in the axillary lymph nodes. Since the in vitro experiments on $\mathrm{BMDMs}$ indicated that $\mathrm{T}$ cell activation by $\mathrm{MR}^{\mathrm{lysM}}$ macrophages was neither impaired nor increased, it could be hypothesized that other cell types rather than macrophages are responsible for the apparently enhanced T cell proliferation in vivo. DCs are the major cell type involved in T cell priming during EAE (Greter et al. 2005). However, it is not known whether they express significant levels of $M R$ and furthermore, Lys $^{\text {Cre }}$ transgenic mice do not appear to target any type of DC with high efficiency (Prinz et al. 2008). Therefore, the question why the proliferation rate in the axillary lymph nodes was increased remains to be answered. In any case, the presumed differences in T cell priming did not lead to changes in $T$ cell infiltration between $M R^{l y s M}$ and control mice. Hence we believe that this effect was of minor importance for the observed EAE alleviation.

During $E A E$, macrophages have also the task of shaping $T$ cells responses by providing a specific cytokine environment. This study showed that in vitro BMDM activation induced the release of inflammatory cytokines such as IL- 6 and TNF $\alpha$, however this induction was milder in BMDMs from $M R^{\text {lysM }}$ mice. On the contrary, IL-10 secretion by $M^{l y s M} B M D M s$ was 
increased compared to the controls (Figure 3.20). Thus, we wondered whether the altered cytokine profile in macrophages would lead to changes in the T cell activation pattern. Coculture of $2 \mathrm{D} 2$ T cells with $\mathrm{MR}^{\mathrm{lysM}}$ BMDMs led to a reduced secretion of IFNy by $\mathrm{T}$ cells, whereas TNFa release, in contrast, was unaffected (Figure 3.21). Levels of other T cell cytokines such as IL-4, IL-17, IL-2 and IL-10 were barely detectable. These data suggest that, although $\mathrm{T}$ cell priming and T cell migration appeared to be unaltered, the activated state of the primed T cells might be milder once they reach the CNS. Previous data from Li, however, refute this hypothesis. In his experiments the expression levels of IL-17A and IFNy in the spinal cord of $M R^{l y s M}$ mice was comparable to the one in $M R^{f l}$ mice (Li 2013). In the present work, we did find changes in the functionality of T cells during EAE, but they were restricted to the periphery. Ex vivo re-stimulation of splenocytes and lymph node cells revealed that $T$ cells from the knock-out mice secreted less pro-inflammatory cytokines than the controls shortly before disease onset (Figure 3.23). IFNy, TNF $\alpha$ and IL-17A levels were significantly reduced, indicating that Th1 and Th17 responses might be impaired in the $M R^{\text {lysM }}$ mice. Importantly, GM-CSF, the only T cell cytokine known to be essential for EAE development (Codarri et al. 2011; El-Behi et al. 2011), was also significantly lower in both the spleen and the lymph nodes of $M^{l y s M}$ mice. One of the roles of GM-CSF is promoting the release of myeloid progenitors from the bone marrow, therefore the reduction of this cytokine might account for the lower percentages of circulating inflammatory monocytes found in MR $\mathrm{R}^{\mathrm{lys} \mathrm{M}}$ mice (Figure 3.18), and might explain the presumably reduced monocyte infiltration into the spinal cord.

Having demonstrated that peripheral $T$ cells of $M R^{l y s M}$ mice produced lower amounts of proinflammatory cytokines during EAE, we analyzed whether MR-deficient myeloid cells were directly inducing this phenotype on T cells. In vitro re-stimulation of MOG-specific Th17 cells co-cultured with $M R^{\text {lysM }}$ BMDMs did not affect the protein levels of IL-17A in the culture medium (Figure 3.21). Furthermore, no changes in the proportion of inflammatory monocytes or granulocyte numbers were observed in the spleens and lymph nodes of the $M R^{\text {lysM }}$ mice (Figure 3.19). Altogether, these data exclude a direct connection between the knock-out myeloid cells and the altered T cell functionality in the periphery. One of the major mechanisms of $\mathrm{T}$ cell suppression is mediated by Treg cells. Hence, this cell 
compartment was additionally analyzed. An elevated percentage of Treg cells was found in the spleen and lymph nodes of $M O O_{35-55}$-immunized $M R^{l y s M}$ mice shortly before $E A E$ onset (Figure 3.24). Interestingly, this increased Treg cell population was also found in healthy mice before immunization. Furthermore, it appeared that these peripheral Treg cells did not migrate to the CNS, indicating that their suppressive function might not be antigen-specific. Evidence of this is the comparable proportion of Treg cells in the spinal cords of $\mathrm{MR}^{\mathrm{fl}}$ and $M R^{\text {lysM }}$ mice, in line with the unaltered $T$ cell infiltration and cytokine secretion observed in the CNS. All in all, these results point to Treg cells as secondary mediators of the milder EAE phenotype in the $M R^{\mathrm{lysM}}$ mice.

\subsubsection{MR deletion in myeloid cells partially restores peripheral tolerance}

Summing-up, the presented data suggest that the improved EAE clinical symptoms observed in the $M R^{l y s M}$ mice are the result of two distinct regulatory mechanisms that, moreover, are separately compartmentalized. On the one hand, myeloid-targeted deletion of the MR promotes a deactivating state in microglia and monocytes/macrophages infiltrating the spinal cord. These M2 polarized myeloid cells establish a milder pro-inflammatory milieu within the CNS that prevents tissue damage and supports myelin regeneration. On the other hand, Treg cells act together with M2-monocytes in the periphery restraining effector $\mathrm{T}$ cell responses in secondary lymphoid organs, which also contribute to the overall reduced inflammatory response.

This synchronized intervention of M2-like macrophages and Treg cells has been extensively reported in different contexts. Hu and colleagues showed that adoptive transfer of $\mathrm{CD} 4^{+}$ $\mathrm{CD}^{2} 5^{+}$Treg cells reduced the pro-inflammatory state of peritoneal macrophages, downregulating $\mathrm{MHC}$ class II molecules and CD86 expression, increasing phagocytosis and IL-10 expression and altering the Arg1/iNOS balance (Hu et al. 2012). The macrophage phenotype described by $\mathrm{Hu}$ and colleagues, noteworthy, strongly resembles to the one induced by GCs. Conversely, human anti-inflammatory monocytes have been also shown to induce $\mathrm{CD} 25^{+}$ FoxP3 $^{+}$Treg cells with a potent suppressive activity (Savage et al. 2008). The same authors 
proposed the production of ROS by macrophages as the link between these monocytes and the increased Treg induction (Kraaij et al. 2010). In agreement with the results of this thesis, GC-induced M2-like macrophages have also been reported to promote the generation of Treg cells. In a rat model of experimental autoimmune neuritis, Zhang and colleagues showed that Compound A, a GR agonist that just acts via the trans-repression mechanism, induced M2-like macrophages in vivo and in vitro. Furthermore, treatment with Compound $A$ reduced disease severity and increased the numbers of Treg cells in the lymph nodes, where Th1 and Th2 cytokines were strongly suppressed (Zhang et al. 2009). Another study closely related to ours demonstrated that repetitive stimulation of naive splenic $T$ cells with monocytes that had been matured in the presence of Dex yielded higher proportions of FoxP3 $^{+}$Treg cells in vitro (Varga et al. 2014). Moreover, when these GC-induced monocytes were injected in mice with severe colitis the clinical symptoms rapidly improved, and clusters of FoxP ${ }^{+}$cells were found in the damaged colon. In line with Varga and colleagues, the $M R^{\text {lysM }}$ model, via deletion of the MR from myeloid cells, achieves a sustained M2-like polarization throughout the lifetime of the mouse, which would be equivalent to the repetitive stimulation of naïve T cells to increase the proportion of Treg cells. Unfortunately, Varga and colleagues could not identify the mechanism used by the GC-induced monocytes to induced Treg differentiation, and neither could we yet.

In a way, this effect could be interpreted as a reinforcement of the peripheral tolerance throughout lifetime. As example supporting this notion, a recent report proposed that during pregnancy, fetal tolerance was partly induced by generation of M2-macrophages and Treg cells via soluble factor secreted by the placenta (Svensson-Arvelund et al. 2015). This study highlights the importance of this combined regulatory mechanism, linking innate and adaptive immunity, to prevent potentially harmful immune responses, such as MS and other autoimmune disorders. The data presented in this thesis, moreover, provides evidences of the participation of GCs in the establishment of tolerance via myeloid cells, and opens new paths for alternative therapeutic strategies based on this principle. 


\subsection{Conclusions}

For many years T cells have been considered the major therapeutic targets of GC treatment in neuroinflammatory diseases. However, the experimental data presented in this doctoral thesis show that when GC modulation is restricted to myeloid cells it is also able to change the course of the model disease EAE. This supports the already existing literature that postulate an important role of monocytes/macrophages in the therapy of autoimmune disorders with GCs (Metselaar et al. 2003; Mikita et al. 2011; Schweingruber et al. 2011; Varga et al. 2014). In view of the successful therapeutic effect of the myeloid-targeted therapy and the tight cell specificity of the system, BNPs were proposed as an alternative to overcome GC-associated side effects, but the obtained data did not provide conclusive information in this respect yet.

Furthermore, this study contributes to the understanding of the distinct participation of the GR and the MR in CNS autoimmunity. GR deletion from myeloid cells aggravated the clinical symptoms of EAE and rendered the mice resistant to the BNP therapy, whereas MR ablation in $M R^{\text {lysM }}$ mice had a beneficial effect in terms of disease severity. Additional unpublished data from our group indicates that systemic application of an MR antagonist also ameliorates EAE disease progression, although not to the same extent as the myeloidspecific knock-out. In our view, combining both experimental approaches, namely the delivery of the MR antagonist via nanoparticles, might increase the effective concentration of the drug in myeloid cells improving its efficacy. This and other potential variants of the strategies described in this thesis should be further investigated in the future.

Last but not least, we identified an involvement of M2-polarized monocytes/macrophages, and indirectly of endogenous GCs, in the establishment of peripheral tolerance. Under this new perspective, the above described low-dose pulsed GC protocols gain relevance. Sustained exposure of myeloid cells to GCs, either by synthetic GC application or targeted blockade of the MR, might assist the peripheral regulatory mechanisms in charge of maintaining tolerance; this could lead to a reduced relapse rate or improved recovery in MS patients, as it did in the mouse model of chronic-progressive EAE employed for this study. 


\section{References}

Aboul-Enein F, Weiser P, Höftberger R, Lassmann H, Bradl M (2006): Transient axonal injury in the absence of demyelination: a correlate of clinical disease in acute experimental autoimmune encephalomyelitis. Acta Neuropathol (Berl) 111, 539-547

Abromson-Leeman S, Bronson RT, Dorf ME (2009): Encephalitogenic T cells that stably express both T-bet and ROR gamma t consistently produce IFNgamma but have a spectrum of IL-17 profiles. J Neuroimmunol $\underline{215}, 10-24$

Adorini L, Guéry JC, Trembleau S (1996): Manipulation of the Th1/Th2 cell balance: an approach to treat human autoimmune diseases? Autoimmunity $\underline{23}, 53-68$

Ajami B, Bennett JL, Krieger C, McNagny KM, Rossi FMV (2011): Infiltrating monocytes trigger EAE progression, but do not contribute to the resident microglia pool. Nat Neurosci $\underline{14}, 1142$ 1149

Alberts DS, Muggia FM, Carmichael J, Winer EP, Jahanzeb M, Venook AP, Skubitz KM, Rivera E, Sparano JA, DiBella NJ, et al. (2004): Efficacy and safety of liposomal anthracyclines in phase I/II clinical trials. Semin Oncol $\underline{31}, 53-90$

Almawi WY, Beyhum HN, Rahme AA, Rieder MJ (1996): Regulation of cytokine and cytokine receptor expression by glucocorticoids. J Leukoc Biol $\underline{60}, 563-572$

Almolda B, Gonzalez B, Castellano B (2011): Antigen presentation in EAE: role of microglia, macrophages and dendritic cells. Front Biosci Landmark Ed $\underline{16}$, 1157-1171

Ando DG, Clayton J, Kono D, Urban JL, Sercarz EE (1989): Encephalitogenic T cells in the B10.PL model of experimental allergic encephalomyelitis (EAE) are of the Th-1 lymphokine subtype. Cell Immunol $\underline{124}, 132-143$

Babbe H, Roers A, Waisman A, Lassmann H, Goebels N, Hohlfeld R, Friese M, Schröder R, Deckert M, Schmidt S, et al. (2000): Clonal expansions of CD8(+) T cells dominate the T cell infiltrate in active multiple sclerosis lesions as shown by micromanipulation and single cell polymerase chain reaction. J Exp Med 192, 393-404

Barker CF, Billingham RE (1972): Analysis of local anatomic factors that influence the survival times of pure epidermal and full-thickness skin homografts in guinea pigs. Ann Surg $\underline{176}, 597-604$

Barnett MH, Prineas JW (2004): Relapsing and remitting multiple sclerosis: pathology of the newly forming lesion. Ann Neurol $\underline{55}$, 458-468

Bartholome B, Spies CM, Gaber T, Schuchmann S, Berki T, Kunkel D, Bienert M, Radbruch A, Burmester G-R, Lauster R, et al. (2004): Membrane glucocorticoid receptors (mGCR) are expressed in normal human peripheral blood mononuclear cells and up-regulated after in vitro stimulation and in patients with rheumatoid arthritis. FASEB J Off Publ Fed Am Soc Exp Biol $\underline{18}, 70-80$ 
Batchelor PE, Liberatore GT, Wong JY, Porritt MJ, Frerichs F, Donnan GA, Howells DW (1999): Activated macrophages and microglia induce dopaminergic sprouting in the injured striatum and express brain-derived neurotrophic factor and glial cell line-derived neurotrophic factor. J Neurosci Off J Soc Neurosci 19, 1708-1716

Batoulis H, Recks MS, Addicks K, Kuerten S (2011): Experimental autoimmune encephalomyelitis achievements and prospective advances. APMIS $\underline{119}$, 819-830

Baumann S, Dostert A, Novac N, Bauer A, Schmid W, Fas SC, Krueger A, Heinzel T, Kirchhoff S, Schütz G, Krammer PH (2005): Glucocorticoids inhibit activation-induced cell death (AICD) via direct DNA-dependent repression of the CD95 ligand gene by a glucocorticoid receptor dimer. Blood $\underline{106}, 617-625$

Becher B, Durell BG, Noelle RJ (2002): Experimental autoimmune encephalitis and inflammation in the absence of interleukin-12. J Clin Invest 110, 493-497

Becher B, Durell BG, Noelle RJ (2003): IL-23 produced by CNS-resident cells controls T cell encephalitogenicity during the effector phase of experimental autoimmune encephalomyelitis. J Clin Invest 112, 1186-1191

Beck RW, Cleary PA, Trobe JD, Kaufman DI, Kupersmith MJ, Paty DW, Brown CH (1993): The effect of corticosteroids for acute optic neuritis on the subsequent development of multiple sclerosis. The Optic Neuritis Study Group. N Engl J Med 329, 1764-1769

Bene NC, Alcaide P, Wortis HH, Jaffe IZ (2014): Mineralocorticoid receptors in immune cells: Emerging role in cardiovascular disease. Steroids $\underline{0}, 38-45$

Ben-Nun A, Wekerle H, Cohen IR (1981): The rapid isolation of clonable antigen-specific T lymphocyte lines capable of mediating autoimmune encephalomyelitis. Eur J Immunol 11, 195-199

Berard JL, Wolak K, Fournier S, David S (2010): Characterization of relapsing-remitting and chronic forms of experimental autoimmune encephalomyelitis in C57BL/6 mice. Glia $\underline{58}$, 434-445

Berger S, Wolfer DP, Selbach O, Alter H, Erdmann G, Reichardt HM, Chepkova AN, Welzl H, Haas HL, Lipp H-P, Schütz G (2006): Loss of the limbic mineralocorticoid receptor impairs behavioral plasticity. Proc Natl Acad Sci U S A $\underline{103}, 195-200$

Bettelli E (2007): Building different mouse models for human MS. Ann N Y Acad Sci $\underline{1103}, 11-18$

Bettelli E, Pagany M, Weiner HL, Linington C, Sobel RA, Kuchroo VK (2003): Myelin Oligodendrocyte Glycoprotein-specific T Cell Receptor Transgenic Mice Develop Spontaneous Autoimmune Optic Neuritis. J Exp Med 197, 1073-1081

Blotta MH, DeKruyff RH, Umetsu DT (1997): Corticosteroids inhibit IL-12 production in human monocytes and enhance their capacity to induce IL-4 synthesis in CD4+ lymphocytes. J Immunol Baltim Md 1950 158, 5589-5595

Boster A, Edan G, Frohman E, Javed A, Stuve O, Tselis A, Weiner H, Weinstock-Guttman B, Khan O, Multiple Sclerosis Clinical Research Center, Department of Neurology, Wayne State University School of Medicine (2008): Intense immunosuppression in patients with rapidly worsening multiple sclerosis: treatment guidelines for the clinician. Lancet Neurol $\underline{7}, 173-183$ 
Braun TP, Marks DL (2015): The regulation of muscle mass by endogenous glucocorticoids. Front Physiol $\underline{6}$

Brennan-Speranza TC, Conigrave AD (2015): Osteocalcin: an osteoblast-derived polypeptide hormone that modulates whole body energy metabolism. Calcif Tissue Int $\underline{96}, 1-10$

Brown JWL, Coles AJ (2013): Alemtuzumab: evidence for its potential in relapsing-remitting multiple sclerosis. Drug Des Devel Ther $\underline{7}, 131-138$

Büyüktimkin B, Wang Q, Kiptoo P, Stewart JM, Berkland C, Siahaan TJ (2012): Vaccine-like controlledrelease delivery of an immunomodulating peptide to treat experimental autoimmune encephalomyelitis. Mol Pharm $\underline{9}, 979-985$

Carlson T, Kroenke M, Rao P, Lane TE, Segal B (2008): The Th17-ELR+ CXC chemokine pathway is essential for the development of central nervous system autoimmune disease. J Exp Med $\underline{205}, 811-823$

Carson MJ, Doose JM, Melchior B, Schmid CD, Ploix CC (2006): CNS immune privilege: hiding in plain sight. Immunol Rev $\underline{213}, 48-65$

Carswell R (1838): Pathological anatomy: illustrations of the elementary forms of disease. Longman, Orme, Brown, Green and Longman (London)

Chandler VL, Maler BA, Yamamoto KR (1983): DNA sequences bound specifically by glucocorticoid receptor in vitro render a heterologous promoter hormone responsive in vivo. Cell $\underline{33}, 489-$ 499

Chantong B, Kratschmar DV, Nashev LG, Balazs Z, Odermatt A (2012): Mineralocorticoid and glucocorticoid receptors differentially regulate NF-kappaB activity and pro-inflammatory cytokine production in murine BV-2 microglial cells. J Neuroinflammation $\underline{9}, 260$

Chao CC, Hu S, Close K, Choi CS, Molitor TW, Novick WJ, Peterson PK (1992): Cytokine release from microglia: differential inhibition by pentoxifylline and dexamethasone. J Infect Dis $\underline{166}, 847-$ 853

Chen X, Winkler-Pickett RT, Carbonetti NH, Ortaldo JR, Oppenheim JJ, Howard OMZ (2006): Pertussis toxin as an adjuvant suppresses the number and function of $\mathrm{CD} 4+\mathrm{CD} 25+\mathrm{T}$ regulatory cells. Eur J Immunol $\underline{36}$, 671-680

Chu CQ, Wittmer S, Dalton DK (2000): Failure to suppress the expansion of the activated CD4 T cell population in interferon gamma-deficient mice leads to exacerbation of experimental autoimmune encephalomyelitis. J Exp Med 192, 123-128

Ciriaco M, Ventrice P, Russo G, Scicchitano M, Mazzitello G, Scicchitano F, Russo E (2013): Corticosteroid-related central nervous system side effects. J Pharmacol Pharmacother $\underline{4}$, S94-S98

Clausen BE, Burkhardt C, Reith W, Renkawitz R, Förster I (1999): Conditional gene targeting in macrophages and granulocytes using LysMcre mice. Transgenic Res $\underline{8}, 265-277$ 
Codarri L, Gyülvészi G, Tosevski V, Hesske L, Fontana A, Magnenat L, Suter T, Becher B (2011): RORyt drives production of the cytokine GM-CSF in helper T cells, which is essential for the effector phase of autoimmune neuroinflammation. Nat Immunol $\underline{12}, 560-567$

Coghlan MJ, Jacobson PB, Lane B, Nakane M, Lin CW, Elmore SW, Kym PR, Luly JR, Carter GW, Turner $R$, et al. (2003): A novel antiinflammatory maintains glucocorticoid efficacy with reduced side effects. Mol Endocrinol Baltim Md 17, 860-869

Compston A, Coles A (2002): Multiple sclerosis. The Lancet $\underline{359}$, 1221-1231

Cua DJ, Sherlock J, Chen Y, Murphy CA, Joyce B, Seymour B, Lucian L, To W, Kwan S, Churakova T, et al. (2003): Interleukin-23 rather than interleukin-12 is the critical cytokine for autoimmune inflammation of the brain. Nature $\underline{421}, 744-748$

D’Adamio F, Zollo O, Moraca R, Ayroldi E, Bruscoli S, Bartoli A, Cannarile L, Migliorati G, Riccardi C (1997): A new dexamethasone-induced gene of the leucine zipper family protects $T$ lymphocytes from TCR/CD3-activated cell death. Immunity $\underline{7}, 803-812$

Demir E, Burgucu D, Turna F, Aksakal S, Kaya B (2013): Determination of TiO2, ZrO2, and Al2O3 nanoparticles on genotoxic responses in human peripheral blood lymphocytes and cultured embyronic kidney cells. J Toxicol Environ Health A 76, 990-1002

Dinkel K, Dhabhar FS, Sapolsky RM (2004): Neurotoxic effects of polymorphonuclear granulocytes on hippocampal primary cultures. Proc Natl Acad Sci U S A 101, 331-336

Duffy SS, Lees JG, Moalem-Taylor G (2014): The Contribution of Immune and Glial Cell Types in Experimental Autoimmune Encephalomyelitis and Multiple Sclerosis. Mult Scler Int 2014

El-Behi M, Ciric B, Dai H, Yan Y, Cullimore M, Safavi F, Zhang G-X, Dittel BN, Rostami A (2011): The encephalitogenicity of $T(H) 17$ cells is dependent on IL-1- and IL-23-induced production of the cytokine GM-CSF. Nat Immunol $\underline{12}$, 568-575

Elkabes S, DiCicco-Bloom EM, Black IB (1996): Brain microglia/macrophages express neurotrophins that selectively regulate microglial proliferation and function. J Neurosci Off J Soc Neurosci $\underline{16}, 2508-2521$

Engelhardt B (2000): Role of glucocorticoids on T cell recruitment across the blood-brain barrier. Z Für Rheumatol 59 Suppl 2, II/18-21

Ehrlich P , Morgenroth J (1957): On Hemolysins: second communication. In The Collected papers of Paul Erlich, vol.2. Immunol and Cancer Research, Ed. Himmelweit 165-172

Ferber IA, Brocke S, Taylor-Edwards C, Ridgway W, Dinisco C, Steinman L, Dalton D, Fathman CG (1996): Mice with a disrupted IFN-gamma gene are susceptible to the induction of experimental autoimmune encephalomyelitis (EAE). J Immunol Baltim Md $1950 \underline{156}$, 5-7

Fernández-Menéndez S, Fernández-Morán M, Fernández-Vega I, Pérez-Álvarez A, Villafani-Echazú J (2016): Epstein-Barr virus and multiple sclerosis. From evidence to therapeutic strategies. J Neurol Sci $\underline{361}, 213-219$ 
Filippini G, Brusaferri F, Sibley WA, Citterio A, Ciucci G, Midgard R, Candelise L (2000): Corticosteroids or ACTH for acute exacerbations in multiple sclerosis. Cochrane Database Syst Rev CD001331

Fischer HJ, Schweingruber N, Lühder F, Reichardt HM (2013): The potential role of T cell migration and chemotaxis as targets of glucocorticoids in multiple sclerosis and experimental autoimmune encephalomyelitis. Mol Cell Endocrinol 380, 99-107

Folgueras AR, Fueyo A, García-Suárez O, Cox J, Astudillo A, Tortorella P, Campestre C, GutiérrezFernández A, Fanjul-Fernández M, Pennington $\mathrm{CJ}$, et al. (2008): Collagenase-2 deficiency or inhibition impairs experimental autoimmune encephalomyelitis in mice. J Biol Chem $\underline{283}$, 9465-9474

Ford ML, Evavold BD (2005): Specificity, magnitude, and kinetics of MOG-specific CD8+ T cell responses during experimental autoimmune encephalomyelitis. Eur J Immunol $\underline{35}$, 76-85

Freund J, Stern ER, Pisani TM (1947): Isoallergic encephalomyelitis and radiculitis in guinea pigs after one injection of brain and Mycobacteria in water-in-oil emulsion. J Immunol Baltim Md 1950 57, 179-194

Funder JW (1993): Mineralocorticoids, glucocorticoids, receptors and response elements. Science $\underline{259}, 1132-1133$

Funder JW (1997): Glucocorticoid and mineralocorticoid receptors: biology and clinical relevance. Annu Rev Med 48, 231-240

van Furth R, Cohn ZA, Hirsch JG, Humphrey JH, Spector WG, Langevoort HL (1972): The mononuclear phagocyte system: a new classification of macrophages, monocytes, and their precursor cells. Bull World Health Organ $\underline{46}, 845-852$

Ganter S, Northoff H, Männel D, Gebicke-Härter PJ (1992): Growth control of cultured microglia. J Neurosci Res $\underline{33}, 218-230$

Gascoyne DM, Kypta RM, Vivanco M d M (2003): Glucocorticoids inhibit apoptosis during fibrosarcoma development by transcriptionally activating Bcl-xL. J Biol Chem 278, 1802218029

Gayo A, Mozo L, Suárez A, Tuñon A, Lahoz C, Gutiérrez C (1998): Glucocorticoids increase IL-10 expression in multiple sclerosis patients with acute relapse. J Neuroimmunol $\underline{85}, 122-130$

Geissmann F, Jung S, Littman DR (2003): Blood monocytes consist of two principal subsets with distinct migratory properties. Immunity $\underline{19}, 71-82$

Giulian D, Baker TJ (1986): Characterization of ameboid microglia isolated from developing mammalian brain. J Neurosci Off J Soc Neurosci $\underline{6}$, 2163-2178

Gold R, Kappos L, Arnold DL, Bar-Or A, Giovannoni G, Selmaj K, Tornatore C, Sweetser MT, Yang M, Sheikh SI, et al. (2012): Placebo-controlled phase 3 study of oral BG-12 for relapsing multiple sclerosis. N Engl J Med 367, 1098-1107

Goodin DS (2014): Glucocorticoid treatment of multiple sclerosis. Handb Clin Neurol $\underline{122}, 455-464$ 
Goodkin DE, Kinkel RP, Weinstock-Guttman B, VanderBrug-Medendorp S, Secic M, Gogol D, Perryman JE, Uccelli MM, Neilley L (1998): A phase II study of i.v. methylprednisolone in secondary-progressive multiple sclerosis. Neurology $\underline{51}, 239-245$

Gordon AN, Fleagle JT, Guthrie D, Parkin DE, Gore ME, Lacave AJ (2001): Recurrent epithelial ovarian carcinoma: a randomized phase III study of pegylated liposomal doxorubicin versus topotecan. J Clin Oncol Off J Am Soc Clin Oncol 19, 3312-3322

Gran B, Zhang G-X, Yu S, Li J, Chen X-H, Ventura ES, Kamoun M, Rostami A (2002): IL-12p35-deficient mice are susceptible to experimental autoimmune encephalomyelitis: evidence for redundancy in the IL-12 system in the induction of central nervous system autoimmune demyelination. J Immunol Baltim Md $1950 \underline{169}, 7104-7110$

Greter M, Heppner FL, Lemos MP, Odermatt BM, Goebels N, Laufer T, Noelle RJ, Becher B (2005): Dendritic cells permit immune invasion of the CNS in an animal model of multiple sclerosis. Nat Med 11, 328-334

Groux H, O’Garra A, Bigler M, Rouleau M, Antonenko S, de Vries JE, Roncarolo MG (1997): A CD4+ Tcell subset inhibits antigen-specific T-cell responses and prevents colitis. Nature $\underline{389}, 737-$ 742

Haak S, Croxford AL, Kreymborg K, Heppner FL, Pouly S, Becher B, Waisman A (2009): IL-17A and IL$17 \mathrm{~F}$ do not contribute vitally to autoimmune neuro-inflammation in mice. J Clin Invest $\underline{119}$, 61-69

Haas J, Hug A, Viehöver A, Fritzsching B, Falk CS, Filser A, Vetter T, Milkova L, Korporal M, Fritz B, et al. (2005): Reduced suppressive effect of CD4+CD25high regulatory $T$ cells on the $T$ cell immune response against myelin oligodendrocyte glycoprotein in patients with multiple sclerosis. Eur J Immunol 35, 3343-3352

Hafler DA, Compston A, Sawcer S, Lander ES, Daly MJ, De Jager PL, de Bakker PIW, Gabriel SB, Mirel DB, Ivinson AJ, et al. (2007): Risk alleles for multiple sclerosis identified by a genomewide study. N Engl J Med 357, 851-862

Harris MG, Hulseberg P, Ling C, Karman J, Clarkson BD, Harding JS, Zhang M, Sandor A, Christensen K, Nagy A, et al. (2014): Immune privilege of the CNS is not the consequence of limited antigen sampling. Sci Rep $\underline{4}, 4422$

Hauser SL, Goodwin DS: Multiple sclerosis and other demyelinating diseases; in: Harrison's Principles of Internal Medicine, Band 2, 17th edition; McGraw-Hill Medical, New York, NY, USA 2008, 2611-2621

Heck JG, Napp J, Simonato S, Möllmer J, Lange M, Reichardt HM, Staudt R, Alves F, Feldmann C (2015): Multifunctional Phosphate-Based Inorganic-Organic Hybrid Nanoparticles. J Am Chem Soc $137,7329-7336$

Hedström AK, Olsson T, Alfredsson L (2015): Smoking is a major preventable risk factor for multiple sclerosis. Mult Scler J 1352458515609794

Hench PS, Kendall EC, Slocumb CH, Polley HF (1950): Cortisone, its effects on rheumatoid arthritis, rheumatic fever, and certain other conditions. Merck Rep $\underline{59}$, 9-14 
Hendriks JJA, Alblas J, van der Pol SMA, van Tol EAF, Dijkstra CD, de Vries HE (2004): Flavonoids influence monocytic GTPase activity and are protective in experimental allergic encephalitis. J Exp Med 200, 1667-1672

Hertzenberg D, Lehmann-Horn K, Kinzel S, Husterer V, Cravens PD, Kieseier BC, Hemmer B, Brück W, Zamvil SS, Stüve O, Weber MS (2013): Developmental maturation of innate immune cell function correlates with susceptibility to central nervous system autoimmunity. Eur J Immunol 43, 2078-2088

Hirota K, Duarte JH, Veldhoen M, Hornsby E, Li Y, Cua DJ, Ahlfors H, Wilhelm C, Tolaini M, Menzel U, et al. (2011): Fate mapping of IL-17-producing T cells in inflammatory responses. Nat Immunol 12, 255-263

Hodge S, Hodge G, Flower R, Han P (1999): Methyl-prednisolone up-regulates monocyte interleukin10 production in stimulated whole blood. Scand J Immunol 49, 548-553

Hou W, Wu Y, Sun S, Shi M, Sun Y, Yang C, Pei G, Gu Y, Zhong C, Sun B (2003): Pertussis toxin enhances Th1 responses by stimulation of dendritic cells. J Immunol Baltim Md $1950 \underline{170}$, 1728-1736

Hu X, Liu G, Hou Y, Shi J, Zhu L, Jin D, Peng J, Zhao Y (2012): Induction of M2-like macrophages in recipient NOD-scid mice by allogeneic donor $\mathrm{CD} 4(+) \mathrm{CD} 25(+)$ regulatory $\mathrm{T}$ cells. Cell Mol Immunol $\underline{9}$, 464-472

Huang W, Metlakunta A, Dedousis N, Zhang P, Sipula I, Dube JJ, Scott DK, O'Doherty RM (2010): Depletion of liver Kupffer cells prevents the development of diet-induced hepatic steatosis and insulin resistance. Diabetes $\underline{59}, 347-357$

Hucke S, Eschborn M, Liebmann M, Herold M, Freise N, Engbers A, Ehling P, Meuth SG, Roth J, Kuhlmann T, et al. (2016): Sodium chloride promotes pro-inflammatory macrophage polarization thereby aggravating CNS autoimmunity. J Autoimmun $\underline{67}$, 90-101

Huitinga I, van Rooijen N, de Groot CJ, Uitdehaag BM, Dijkstra CD (1990): Suppression of experimental allergic encephalomyelitis in Lewis rats after elimination of macrophages. J Exp Med 172, 1025-1033

Hume DA (2015): The Many Alternative Faces of Macrophage Activation. Front Immunol $\underline{6}, 370$

Hunger M, Budinger E, Zhong K, Angenstein F (2014): Visualization of acute focal lesions in rats with experimental autoimmune encephalomyelitis by magnetic nanoparticles, comparing different MRI sequences including phase imaging. J Magn Reson Imaging $\underline{39}$, 1126-1135

Hunter RW, Ivy JR, Bailey MA (2014a): Glucocorticoids and renal Na+ transport: implications for hypertension and salt sensitivity. J Physiol 592, 1731-1744

Hunter Z, McCarthy DP, Yap WT, Harp CT, Getts DR, Shea LD, Miller SD (2014b): A biodegradable nanoparticle platform for the induction of antigen-specific immune tolerance for treatment of autoimmune disease. ACS Nano $\underline{8}, 2148-2160$

Huppert J, Closhen D, Croxford A, White R, Kulig P, Pietrowski E, Bechmann I, Becher B, Luhmann HJ, Waisman A, Kuhlmann CRW (2010): Cellular mechanisms of IL-17-induced blood-brain barrier disruption. FASEB J Off Publ Fed Am Soc Exp Biol 24, 1023-1034 
Hutchison KA, Dittmar KD, Stancato LF, Pratt WB (1996): Ability of various members of the hsp70 family of chaperones to promote assembly of the glucocorticoid receptor into a functional heterocomplex with hsp90. J Steroid Biochem Mol Biol $\underline{58}$, 251-258

Hwang JL, Weiss RE (2014): Steroid-induced diabetes: a clinical and molecular approach to understanding and treatment. Diabetes Metab Res Rev $\underline{30}$, 96-102

Iliff JJ, Goldman SA, Nedergaard M (2015): Implications of the discovery of brain lymphatic pathways. Lancet Neurol 14, 977-979

Imrich H, Harzer K (2001): On the role of peripheral macrophages during active experimental allergic encephalomyelitis (EAE). J Neural Transm Vienna Austria 1996 108, 379-395

Johnson KP, Brooks BR, Cohen JA, Ford CC, Goldstein J, Lisak RP, Myers LW, Panitch HS, Rose JW, Schiffer RB (1995): Copolymer 1 reduces relapse rate and improves disability in relapsingremitting multiple sclerosis: results of a phase III multicenter, double-blind placebocontrolled trial. The Copolymer 1 Multiple Sclerosis Study Group. Neurology $4 \underline{5}, 1268-1276$

Jun CD, Hoon-Ryu null, Um JY, Kim TY, Kim JM, Kang SS, Kim HM, Chung HT (1994): Involvement of protein kinase $C$ in the inhibition of nitric oxide production from murine microglial cells by glucocorticoid. Biochem Biophys Res Commun 199, 633-638

Kappos L, Radue E-W, O'Connor P, Polman C, Hohlfeld R, Calabresi P, Selmaj K, Agoropoulou C, Leyk $M$, Zhang-Auberson $L$, et al. (2010): A placebo-controlled trial of oral fingolimod in relapsing multiple sclerosis. N Engl J Med $\underline{362}$, 387-401

Karunakaran G, Suriyaprabha R, Manivasakan P, Yuvakkumar R, Rajendran V, Kannan N (2013): Screening of in vitro cytotoxicity, antioxidant potential and bioactivity of nano- and microZrO2 and -TiO2 particles. Ecotoxicol Environ Saf 93, 191-197

Kashiwamura Y, Sano Y, Abe M, Shimizu F, Haruki H, Maeda T, Kawai M, Kanda T (2011): Hydrocortisone enhances the function of the blood-nerve barrier through the up-regulation of claudin-5. Neurochem Res $\underline{36}$, 849-855

Kassiotis G, Pasparakis M, Kollias G, Probert L (1999): TNF accelerates the onset but does not alter the incidence and severity of myelin basic protein-induced experimental autoimmune encephalomyelitis. Eur J Immunol $\underline{29}, 774-780$

Keating P, O'Sullivan D, Tierney JB, Kenwright D, Miromoeini S, Mawasse L, Brombacher F, La Flamme AC (2009): Protection from EAE by IL-4Ralpha(-/-) macrophages depends upon T regulatory cell involvement. Immunol Cell Biol 87, 534-545

Kerfoot SM, Long EM, Hickey MJ, Andonegui G, Lapointe BM, Zanardo RCO, Bonder C, James WG, Robbins SM, Kubes P (2004): TLR4 contributes to disease-inducing mechanisms resulting in central nervous system autoimmune disease. J Immunol Baltim Md $1950 \underline{173}$, 7070-7077

Kiefer R, Kreutzberg GW (1991): Effects of dexamethasone on microglial activation in vivo: selective downregulation of major histocompatibility complex class II expression in regenerating facial nucleus. J Neuroimmunol 34, 99-108

Kierdorf K, Prinz M (2013): Factors regulating microglia activation. Front Cell Neurosci $\underline{7}, 44$ 
Kizelsztein P, Ovadia H, Garbuzenko O, Sigal A, Barenholz Y (2009): Pegylated nanoliposomes remoteloaded with the antioxidant tempamine ameliorate experimental autoimmune encephalomyelitis. J Neuroimmunol $\underline{213}, 20-25$

Kleiman A, Hübner S, Rodriguez Parkitna JM, Neumann A, Hofer S, Weigand MA, Bauer M, Schmid W, Schütz G, Libert C, et al. (2012): Glucocorticoid receptor dimerization is required for survival in septic shock via suppression of interleukin-1 in macrophages. FASEB J Off Publ Fed Am Soc Exp Biol 26, 722-729

Kleinewietfeld M, Manzel A, Titze J, Kvakan H, Yosef N, Linker RA, Muller DN, Hafler DA (2013): Sodium Chloride Drives Autoimmune Disease by the Induction of Pathogenic Th17 Cells. Nature $\underline{496}, 518-522$

Koenen HJPM, Smeets RL, Vink PM, van Rijssen E, Boots AMH, Joosten I (2008): Human CD25highFoxp3pos regulatory T cells differentiate into IL-17-producing cells. Blood $\underline{112}$, 2340-2352

Koh DR, Fung-Leung WP, Ho A, Gray D, Acha-Orbea H, Mak TW (1992): Less mortality but more relapses in experimental allergic encephalomyelitis in CD8-/- mice. Science $\underline{256}, 1210-1213$

Kohm AP, Carpentier PA, Miller SD (2003): Regulation of experimental autoimmune encephalomyelitis (EAE) by CD4+CD25+ regulatory T cells. Novartis Found Symp 252, 45-52$54,106-114$

Komiyama Y, Nakae S, Matsuki T, Nambu A, Ishigame H, Kakuta S, Sudo K, Iwakura Y (2006): IL-17 plays an important role in the development of experimental autoimmune encephalomyelitis. J Immunol Baltim Md $1950 \underline{177}$, 566-573

Korn T, Oukka M, Kuchroo V, Bettelli E (2007a): Th17 cells: effector T cells with inflammatory properties. Semin Immunol 19, 362-371

Korn T, Anderson AC, Bettelli E, Oukka M (2007b): The dynamics of effector T cells and Foxp3+ regulatory $T$ cells in the promotion and regulation of autoimmune encephalitis. $J$ Neuroimmunol $\underline{191}, 51-60$

Korn T, Bettelli E, Oukka M, Kuchroo VK (2009): IL-17 and Th17 Cells. Annu Rev Immunol 27, 485-517

Körner H, Riminton DS, Strickland DH, Lemckert FA, Pollard JD, Sedgwick JD (1997): Critical points of tumor necrosis factor action in central nervous system autoimmune inflammation defined by gene targeting. J Exp Med 186, 1585-1590

Kotter MR, Li W, Franklin RJM (2006): Myelin impairs CNS remyelination by inhibiting oligondendrocyte precursor cell differentiation. J Neurosci 26(1), 328-332

Kraaij MD, Savage NDL, van der Kooij SW, Koekkoek K, Wang J, van den Berg JM, Ottenhoff THM, Kuijpers TW, Holmdahl R, van Kooten C, Gelderman KA (2010): Induction of regulatory T cells by macrophages is dependent on production of reactive oxygen species. Proc Natl Acad Sci U S A $\underline{107}, 17686-17691$ 
Krakowski M, Owens T (1996): Interferon-gamma confers resistance to experimental allergic encephalomyelitis. Eur J Immunol 26, 1641-1646

Kroenke MA, Segal BM (2011): IL-23 modulated myelin-specific T cells induce EAE via an IFNY driven, IL-17 independent pathway. Brain Behav Immun 25, 932-937

Kuo T, McQueen A, Chen T-C, Wang J-C (2015): Regulation of Glucose Homeostasis by Glucocorticoids. Adv Exp Med Biol $\underline{872}$, 99-126

Kurschus FC, Croxford AL, Heinen AP, Wörtge S, lelo D, Waisman A (2010): Genetic proof for the transient nature of the Th17 phenotype. Eur J Immunol $\underline{40}$, 3336-3346

Lafaille JJ, Keere FV, Hsu AL, Baron JL, Haas W, Raine CS, Tonegawa S (1997): Myelin basic proteinspecific $T$ helper 2 (Th2) cells cause experimental autoimmune encephalomyelitis in immunodeficient hosts rather than protect them from the disease. J Exp Med 186, 307-312

Langrish CL, Chen Y, Blumenschein WM, Mattson J, Basham B, Sedgwick JD, McClanahan T, Kastelein RA, Cua DJ (2005): IL-23 drives a pathogenic T cell population that induces autoimmune inflammation. J Exp Med 201, 233-240

Lassmann H, Brück W, Lucchinetti CF (2007): The Immunopathology of Multiple Sclerosis: An Overview. Brain Pathol 17, 210-218

Leclerc N, Noh T, Khokhar A, Smith E, Frenkel B (2005): Glucocorticoids inhibit osteocalcin transcription in osteoblasts by suppressing Egr2/Krox20-binding enhancer. Arthritis Rheum 52, 929-939

Leray E, Moreau T, Fromont A, Edan G (2016): Epidemiology of multiple sclerosis. Rev Neurol (Paris) $172,3-13$

Leussink VI, Jung S, Merschdorf U, Toyka KV, Gold R (2001): High-dose methylprednisolone therapy in multiple sclerosis induces apoptosis in peripheral blood leukocytes. Arch Neurol 58, 91-97

Li X (2013): Myeloid cell-specific ablation of the mineralocorticoid receptor attenuates experimental autoimmune encephalomyelitis. http://hdl.handle.net/11858/00-1735-0000-000D-FB53-E

Lim H-Y, Müller N, Herold MJ, van den Brandt J, Reichardt HM (2007): Glucocorticoids exert opposing effects on macrophage function dependent on their concentration. Immunology 122, 47-53

Linker RA, Weller C, Lühder F, Mohr A, Schmidt J, Knauth M, Metselaar JM, Gold R (2008): Liposomal glucocorticosteroids in treatment of chronic autoimmune demyelination: long-term protective effects and enhanced efficacy of methylprednisolone formulations. Exp Neurol 211, 397-406

Litchfield S, Nagy Z (2001): New temperature modification makes the Bielschowsky silver stain reproducible. Acta Neuropathol (Berl) 101, 17-21

Litzenburger T, Fässler R, Bauer J, Lassmann H, Linington C, Wekerle H, Iglesias A (1998): B lymphocytes producing demyelinating autoantibodies: development and function in genetargeted transgenic mice. J Exp Med 188, 169-180 
Liu Y, Teige I, Birnir B, Issazadeh-Navikas S (2006): Neuron-mediated generation of regulatory T cells from encephalitogenic T cells suppresses EAE. Nat Med 12, 518-525

Louw A, Swart P, de Kock SS, van der Merwe KJ (1997): Mechanism for the stabilization in vivo of the aziridine precursor --(4-acetoxyphenyl)-2-chloro-N-methyl-ethylammonium chloride by

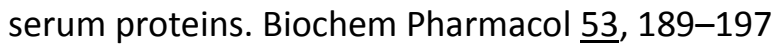

Lublin FD, Maurer PH, Berry RG, Tippett D (1981): Delayed, relapsing experimental allergic encephalomyelitis in mice. J Immunol Baltim Md $1950 \underline{126}$, 819-822

Lucchinetti C, Brück W, Parisi J, Scheithauer B, Rodriguez M, Lassmann H (2000): Heterogeneity of multiple sclerosis lesions: implications for the pathogenesis of demyelination. Ann Neurol $\underline{47}$, 707-717

Luche H, Weber O, Nageswara Rao T, Blum C, Fehling HJ (2007): Faithful activation of an extra-bright red fluorescent protein in "knock-in“ Cre-reporter mice ideally suited for lineage tracing studies. Eur J Immunol 37, 43-53

Mantovani A, Sica A, Sozzani S, Allavena P, Vecchi A, Locati M (2004): The chemokine system in diverse forms of macrophage activation and polarization. Trends Immunol $\underline{25}, 677-686$

Markus MA, Napp J, Behnke T, Mitkovski M, Monecke S, Dullin C, Kilfeather S, Dressel R, ReschGenger U, Alves F (2015): Tracking of Inhaled Near-Infrared Fluorescent Nanoparticles in Lungs of SKH-1 Mice with Allergic Airway Inflammation. ACS Nano $\underline{9}, 11642-11657$

Martinez FO, Gordon S (2014): The M1 and M2 paradigm of macrophage activation: time for reassessment. F1000Prime Rep $\underline{6}, 13$

Matsushima GK, Morell P (2001): The neurotoxicant, cuprizone, as a model to study demyelination and remyelination in the central nervous system. Brain Pathol Zurich Switz 11, 107-116

McColl SR, Staykova MA, Wozniak A, Fordham S, Bruce J, Willenborg DO (1998): Treatment with antigranulocyte antibodies inhibits the effector phase of experimental autoimmune encephalomyelitis. J Immunol Baltim Md $1950 \underline{161}$, 6421-6426

McDonald WI, Compston A, Edan G, Goodkin D, Hartung HP, Lublin FD, McFarland HF, Paty DW, Polman CH, Reingold SC, et al. (2001): Recommended diagnostic criteria for multiple sclerosis: guidelines from the International Panel on the diagnosis of multiple sclerosis. Ann Neurol $\underline{50}, 121-127$

McGeachy MJ, Stephens LA, Anderton SM (2005): Natural recovery and protection from autoimmune encephalomyelitis: contribution of CD4+CD25+ regulatory cells within the central nervous system. J Immunol Baltim Md $1950 \underline{175}$, 3025-3032

Medawar PB (1948): Immunity to Homologous Grafted Skin. III. The Fate of Skin Homographs Transplanted to the Brain, to Subcutaneous Tissue, and to the Anterior Chamber of the Eye. Br J Exp Pathol 29, 58-69

Merodio M, Irache JM, Eclancher F, Mirshahi M, Villarroya H (2000): Distribution of albumin nanoparticles in animals induced with the experimental allergic encephalomyelitis. J Drug Target $\underline{8}, 289-303$ 
Metselaar JM, Wauben MHM, Wagenaar-Hilbers JPA, Boerman OC, Storm G (2003): Complete remission of experimental arthritis by joint targeting of glucocorticoids with long-circulating liposomes. Arthritis Rheum 48, 2059-2066

Mikita J, Dubourdieu-Cassagno N, Deloire MS, Vekris A, Biran M, Raffard G, Brochet B, Canron M-H, Franconi J-M, Boiziau C, Petry KG (2011): Altered M1/M2 activation patterns of monocytes in severe relapsing experimental rat model of multiple sclerosis. Amelioration of clinical status by $\mathrm{M} 2$ activated monocyte administration. Mult Scler J $\underline{17}, 2-15$

Mildner A, Mack M, Schmidt H, Brück W, Djukic M, Zabel MD, Hille A, Priller J, Prinz M (2009): CCR2+Ly-6Chi monocytes are crucial for the effector phase of autoimmunity in the central nervous system. Brain J Neurol 132, 2487-2500

Miller DM, Weinstock-Guttman B, Béthoux F, Lee JC, Beck G, Block V, Durelli L, LaMantia L, Barnes D, Sellebjerg F, Rudick RA (2000): A meta-analysis of methylprednisolone in recovery from multiple sclerosis exacerbations. Mult Scler Houndmills Basingstoke Engl $\underline{6}, 267-273$

Miller H, Newell DJ, Ridley A (1961): Multiple sclerosis. Trials of maintenance treatment with prednisolone and soluble aspirin. Lancet Lond Engl $\underline{1}$, 127-129

Milligan NM, Newcombe R, Compston DA (1987): A double-blind controlled trial of high dose methylprednisolone in patients with multiple sclerosis: 1 . Clinical effects. J Neurol Neurosurg Psychiatry $\underline{50}, 511-516$

Mimura LAN, Chiuso-Minicucci F, Fraga-Silva TFC, Zorzella-Pezavento S. G, Franca TG., Ishikawa LLW, Penitenti M, Ikoma MRV, Sartori A (2016): Association of myelin peptide with vitamin D prevents autoimmune encephalomyelitis development. Neuroscience $\underline{317}, 130-140$

Moghadam-Kia S, Werth VP (2010): Prevention and treatment of systemic glucocorticoid side effects. Int J Dermatol 49, 239-248

Mosmann TR, Cherwinski H, Bond MW, Giedlin MA, Coffman RL (1986): Two types of murine helper T cell clone. I. Definition according to profiles of lymphokine activities and secreted proteins. J Immunol Baltim Md 1950 136, 2348-2357

Mosser DM, Edwards JP (2008): Exploring the full spectrum of macrophage activation. Nat Rev Immunol $\underline{8}, 958-969$

Murphy AC, Lalor SJ, Lynch MA, Mills KHG (2010): Infiltration of Th1 and Th17 cells and activation of microglia in the CNS during the course of experimental autoimmune encephalomyelitis. Brain Behav Immun 24, 641-651

Neumann H, Kotter MR, Franklin RJM (2009): Debris clearance by microglia: an essential link between degeneration and regeneration. Brain J Neurol 132, 288-295

Obando-Pereda GA, Fischer L, Stach-Machado DR (2014): Titanium and zirconia particle-induced proinflammatory gene expression in cultured macrophages and osteolysis, inflammatory

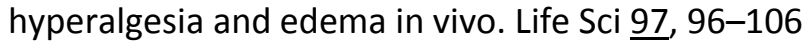

O'Connor RA, Malpass KH, Anderton SM (2007): The inflamed central nervous system drives the activation and rapid proliferation of Foxp3+ regulatory T cells. J Immunol Baltim Md 1950 $\underline{179}, 958-966$ 
Odegaard JI, Ricardo-Gonzalez RR, Red Eagle A, Vats D, Morel CR, Goforth MH, Subramanian V, Mukundan L, Ferrante AW, Chawla A (2008): Alternative M2 activation of Kupffer cells by PPARdelta ameliorates obesity-induced insulin resistance. Cell Metab $\underline{7}, 496-507$

Oppmann B, Lesley R, Blom B, Timans JC, Xu Y, Hunte B, Vega F, Yu N, Wang J, Singh K, et al. (2000): Novel p19 protein engages IL-12p40 to form a cytokine, IL-23, with biological activities similar as well as distinct from IL-12. Immunity $\underline{13}, 715-725$

Panitch HS, Hirsch RL, Schindler J, Johnson KP (1987): Treatment of multiple sclerosis with gamma interferon: exacerbations associated with activation of the immune system. Neurology $\underline{37}$, 1097-1102

Papadopoulou A, Kappos L, Sprenger T (2012): Teriflunomide for oral therapy in multiple sclerosis. Expert Rev Clin Pharmacol $\underline{5}, 617-628$

Paty DW, Li DK (1993): Interferon beta-1b is effective in relapsing-remitting multiple sclerosis. II. MRI analysis results of a multicenter, randomized, double-blind, placebo-controlled trial. UBC MS/MRI Study Group and the IFNB Multiple Sclerosis Study Group. Neurology 43, 662-667

Paul C, Bolton C (1995): Inhibition of blood-brain barrier disruption in experimental allergic encephalomyelitis by short-term therapy with dexamethasone or cyclosporin A. Int J Immunopharmacol 17, 497-503

Pitt B, Zannad F, Remme WJ, Cody R, Castaigne A, Perez A, Palensky J, Wittes J (1999): The effect of spironolactone on morbidity and mortality in patients with severe heart failure. Randomized Aldactone Evaluation Study Investigators. N Engl J Med 341, 709-717

Pitzalis C, Pipitone N, Perretti M (2002): Regulation of leukocyte-endothelial interactions by glucocorticoids. Ann N Y Acad Sci $\underline{966}, 108-118$

Polman CH, O'Connor PW, Havrdova E, Hutchinson M, Kappos L, Miller DH, Phillips JT, Lublin FD, Giovannoni G, Wajgt A, et al. (2006): A randomized, placebo-controlled trial of natalizumab for relapsing multiple sclerosis. N Engl J Med 354, 899-910

Prinz M, Schmidt H, Mildner A, Knobeloch K-P, Hanisch U-K, Raasch J, Merkler D, Detje C, Gutcher I, Mages J, et al. (2008): Distinct and nonredundant in vivo functions of IFNAR on myeloid cells limit autoimmunity in the central nervous system. Immunity $\underline{28}, 675-686$

Prinz M, Priller J, Sisodia SS, Ransohoff RM (2011): Heterogeneity of CNS myeloid cells and their roles in neurodegeneration. Nat Neurosci 14, 1227-1235

Procaccini C, De Rosa V, Pucino V, Formisano L, Matarese G (2015): Animal models of Multiple Sclerosis. Eur J Pharmacol $\underline{759}, 182-191$

Rauch A, Seitz S, Baschant U, Schilling AF, Illing A, Stride B, Kirilov M, Mandic V, Takacz A, SchmidtUllrich R, et al. (2010): Glucocorticoids suppress bone formation by attenuating osteoblast differentiation via the monomeric glucocorticoid receptor. Cell Metab 11, 517-531

Rausch M, Hiestand P, Foster CA, Baumann DR, Cannet C, Rudin M (2004): Predictability of FTY720 efficacy in experimental autoimmune encephalomyelitis by in vivo macrophage tracking: Clinical implications for ultrasmall superparamagnetic iron oxide-enhanced magnetic resonance imaging. J Magn Reson Imaging 20, 16-24 
Ravnborg M, Sørensen PS, Andersson M, Celius EG, Jongen PJ, Elovaara I, Bartholomé E, Constantinescu CS, Beer K, Garde E, Sperling B (2010): Methylprednisolone in combination with interferon beta-1a for relapsing-remitting multiple sclerosis (MECOMBIN study): a multicentre, double-blind, randomised, placebo-controlled, parallel-group trial. Lancet Neurol $\underline{9}, 672-680$

Reichardt HM, Kaestner KH, Tuckermann J, Kretz O, Wessely O, Bock R, Gass P, Schmid W, Herrlich P, Angel P, Schütz G (1998): DNA binding of the glucocorticoid receptor is not essential for survival. Cell $\underline{93}, 531-541$

Reichardt SD, Föller M, Rexhepaj R, Pathare G, Minnich K, Tuckermann JP, Lang F, Reichardt HM (2012): Glucocorticoids enhance intestinal glucose uptake via the dimerized glucocorticoid receptor in enterocytes. Endocrinology 153, 1783-1794

Reichardt SD, Weinhage T, Rotte A, Föller M, Oppermann M, Lühder F, Tuckermann JP, Lang F, van den Brandt J, Reichardt HM (2014): Glucocorticoids induce gastroparesis in mice through depletion of I-arginine. Endocrinology 155, 3899-3908

Renno T, Zeine R, Girard JM, Gillani S, Dodelet V, Owens T (1994): Selective enrichment of Th1 CD45RBlow CD4+ $T$ cells in autoimmune infiltrates in experimental allergic encephalomyelitis. Int Immunol $\underline{6}$, 347-354

Renoux C (2011): Natural history of multiple sclerosis: long-term prognostic factors. Neurol Clin $\underline{29}$, 293-308

van Rensburg K (2011): A guide to prescribing corticosteroids. SA Pharm J $\underline{78}, 32-38$

Reynolds A, Laurie C, Mosley RL, Gendelman HE (2007): Oxidative stress and the pathogenesis of neurodegenerative disorders. Int Rev Neurobiol 82, 297-325

Rhen T, Cidlowski JA (2005): Antiinflammatory Action of Glucocorticoids - New Mechanisms for Old Drugs. N Engl J Med $\underline{353}, 1711-1723$

Rickard AJ, Morgan J, Tesch G, Funder JW, Fuller PJ, Young MJ (2009): Deletion of mineralocorticoid receptors from macrophages protects against deoxycorticosterone/salt-induced cardiac fibrosis and increased blood pressure. Hypertension $\underline{54}, 537-543$

Ridder DA, Lang M-F, Salinin S, Röderer J-P, Struss M, Maser-Gluth C, Schwaninger M (2011): TAK1 in brain endothelial cells mediates fever and lethargy. J Exp Med 208, 2615-2623

Rivers TM, Sprunt DH, Berry GP (1933): Observations on attempts to produce acute disseminated encephalomyelitis in monkeys. J Exp Med $\underline{58}, 39-53$

Rose S, Misharin A, Perlman H (2012): A novel Ly6C/Ly6G-based strategy to analyze the mouse splenic myeloid compartment. Cytom Part J Int Soc Anal Cytol 81, 343-350

Rosenberg GA, Dencoff JE, Correa N, Reiners M, Ford CC (1996): Effect of steroids on CSF matrix metalloproteinases in multiple sclerosis: relation to blood-brain barrier injury. Neurology $\underline{46}$, 1626-1632

Rőszer T (2015): Understanding the Mysterious M2 Macrophage through Activation Markers and Effector Mechanisms. Mediators Inflamm 2015, 816460 
Sadovnick AD (1993): Familial recurrence risks and inheritance of multiple sclerosis. Curr Opin Neurol Neurosurg 6 , 189-194

Sakaguchi S, Yamaguchi T, Nomura T, Ono M (2008): Regulatory T cells and immune tolerance. Cell $\underline{133}, 775-787$

Sakai DD, Helms S, Carlstedt-Duke J, Gustafsson JA, Rottman FM, Yamamoto KR (1988): Hormonemediated repression: a negative glucocorticoid response element from the bovine prolactin gene. Genes Dev $\underline{2}, 1144-1154$

Savage NDL, de Boer T, Walburg KV, Joosten SA, van Meijgaarden K, Geluk A, Ottenhoff THM (2008): Human anti-inflammatory macrophages induce Foxp3+ GITR+CD25+ regulatory $T$ cells, which suppress via membrane-bound TGFbeta-1. J Immunol Baltim Md 1950 181, 2220-2226

Sawcer S, Hellenthal G, Pirinen M, Spencer CCA, Patsopoulos NA, Moutsianas L, Dilthey A, Su Z, Freeman C, Hunt SE, et al. (2011): Genetic risk and a primary role for cell-mediated immune mechanisms in multiple sclerosis. Nature $\underline{476}, 214-219$

Saxena V, Diaz A, Clearfield A, Batteas JD, Hussain MD (2013): Zirconium phosphate nanoplatelets: a biocompatible nanomaterial for drug delivery to cancer. Nanoscale $\underline{5}, 2328-2336$

Schakman O, Kalista S, Barbé C, Loumaye A, Thissen JP (2013): Glucocorticoid-induced skeletal muscle atrophy. Int J Biochem Cell Biol $\underline{45}, 2163-2172$

Schmidt J, Metselaar JM, Gold R (2003): Intravenous liposomal prednisolone downregulates in situ TNF-alpha production by T-cells in experimental autoimmune encephalomyelitis. J Histochem Cytochem Off J Histochem Soc $\underline{51}, 1241-1244$

Schweingruber N, Haine A, Tiede K, Karabinskaya A, van den Brandt J, Wüst S, Metselaar JM, Gold R, Tuckermann JP, Reichardt HM, Lühder F (2011): Liposomal encapsulation of glucocorticoids alters their mode of action in the treatment of experimental autoimmune encephalomyelitis. J Immunol Baltim Md $1950 \underline{187}$, 4310-4318

Schweingruber N, Fischer HJ, Fischer L, van den Brandt J, Karabinskaya A, Labi V, Villunger A, Kretzschmar B, Huppke P, Simons M, et al. (2014): Chemokine-mediated redirection of T cells constitutes a critical mechanism of glucocorticoid therapy in autoimmune CNS responses. Acta Neuropathol (Berl) $\underline{127}, 713-729$

Shijie J, Takeuchi H, Yawata I, Harada Y, Sonobe Y, Doi Y, Liang J, Hua L, Yasuoka S, Zhou Y, et al. (2009): Blockade of glutamate release from microglia attenuates experimental autoimmune encephalomyelitis in mice. Tohoku J Exp Med 217, 87-92

Skurkovich S, Boiko A, Beliaeva I, Buglak A, Alekseeva T, Smirnova N, Kulakova O, Tchechonin V, Gurova O, Deomina T, et al. (2001): Randomized study of antibodies to IFN-gamma and TNFalpha in secondary progressive multiple sclerosis. Mult Scler Houndmills Basingstoke Engl $\underline{7}$, 277-284

Smith AJ, Liu Y, Peng H, Beers R, Racke MK, Lovett-Racke AE (2011): Comparison of a classical Th1 bacteria versus a Th17 bacteria as adjuvant in the induction of experimental autoimmune encephalomyelitis. J Neuroimmunol 237, 33-38 
Sorensen PS, Mellgren SI, Svenningsson A, Elovaara I, Frederiksen JL, Beiske AG, Myhr K-M, Søgaard LV, Olsen IC, Sandberg-Wollheim M (2009): NORdic trial of oral Methylprednisolone as addon therapy to Interferon beta-1a for treatment of relapsing-remitting Multiple Sclerosis (NORMIMS study): a randomised, placebo-controlled trial. Lancet Neurol $\underline{8}, 519-529$

Soulika AM, Lee E, McCauley E, Miers L, Bannerman P, Pleasure D (2009): Initiation and progression of axonopathy in experimental autoimmune encephalomyelitis. J Neurosci Off J Soc Neurosci $\underline{29}, 14965-14979$

Steinbach K, Piedavent M, Bauer S, Neumann JT, Friese MA (2013): Neutrophils amplify autoimmune central nervous system infiltrates by maturing local APCs. J Immunol Baltim Md $1950 \underline{191}$, 4531-4539

Steinbuch M, Youket TE, Cohen S (2004): Oral glucocorticoid use is associated with an increased risk of fracture. Osteoporos Int J Establ Result Coop Eur Found Osteoporos Natl Osteoporos Found USA $\underline{15}, 323-328$

Strehl C, Gaber T, Löwenberg M, Hommes DW, Verhaar AP, Schellmann S, Hahne M, Fangradt M, Wagegg $M$, Hoff $P$, et al. (2011): Origin and functional activity of the membrane-bound glucocorticoid receptor. Arthritis Rheum 63 , 3779-3788

Stromnes IM, Cerretti LM, Liggitt D, Harris RA, Goverman JM (2008): Differential regulation of central nervous system autoimmunity by $\mathrm{T}(\mathrm{H}) 1$ and $\mathrm{T}(\mathrm{H}) 17$ cells. Nat Med $\underline{14}, 337-342$

Strömstedt PE, Poellinger L, Gustafsson JA, Carlstedt-Duke J (1991): The glucocorticoid receptor binds to a sequence overlapping the TATA box of the human osteocalcin promoter: a potential mechanism for negative regulation. Mol Cell Biol $\underline{11}, 3379-3383$

Svensson-Arvelund J, Mehta RB, Lindau R, Mirrasekhian E, Rodriguez-Martinez H, Berg G, Lash GE, Jenmalm MC, Ernerudh J (2015): The human fetal placenta promotes tolerance against the semiallogeneic fetus by inducing regulatory $\mathrm{T}$ cells and homeostatic M2 macrophages. J Immunol Baltim Md 1950 194, 1534-1544

Tabansky I, Messina MD, Bangeranye C, Goldstein J, Blitz-Shabbir KM, Machado S, Jeganathan V, Wright P, Najjar S, Cao Y, et al. (2015): Advancing drug delivery systems for the treatment of multiple sclerosis. Immunol Res $\underline{63}, 58-69$

Tahover E, Patil YP, Gabizon AA (2015): Emerging delivery systems to reduce doxorubicin cardiotoxicity and improve therapeutic index: focus on liposomes. Anticancer Drugs $\underline{26}, 241-$ 258

Tanaka J, Fujita H, Matsuda S, Toku K, Sakanaka M, Maeda N (1997): Glucocorticoid- and mineralocorticoid receptors in microglial cells: The two receptors mediate differential effects of corticosteroids. Glia $\underline{20}, 23-37$

Then Bergh F, Kümpfel T, Schumann E, Held U, Schwan M, Blazevic M, Wismüller A, Holsboer F, Yassouridis A, Uhr M, et al. (2006): Monthly intravenous methylprednisolone in relapsingremitting multiple sclerosis - reduction of enhancing lesions, T2 lesion volume and plasma prolactin concentrations. BMC Neurol $\underline{6}, 19$

Thornton AM, Shevach EM (2000): Suppressor effector function of CD4+CD25+ immunoregulatory $T$ cells is antigen nonspecific. J Immunol Baltim Md $1950 \underline{164}$, 183-190 
Tischner D, Weishaupt A, van den Brandt J, Müller N, Beyersdorf N, Ip CW, Toyka KV, Hünig T, Gold R, Kerkau T, Reichardt HM (2006): Polyclonal expansion of regulatory T cells interferes with effector cell migration in a model of multiple sclerosis. Brain J Neurol $\underline{129}, 2635-2647$

Toft-Hansen H, Nuttall RK, Edwards DR, Owens T (2004): Key metalloproteinases are expressed by specific cell types in experimental autoimmune encephalomyelitis. J Immunol Baltim Md $1950 \underline{173}, 5209-5218$

Tourtellotte WW, Haerer AF (1965): Use of an oral corticosteroid in the treatment of multiple sclerosis; a double-blind study. Arch Neurol 12, 536-545

Traboulsee AL, Bernales CQ, Ross JP, Lee JD, Sadovnick AD, Vilariño-Güell C (2014): Genetic variants in IL2RA and IL7R affect multiple sclerosis disease risk and progression. Neurogenetics $\underline{15}$, 165-169

Tran EH, Hoekstra K, van Rooijen N, Dijkstra CD, Owens T (1998): Immune invasion of the central nervous system parenchyma and experimental allergic encephalomyelitis, but not leukocyte extravasation from blood, are prevented in macrophage-depleted mice. J Immunol Baltim Md $1950 \underline{161}, 3767-3775$

Tronche F, Kellendonk C, Kretz O, Gass P, Anlag K, Orban PC, Bock R, Klein R, Schütz G (1999): Disruption of the glucocorticoid receptor gene in the nervous system results in reduced anxiety. Nat Genet 23, 99-103

Tsunoda I, Libbey JE, Kobayashi-Warren M, Fujinami RS (2006): IFN- $\gamma$ production and astrocyte recognition by autoreactive $T$ cells induced by Theiler's virus infection: Role of viral strains and capsid proteins. J Neuroimmunol $\underline{172}, 85-93$

Tuckermann JP, Kleiman A, McPherson KG, Reichardt HM (2005): Molecular mechanisms of glucocorticoids in the control of inflammation and lymphocyte apoptosis. Crit Rev Clin Lab Sci $\underline{42}, 71-104$

Tuohy VK, Lu Z, Sobel RA, Laursen RA, Lees MB (1989): Identification of an encephalitogenic determinant of myelin proteolipid protein for SJL mice. J Immunol Baltim Md $1950 \underline{142}$, 1523-1527

Usher MG, Duan SZ, Ivaschenko CY, Frieler RA, Berger S, Schütz G, Lumeng CN, Mortensen RM (2010): Myeloid mineralocorticoid receptor controls macrophage polarization and cardiovascular hypertrophy and remodeling in mice. J Clin Invest 120, 3350-3364

Varga G, Ehrchen J, Tsianakas A, Tenbrock K, Rattenholl A, Seeliger S, Mack M, Roth J, Sunderkoetter C (2008): Glucocorticoids induce an activated, anti-inflammatory monocyte subset in mice that resembles myeloid-derived suppressor cells. J Leukoc Biol 84, 644-650

Varga G, Ehrchen J, Brockhausen A, Weinhage T, Nippe N, Belz M, Tsianakas A, Ross M, Bettenworth D, Spieker T, et al. (2014): Immune suppression via glucocorticoid-stimulated monocytes: a novel mechanism to cope with inflammation. J Immunol Baltim Md $1950 \underline{193}, 1090-1099$

Viglietta V, Baecher-Allan C, Weiner HL, Hafler DA (2004): Loss of functional suppression by CD4+CD25+ regulatory T cells in patients with multiple sclerosis. J Exp Med 199, 971-979 
Waddell DS, Baehr LM, van den Brandt J, Johnsen SA, Reichardt HM, Furlow JD, Bodine SC (2008): The glucocorticoid receptor and FOXO1 synergistically activate the skeletal muscle atrophyassociated MuRF1 gene. Am J Physiol Endocrinol Metab 295, E785-797

Weber MS, Prod'homme T, Youssef S, Dunn SE, Rundle CD, Lee L, Patarroyo JC, Stüve O, Sobel RA, Steinman L, Zamvil SS (2007): Type II monocytes modulate T cell-mediated central nervous system autoimmune disease. Nat Med $\underline{13}, 935-943$

Weinstein RS (2012): Glucocorticoid-Induced Osteoporosis and Osteonecrosis. Endocrinol Metab Clin North Am $\underline{41}, 595-611$

Weissleder R, Nahrendorf M, Pittet MJ (2014): Imaging macrophages with nanoparticles. Nat Mater $\underline{13}, 125-138$

Wu F, Cao W, Yang Y, Liu A (2010): Extensive infiltration of neutrophils in the acute phase of experimental autoimmune encephalomyelitis in C57BL/6 mice. Histochem Cell Biol $\underline{133}$, 313322

Wüst S, van den Brandt J, Tischner D, Kleiman A, Tuckermann JP, Gold R, Lühder F, Reichardt HM (2008): Peripheral T cells are the therapeutic targets of glucocorticoids in experimental autoimmune encephalomyelitis. J Immunol Baltim Md 1950 180, 8434-8443

Wüst S, Tischner D, John M, Tuckermann JP, Menzfeld C, Hanisch U-K, van den Brandt J, Lühder F, Reichardt HM (2009): Therapeutic and Adverse Effects of a Non-Steroidal Glucocorticoid Receptor Ligand in a Mouse Model of Multiple Sclerosis. PLoS ONE 4 , 12 e8202

Xu L, Kitani A, Fuss I, Strober W (2007): Cutting edge: regulatory T cells induce CD4+CD25-Foxp3- T cells or are self-induced to become Th17 cells in the absence of exogenous TGF-beta. J Immunol Baltim Md 1950 178, 6725-6729

Yuan B, Zhao L, Fu F, Liu Y, Lin C, Wu X, Shen H, Yang Z (2014): A novel nanoparticle containing MOG peptide with BTLA induces $T$ cell tolerance and prevents multiple sclerosis. Mol Immunol $\underline{57}$, 93-99

Zamvil S, Nelson P, Trotter J, Mitchell D, Knobler R, Fritz R, Steinman L (1985): T-cell clones specific for myelin basic protein induce chronic relapsing paralysis and demyelination. Nature $\underline{317}$, 355-358

Zhang G, Zhang L, Duff GW (1997): A negative regulatory region containing a glucocorticosteroid response element (nGRE) in the human interleukin-1beta gene. DNA Cell Biol $\underline{16}, 145-152$

Zhang G-X, Yu S, Gran B, Li J, Siglienti I, Chen X, Calida D, Ventura E, Kamoun M, Rostami A (2003): Role of IL-12 receptor beta 1 in regulation of T cell response by APC in experimental autoimmune encephalomyelitis. J Immunol Baltim Md 1950 171, 4485-4492

Zhang X, Koldzic DN, Izikson L, Reddy J, Nazareno RF, Sakaguchi S, Kuchroo VK, Weiner HL (2004): IL10 is involved in the suppression of experimental autoimmune encephalomyelitis by CD25+CD4+ regulatory T cells. Int Immunol $\underline{16}, 249-256$

Zhang Z, Zhang Z-Y, Schluesener HJ (2009): Compound A, a plant origin ligand of glucocorticoid receptors, increases regulatory $T$ cells and $M 2$ macrophages to attenuate experimental autoimmune neuritis with reduced side effects. J Immunol Baltim Md $1950 \underline{183}$, 3081-3091 
Zindler E, Zipp F (2010): Neuronal injury in chronic CNS inflammation. Best Pract Res Clin Anaesthesiol 24, 551-562

Zivadinov R, Rudick RA, Masi RD, Nasuelli D, Ukmar M, Pozzi-Mucelli RS, Grop A, Cazzato G, Zorzon M (2001): Effects of IV methylprednisolone on brain atrophy in relapsing-remitting MS. Neurology $\underline{57}, 1239-1247$ 


\section{Acknowledgements}

Many are the people who, in one way or another, contributed to this work.

First, I would like to thank Prof. Dr. Holger Reichardt for placing his trust in me to carry out this interesting project, offering me the opportunity to learn and grow as a scientist. I would also like to acknowledge his guidance and organizing skills in both scientific and bureaucratic matter, and his support in the ups and downs during these years. The door of his office was always open for counseling and interactive discussions, and I really appreciated it.

Many thanks to my second supervisor, PD Dr. Fred Lühder, whom I cannot officially name 'Doktorvater', but he earned at least the degree of 'Doktoronkel'. I thank him for his close supervision, his assistance in practical laboratory matters and for the refreshing scientific discussions. I am also thankful for his guidance through the lights and shadows of the German language.

I would also like to thank Prof. Dr. Hanisch and Prof. Dr. Walter as members of the Thesis Committee, and the rest of the members of the Examination Board, Prof. Dr. StadelmannNessler, Prof. Dr. mult. Meyer, and Prof. Dr. Jarry. I am also thankful to the SFB TRR43 for offering the opportunity to participate in conferences and talks that gave me a broader perspective about the neuroimmunology field.

I am personally really grateful to Dr. Erik Meskauskas, coordinator of the MolMed program, for his help with the bureaucracy and his friendliness. Thanks also to the new coordinator of the PhD students, Ms. Wolfram; to our secretaries in the Immunology Department, Anika Schindler and Ingrid Teuteberg; and to Cathy Ludwig from the Institute for Multiple Sclerosis Research (IMSF).

Not to forget our collaborators in the Institute for Technology in Karlsruhe, Prof. Dr. Feldmann and Dr. Heck, and specially our colleagues in the IMSF here in Göttingen, where I conducted a big part of my experiments. Thanks to Tanja, Anne and Judith for their help in 
many experiments and for their friendliness. Also to César, for making my visits to the IMSF more amusing. To the rest of the PhDs, postdocs and PIs, who were always helpful and contributed to my work with nice feedback. And of course thanks to the animal care takers and the technicians, particularly to Martina Weig, who taught me all about histology and was always willing to help whenever something went wrong.

Next, I would like to thank my colleagues from the Institute for Immunology. Thanks for the many nice moments lived together, both scientific and extra-professional. I am deeply grateful to our technicians, Julian Koch and Amina Bassibas, without them this work would have not been possible. To Amina, thanks for being not only a great technical assistant, but my personal confident and a good friend as well. To all the colleagues that in these years worked with me in the Reichardt's group: Jennifer, Xiao, Kai, Nils, Anna, Ann-Kathrin, Eric, Milena, Katharina, Henrike, Sybille, Nicky, Laura, Garrit and Tina. To the master students that were under my supervision: Sarah, Vicky, Natalie and Elena, it was great to work with you girls! Thanks to Carina, for being always by my side since we started together in the lab. Also to Julius, always friendly and helpful inside and outside the lab. To both of them and Annemarie, extra thanks for the proofreading of this work.

I also want to express my gratitude to all the friends that gave me their support throughout these years. To the ones I made in Göttingen, thank you for making me feel at home in this town.

Finally, an important part of these acknowledgements goes to my family. To my brothers Ignacio and Alberto, and especially to my parents, Toyi and Justo, to whom I dedicate this thesis. Thank you for the education you gave me, which brought me this far, and for being always there, come what may. Foremost, I want to thank you Pedro for always encouraging me to give my best, for your caring support and for making every day colorful and fun. 


\section{Curriculum Vitae}

$\begin{array}{ll}\text { Name } & \text { Elena Victoria Montes Cobos } \\ \text { Date of birth } & 06.02 .1988 \\ \text { Nationality } & \text { Spanish } \\ \text { Contact } & \text { Institute for Cellular and Molecular Immunology } \\ & \text { Humboldtallee 34, 37075 Göttingen (Germany) } \\ & \text { Telephone: +49 (0551) 39-5816 } \\ & \text { E-mail: elena.montes.cobos@gmail.com }\end{array}$

\section{Education}

09/2012 - present PhD candidate at the Institute for Cellular and Molecular Immunology - UMG, Göttingen

09/2011 - 09/2012 Master of Science in Molecular Medicine - UMG, Göttingen

09/2006 - 07/2011 Licentiate in Biotechnology - University of Salamanca, Spain

09/2004 - 07/2006 Secondary School - I.E.S. Valle del Jerte, Plasencia, Spain

09/1992 - 07/2004 Primary School - Colegio San Calixto, Plasencia, Spain

\section{Publications}

Montes-Cobos E, Li X, Fischer HJ, Sasse A, Kügler S, Didié M, Toischer K, Fassnacht M, Dressel R, Reichardt HM (2015): Inducible Knock-Down of the Mineralocorticoid Receptor in Mice Disturbs Regulation of the Renin-Angiotensin-Aldosterone System and Attenuates Heart Failure Induced by Pressure Overload. PloS One 10, e0143954

Theiss-Suennemann J, Jörß K, Messmann JJ, Reichardt SD, Montes-Cobos E, Lühder F, Tuckermann JP, AWolff H, Dressel R, Gröne H-J, et al. (2015): Glucocorticoids attenuate acute graft-versus-host disease by suppressing the cytotoxic capacity of CD8 (+) T cells. J Pathol $\underline{235}, 646-655$ 